\title{
On the Roles of Drosophila Notch Signaling and Atonal Autoregulation in the Specification of R8 Photoreceptors
}

Adam T. Majot

Follow this and additional works at: https://researchrepository.wvu.edu/etd

\section{Recommended Citation}

Majot, Adam T., "On the Roles of Drosophila Notch Signaling and Atonal Autoregulation in the Specification of R8 Photoreceptors" (2015). Graduate Theses, Dissertations, and Problem Reports. 6144. https://researchrepository.wvu.edu/etd/6144

This Dissertation is protected by copyright and/or related rights. It has been brought to you by the The Research Repository @ WVU with permission from the rights-holder(s). You are free to use this Dissertation in any way that is permitted by the copyright and related rights legislation that applies to your use. For other uses you must obtain permission from the rights-holder(s) directly, unless additional rights are indicated by a Creative Commons license in the record and/ or on the work itself. This Dissertation has been accepted for inclusion in WVU Graduate Theses, Dissertations, and Problem Reports collection by an authorized administrator of The Research Repository @ WVU.

For more information, please contact researchrepository@mail.wvu.edu. 


\title{
On the roles of Drosophila Notch signaling and atonal autoregulation in the specification of $\mathrm{R} 8$ photoreceptors.
}

\author{
Adam T. Majot \\ Dissertation submitted to the \\ Eberly College of Arts and Sciences \\ at West Virginia University \\ in partial fulfillment of the requirements \\ for the degree of \\ Doctor of Philosophy \\ In \\ Biological Sciences
Dr. Ashok Bidwai, Ph.D., Chair
Dr. Clifton Bishop, Ph.D.
Dr. Philip Keeting, Ph.D.
Dr. Peter Mathers, Ph.D.
Dr. Shuo Wei, Ph.D.
Department of Biology
Morgantown, West Virginia
2015

Keywords: Drosophila, Notch, Atonal, Anterior open, E(spl), Dpp

Copyright 2015 Adam Majot 


\title{
ABSTRACT \\ On the roles of Drosophila Notch signaling and atonal autoregulation \\ in the specification of $\mathrm{R} 8$ photoreceptors.
}

\author{
Adam T. Majot
}

Drosophila retinogenesis has long served as a paradigm of tissue patterning. Notch, an anciently conserved signaling pathway, is critical to both the formation of and proper spacing of founding R8 photoreceptors. Notch is intimately interwoven into the activity of Atonal (Ato), a proneural basic helix-loop-helix (bHLH) that is required for R8 specification. Ato is expressed through dual enhancers: ato-3' and 5'-ato. ato-3' is activated in response to Notch-independent signaling cues whereas $5^{\prime}$-ato exhibits complex dependence on Notch signaling. First, Notch is required for 5'-ato activation, eliciting formation of Ato intermediate groups (IGs, evenly spaced clusters of 10-20 cells). Subsequently, Notch is required to repress ato, to resolve its expression from IGs to isolated R8s. This is accomplished through the induction of $\mathrm{E}(\mathrm{spl}) \mathrm{bHLH}$ repressors to disrupt proneural function. The former process is independent of the Notch pathway transcription factor Suppressor of Hairless $(\mathrm{SuH})$ and the latter requires $\mathrm{Su}(\mathrm{H})$. Current views regarding this process suggest that either 1 ) the $\mathrm{Su}(\mathrm{H})$-dependent response may require a greater threshold of Notch signal activity before engaging, or 2) $\mathrm{E}(\mathrm{spl})$ repressors are initially expressed but lack proper posttranslational activation to repress ato. The work of Chapter 2 refutes both scenarios. We demonstrate that $\mathrm{E}(\mathrm{spl}) \mathrm{bHLHs}$ are expressed prior to the perceived activation of 5'-ato. $\mathrm{E}(\mathrm{spl})$ repressors are active at this time, as their removal elicits precocious de-repression of $5^{\prime}$-ato. Thus, both the $\mathrm{Su}(\mathrm{H})$-independent and -dependent processes initiate simultaneously. These findings suggest that ato-3' activity is crucial during IG formation. Analysis of the hypermorphic allele $E(s p l)^{D}$ delineates a role for posttranslational activation of E(spl)M8 during the repressive phase of Ato patterning. The work of Chapter 3 further probes the importance of ato-3' in the formation of IGs. Anterior open (Aop) has previously been characterized as an effector of MAPK and is an ortholog of tumor suppressor Tel/ETV6. Aop coexpresses with Ato only during early IG formation, prior to the onset of autoregulation via 5'-ato. We demonstrate that Aop is required cellautonomously during early IG formation to promote continued expression from ato-3'. Furthermore, Aop is induced by Notch activity via $\mathrm{Su}(\mathrm{H})$, indicating that $\mathrm{Su}(\mathrm{H})$ co-opts Aop to instill a delay in Notch-mediated repression. The work of Chapter 4 assesses the role of ato in a broader context. The region of the developing eye that expresses Ato is referred to as the morphogenetic furrow (MF). The MF relies upon sophisticated signaling between Notch, Hedgehog (Hh) and Decapentaplegic (Dpp). Our work indicates that, in addition to Ato's role in facilitating neurogenesis, it is proactive in the production of neural progenitors through its elicitation of Dpp, as assessed by a lacZ reporter. In total, these studies emphasize the complexity of the relationship between Notch and Ato and suggest interesting new studies into possible nodes of signaling crosstalk with Notch. 


\section{ACKNOWLEDGEMENT}

I came into this whole process terribly green, only knowing that I enjoy research and the big pay-off from occasionally "guessing" correctly. Ashok helped, as much as any soul can help me, to focus that passion toward a tangible goal (this here tome). My strongest early memory of life in the Bidwai lab is of a late afternoon lab meeting in which Ashok used the last several minutes to propose a simple idea about Notch signaling and ongoing mysteries of fly eye development. He proposed that biphasic Notch, as outlined in the preceding Abstract, is but a dream. In place of this, Ashok promoted the likelihood that E(spl) was translated early but activated later. As powerful as this idea became for me, I had no means of exploring the idea at the time. Later, in following up on a request of Ashok's, I had obtained aop mutant flies. Out of curiosity, I stained them $\alpha$-Ato. The outcome (Chapter 3, Fig. 2) had finally provided a means to cut to the core of biphasic Notch and see what really made the process work. What this long-winded historical perspective is getting at is that above my deep gratitude for being offered a position in Ashok's lab and for the many wonderful get togethers hosted at the Bidwai house, I am profoundly indebted to Ashok for having taught me how to ask questions. It has been a most sincere joy learning from Ashok how to question the authority of what is printed.

I would be remiss to leave out my committee, Drs. Clif Bishop, Phil Keeting, Pete Mathers and Shuo Wei. Each has been most helpful, either in times of personal need or professional, and often times both. I also look back fondly of the days when both Luke and Mohna were in lab.

Thank you to those in the department who facilitated this work and the work of all graduate students. The tireless efforts of Pat, Wendy, Mickey and Judy always helped to make student life a bit easier than it ever needed to be.

Lastly, I would like to thank my family, including my parents and in-laws. Most importantly, I am grateful for Kylie, my wonderful wife, for bearing with me for these long past twelve months. She and our beautiful daughters, Violet and Clementine are my entire reason for being. 
TABLE OF CONTENTS

\begin{tabular}{|c|c|}
\hline Section & Page(s) \\
\hline Abstract & ii \\
\hline Acknowledgement & iii \\
\hline Table of Contents & iv \\
\hline List of Figures & $\mathrm{v}$ \\
\hline List of Reagents & vi \\
\hline Chapter 1: Introduction & $1-42$ \\
\hline Development, an overview & $1-3$ \\
\hline Notch signaling & $3-9$ \\
\hline Drosophila EGF Receptor signaling & $9-12$ \\
\hline The developing eye & $13-15$ \\
\hline The morphogenetic furrow & $15-19$ \\
\hline Ato & $19-25$ \\
\hline E(spl) & $25-34$ \\
\hline Aop & $34-36$ \\
\hline Scope of Dissertation & 36 \\
\hline Chapter 2: An analysis of ... E(spl)D & 43-68 \\
\hline Abstract & 43 \\
\hline Introduction & $44-46$ \\
\hline Results & 47-54 \\
\hline Discussion & $54-59$ \\
\hline Materials and Methods, Acknowledgements & $59-61$ \\
\hline Chapter 3: Drosophila aop delays E(spl)-mediated repression... & $69-106$ \\
\hline Abstract & 69 \\
\hline Introduction & 70-73 \\
\hline Results & $73-87$ \\
\hline Discussion & $87-92$ \\
\hline Materials and Methods, Acknowledgements & $92-95$ \\
\hline Chapter 4: Ato facilitates anteroposterior transition... & $107-135$ \\
\hline Abstract & 107 \\
\hline Introduction & $108-111$ \\
\hline Results & $111-118$ \\
\hline Discussion & $118-121$ \\
\hline Materials and Methods, Acknowledgements & $122-124$ \\
\hline Future Perspectives & $132-133$ \\
\hline References & $134-154$ \\
\hline
\end{tabular}




\section{LIST OF FIGURES}

\begin{tabular}{|c|c|}
\hline Figures & Page \\
\hline \multicolumn{2}{|l|}{ Chapter 1: Introduction } \\
\hline Fig. 1. Notch is a juextacrine signaling pathway & 37 \\
\hline Fig. 2. Notch is utilized for three major developmental processes & 38 \\
\hline Fig. 3. DER is an autocrine and paracrine RTK signaling pathway & 39 \\
\hline Fig. 4. Compartmentalization of the developing retina & 40 \\
\hline Fig. 5. Ato is dynamically expressed within the MF & 41 \\
\hline Fig. 6. E(spl)bHLHs repress proneural function & 42 \\
\hline \multicolumn{2}{|l|}{ Chapter 2: An analysis of transient hypermorphic activity of $E(s p l) D . .}$. \\
\hline Fig. 1. E(spl) coexpresses with Ato during early IG formation & 62 \\
\hline Fig. 2. E(spl) represses Ato autoregulation & 63 \\
\hline Fig. 3. Dosage contributions to the $N s p l ; E(s p l) D$ interaction & 64 \\
\hline Fig. 4. roe interacts with Ato-sensitized mutants & 65 \\
\hline Fig. 5. roe interacts with $E(s p l) D$ & 66 \\
\hline Fig. 6. $E(s p l) D$ perturbs $\mathrm{R} 8$ paterning with reduced proneural dosage & 67 \\
\hline Fig. 7. A mechanism for $\mathrm{E}(\mathrm{spl})$ repression of ato & 68 \\
\hline \multicolumn{2}{|l|}{ Chapter 3: Drosophila aop delays E(spl)-mediated repression of ato } \\
\hline Fig. 1. Apical constriction is enhanced in neurogenic mutants & 96 \\
\hline Fig. 2. Aop is cell-autonomously required for Ato IGs & 97 \\
\hline Fig. 3. Aop and Ato coexpress during early IG formation & 98 \\
\hline Fig. 4. aop loss-of-function perturbs both ato enhancers & 99 \\
\hline Fig. 5. aop mutation does not misregulate Ci processing and $d p p$ & 100 \\
\hline Fig. 6. Aop requires Notch signaling for its expression in eye discs & 101 \\
\hline Fig. 7. Aop represses ro in the MF & 102 \\
\hline Fig. 8. Aop does not regulate roe or Bar reporters & 103 \\
\hline Fig. 9. Aop does not regulate $E(s p l)$ & 104 \\
\hline Fig. 10. aop loss-of-function sensitizes the eye to $\mathrm{E}(\mathrm{spl})$ & 105 \\
\hline Fig. 11. Aop indirectly opposes $\mathrm{E}(\mathrm{spl})$ repression of Ato... & 106 \\
\hline \multicolumn{2}{|l|}{ Chapter 4: Ato facilitates anteroposterior transition... } \\
\hline Fig. 1. Ato precedes induction of $d p p-l a c Z$ & 125 \\
\hline Fig. 2. Notch does not appear to directly regulate ato-3' & 126 \\
\hline Fig. 3. ato mutants fail to activate $d p p$-lacZ & 127 \\
\hline Fig. 4. Notch signaling prevents prolonged Ciact accumulation & 128 \\
\hline Fig. 5. Ato and Da negatively regulate Ey in the MF & 129 \\
\hline Fig. 6. MF progression is slowed through proneural mutants & 130 \\
\hline Fig. 7. Ato confers both anterior and posterior compartmental identity... & 131 \\
\hline
\end{tabular}




\section{LIST OF REAGENTS}

\begin{tabular}{|c|c|c|c|}
\hline Genotype & Source & Citation & Nature \\
\hline w1118 & Lab stock & $\mathrm{n} / \mathrm{a}$ & WT \\
\hline $\begin{array}{l}\text { frt82B Df(3R)E(spl)b32.2P\{ry+t7.2 } \\
\text { gro\} }\end{array}$ & BDSC 52011 & Heitzler et al., 1996 & deletion of entire $\mathrm{E}(\mathrm{spl})$ locus, rescue of gro \\
\hline frt82B E(spl)D & $\begin{array}{l}\text { Lab stock } \\
\text { Lab }\end{array}$ & Oellers, 1993 & encodes $\mathrm{M}^{*}$ and removes insulator between $\mathrm{bH} L \mathrm{Hs}$ and gro \\
\hline Nspl & stock/BDSC & Li et al., 2003 & encodes 1578T; promotes altered glycosylation \\
\hline Df(3R)roern16 & BDSC 7407 & St. Pierre et al., 2002 & removes entire roe coding region \\
\hline Df(3R)roern20 & BDSC 7409 & St. Pierre et al., 2002 & removes enitre $\mathrm{rn}$ locus \\
\hline DEREIp & BDSC 8484 & Lesokhin et al., 1999 & encodes A877T; enhances sensitivity to receptor activation \\
\hline $\mathrm{T}(2 ; 3) \operatorname{RoD} /+;+$ & Justin Kumar & Chanut et al., 2000 & alters upstream enhancer, molecular lesion uncclear \\
\hline hH10 GAL4 & Lab stock & Ellis et al., 1994 & GawB insertion in hairy locus, produces ectopic GAL4 \\
\hline UAS-mDelta & BDSC 26677 & de Celis et al., 1996 & EcoRI-Xhol fragment of mDelta cDNA ligated into pUAST \\
\hline sensE2 & $\begin{array}{l}\text { BDSC } 5311 \\
\text { Graeme }\end{array}$ & Wang et al., 2010 & missense mutation at (3L) 13393009 \\
\hline frt82B ato1 & Mardon & Jarman et al., 1994 & encodes N261I, K253N, A25T; ablates DNA binding \\
\hline aop1 frt40A & Justin Kumar & $\begin{array}{l}\text { Caviglia and Luschnig, } 2013 \\
\text { Morel and Schweisguth, }\end{array}$ & nonsense mutation G952A, ablates DNA binding \\
\hline $\mathrm{Su}(\mathrm{H})^{\wedge} 47 \mathrm{frt} 40 \mathrm{~A} P\{(2) \mathrm{Bg} 35+\}$ & Nick Baker & 2000 & deletion of $\mathrm{Su}(\mathrm{H})$ and $\mathrm{I}(2) 35 \mathrm{Bg}$ transcriptional starts \\
\hline aopyan1 frt40A & BDSC 8780 & Lai and Rubin, 1992 & removal of cyto. 22A2-3 thru 22D5-E1 \\
\hline $\mathrm{P}\{\mathrm{W}+\mathrm{mc}$ ato5' $\mathrm{F}: 9.3\}$ & Yuh Nung Jan & Sun et al., 1998 & 9.3kb of adjacent $5^{\prime}$ ato enhancer ligated into pCaSpeR-Beta-gal \\
\hline $\mathrm{P}\{\mathrm{w}+\mathrm{mC}$ ato3' $\mathrm{F}: 5.8\}$ & Yuh Nung Jan & Sun et al., 1998 & $\begin{array}{l}1.1 \mathrm{~kb} \text { of adjacent } 5^{\prime} \text { ato enhancer and } 5.8 \mathrm{~kb} \text { of adjacent } 3^{\prime} \text { ato } \\
\text { enhancer }\end{array}$ \\
\hline frt82B pnt^${ }^{\wedge} 88$ & Justin Kumar & Scholz et al., 1993 & removal of entire pnt-P1 and $3^{\prime}$ exon of pnt-P2 \\
\hline N55e11 frt19A & BDSC 28813 & Kidd et al., 1986 & 3.5kb insertion in $5^{\prime}$ coding region, premature nonsense mutation \\
\hline frt82B rox63 & BDSC 6335 & Kimmel et al., 1990 & deletion in 5 ' coding region, frameshift after $30 \mathrm{aa}$ \\
\hline Mi\{MIC\}rn[MI07760] & $\begin{array}{l}\text { BDSC } 44338 \\
\text { Tetsuya }\end{array}$ & Venken et al., 2011 & $\operatorname{Mi}\{\mathrm{MIC}\}$ insertion in rn locus \\
\hline $\begin{array}{l}\text { BarP058-lacZ } \\
\text { P\{w+mc dpp-GAL4.blk }\}\end{array}$ & $\begin{array}{l}\text { Kojima } \\
\text { BDSC } 1553\end{array}$ & $\begin{array}{l}\text { Sato et al., } 1999 \\
\text { Staehling-Hampton et al., } \\
1994\end{array}$ & $\begin{array}{l}\text { P\{lacW }\} \text { insertion in Bar locus } \\
4 \mathrm{~kb} \text { of } 3 \text { ' dpp "blk" enhancer ligated into nondescript } P\{w+G A L 4\} \\
\text { vector }\end{array}$ \\
\hline UAS-eGFPnls & BDSC 4775 & Robertson et al., 2003 & \\
\hline$P\{w+m c$ UAS-aopAct $\}$ & BDSC 5789 & Rebay and Rubin, 1995 & eight putative phosphacceptor Ser residues mutagenized to Ala \\
\hline frt82B DIRevF10 SerRX82 & BDSC 6300 & Ligoxygakis et al., 1998 & imprecise P-element excision \\
\hline emcAP6 frt80B & BDSC 36544 & Ellis, 1994 & 400bp deletion, mutants lack antigenicity in immunoassays \\
\hline P\{BS3.0\} (dpp-lacZ) & BDSC 5527 & Blackman et al., 1991 & $12 \mathrm{~kb}$ region of dpp "disk" enhancer ligated into plasmid HZ5OPL \\
\hline P\{BS3.0\} (dpp-lacZ) & BDSC 5528 & Blackman et al., 1991 & $12 \mathrm{~kb}$ region of dpp "disk" enhancer ligated into plasmid HZ5OPL \\
\hline da10 frt40A & BDSC 5531 & Caudy et al., 1988 & 4kb deletion, mutants lack antigenicity in immunoassays \\
\hline Antibody & Source & Citation & \\
\hline $\mathrm{Rb}$ anti-Ato & Yuh Nung Jan & Jarman et al., 1994 & \\
\hline Ms anti-E(spl) mAb323 & Sarah Bray & Jennings et al., 1994 & \\
\hline Gp anti-Sens & Hugo Bellen & Nolo et al., 2000 & \\
\hline
\end{tabular}




\section{Chapter 1: Introduction}

\section{Development}

Metazoan bodies are built through repeated mitosis and intercellular signaling events. Animal bodies, despite their remarkable diversity, are largely constructed using the five conserved signals of Hedgehog (Hh), Decapentaplegic (Dpp), Notch, Wingless (Wg) and the receptor tyrosine kinase family (RTK). Despite the use of only five major signaling pathways, the specification of a multitude of cell types is possible through reiterative and combinatorial usage of these pathways. The study of development and intercellular signaling is enabled by past advances in genetics, a field that is generally considered to have begun in earnest within the laboratory of Thomas Hunt Morgan, in the early $20^{\text {th }}$ century at Columbia University (Kohler 1994). Initial analyses, though not originally intended toward such ends, described discrete, heritable mutations. Many of them were culturable, hence proving to be amenable to trans-generational maintenance. Such work in cataloguing mutations begat the precipitation of the concept of the chromosome as the organizational superstructure of genes. In the process of this work, mutations that disrupted the aforementioned signaling pathways were isolated, and such conserved signaling pathways and their orthologs bear the name of the original Drosophila mutants. To this day, Drosophila has maintained a prominent role in the study of genetics and intercellular signaling, owing partly to its historical position, but moreover to the species' continued facility in the discovery of principles that govern the construction of animal bodies and the reliance of such processes on genes and their products.

\section{Intercellular signaling coordinates development.}

Cell fate determination requires the interaction of transcription factor networks with signaling effectors. A general problem in development is that a small number of pathways are largely responsible for the organization of the developmental program, and such pathways are utilized in similar context across many different tissues. For example, both Drosophila eye and wing 
development exhibit simultaneous use of Hedgehog (Hh), Decapentaplegic (Dpp) and Notch signaling, but the combination of signals elicits a very different outcome in each respective tissue. Specialization of cells is most simply accounted for in a two-input model of cell fate determination, wherein tissues are first provided a cell-intrinsic expression profile that can later be modulated by extrinsic signals (Yuh et al., 1998; Ghazi and VijayRaghavan 2000). Cell-intrinsic expression patterns are elicited through the activity of a gene or system of genes that uniquely characterize each developing tissue. Homeotic genes, or master control genes, serve such a role throughout Animalia, designating body regions during early embryogenesis and directing the expression of genes that induce the development of tissues specific to individual body regions (Lewis 1992). Extrinsic cues, from signaling pathways, can then act on particular subsets of cells within a given tissue, facilitating differentiation of a variety of cell types.

A simple example of such regulation can be found in the analysis of Drosophila hh, for which a single enhancer module governs eye-specific expression of Hh. This enhancer requires two inputs, SIX family homeobox Sine oculis (So) and the ETS transactivator Pointed (Pnt) (Rogers et al. 2005; Pauli et al. 2005). So expression in the eye is contingent upon proper activation of a system of genes termed the retinal determination gene network (RDGN). Pnt becomes activated as a terminal effector of Drosophila epidermal growth factor receptor (DER) signaling. Thus, in this circumstance So provides a cell-intrinsic specification as eye anlage and Pnt permits the extrinsic signal activation of the target gene $h h$. As implicated in this model, signaling is of premier importance in cells' progressive transition from having broad potential to the acquisition of terminal fates.

The following analyses aim to better define the role of the anciently conserved Notch signaling pathway during a critical stage of Drosophila eye development. To obtain a tissue-specific understanding of Notch requires that we also understand the proneural bHLH Atonal (Ato), the primary cell-intrinsic factor with which Notch signaling acts in the developing retina. Given that 
the roles of Ato and Notch are intricately intertwined with the activation of the DER (an RTK) signaling pathway, we provide a detailed characterization of both Notch and DER signaling, highlighting canonical pathways of information transmission, and augmented with descriptions of each pathway's roles in development. We then proceed with an explanation of Drosophila retinogenesis with an emphasis on the role of Ato, its known effectors, and what remains yet to be understood.

\section{Notch signaling overview.}

Notch signaling is a juxtacrine pathway that is utilized in both development and homeostasis. Thus, aberrant signaling can elicit birth defects, age-related disease and cancer (as reviewed in Andersson and Lendahl, 2014). In brief, the juxtacrine pathway requires close proximity between signal-sending and signal-receiving cells, as both ligand and receptor are transmembrane proteins. Ligand-receptor interaction stimulates ligand endocytosis on the ligandbearing cell, triggering two proteolytic events that ultimately free an intracellular portion of the Notch receptor into the cytosol of the signal-receiving cell. The intracellular fragment of Notch subsequently translocates to the nucleus where it exerts its effects on gene expression by converting a transcriptional repressor complex into a transactivator (Fig. 1). Mechanistically, Notch is best detailed for its developmental roles in lateral inhibition, assymetric inheritance, and boundary formation/maintenance (Fig. 2).

\section{Architecture of the Notch receptor.}

Notch is a large (300 kDa in Drosophila), single-pass transmembrane glycoprotein that ranges in size across species and variant forms, though the essential functional domains are conserved. From N- to C-terminus, the receptor features multiple epidermal growth factor (EGF) repeats (36 in Drosophila), three Lin12/Notch repeats (LNRs), a heterodimerization domain (HD), a transmembrane domain, an RBP-J associated module (RAM) domain, several Ankyrin repeat domains (ANK), flanked either N-, C-terminally, or at both ends by nuclear localization sequence 
(NLS) motifs, a transactivational domain and a proline, glutamate, serine, threonine (PEST) domain

(Fig. 1A). The single Notch isoform in the Drosophila genome is advantageous in signaling studies, eliminating the possibility of redundancy, as can be observed in mammals, whose genomes generally encode four Notch receptors that are employed in different combinations throughout development.

Receptor maturation requires extensive processing - proteolytic cleavage and subsequent heterodimerization, glycosylation, coordination of $\mathrm{Ca}^{2+}, \mathrm{Zn}^{2+}$ and the formation of disulfide bridges. In the golgi, a furin-like convertase cleaves the nascent polypeptide at site S1 within the the HD, separating the receptor into two subunits that heterodimerize through contacts between the two tracts of HD (Fig. 1; Lake et al., 2009). The 0-fucosyltransferase 0-fut1 promotes the addition of fucose to Ser or Thr residues of various EGF repeats on the Notch extracellular domain (Okajima and Irvine, 2002). O-fucosylation by 0 -fut1 promotes ligand binding and Notch signaling, and depletion of 0 -fut1 results in phenotypic defects that are characteristic of Notch loss of function (Nlof; Sasamura et al., 2003). Aside from its enzymatic activity, O-fut1 assists in the proper folding of Notch, as was demonstrated in the absence of O-fut1 catalytic activity (Okajima et al., 2005; Stahl et al., 2008). The addition of O-fucose provides support for further O-glycosylation events by the enzymes Rumi and Fringe (Fng). Rumi is a conserved, soluble, ER protein O-glucosyltransferase that modifies Notch, and in mammals, has been shown to exhibit dual specificity, also catalyzing the transfer of Xylose to EGF repeat 16 of NOTCH1 (Acar et al., 2008; Takeuchi et al., 2011). Mutations to rumi result in the accumulation of both intracellular and cortical Notch and elicit temperaturesensitive phenotypic defects that are suggestive of Nlof, consistent with a proposed role for rumi in the folding and trafficking of Notch. O-fut1 mutants also display decreased Notch endocytosis and turnover, further implicating sophisticated regulation of receptor structure and function through glycosylation and interaction with glycosyltransferases (Sasamura et al., 2007). Interestingly, 
cortical Notch in $O$-fut1 and rumi mutants does not display decreased ability to bind ligand, but only reduced ability to initiate signaling.

\section{DSL ligands and Notch signaling mechanism.}

Canonical Notch ligands are of the DSL (Delta/Serrate/LAG-2) family and are similar to the receptor in that they are single-pass transmembrane glycoproteins that they are comprised of multiple EGF-like repeats in their extracellular domains (Fig. 1A). There exist two classes of ligand in both Drosophila and Mammalia, listed respectively: (1) Delta (Dl) or Delta-like (Dll) and (2) Serrate (Ser) or Jagged (Jag). Structurally, all Notch ligands have an N-terminal Module at the Nterminus of Notch ligands (MNNL) domain and DSL domain that cooperatively facilitate trans interaction with the Notch receptor (Fig.1A; Parks et al., 2006; Shimizu et al., 1999). Following the DSL domain is a variable number of EGF repeats, 6-8 in Dl and Dll and 15-16 in Ser and Jag (Fig. 1A). As such, Notch ligands are subject to variable modification by glycosylation, and are likely to have similar influence on folding and trafficking as observed with the Notch receptor, and to modulate interactivity with Notch (Panin et al., 2002). Ser/Jag ligands can be further differentiated from $\mathrm{Dl} / \mathrm{Dll}$ by their additional presence of a cysteine rich domain that is located between the EGF repeats and the transmembrane domain (Fig. 1A).

Notch signaling is initiated when a neighboring cell presents a mature Notch receptor, which suitably interacts with available ligand. The ligand-receptor pair stably interact to stimulate endocytosis of the ligand-receptor into the ligand-expressing cell (Fig. 1B). Endocytosis is signaled by the monoubiquitylation of the ligand intracellular domain by either E3 ubiquitin ligase Neuralized (Neur) or Mindbomb, and the endocytic adaptor protein Epsin (Fig. 1B; Lai and Rubin, 2001; Yeh et al., 2001; Wang and Struhl, 2004; Wang and Struhl, 2005). Tension on the extracellular domain of Notch elicits structural deformation of the LNR domain, exposing the previously concealed S2 cleavage site to ADAM-type metalloproteases, which then act to free ligand-extracellular Notch from the Notch extracellular truncation (NExT) (Gordon et al. 2007). 
The lift-and-cut, or ectodomain shedding model of Notch activation is supported by crystallographic evidence and the sensitivity of the LNR domain to mutation, as gain-of-function mutants in Drosophila, C. elegans and human Notch orthologs have been attributed to mutation of LNR repeats, which further suggest greater access to S2 by ADAM metalloproteases when LNR repeat structure is perturbed (Lieber et al., 1993; Berry et al., 1997; Greenwald and Seydoux, 1990; Weng et al., 2004; Tien et al., 2008). S2 cleavage is followed by proteolysis at S3 via Presenilin (Psn) $/ \gamma$-secretaseactivity (Fig. 1B; De Strooper et al., 1999; Struhl and Greenwald, 1999; Okochi et al., 2002). It remains unclear whether S3 cleavage is first dependent upon NExT endocytosis, leading to speculation that NExT ubiquitylation may play a role in Notch signal processing or that NExT may require interaction with an endocytic chaperone to promote interaction with $\gamma$-secretase (Fig. 1B). Two potential suitors, Deltex (Dx) and Suppressor of Deltex (Su(Dx)), both E3 Ubiquitin ligases, interact with and ubiquitylate the intracellular domain of Notch, but fail to sufficiently fill such a role. Dx promotes Notch signaling, but appears to operate through a noncanonical signaling axis; $\mathrm{Su}(\mathrm{Dx})$ promotes NExT endocytosis, but diminishes rather than promotes Notch signaling (Matsuno et al., 1995; Ramain et al., 2001; Cornell et al., 1999). Regardless of the particular mechanism, S3 cleavage results in the freeing of the Notch intracellular domain (NIcD), which then translocates to the nucleus (Fig. 1B).

Prior to signaling, Notch target genes are arrested in a state of transcriptional silence through the binding of $\mathrm{Su}(\mathrm{H})$ and its associated repressor complex that includes Hairless, which coordinates the co-repressors Groucho (Gro) and C-terminal binding protein (Fig. 1B; Morel et al, 2001; Barolo et al., 2002). NIcD interacts with Su(H) through its RAM domain, displacing corepressors from the complex (Fig. 1B). Co-activator Mastermind (Mam) is then recruited, which interacts with NIcD through its ANK repeats, converting $\mathrm{Su}(\mathrm{H})$ into a potent transactivator (Fig. 1B; Petcherski and Kimble, 2000; Smoller et al., 1990; Schuldt and Brand, 1999). NIcD is destabilized through the presence of the PEST motif, which constitutes a phosphorylation-dependent 
degradation signal (phosphodegron), targeting NIcD by the E3-ubiquitin ligase Archipelago/Fbxw7 to the proteasome (Oberg et al., 2001).

\section{0-glycosylation by Fringe modulates Notch functionality.}

Individual EGF repeats of extracellular Notch regulate the signaling pathway through effects on receptor-ligand interaction and cis-inactivation. EGF repeat 8 is required for interaction with Ser/JAG ligands; whereas EGF repeats 11 and 12 facilitate receptor interaction with Dl/Dll ligands (Rebay et al., 1991; de Celis et al., 1993; Shimizu et al., 1999). As previously intimated, posttranslational modfication has a large role in the regulation of Notch signaling. The 0glycosyltransferase Fng, catalyzes the addition of $\mathrm{N}$-acetylglucosamine to $\mathrm{O}$-fucose moieties on specific EGF repeats of Notch and DSL ligands, serving to potentiate signaling, modulate cisinhibition, and to favor receptor interaction with particular ligands (Fig. 2C; Bruckner et al., 2000; Moloney et al., 2000; Pei and Baker, 2008; Panin et al., 1997). Fng was first described for its role in restricting Wg expression to the DV boundary of the primordial Drosophila wing, a process that relies on precise regulation of Notch signaling (Fig. 2C; Irvine and Wieschaus, 1994). Fng modification of EGF repeat 12 of the Notch receptor serves the dual purpose of promoting transinteraction with Dl while suppressing trans-interaction with Ser - findings that have been recapitulated in mammals, indicating that Fng-Notch represent a broadly conserved model of Notch regulation (Fleming et al., 1997; Shao et al., 2003; Lei et al., 2003; Hicks et al., 2000).

Notch is also regulated by cis-inactivation via non-exclusive intramolecular and intermolecular interactions. Initial studies of Notch cis-inactivation were performed using the Abruptex $(A x)$ mutations, which map to EGF repeats 24, 25, 27 and 29 (Kelley et al., 1987; de Celis and Garcia-Bellido, 1994). $N^{A x}$ flies exhibit excessive Notch signaling, implicating these EGF repeats in some form of negative regulation of Notch. Interaction assays have revealed the possibility that particular regions of extracellular Notch form intramolecular interactions that may regulate ligand binding. The proposed model, a combination of inference based on physical limitations of receptor 
orientation and in vitro binding assays with soluble Notch fragments, suggests that EGF repeats 626 create a looped structure that likely features multiple inter-EGF repeat interactions and promotes cis-inactivation (Xu et al., 2005; Pei and Baker, 2008). In support of this model, despite its vagueness, Fng is unable to suppress Notch signaling in an Abruptex background, nor is the receptor capable of becoming refractory to trans interaction with ligand (de Celis and Bray, 2000), indicating that Fng also negatively regulates receptor-ligand interaction through employment of the Abruptex domain. Other studies indicate that in the golgi, Notch and DSL ligands can form cisinteractions that prevent their proper cortical localization, thus limiting receptor and ligand availability to other other cells, diminishing signaling capacity and further suggesting the use of a ratiometric balance between receptor and ligand in the regulation of Notch signaling (Sakamoto et al., 2002; Fiuza et al., 2010; LeBon et al., 2014).

\section{Notch directs lateral inhibition.}

Notch signaling is required for lateral inhibition, a form of reciprocal inhibitory signaling that is used to limit the number and location of sensory organ precursors (SOPs) in the developing nervous system. Each SOP is the cell from which a bristle sensillum forms, and once specified, the SOP does not appreciably move from its location in the epithelium. Thus, establishment of proper SOP placement is paramount to the proper spacing adult sensory organs. Drosophila nota have 11 pairs of large bristle sensilla, macrochaetae, whose positions are invariant and genetically determined. Additionally, nota are covered in uniformly spaced small bristles that are termed microchaetae. In the classical model of neurogenesis, the SOPs of both type of chaetae are patterned through a similar genetic mechanism, in which proneural proteins of the Achaete-Scute (Asc) family of bHLH transactivators become upregulated within a local group of cells, the proneural cluster (PNC) and activate genes that favor SOP fate (Fig. 2A). However, to restrict SOP fate to only one cell of each PNC, Asc genes also stimulate production of Dl to stimulate Notch signaling in neighboring cells (Fig. 2A). The Notch response activates genes of the Enhancer of split 
complex (E(spl)-C) which encodes bHLH-Orange repressors that function to repress Asc activity and expression (Fig. 2A). (An elaboration on the E(spl) locus follows, p. 25) Thus, cells of each PNC are pitted in competition to express the highest level of Asc in the shortest duration in order to repress neighboring cells from doing the same, thereby permitting the remaining Asc-positive cell to assume SOP fate (Fig. 2A). As expected from this model, $\mathrm{N}^{\text {lof }}$ results in the achievement of multiple SOPs from a single PNC and the formation of multiple adjacent macro- or microchaetae on the adult notum.

\section{DER signaling is required for proper eye development.}

The Drosophila epidermal growth factor receptor (DER) pathway is primarily a mid-range autocrine/paracrine signaling system that is used reiteratively throughout development to induce proliferation and stimulate differentiation. Signal sending cells secrete small protein ligands that stimulate receptor activation (Fig. 3). Upon ligand binding, cells can activate a variety of intracellular responses that all initiate with transphosphorylation of receptor tyrosine kinase domains. In the developing eye, DER is employed repeatedly for the early specification of eye tissue, and later, the differentiation of many cell types (Kumar and Moses 2001a; Kumar and Moses, 2001b; Lesokhin et al., 1999; Yang and Baker, 2001; Yang and Baker, 2003). Aside from eye development, DER signaling is required for diverse purposes such as oogenesis, limb outgrowth, and patterning of the abdomen and wing veins (reviewed in Schweitzer and Shilo, 1997). EGFR is employed similarly in humans, whose genome encodes four paralogous receptors to facilitate a variety of developmental functions. As with the Notch pathway, human EGFR is implicated in a variety of disease states, chief of which is cancer, which stems from EGFR's dual use in both development and homeostasis. The following section focuses solely on the activation of intracellular Ras-MAPK as this is the only DER function that is pertinent to the aspects of eye development that will be addressed in this work (Fig. 3B). 


\section{DER architecture, ligands, and intracellular response.}

DER, like Notch, is a single-pass transmembrane receptor (Fig. 3A). DER functions as a dimer wherein ligand binding stimulates transphosphorylation of Tyr residues of the intracellular domains (Fig. 3B). Each receptor is comprised of an N-terminal extracellular domain that consists of two ligand binding domains (LBDs), with the second LBD flanked by cysteine-rich (CR) dimerization loops (Fig. 3A). The intracellular components are a Src-homology 1 (SH1) Tyr kinase domain that is followed C-terminally by the phosphorylation domain, which houses the Tyr residues that are subject to modification upon receptor activation (Fig. 3A).

In its most simplified form, DER signaling initiates with the binding of one ligand to one receptor. Ligand binding induces conformational change in the receptor that facilitates homodimerization of two receptor-ligand complexes, which, in turn, optimize the positioning of the intracellular domains to facilitate transphosphorylation (Lemmon et al., 1997; Schlessinger, 1988; Zhang et al., 2006). As previously addressed, the human genome encodes multiple EGF receptor isoforms, allowing for greater signaling complexity than that observed in simpler systems that have only one EGF receptor, such as Drosophila. In humans, receptor homodimerization and heterodimerization can regulate ligand specificity and modulate signaling strength and response quality. The lack of permutational complexity with DER has provided many insights into the signaling mechanism used in humans (Schlessinger, 2000). For example, it was first revealed that DER was capable of signaling through receptor asymmetry, a condition in which one receptor molecule binds a ligand and interacts with a second ligand-less receptor to constitute an activated receptor complex (Alvaredo et al., 2010). Though initially thought to be a quirk of Drosophila signaling, crystallographic studies later showed human EGFR signaling to operate through a similar asymmetric principle (Liu et al., 2012). The functional difference in outcomes between symmetric and asymmetric signaling remains unclear. 
Spitz (Spi) is the predominant DER ligand throughout Drosophila development (Fig. 3A; Doroquez and Rebay, 2006). Spi is one of three Drosophila transforming growth factor- $\alpha$ family ligands, along with Keren and Gurken, although of these factors, Spi is utilized exclusively during the pre-differentiation and differentiation phases of eye development that will be discussed in the following text. Spi is first produced as a transmembrane protein with an extracellular EGF domain to facilitate interaction with and activation of DER (Fig. 3A; Rutledge et al., 1992). Through the action of Star (S) and Rhomboid (Rho), Spi matures and is secreted. S chaperones Spi from the ER to Golgi, where Rho, a serine-protease, cleaves Spi, freeing its extracellular domain for subsequent secretion and signal activity (Fig. 3; Lee et al., 2001; Tsruya et al., 2002; Urban et al., 2001).

Downstream of ligand-receptor activation, the phosphorylated intracellular domains of DER recruit Downstream of Receptor Kinase (Drk), a scaffolding protein that bears a Src-homology 2 (SH2) domain, which has high affinity for phospho-Tyr (Fig. 3B). Drk recruits the guanine nucleotide exchange factor Son of Sevenless (Sos), which facilitates the association of the G-protein Ras to the receptor-scaffold complex (Fig. 3B). Sos promotes Ras to exchange GDP for GTP, which allows Ras to recruit Raf, a Ser/Thr kinase, initiating the kinase cascade wherein Raf dually phosphorylates MAP/ERK kinase (MEK), activating the latent Ser/Thr/Tyr kinase activity of MEK (Fig. 3B). In turn, MEK dually phosphorylates MAPK, activating MAPK and allowing its translocation into the nucleus (Fig. 3B). Drosophila have four MAPKs: Rolled (ERK ortholog), Jun Nterminal kinase (Jnk), p38a and p38b, though insufficient testing has been performed to fully associate each gene with a particular set of MAPK-dependent functions.

\section{MAPK directly manipulates gene expression through anterior open and pointed.}

Canonically, MAPK phosphorylates a pair of E-twenty six (ETS) proteins, Anterior open (Aop, ortholog to mammalian Tel1/Etv6) and Pointed (Pnt, ortholog to mammalian Ets1/2), both of which are winged helix-turn-helix transcription factors. Although the two exhibit a limited degree of autonomy, the two bind to many of the same enhancers via ETS DNA-binding domains, 
potentially at the same sequence motifs in some of these cases (Fig. 3; Boisclaire LaChance et al., 2014; Xu et al., 2000). Although both are similarly deployed in many developmental contexts, the two differ in that Aop functions as a repressor and Pnt as a transactivator and that phosphorylation by MAPK exerts different outcomes on each: Aop function is repressed upon phosphorylation whereas Pnt becomes a potent transactivator upon modification (Fig. 3B; Rebay and Rubin 1995; O’Neill et al., 1994). Thus, the functional antagonism exhibited by Aop and Pnt strongly suggests that the two cooperatively regulate a tight transition from low-level to high-level gene expression upon MAPK activation.

\section{Aop represses through SAM-mediated polymerization.}

Aop has a diversity of developmental roles, including photoreceptor specification, embryonic dorsal closure, and providing a block to cell proliferation and differentiation (Tei et al., 1992; Lai and Rubin, 1992; Rebay and Rubin, 1995). Aop, as with Pnt, exhibits preferential binding with the core enhancer motif of G-G-A-A/T and both likely compete for genomic binding substrate (Xu et al., 2000). Repression by Aop is facilitated through the presence of an N-terminal sterile alpha motif (SAM) domain, which permits the formation of multimeric assemblies of Aop (Fig. 3A). As opposed to the chemomodulatory effects of larger chromatin repressor complexes, Aop is proposed to merely bind and prevent expression of euchromatic regions. SAM domains undergo self-assembly, tethered to DNA via Aop ETS domains; such polymers can theoretically consist of an unlimited number of individual Aop molecules. Polymerization-deficient point mutants were generated to further understand whether SAM-SAM interaction drove repression. Aop occupancy of target binding sites was largely unaffected in polymerization-deficient mutant assays, indicating that polymerization does not likely drive Aop binding (Webber et al.; 2013). However, repressive capability was largely ablated in such mutants, demonstrating that the polymerization was critical for Aop-mediated repression. Such data suggest the likely mechanism that polymerization, as opposed to the mere presence of chromatin-bound Aop, facilitates a repositioning of DNA into a 
conformation that is unfavorable for transcriptional activation, potentially through the sequester or segregation of enhancers from their associated promoters.

\section{The developing eye}

External adult Drosophila tissues are derived from cognate anlagen termed imaginal discs, which achieve terminal patterning and differentiation during late larval and pupal development. Imaginal discs are cellular bilayers wherein one layer forms the disc proper and the opposite layer serves as a peripodial, squamous membrane. The distinct advantage of studies within such tissues is that signaling responses within each disc proper is generally limited to those signals that are emitted by other cells of the same disc, and given that the disc proper is a monolayer, signaling is largely restricted to occur in only two-dimensions. The peripodium does not actively participate in fate decisions within the disc, although notable exceptions do exist, as with the use of Hippo signaling in the repression of peripodium-eye disc transformation (Zhang et al. 2011).

The eye, antenna, ocelli, maxillary palps and surrounding head cuticle are derived from a shared tissue, the eye-antennal imaginal disc (Haynie and Bryant, 1986). When referring to the developing eye alone, absent of the antenna and conjoined tissues, we shall henceforth use the term "eye disc," with the understanding that this is just one component of a compound tissue.

Throughout the first and early second instar, the eye disc remains asynchronously mitotic from posterior to anterior. During the mid-second instar, differentiation initiates at the posterior margin of the eye disc, progressing in a dorsoventral column toward the anterior of the tissue. As differentiation proceeds, the eye becomes separated into two compartments, divided by a dorsoventral interface that remains morphologically and genetically distinct from either compartment (Fig. 4A; Ready et al., 1976). The posterior compartment features differentiated cells where ommatidia are progressively constructed, starting with the specification of the R8 photoreceptor and step-wise recruitment of other cell types through inductive signaling (Fig. 4A). Progressive differentiation in the growing posterior compartment continuously encroaches on cells 
of the anterior compartment. The interface between the two compartments is morphologically distinct in that the columnar epithelium of the eye disc becomes constricted at its apical surface, manifesting itself as a physical indentation that stretches dorsoventrally across the tissue with width of 8-10 cells (Wolff and Ready, 1993). This indentation has been termed the morphogenetic furrow, or MF, and its passage toward the anterior is accompanied by the onset of differentiation (Fig. 4A). Following, we will explain the genetic programming that makes each region of the developing eye distinct, and their known points of integration, including an explanation of the signaling mechanisms that drive anterior progression of the MF and the differentiation program. The posterior compartment: sequential differentiation.

The acquisition of terminal retinal fates initiates with the specification of photoreceptors, and the first photoreceptor to be specified is the R8 (Fig. 4B). Isolated R8s emerge from the MF in columns, with uniform DV spacing and proper phasing from the preceeding and proceeding columns. The R8 secretes Spitz (Spi), a DER ligand (Freeman, 1994; Tio et al., 1994; Tio and Moses, 1997). DER, through canonical activation of Pnt, induces the recruitment of the $R 2 / 5$ photoreceptor pair (Fig. 4B; Yang and Baker, 2003). The R8 itself, once differentiated, is disabled from enacting a DER signaling response through its expression of the transcription factor Senseless (Sens), which indirectly prevents the nuclear translocation of MAPK (Frankfort et al., 2001; Frankfort and Mardon, 2004; Pepple et al. 2007). The R2/5, the first pair of retinulae recruited to the R8, contribute to the secretion of Spi and enable the recruitment of the R3/4 photoreceptor pair (Fig. 4B). Cells of uncommitted fate leave their G1 arrest and enter into a single round of mitosis, termed the second mitotic wave, which produces a sufficient number of cells for continued recruitment to nascent ommatidia (Cooper and Bray, 2000; Wolff and Ready, 1991; de Nooij and Hariharan, 1995). Next, combinatorial Notch and DER signaling are used to specify the R1/6/7 photoreceptors and two cones from a five-cell equivalence group (Fig. 4B; Tomlinson et al., 2011). 
Ommatidial construction continues into the pupal stage of development with the recruitment of the polar cones, pigment cells, and interommatidial bristles (Wolff and Ready, 1993).

\section{The anterior compartment: attainment of proneural competency.}

The anterior compartment is characterized by the progressive induction of genes of the conserved transcription factor network termed the retinal determination gene network (RDGN; Bessa et al., 2002). The master regulator protein Eyeless, Ey, is expressed throughout the anterior compartment and confers retinal fate to the tissue through its induction of the RDGN (Czerny et al., 1999 Kronhamm et al., 2002). Of Ey functions in the developing eye, it primarily serves to promote the expression of Sine oculis (So), Eyes absent (Eya) and Dachschund (Dac), which together form a tripartite transcription factor that, in conjunction with Ey, stimulate the expression of Ato, introducing the hallmark of proneural activity and the transition to the MF (Zhang et al., 2006; Tanaka-Matakatsu and Du, 2008; Tanaka-Matakatsu et al., 2014).

\section{The morphogenetic furrow forms at the interface of the two compartments.}

Situated between the anterior and posterior compartments is the morphogenetic furrow (MF), a dorsoventral groove that forms from the constriction of cells at their apical surfaces and along their apicobasal axes (Fig. 4A). Cytoskeletal reorganization is often associated with morphogenesis, as with the classical examples of gastrulation and neurulation (Sawyer et al., 2010; Martin and Goldstein, 2014). Drosophila retinogenesis is included with these examples, although it is not clear whether apical constriction is a requirement for ensuing morphogenesis. However, apical constriction likely impacts developmental outcomes through the concentration of receptors and other membrane-embedded proteins to their respective membrane compartments and potentially serves to modulate the available cellular surface area, potentially impeding (or accelerating) the diffusion of morphogens (Wolff and Ready, 1991). The maturation of both Hh and Dpp feature post-translational modification: for $\mathrm{Hh}$, the incorporation of a lipid moiety, and for Dpp, proteolytic cleavage to free the C-terminus (Lee et al., 1994; Porter et al., 1995; Cui et al., 1998; 
Kunnapuu et al., 2009). The incorporation of a lipid moiety into the Hh morphogen likely serves to enhance membrane interaction and slow diffusion (Porter et al., 1996). Thus, it is plausible that in the eye disc, apical constriction might serve to modulate diffusion of the morphogens Hh and Dpp, allowing the MF to serve as a sink for free, extracellular morphogen (Chen and Struhl, 1996).

The progressive differentiation of cells within the eye has allowed this tissue to become the paradigm of signaling studies. Most tissues develop continuously, limiting observations to only the particular stage of development that corresponds to the time of observation. However, during the transit of the MF, the Drosophila retina is comprised of a full range of developmental stages. Thus, the same tissue features an equipotent anterior compartment and an actively differentiating posterior with ommatidia that show a state of maturity that corresponds to the duration since the passage of the MF.

\section{Hh and Dpp are the primary morphogens of retinogenesis.}

The conserved pathways of Hh and Dpp (both are named after the secreted ligand) are critical to regulating the timing and organization of Drosophila eye development, as discussed below in detail. The Hh pathway operates akin to that of Notch and Wg in that signal activation reverses the effects of a basal transrepressor complex (via $\mathrm{Su}(\mathrm{H})$ in Notch and TCF/LEF in Wg). For the Hh pathway, the zinc finger Cubitus interruptus (Ci) serves as the repressor-cum-activator, and its activity is regulated by its phosphorylation status, which determines whether the transcription factor becomes proteasomally cleaved into a truncated transrepressor (Cirep) or maintains its fulllength transactivator form (Ciact) (Aza-Blanc et al., 1997). In the pathway's basal state, the transmembrane receptor Patched (Ptc) prevents the surface localization of vesicles that contain the transmembrane protein Smoothened (Smo) through an indeterminate mechanism. Costal-2 (Cos2), an atypical kinesin, acts as a scaffold to sequester $\mathrm{Ci}$ and its positive regulator Fused kinase (Fu) (Sisson et al., 1997; Robbins et al., 1997). In the absence of Hh, the association of Cos2 with Ci facilitates multiple phosphorylations of Ci by PKA, CK1, and GSK3- $\beta$, resulting in the production of a 
phosphodegron that is recognized by the E3 ubiquitin ligase F-box component Supernumerary limbs (Slmb), allowing Ci to be proteasomally cleaved into its N-terminal repressor form (Chen et al., 1999; Price and Calderon, 2002; Jia et al., 2002; Jiang and Struhl, 1998). Cirep maintains DNAbinding capacity, but lacks the transactivation domain of its full-length form, facilitating a block to target gene expression (Aza-Blanc et al., 1997). Upon interaction of Hh with a Ptc-receptor complex, Smo vesicles are permitted to dock and integrate with the cell membrane (Taipale et al., 2002). Smo then recruits $\operatorname{Cos} 2$ to its intracellular tail, eliciting the activation of Fu, which subsequently phosphorylates $\mathrm{Ci}$, making the protein refractory to modification by PKA, CK1 and GSK3- $\beta$ (Lum et al., 2003; Jia et al., 2003; Ruel et al., 2003). Thus, Ci is spared partial degradation, and the full length, transactivator form is able to accumulate and associate with target genes in the nucleus (Motzny and Holmgren, 1995; Chen et al., 1999). Interestingly, some situations, including Drosophila retinal patterning, require only the removal of Cirep to enable target gene expression; Ciact has no role in positively regulating gene expression in such scenarios (Methot and Basler, 1999; Pappu et al., 2003).

Dpp is the Drosophila ortholog of vertebrate Bone morphogenetic proteins-2 and-4, members of the TGF- $\beta$ superfamily. As such, Dpp displays a prominent role in establishing tissue polarity throughout development (Terracol and Lengyel, 1994; Jiang and Struhl, 1996; Singer et al., 1997). Dpp signaling is initiated when the ligand Dpp precipitates the interaction of a heterotetrameric receptor complex that forms from type I and type II receptors. Oligomerization of receptors was first shown in mammalian cell culture, and later supported by Drosophila studies (Chen and Derynck, 1994; Weis-Garcia and Massague, 1996; Simin et al., 1998). In the Drosophila eye, Thickveins (Tkv) and Punt are the type I and type II receptors, respectively (Brummel et al., 1994; Ruberte et al., 1995). The formation of the ligand-receptor complex activates the intracellular kinase activity of the type II receptor, which phosphorylates the type I receptor, which, in turn, phosphorylates the soluble, cytosolic SMAD family protein (Wrana et al., 1994; 
Ventura et al., 1994; Kretzchmar et al., 1997; Macias-Silva et al., 1996). In Drosophila, Mothers against decapentaplegic (Mad, or pMad to designate the active phospho-form) is the cytosolic target of the activated type I TGF- $\beta$ receptor. pMad, in conjunction with the SMAD4 ortholog Medea (Med), modulates the activities of target genes (Raftery et al., 1995; Sekelsky et al., 1995). The repressor Brinker (Brk) is the primary target of pathway activity (Minami et al., 1999; Jazwinska et al., 1999). pMad and Med form a nuclear complex with the repressor Schnurri (Shn) to temporarily silence $b r k$, de-repressing Brk targets (Marty et al., 2000; Torres-Vazquez et al., 2000). In addition, pMad-Med heterodimers likely function in the absence of Shn, though potential co-modulators have not yet been identified.

\section{Hh and Dpp regulate the rate of eye development.}

Though Ato is required for MF movement and differentiation, the continual anterior migration of the morphogenetic furrow is driven by the combinatorial activity of Hh and Dpp (Fig. 4A; Heberlein et al., 1993; Ma et al., 1993; Heberlein et al., 1995). Differentiation proceeds with the induction of $\mathrm{Hh}$ in cells along the posterior margin of the eye disc, likely as a result of hormonal stimulation (Dominguez and Hafen, 1997). Hh serves to elicit the induction of two genes from signal-receiving cells: ato and $d p p$. Of these, we will first focus on the defined roles of $d p p$ as it relates to MF transit. Dpp is secreted uniformly from cells of the MF and acts as a long-range potentiator of RDGN activity within the anterior compartment (Heberlein et al., 1993; Strutt and Mlodzik, 1997; Blackman et al., 1991; Firth and Baker, 2009). RDGN activity directs the expression of Ato; thus, failure to respond to Dpp results in postponement of the genetic programming of the MF (Burke and Basler, 1996; Greenwood and Struhl, 1999; Li et al., 2013). Prior to Dpp signaling, cells are maintained in an undifferentiated state by Wg signaling that emanates from the anterior and dorsoventral margins (Treisman and Rubin, 1995). Wg promotes expression of homothorax, $h t h$, which acts as one component of a tripartite repressor complex with Teashirt (Tsh) and the eye-

specification protein Ey (Bessa et al., 2002). Presumably, this complex serves the dual function of 
silencing genes necessary for RDGN activity in addition to the titration of Ey from its transactivational targets. In response to Dpp, hth becomes silenced, freeing Tsh and Ey to activate eya, so and dac, in turn, activating Ato at the posterior margin of the anterior compartment (Bessa et al., 2002; Firth and Baker, 2009).

In addition to its expression along the posterior margin, Hh is secreted from differentiated (those that express the pan-neural marker Elav), non-R8 retinulae (Fig. 4A; Ma et al., 1993). Thus, the recruitment of secondary photoreceptors also serves to stimulate the establishment of increasingly further anterior sources of Hh secretion. Hh is thought to act at shorter range than Dpp, potentially acting less as a direct activator of key components of the RDGN, but rather instigating rapid restructuring of the network (Firth and Baker, 2009; Baker and Firth, 2011; Atkins et al., 2013). As with removal of the Dpp response, loss of Hh imposes significant delay on the induction of Ato, thus delaying proper progression of MF-related gene expression (Dominguez, 1999; Greenwood and Struhl, 1999). Additionally, Dpp contributes to instituting a G1 cell-cycle arrest within cells proximally anterior to the MF, preparing the tissue for uniform entry into the differentiation program (Firth et al., 2010).

\section{Ato proceeds through four discrete stages of expression.}

The MF is the site of notable, fast-paced changes to gene expression. The induction of Ato demarcates the transition zone from the PPN to the MF and the expression pattern of Ato typifies the continued transition that is observed during this milestone, as Ato quickly progresses through four discrete stages of expression, characterized from anterior to posterior (Baker et al., 1996). Stage- 1 is identified as a broad, dorsoventral band of low-level expression, which segues into stage2, where well-spaced clusters of up to 20 cells express Ato more strongly and are known as intermediate groups (IGs; Fig. 5A). Stage-2 clusters then become isolated from the body of stage-1 and -2 expression and restricted to increasingly smaller subsets of cells from the IGs (stage-3; Fig. 5A). Lastly, stage-4 is achieved once only one Ato-positive cell from each IG remains; such cells 
continue on to become R8s (Fig. 5A; Jarman et al., 1994; Jarman et al., 1995). Thus, the loss of Ato results in the loss of R8s and the failure to recruit any secondary photoreceptor types (Jarman et al., 1994). Additionally, ato mutant flies lack the successful anterior-posterior transit of the MF (Jarman et al., 1995).

\section{ato is regulated by two separable enhancers.}

ato transitions through its complex expression pattern (stages 1-4) via activity on two separable eye-specific enhancers; a $2.1 \mathrm{~kb}$ enhancer that starts $9.3 \mathrm{~kb}$ upstream of the ato transcription start site and a 5.8kb enhancer located proximally $3^{\prime}$ of the transcription unit (Fig. 5B; Sun et al., 1998). These enhancers, more commonly referred to as the 5' and 3' enhancers, correspond to specific stages of ato expression, as previously defined. Stage-1 expression, the furthest anterior, earliest domain of ato expression is directed by the binding of at least four members of the RDGN: Ey, So, Eya, and Dac (Fig. 5B; Zhang et al., 2006; Tanaka-Matakatsu and Du, 2008; Tanaka-Matakatsu et al., 2014). Recent reports indicate that Ey binding to this enhancer requires the E protein Daughterless (Da), although da-null tissue exhibits no apparent ato-3' induction defects (Tanaka-Matakatsu et al., 2014; Melicharek et al., 2008). Thus, stage-1 ato is indirectly linked to the timely response to $\mathrm{Hh}$ and Dpp signals through their elicitation of the RDGN (Fig. 5B). Stages-2 through -4 require activity on the 5' enhancer. 5'-ato requires autoregulation wherein Ato, as a heterodimer with the bHLH Da, stimulates continued expression of Ato, though it is untested whether retinal autoregulation of ato is through direct or indirect means (Fig. 5B;

Brown et al., 1995; Sun et al., 1998; Baker et al., 1996).

Although ato-3' is initially expressed broadly, RNA in situ hybridization reveals that the posterior margin of the 3 ' enhancer expression domain is subject to variation along the dorsoventral margin, as made distinct by the formation of a regular, wave-like phasing to the posterior margin (Sun et al., 1998). The 5' enhancer, as a function of its autoregulatory requirement, is activated posterior of initial ato-3' activity. Upon initial induction, $5^{\prime}$-ato drives 
expression within large clusters that correspond to the location of IGs (Fig. 5B). As the MF progresses further anterior, the 5' enhancer becomes restricted to only R8s before ultimately losing all activity (Fig. 5B; Sun et al., 1998). Note that immunohistochemical detection of 5'-ato-lacZ activity frequently results in the staining of all R8s through the posterior compartment, as is likely caused by the perdurance of $\beta$-Gal in Drosophila tissues. Although RNA in situ analysis fails to directly illustrate such, cross referencing RNA in situ data with that of immunostaining reveals that it is likely that ato-3' and 5'-ato overlap within IGs. The individual role of each enhancer is recapitulated in experiments wherein each enhancer is used to individually drive synthetic ato coding region constructs. In isolation, neither construct could rescue the functionally deficient ato ${ }^{1}$ mutation, but together ommatidial formation was restored across the eyefield (Sun et al., 1998).

\section{Ato is required for induction of Senseless.}

Although ato is the gene whose expression is exquisitely patterned within the MF, proper retinogenesis requires that Ato induce the neural specification gene sens. Sens expression initiates within Ato IGs, and is likewise restricted to R8s where it is required for R8 differentiation (Nolo et al., 2001; Frankfort et al., 2001; Pepple et al., 2008). In tissue that lacks Sens, photoreceptors form (Frankfort et al., 2004). However, sens mutant ommatidia fail to properly develop, owing to a complete loss of R8s. The fate of the R8 is likely immediately usurped by that of R2/5 (Frankfort et al., 2001; Pepple et al., 2008). Furthermore, the loss of R8 may disable several further steps of ommatidial recruitment and assembly, resulting in an average number of 5 retinulae per sens mutant facet (Frankfort et al., 2001). Additionally, Sens exhibits positive feedback on ato. With the loss of Sens, Ato expression initiates normally and patterns into IGs. However, Ato fails to properly transition into R8s that are capable of maintaining Ato expression (Frankfort et al., 2001).

\section{Ato elicits Notch signaling within the MF.}

Notch signaling within the MF has been described as biphasic in that first, Notch is required to facilitate ato autoregulation on the $5^{\prime}$ enhancer and second, via $\mathrm{Su}(\mathrm{H})$, Notch induces the 
restriction of proneural competency to a single cell. The first step, termed proneural enhancement, has been speculated to occur through several possible mechanisms: first that Notch might directly enhance transcriptional output of Ato, presumably through the $5^{\prime}$ enhancer, second that Notch might post-translationally modify Ato to enhance function, or stimulate some yet unidentified modifier of Ato function, and third, that Notch might repress a repressor of ato (Baker and Yu 1997). Unsurprisingly, the third option has gained greatest traction as the most likely possibility. Furthermore, the MF is preceded by the expression of the repressor Hairy (h) and the bHLH antagonist Extramacrochaetae (Emc) (Brown et al., 1991; Brown et al., 1995).

\section{Emc and Hairy Repress ato anterior to the MF.}

Emc was originally identified as having a role in restricting the number of chaetae that form during development; a phenotype that was later traced to its role in promoting the lateral inhibition of neural potency from PNCs. Emc is a class V HLH that is orthologous to mammalian Inhibitor of differentiation, Id. Class V HLHs inhibit bHLH function, presumably through promiscuous heterodimerization with bHLHs and subsequent disruption of DNA binding, which requires functional hetero- and homodimers (Ellis et al., 1990; van Doren et al., 1991). It is hypothesized that Emc, as with its mammalian orthologs, establishes a rheostatic threshold of bHLH activity that must be overcome before permitting differentiation, thus facilitating fine-tuning of a binary cell fate decision (Troost et al., 2015). Emc is widely expressed throughout Drosophila development (Bhattacharya and Baker, 2011). During retinogenesis, Emc is expressed throughout the anterior eye compartment and in most undifferentiated cells of the posterior, with notable exemption from the Ato-positive cells of the MF (Bhattacharya and Baker, 2011).

Unlike emc, hairy encodes a bHLH repressor capable of DNA binding and recruitment of three corepressors: Gro, C-terminal binding protein, and Sir2 (Ohsako et al., 1994; Wainwright and Ish-Horowicz, 1992; Paroush et al., 1994; Poortinga et al., 1998; Rosenberg and Parkhurst, 2002). However, Hairy expression is more limited, reflecting a less diverse portfolio of situations in which 
it is employed. In the developing eye, Hairy is expressed in the anterior compartment downstream of Dpp signaling, but requires Hh signaling for its downregulation (Brown et al., 1991; Li et al., 2013; Fu and Baker, 2003). In fact, the domain of the PPN is defined by H expression, which is promptly lost when Notch initiates in the MF (Baonza and Freeman, 2001). Classically, Hairy serves a role in direct repression of the bHLH proneurals of the Asc complex in sensory organ formation in the wing and leg (Fisher and Caudy 1998).

Initial studies of these repressors indicated that the two, Emc and Hairy, act in conjunction to slow the progression of the MF; a reasonable hypothesis in that the presence of Emc could titrate proneural bHLH activity and allow Hairy to directly repress proneural targets such as Ato (Brown et al., 1995). In line with this proposed bimodal impedance to MF movement, loss of either Emc or Hairy is insufficient to loosen the reins of MF movement, and, in juxtaposition, force-expression of either hindered entry into the MF (Brown et al., 1995; Brown et al. 1991; Bhattacharya and Baker, 2012). In a turnaround of previous findings, recent studies have shown that individual removal of either Emc or Hairy has differential effects on precocious advancement of the MF (Bhattacharya and Baker, 2012). Loss of Hairy alone reveals little change to the expression of MF-associated factors, with the exception of enhanced report from the 5'-ato enhancer (Spratford and Kumar, 2013). Alternately, emc-null tissue exhibits rapid anterior movement of the MF, as detected by apical constriction, Dpp-reporter expression, and greatly enhanced Hh response (Bhattacharya and Baker, 2012; Spratford and Kumar, 2013). Although emc-null clones appear to lack forward advancement of 5'-ato, dual emc-hairy mutants exhibit anteriorly accelerated Ato expression, in addition to all other gene expression changes associated with only Emc loss (Brown et al., 1995), indicating that Ato is likely produced in emc mutants, but only from the 3' enhancer.

Recent analyses have probed the potential for cross-regulation between emc and hairy, but have yielded little explanatory insight. hairy-null tissue yields only minor defects in emc expression (Bhattacharya and Baker, 2012). In the hands of different labs, emc mutants elicit drastically 
different results, despite the use of the same emc ${ }^{A P 6}$ allele and anti-Hairy antibodies. The Baker lab reports that Hairy expression is only minorly affected whereas the Kumar lab reports drastic loss of hairy (Bhattacharya and Baker, 2012; Spratford and Kumar, 2013). The approach of each lab was similar, although the position of the mutant clones may dictate the observed difference. In Baker's report, the clones occur within the midst of the eye disc, away from margins whereas Kumar's report makes use of a large mutant clone that borders the dorsal margin of the developing eye. Past studies have indicated that the contiguity with the margins of the eye disc can elicit different affects in clones than in those that are fully encircled by WT tissue (Mardon et al., 1994; Treisman and Rubin, 1995).

Ato's dimerization partner Da is subject to regulation by Emc although Da is not regulated by Hairy. In the absence of Emc, Da expression increases dramatically in the eye disc and throughout other imaginal discs (Bhattacharya and Baker, 2012; Bhattacharya and Baker, 2011). To demonstrate that the upregulation of Da is responsible for MF acceleration, dual mutants of emc and $d a$ exhibit no advanced MF movement and closely resemble $d a$-null tissue (Bhattacharya and Baker, 2011).

The transition from the PPN to the MF requires the downregulation of emc and hairy (Bhattacharya and Baker, 2011; Brown et al., 1991). Notch signaling is the likely potentiator of this transition given that Ato becomes active at this time, has brief coexpression with both repressors, and induces Notch signaling (Brown et al., 1995; Baonza and Freeman, 2001; Sun et al., 1998). Furthermore, removal of Notch results in the continued expression of Hairy throughout the MF and posterior compartment - a phenotype that is coupled with the continued, low-level, unpatterned expression of Ato (Baonza and Freeman, 2001; Fu and Baker, 2003). Likewise, ectopic expression of $\mathrm{Dl}$ anterior to the MF results in precocious loss of $\mathrm{H}$ and the advancement of Ato further anterior into the PPN, whereas diminished Notch signaling, through the removal of the $\mathrm{Su}(\mathrm{H})$ coactivator mam, has no effect on Emc within the MF (Baonza and Freeman, 2001). Force-expression of Dl 
negatively imposes on Emc, but it is currently hypothesized that emc is downregulated within the MF as a noncanonical, $\mathrm{Su}(\mathrm{H})$-independent form of Notch signaling (Bhattacharya and Baker, 2009) In total, the downregulation of both emc and hairy is, at the least, associated with the onset of Notch signaling, and hairy requires the Notch receptor for its downregulation. The genetic evidence supports that in WT, Emc throttles the Hh response while Hairy prevents activation of 5'ato. Thus, the activation of Notch serves a dual role, in addition to repressing emc and hairy, Notch allows Da expression to rise as a response to removal of Emc. The increase in Da also potentially aids in the autoregulation of ato, which can proceed in the absence of hairy. Interestingly, recent findings suggest that neural specification is the result of the activity of Da homodimers - that proneural bHLHs function less as heterodimerization partners with Da than as interaction partners with Emc, to prevent Emc-Da dimerization (Troost et al., 2015). The removal of Asc function results in failed SOP formation and bristle-less adult flies. However, the removal of both Asc and Emc restores SOP formation. It remains yet to be tested in the eye whether flies mutant for both ato and emc are capable of forming R8s.

\section{$E(s p l)$ locus encodes seven bHLH-O repressors.}

After downregulation both emc and hairy in the MF, Notch signaling is utilized to restrict Ato to its late-stage expression into R8s. The Enhancer of split locus is comprised of genes that encode four repressors of the bearded family, a protease inhibitor, seven bHLH-O repressors, and the corepressor Groucho (Gro; Fig. 6A). The bHLH-0 repressors include $m \delta, m \gamma, m \beta, m 3, m 5, m 7$ and $m 8$ (Klambt et al., 1989; Knust et al., 1992). The overall structure of the E(spl) bHLH-Os is conserved (Fig. 6B,C; CtDs, Delidakis and Artavanis-Tsakonas, 1992). All are comprised of a basic domain to facilitate DNA binding, followed by a helix-loop-helix that facilitates homo- and heterodimerization, an Orange helix-loop-helix that facilitates interaction with non-bHLH-O proteins and an ultimate tetrapeptide of WRPW that allows Gro interaction (Fig. 6B; Tietze et al., 1992; Taelman et al., 2004; Zarifi et al., 2012; Paroush et al., 1994; Jennings et al., 2006). Despite 
well-conserved core bHLH-O structures, the CtDs of the E(spl) repressors display diversity in length and likely in function. As such, four of seven E(spl) repressors encode C-terminal kinase motifs, as do orthologs in other species, including E(spl)M8 corollary Hs-HES6 (Majot et al., 2015).

Although the seven members of the $E(s p l)-C$ are remarkably well conserved in both content and position across Drosophila, this multitude of bHLH-O genes is observed only in the closest Drosophila ancestors (Baker et al., 2011). Studies of E(spl) across Diptera and more ancestral lineages suggest that the duplication of this locus arose within the past $200 \mathrm{Myr}$ from a single $E$ (spl) progenitor (Duncan and Dearden, 2010; Schlatter and Maier, 2005). This conclusion is further supported by phylogenetic comparisons of vertebrate and invertebrate Hes members, which indicate a generalized distinction between the two groups (vertebrate versus invertebrate), demonstrating that only the broad structural and functional perspectives of $\mathrm{E}(\mathrm{spl})$ may be conserved (Duncan and Dearden, 2010). As such, the study of Drosophila E(spl) proteins is apt to reveal broadly applicable principles regarding function and regulation. One such principle first elucidated in Drosophila is the conserved use of phosphorylation as a means of post-translational modulation of $\mathrm{E}(\mathrm{spl})$ repressor function. The following pages explore this conserved role of phosphorylation, primarily through the scope of studies into E(spl)M8.

\section{$\mathrm{E}(\mathrm{spl})$ represses ato in the $\mathrm{MF}$.}

As previously addressed (on pg. 8), E(spl) genes are expressed in response to proneuraldriven Notch signaling (Jennings et al., 1994). Similar to their role(s) in lateral inhibition during SOP formation, $E(s p l)$ is utilized in the eye to restrict ato from broader IG expression to only the R8. The enhancer architecture throughout the locus is consistent with a role in Notch signaling; many $E(s p l)$ genes are transcriptionally activated through the binding of two, oppositely-oriented $\mathrm{Su}(\mathrm{H})$ binding sites termed Su(H)-paired sites (SPS) (Bailey and Posakony, 1995; Nellesen et al., 1999; Maeder et al., 2007). SPS sites are often coupled with proneural-binding E boxes, functionally integrating the activity of proneural genes and Notch signaling (Cave et al., 2005). Unsurprisingly, 
reporter assays indicate that $E(s p l)$ genes exhibit particularly strong sensitivity to Notch signaling, responding more quickly than other Notch-responsive genes, implicating $E(s p l)$ as a critical component of the Notch signal cascade (Housden et al., 2013).

Following the removal of Emc and Hairy from the MF, ato achieves autoregulation within IGs (Brown et al., 1995; Bhattacharya and Baker, 2012, Spratford and Kumar, 2013). The positions of the IGs, though likely dictated by a dorsoventral Hh gradient, are enforced by the expression of $E(s p l)$, which are first observed within the MF and throughout the posterior retinal compartment (Lubensky et al., 2011; de Celis et al., 1996; Li and Baker, 2001; Ligoxygakis et al., 1998, Baker and Yu, 1997). However, individual E(spl) genes are differentially expressed throughout eye development. RNA in situ reveals that $E(s p l) m \delta, m \gamma, m \beta, m 7$ and $m 8$ are expressed within the MF and $m \delta, m \gamma$ and $m \beta$ accumulate within undifferentiated cells of the posterior compartment (de Celis et al., 1996). The antibody $\mathrm{mAb323}$ recognizes several $\mathrm{E}(\mathrm{spl})$ proteins, including $\mathrm{M} \delta, \mathrm{M} \gamma, \mathrm{M} 3$, and $\mathrm{M} \beta$ and, more weakly, M8. Thus, mAb323 confers detection of generalized Su(H)-dependent Notch activity within imaginal tissues (Jennings et al. 1994).

\section{E(spl) disrupts proneural function.}

It was presumed that the mode of repression by genes of the $E(s p l)$ locus would be to either homo- or heterodimerize and bind target DNA sites to which Gro could be recruited, suggesting that E(spl) might repress transcription of proneural genes and/or the targets of proneural genes (Fig. 6D). To validate this possibility, it has been reported that $\mathrm{E}(\mathrm{spl})$ dimers bind to specific E-box variants ( $E_{b}$ boxes), as well as $C$ and $\mathrm{N}$ boxes, facilitating transcriptional repression through direct DNA-binding (Jennings et al., 1999; ). Furthermore, E(spl)-binding motifs are frequently in the same enhancer modules as $\mathrm{E}_{\mathrm{a}}$ boxes, which have been shown to bind Da-proneural heterodimers (Culi and Modolell, 1998; Singson et al., 1994). In contrast to this simple model, DNA-binding was shown to be unnecessary for sufficient repression of target genes. In force-expression experiments, basic domain mutants were shown to effectively repress bristle formation (Nakao and Campos- 
Ortega, 1996; Giebel and Campos-Ortega, 1997). Furthermore, force-expression of an E(spl)m7 variant that housed a mutated basic domain and replaced the co-repressor-binding WRPW motif with a VP16 transactivation domain was able to induce excess bristle formation, but only in flies that expressed the SOP proneural Sc, indicating that $\mathrm{E}(\mathrm{spl})$ can be indirectly targeted to enhancers through a tethering mechanism via interaction with proneurals (Fig. 6D; Giagtzoglou et al., 2003). Further efforts in both flies and Xenopus suggest that the Orange domain of Hes proteins permits protein-protein interaction with the transactivation domain of proneurals (Giagtzoglou et al., 2005; Zarifi et al., 2012).

\section{$E(s p l)^{D}$ encodes a hypermorphic truncation of E(spl)M8.}

Molecular characterization of the dominant gain-of-function $E(s p l)^{D}$ allele has revealed the potential for sophisticated regulation of Hes proteins and alternate modes of Hes-mediated repression. $E(\mathrm{spl})^{D}$ was originally isolated from its strong interaction with the mutant receptor $N^{s p l}$ and further efforts later identified the $N^{s p l} ; E(s p l)^{D}$ interaction to be enhanced by reduced dosage of either ato or $d a$ (Welshons, 1956; Nagel and Preiss, 1999). $N^{s p l}$ results from altered fucosylation of EGF repeat 14 of the receptor, causing an apparent enhancement in either Notch activity or sensitivity (Li et al., 2003). Specifically, $N^{s p l}$ disrupts R8 and R7 specification, processes that both rely on appropriate Notch pathway activity. $N^{s p l}$ elicits R8s that fail to maintain their differentiated fate, and supernumerary R7s per ommatidia(Li et al., 2003). The $N$ spl; $E(s p l)^{D}$ interaction further disrupts R8 specification - fully in males which are hemizygous for $N^{s p l}$ and partially in transheterozygous females (Welshons, 1956; Nagel and Preiss, 1999). Furthermore, E(spl)D revertants demonstrate that (at least some) genes of the $E(s p l)$ locus are neurogenic, substantiating a role for $\mathrm{E}(\mathrm{spl}) \mathrm{M} 8$ in neurogenesis and dismissing the possibility that $E(s p l)^{D}$ is an anomalous neomorphic mutation that has no bearing on WT gene function.

The $E(s p l)^{D}$ allele specifically affects $m 8$, producing a truncated protein that lacks the CtD and Gro-interaction motif (Fig. 6B; Tietze et al., 1992; Nagel et al., 1999). In spite of the loss of Gro- 
binding, $E(s p l)^{D}$ acts as a hypermorph (Welshons, 1956). Subsequent exploration into this perceived paradoxical phenotype revealed that the CtD plays an autoinhibitory role. Yeast-twohybrid protein interaction assays have revealed that native-state M8 only weakly interacts with Ato, whereas M8*, the protein product of $E(s p l)^{D}$, shows strong affinity for such a target, demonstrating that M8 interaction with Ato is disrupted by the presence of a native-state M8-CtD (Fig. 6B; Alifragis et al., 1997; Nagel and Preiss, 1999; Karandikar et al., 2004). From this data, it became clear that $E(s p l)^{D}$ had imparted upon researchers a window into the role of posttranslational modification of M8 and other E(spl) proteins.

\section{CK2 phosphorylates E(spl).}

Further examination of the M8-CtD sequence, which is missing from M8*, revealed a conserved stretch of several Ser residues embedded within phosphorylation motifs of the protein kinases MAPK and CK2 (Fig. 6C). A yeast-two-hybrid screening for interaction partners of Drosophila CK2 $\alpha$, the kinase's catalytic subunit, against a Drosophila embryonic cDNA library revealed CK2 to interact with the three E(spl) members M5, M7 and M8 (Trott et al., 2001). All three genes harbor a conserved CK2 phosphorylation motif, and two, $m 5$ and $m 8$, encode conserved MAPK consensus sites. Follow-up studies confirmed that, in vitro, CK2 phosphorylates all three.

Unfortunately, deconvolution of the CK2-E(spl) relationship has been encumbered by several severe limitations, chief of which include the relative closeness of genes within the $E(\mathrm{spl})$ locus, the proximity of the cell-essential co-repressor gro, and the centromere-proximal location of $c k 2 \alpha$. The close-spacing of genes within this locus (7 bHLH-O repressors and another 4 beardedfamily member genes across less than $53 \mathrm{~kb}$ ) has hindered the development of individual $E$ (spl) mutants given that chemical and $\mathrm{x}$-ray mutagenesis frequently elicit genomic lesions that span multiple $E(s p l)$ members. This is a problem that is further exacerbated by the indefinite association between specific enhancer modules and particular genes of the locus (Bailey and Posakony, 1995; Maeder et al., 2007; Cooper et al., 2000). In most loci, genes typically display expression changes 
that result from enhancers that are within $10 \mathrm{~kb}$ of the transcription start site (Berman et al., 2004). Problematically, such a range spans multiple coding members within the $E(s p l)$ locus. Alternately, the possibility of enhancer 'entanglement' between the various $E(s p l)$ and bearded family members might effectively be the cause of the conserved closeness and position of these genes throughout Drosophila and closely-related taxa. Additional difficulties arise from $m 8$ 's proximal location to gro and their separation by a chromatic insulator (Schaaf et al., 2013). Any attempt to isolate lesions that affect $m 8$ have invariably impacted $g r o$ as well, preventing, to date, $m 8$-mutant analysis that is independent of complications caused by alterations to gro.

\section{Phosphorylation of Ser ${ }^{159}$ likely facilitates repression by E(spl)M8.}

The most insightful work into the CK2-E(spl) relationship has been through the study of M8 phosphovariants. Through a technique that is customary to the study of phosphoregulation, a phospho-accepting residue is mutated to either an acidic residue to simulate the charge character of an attached phosphate, or a neutral residue, such as Ala, to disallow modification via kinase activity. Whereas force-expression of $m 8^{W T}$ within Ato-positive IGs revealed no perturbation to R8 formation, similar expression of either CK2 phosphovariant (with mutation induced at Ser ${ }^{159}$ ) elicited defects to adult eye morphology (Karandikar et al. 2004). Immunostaining indicates that, in the case of $m 8^{S 159 D}$, the eye perturbation results from the loss of Ato during its transition from IG to R8, a phenotype akin to that observed in sens mutants (Frankfort et al. 2001). The phosphomimetic result suggests that phosphorylation of Ser ${ }^{159}$ likely facilitates M8's antagonism of proneural function (Fig. 6B).

\section{Phosphorylation of E(spl)M8s159 relieves autoinhibition.}

Thus, M8 is held in a state of autoinhibition wherein the CtD occludes the exposure of contact sites between the bHLH-O domains and Ato (Fig. 6B). In an elegant set of experiments, the core of M8 (the bHLH-O) was shown to preferentially interact with the WT CtD over the phosphomimetic form (Kahali et al., 2010). Furthermore, the reduced eye caused by force- 
expression of $m 8^{S 159 D}$ is potently rescued by concurrent expression with transgenic $m 8-C t D$. No such rescue is observed with expression of $m 8^{S 159 D}$ and $m 8-C t D^{S 159 D}$, further establishing a role for Ser ${ }^{159}$ in autoinhibition and strongly suggesting that this autoinhibition is relieved upon phosphorylation of this residue by CK2. Force-expression during SOP formation results in bristle loss, recapitulating those that are observed in the eye. Counter to eye formation, SOPs display sensitivity to force-expression of $m 8^{W T}$, indicating that phosphorylation is a nonrequirement for repression, a finding that is perhaps reflected in the multiple possible modes of repression by E(spl) proteins, either DNA-binding independent or Gro-independent (Ligoxygakis et al., 1998; Nagel and Preiss 1999). Regardless, coexpression with m8-CtD was able to suppress the baseline loss of SOPs observed with expression of only $m 8^{W T}$. In contrast, coexpression of $m 8-C t D^{S 159 D}$ was unable to suppress the presumed repression by $m 8^{W T}$. As a final test of the autoinhibitory model, the $N^{s p l} ; E(s p l)^{D}$ interaction was subjected to force expression of WT and phosphomimetic variants of the $m 8-C t D$. Consistent with previous findings, expression of $m 8-C t D$ mildly rescues the eye defect whereas $m 8-C t D^{S 159 D}$ exhibits no such modulation (Kahali et al. 2010).

\section{$E(s p l)^{D}$ is active earlier than $m 8^{S 159 D}$.}

Experiments with $m 8^{5159 D}$ have clarified a role for the CtD in the autoinhibition of M8. However, comparisons between the analyses of $m 8^{S 159 D}$ and $E(s p l)^{D}$ are complicated by the difference in genetic background in which the two variants of $m 8$ act; $m 8^{S 159 D}$, when driven in IGs and R8s, elicits eye defects in an otherwise WT background whereas $E(s p l)^{D}$ is only reported to perturb eye patterning in combination with $N^{s p l}$. $E(s p l)^{D}$ encodes multiple defects that affect the expression of its protein and that of gro; thus, a transgenic variant of $E(s p l)^{D}$ was produced to limit the scope of studies to only the effects of M8*, where necessary. Attempts to forcibly express $m 8^{*}$ in IGs and R8s have failed to affect R8 patterning (Kahali et al., 2009). Furthermore, the effects of $m 8^{S 159 D}$ are neutralized when Gro-binding is abrogated through the removal of the terminal WRPW motif, whereas $E(s p l)^{D}$ is most intriguing for the fact that it potently disrupts proneural function 
despite lacking Gro-interaction (Kahali et al., 2009). The cause of $E(s p l)^{D^{\prime}}$ s high-level repression of proneural function rests in the timing of its activity. In the $N^{s p l}$ background, force-expression of $m 8^{*}$ only elicits R8-patterning defects when driven during early Ato patterning, prior to late-stage IG and R8 formation.

\section{Roughened eye facilitates ato autoregulation.}

The rotund locus encodes two overlapping zinc finger transcription factors - the transactivator rotund $(\mathrm{rn})$ and the transrepressor roughened eye (roe). Although both genes share exons that encode the same C-terminal region that includes DNA-binding capability, each are expressed from alternate promoters that direct the expression of divergent $\mathrm{N}$-terminal domains (St. Pierre et al., 2002). Furthermore, the two promoters drive divergent expression, displaying no overlap in the same tissues. Thus, Rn and Roe are not competing for DNA-binding nor are they directly opposing each other. roe is expressed in a smaller subset of contexts than $r n$, primarily within the MF of the eye and in R1/6 retinulae (St. Pierre et al., 2002; Melicharek et al., 2008; del Alamo and Mlodzik, 2008). roe interacts strongly with ato to elicit reduced adult eyes (Melicharek et al., 2008). Similarly, roe enhances the eye phenotype of $N^{s p l}$ flies (Brand and Campos-Ortega, 1990). In the absence of Roe, ato fails to commit to autoregulation, despite appropriate activation of its 3' enhancer (Melicharek et al., 2008). Furthermore, both EMSA and ChIP assays reveal that Roe binds to the enhancer of $E(s p l) m \delta$, which encodes a bHLH repressor that is expressed in the MF resultant of Notch-Su(H) activity (del Alamo and Mlodzik, 2008). Binding of Roe occurs independently of that of $\mathrm{Su}(\mathrm{H})$ but Roe affinity for the enhancer is not diminished in the presence of $\mathrm{Su}(\mathrm{H})$, suggesting that Roe may attenuate MF-specific expression of negative regulators of ato within the MF (del Alamo and Mlodzik, 2008). Such a role agrees with expression data of E(spl) repressors in the MF, as several E(spl)bHLHs, as independently labeled by multiple antibodies, appear to be first expressed anterior to IGs before being lost from clustered regions where stage-2 ato becomes most strongly expressed (Baker et al., 1996). Interestingly, roe expression within the 
MF is an approximately uniform dorsoventral band, not clustered as with the loss of $\mathrm{E}(\mathrm{spl})$, suggesting that Roe is subject to post-translational regulation to achieve such clustered activation (St. Pierre et al., 2002; del Alamo and Mlodzik, 2008). Despite this proposed mechanism, there exists a dearth of evidence in support of the proposed genetic mechanism. The most authoritative study on roe maintains that in mutants, two E(spl) enhancer-reporter constructs, E(spl)m $\gamma$-lacZ and E(spl)m $\beta$-lacZ, both show enhanced activity (del Alamo and Mlodzik, 2008). However, only the $\mathrm{m} \gamma$ report appears to be upregulated in roe mutant tissues, indicating that further genetic analysis is required to further delineate a role for roe in facilitating ato autoregulation (Chapter 2).

\section{Multi-site phosphorylation of M8 provides hints of complex regulation.}

M8 may be subject to multiple phosphorylation events. The CK2 target site of M8 exists within a region of multiple Ser residues that are conserved across Drosophila. Ser ${ }^{151}$ is the modifiable residue within a P-X-S/T-P phosphorylation motif that is commonly recognized by MAPK and Nemo, two kinases that are active within and posterior of the MF, where $m 8^{S 159 D}$ elicits its eye defect (Gonzalez et al., 1991; Clark et al., 1991; Ishitani et al., 2003; Morilla et al., 2012; Choi and Benzer, 1994). Ongoing work is revealing a mechanism wherein phosphorylation of Ser ${ }^{151}$ potentiates $m 8$ as a proneural repressor, though it remains unclear which kinase may be responsible as the Notch pathway interacts with both DER and Nemo during retinogenesis (Bandyopadhyay and Bidwai, unpublished; Baker and Rubin, 1992; Rogge et al., 1995; Verheyen et al., 1996). Interestingly, phosphorylation of Ser ${ }^{159}$, presumably by CK2, may be required to permit modification of Ser ${ }^{151}$, suggesting a multi-kinase procession of stepwise activation. The requirement for a second kinase might explain the temporal gulf in activity that separates $E(s p l) D$ and $m 8^{S 159 D}$, given that $m 8^{S 159 D}$ would likely require further modification at Ser151 to become active whereas $E(s p l)^{D}$ lacks both sites (Kahali et al., 2009). Furthermore, this work places the kinase that acts at Ser ${ }^{151}$ of chief importance in the activation of $m 8$, placing emphasis on the timing of such kinase activity as the "on" switch for proneural repression. Although MAPK becomes active 
concurrently with the onset of stage-3 ato expression, few targets have yet been identified (Chen and Chien, 1999). Furthermore, Sens antagonizes nuclear transduction of the MAPK signal, consistent with reports of a cytosolic hold of activated MAPK (pMAPK) at this time (Pepple et al., 2007; Kumar et al., 1999). Thus, E(spl)D may circumvent the need for activation by pMAPK, a theme that is further explored in Chapter 2.

\section{MAPK relieves Aop-mediated repression.}

Aop is another potential target for pMAPK activity in the developing eye. However, Aop's earliest verified role in eye development is as a negative regulator of R7 specification, as its loss resulted in the formation of supernumerary R7s within mutant ommatidia (Tei et al. 1992). R7 specification had become a focal point of research at the time of this finding in the wake of the discovery that mutation to the RTK Sevenless (sev) results in the complete absence of R7 photoreceptors - a finding that largely shaped early exploration into the interconnection between genetics, signaling and development by indicating that discrete signals transmitted between ommatidial cell types were responsible for the stereotyped formation of the compound eye. In opposition to the loss observed in sev flies, aop mutants elicit an excess of R7s per ommatidium, and loss of aop is dominant to that of sev, demonstrating that aop acts upstream of sev. (Lai and Rubin 1992). These findings spurred further studies, which clarified that phosphorylation of Aop by pMAPK led to derepression of Aop target genes. Although early studies identified nine putative MAPK target sites within Aop, Ser127 is critical to pMAPK-mediated inactivation, as phosphorylation at this site regulates depolymerization, translocation into the cytosol and turnover (Rebay and Rubin, 1995; Zhang et al., 2010). Early studies had merely suggested the possibility that pMAPK regulates Aop turnover. Recent evidence indicates that both Aop and TEL1 monomers are subject to ubquitylation by Drosophila and human F-box E3 ubiquitin ligase Fbl6, respectively (Roukens et al., 2008a; Roukens et al., 2008b). 


\section{Aop interacts with the Notch signaling pathway.}

The aop allelic interactions that had first demonstrated a role in $\mathrm{R} 7$ specification were later found to be sensitive to Notch signaling dosage. Increased Notch dosage, through the use of a genomic duplication, enhanced the supernumerary R7 phenotype, whereas Notch deficiency fully suppressed ectopic R7 formation (Rogge et al., 1995). Counter these findings, later studies revealed a requirement of the Notch signaling component $\mathrm{Su}(\mathrm{H})$ for the expression of Aop (Rohrbaugh et al., 2003). Analysis of a $122 \mathrm{bp}$ enhancer, upstream of the Aop transcriptional start site, houses three $\mathrm{Su}(\mathrm{H})$ binding sites, with two in a paired formation, indicative of a strong binding interaction. Exhaustive enhancer-reporter studies demonstrated the importance of these sites, specifically of the paired sites, to the expression of Aop throughout nearly the entirety of Aop's expression pattern in the Drosophila eye (Ramos et al., 2003). To further intimate a genetic interaction, R7 defects of aop allelic interactions were found to be enhanced in the presence of $S u(H)$ loss of function (Ramos et al., 2003). Additionally, clones mutant for $S u(H)$ were observed as lacking Aop immunostain in the posterior of the larval eye (Rohrbaugh et al., 2003). Furthermore, Notch signaling exerts negative feedback on Aop, as force-expression of E(spl) members M7 and M8 reduces much of the aop enhancer-reporter expression (Rohrbaugh et al., 2003).

\section{Aop regulates eye specification prior to $\mathrm{R} 7$ selection.}

R7 specification is one of the later retinula-directed functions in eye patterning. Aop has also been implicated in patterning events surrounding R8 specification, although reports have widely varied on this claim. Initial reports on aop allelic interactions focused largely on the histological analysis of individual facets, as the unique nature of R7 rhabdomeres allowed for simple quantitation. However, R7 defects, on their own, often fail to elicit major aberrations to the gross morphology of the eye. In addition to R7 defects, aop allelic interactions elicit rough and reduced eyes. While perhaps the roughness can be attributed to the mispackaging of aberrant photoreceptors into ommatidia, the reduction of the adult eyefield is often commensurate with the 
degree of functional loss attributed to each allele, suggesting that Aop might regulate either of two functions - the initial specification of R8s, or later, the maintenance of photoreceptors and associated cells as the fly approaches pupariation (Rogge et al., 1995). Despite the elicitation of a reduction in eye size, our field as a whole fails to recognize that Aop has any autonomous function within cells that are to be specified as photoreceptors during its first stage of expression, as stated in multiple reviews on Drosophila retinal patterning (Kumar 2012, Roignant and Treisman, 2009; Doroquez and Rebay 2006).

Studies regarding Aop function report that Aop is only expressed in and required in undifferentiated basal cells, as a block to differentiation (Rebay and Rubin 1995). However, a report from the same year indicated that in mutant aop cells, neurogenesis failed to initiate, resulting in the absence of all retinulae, including R8s (Rogge et al., 1995). These findings raise the question as to how removal of Aop at its earliest stage of expression could influence R8 formation. If Aop is absent from differentiating cells, such as R8s, then it should only be likely to impact their developmental outcome if it impinges upon some signal being received by the differentiating cells. Thus, it is unclear how Aop functions at this early timepoint of eye development, a theme that is explored in Chapter 3.

\section{Scope of Dissertation}

The following studies aim to better define the nature of ato regulation and the genetic mechanism that governs IG formation. We provide evidence that $\mathrm{E}(\mathrm{spl})$ repressors strictly disrupt ato autoregulation (Chapter 2), that Aop, as a component of the Notch pathway, opposes the effects of E(spl) to facilitate IG formation (Chapter 3), and that Ato proactively facilitates anteroposterior reorganization of the retinal development program through feedback regulation of its enhancers and elicitation of Dpp (Chapter 4). 
A

Notch

Delta

Serrate

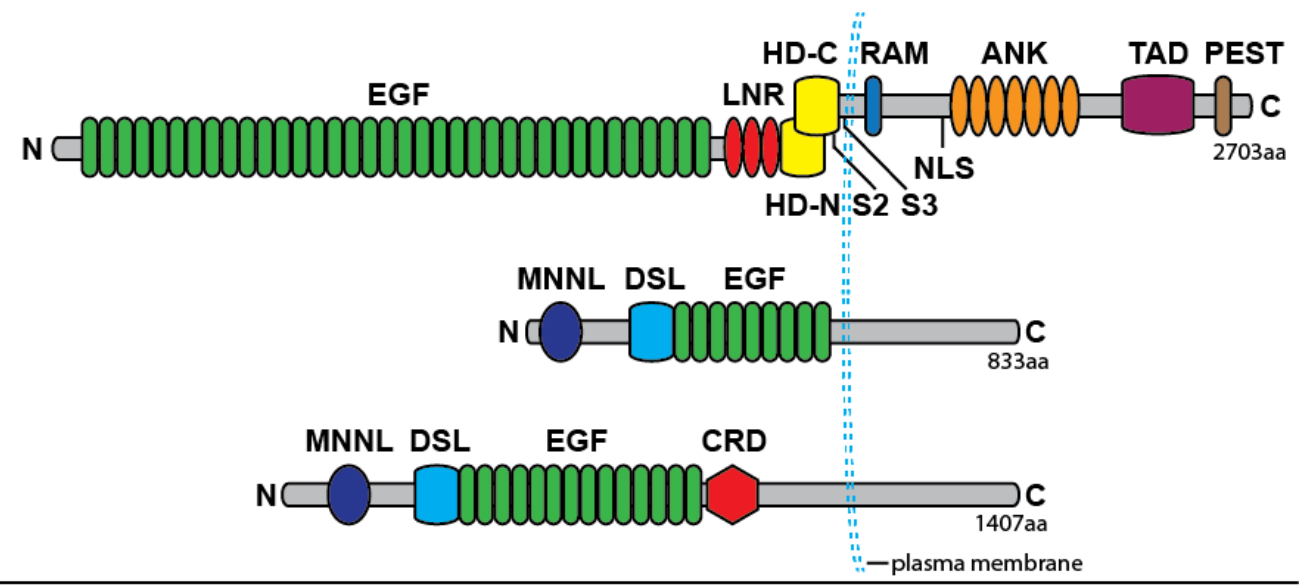

B

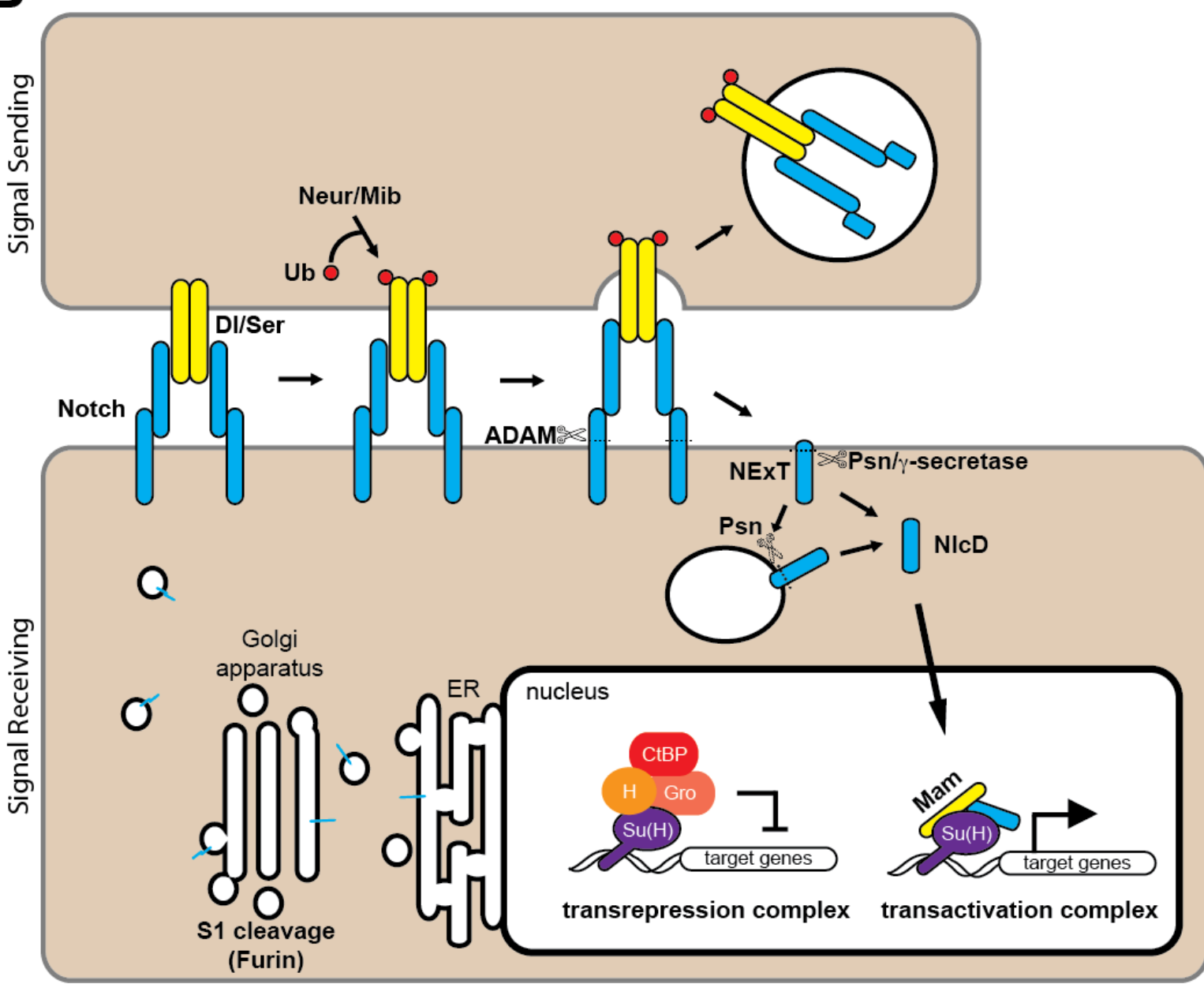

Fig. 1. Notch is a juxtacrine signaling pathway. (A) The domain organization of the mature Notch receptor and its ligands Delta and Serrate. The plasma membrane is shown with extracellular milieu to the left, cytosol to the right. (B) Notch signaling is activated through ligandreceptor interaction between neighboring cells. Ultimately, the intracellular domain (NIcD) of the receptor becomes freed and translocates to the nucleus where it acts as a transcription factor. 
A

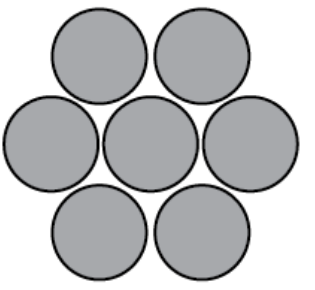

Lateral Inhibition
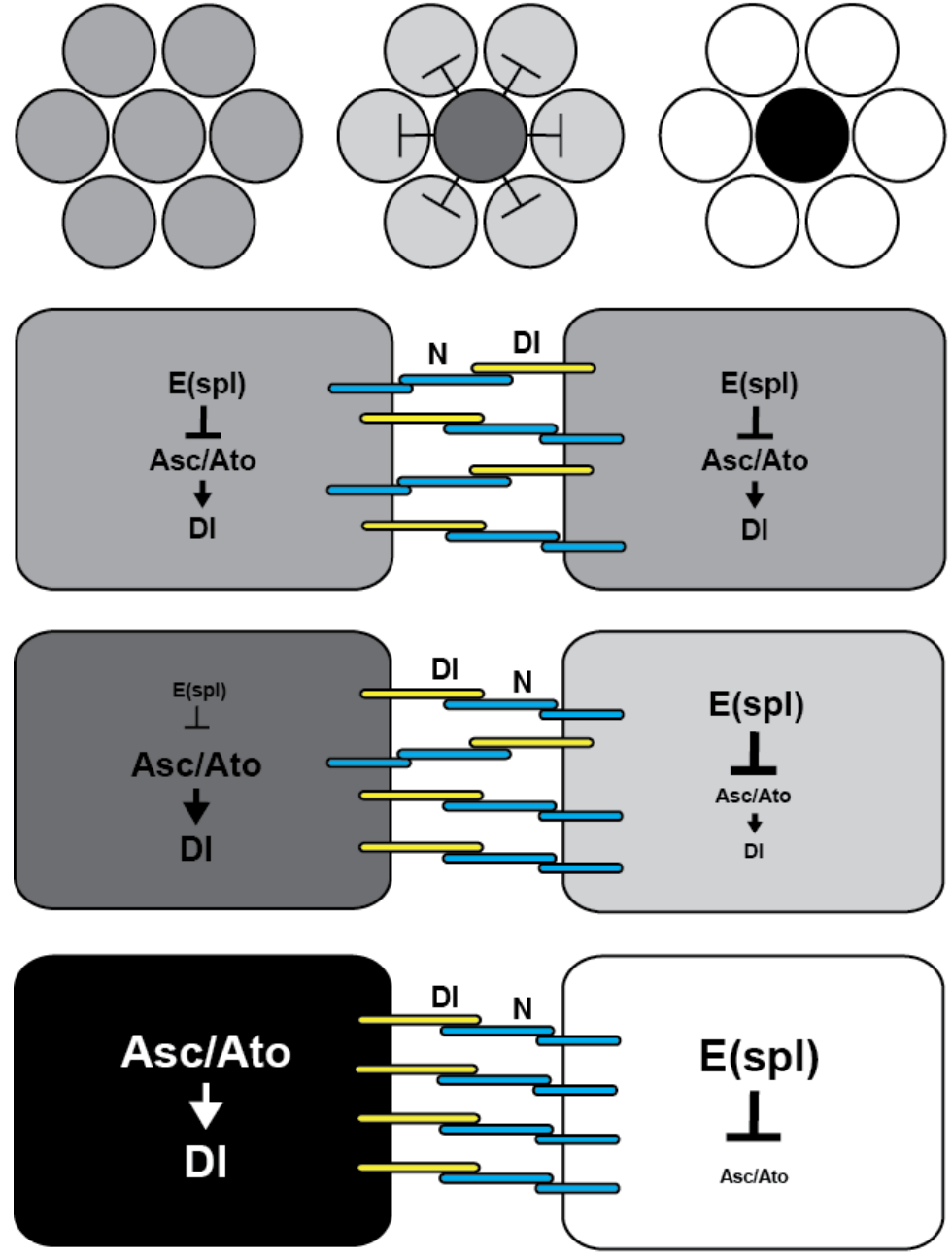

B Assymetric Inheritance
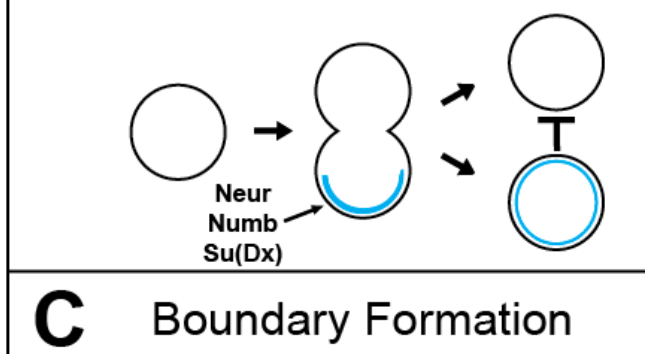

Boundary Formation
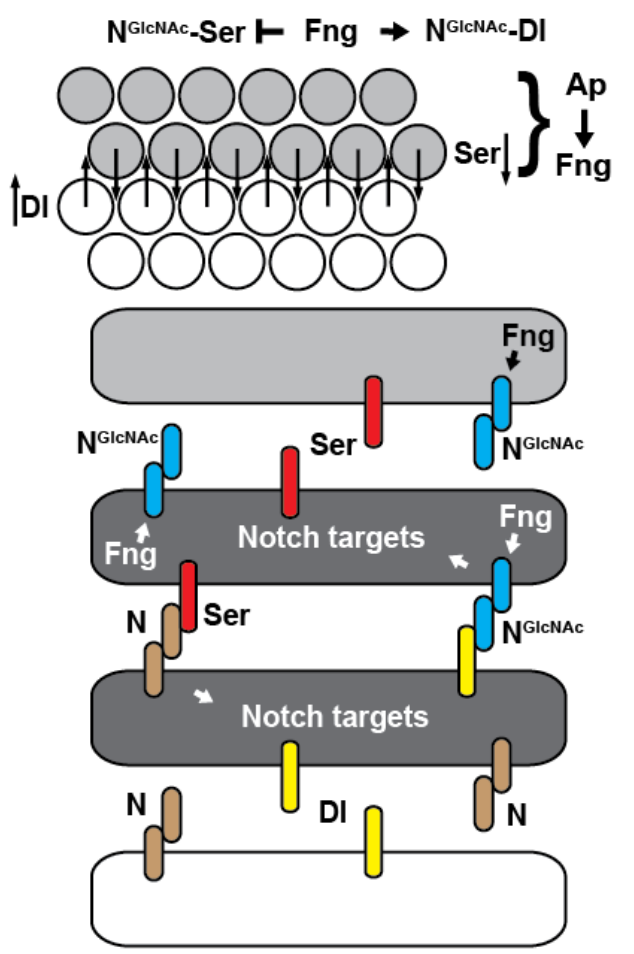

Adapted from Doroquez and Rebay, 2006

Fig. 2. Notch is utilized for three major developmental processes. (A) Lateral inhibition is a process wherein equipotent cells are directed to adopt distinct fates. Equipotent cells "compete" such that the cell that expresses the highest level of proneural also sends the greatest strength of Notch signal to its neighbors. (B) Assymetric inheritance relies upon the unequal distribution of Notch signaling modifiers between daughter cells following cell division. As shown in B, one cell receives greater amounts of Neur, Numb and $\mathrm{Su}(\mathrm{Dx})$, which favor such cell's ability to send a Notch signal while making the cell refractory to receiving the same. (C) Boundary formation can occur where the modification of Notch and its ligands facilitates signaling at only the interface between two compartments. In the dorsal compartment of the developing wing, Apterous (Ap) promotes the expression of Fng. Fng modifies Notch such that preferentially interacts with Dl while disfavoring interaction with Ser. Dl is expressed only in the ventral compartment. Thus, signaling can strongly occur only at the boundary of the dorsal and ventral compartments, where dorsal Notch interacts with ventral Dl, and ventral Notch interacts with dorsal Ser. 


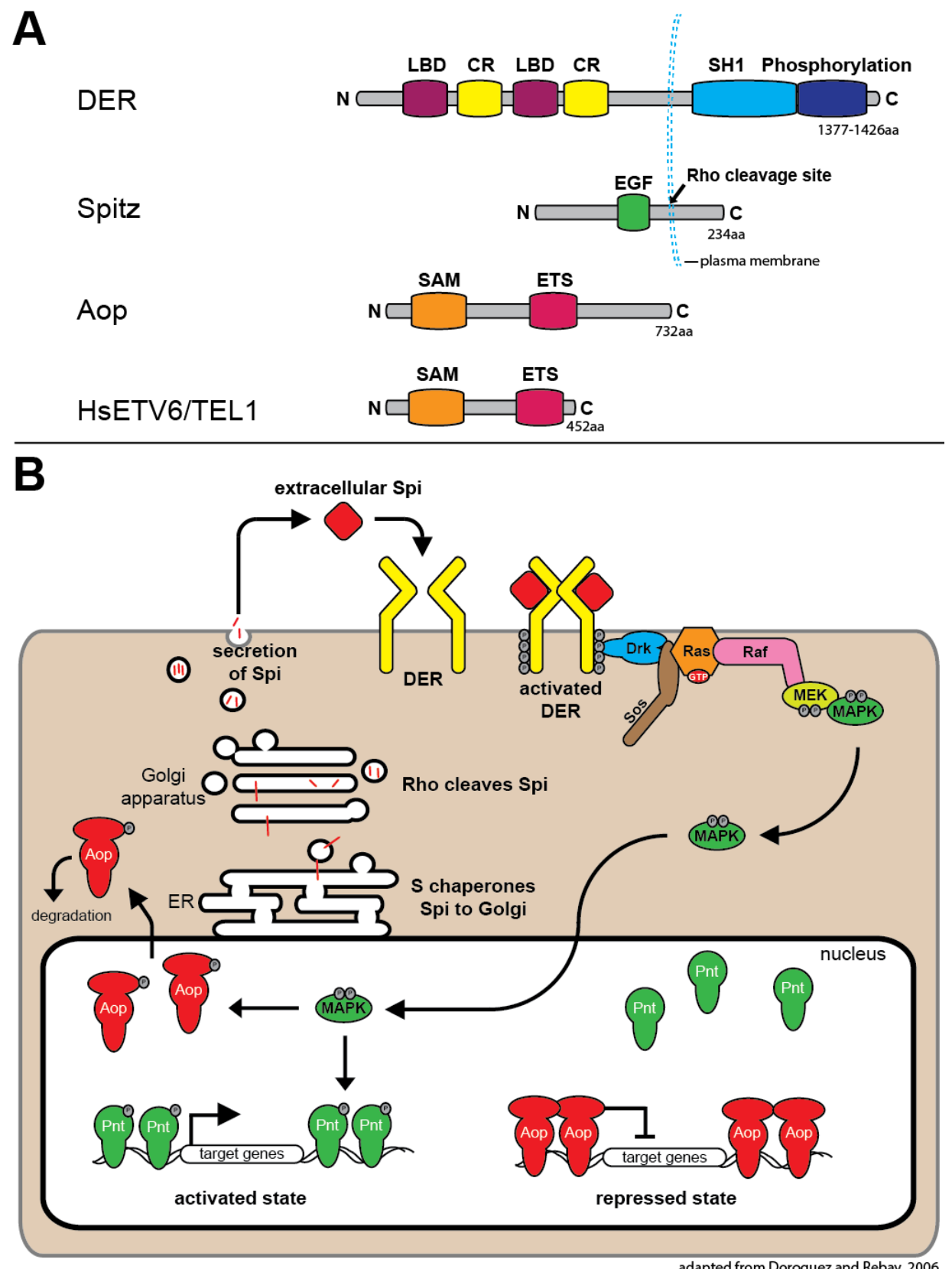

Fig. 3. DER is an autocrine and paracrine RTK signaling pathway. (A) The domain organization of DER, its ligand Spi, and the transcription factors DmAop and HsETV6/TEL1. The plasma membrane is shown with extracellular milieu to the left. (B) Spi is cleaved in the Golgi by Rhofamily proteases, freeing the extracellular domain. Secreted Spi interacts with and activates DER, stimulating transphosphorylation of the receptor pair's intracellular domains. To activate RasMAPK signaling, Drk, Sos, Ras and Raf are sequentially recruited to the cell membrane. Subsequently, Raf phosphoactivates MEK, which in turn phosphoactivates MAPK. Activated MAPK (pMAPK) can undergo nuclear translocation to modify the canonical effectors of Drosophila RasMAPK signaling, Aop and Pnt. Aop and Pnt bind many of the same enhancers. In the absence of pMAPK, Aop binds at multiple sites of target enhancers, repressing gene expression. Upon modification by pMAPK, Aop loses affinity for DNA binding, is exported to the cytosol, and is subject to degradation. pMAPK transforms Pnt into a potent transactivator. 


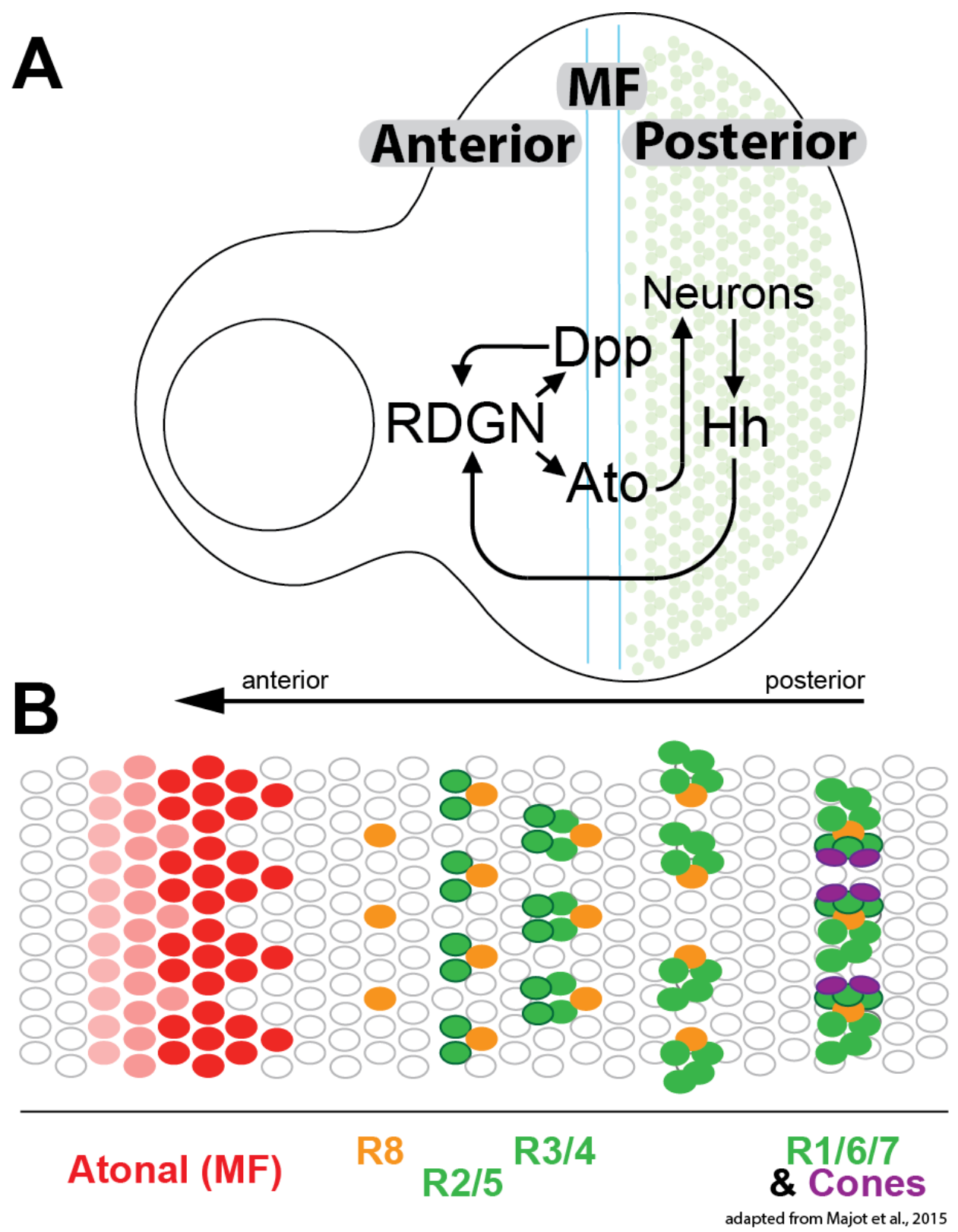

Fig. 4. Comparmentalization of the developing retina. (A) Second/third larval instars feature rapidly growing eye-antennal imaginal discs, antenna portion at left. The eye portion of the disc, at right, is dorsoventrally segmented into distinct anterior and posterior compartments that are separated by the MF. Over the time course two-three days, the MF sweeps anteriorly, expanding the posterior domain at the expense of the anterior. Newly specified neurons of the posterior compartment secrete Hh, which stimulates genes of the RDGN. The RDGN subsequently induces Dpp and Ato within the MF. Dpp is a secreted morphogen that acts most strongly on cells further anterior. These cells modulate RDGN expression, making such cells competent to receive the Hh signal. Ato promotes the production of neurons, providing a genetic cycle that continuously drives the MF toward the anterior. (B) Photoreceptor specificationThe specification initiates following the passage of the MF. Ato is patterned through low-level (pink) and high-level (red) expression domains. Ato facilitates both the patterning and spacing of R8 photoreceptors. Through inductive signaling, the R8 then recruits the R2/5, followed by the R3/4 and later the triad of R1/6/7 (along with the first two cones to be specified). 
A

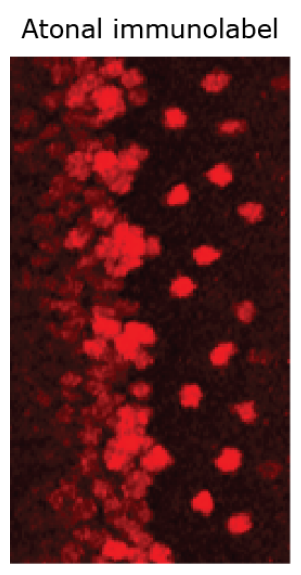

B

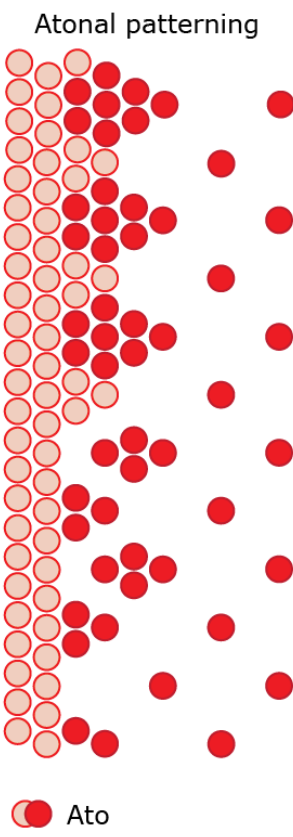

C

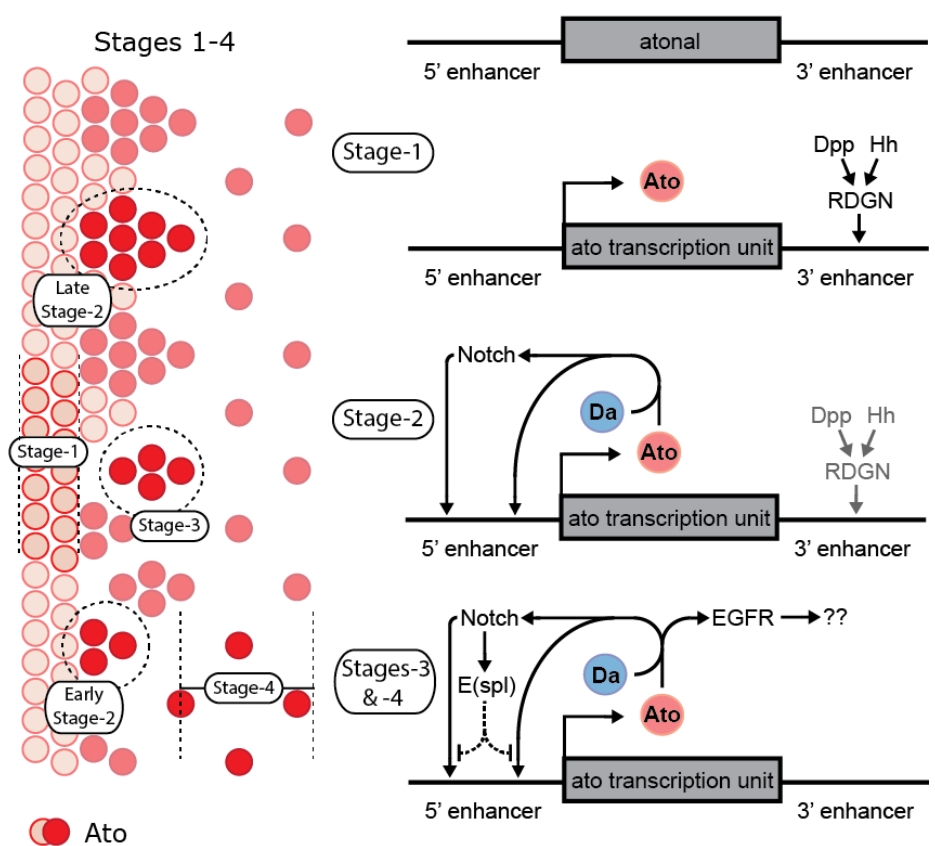

Fig. 5. Ato is dynamically expressed within the MF. (A) An Ato immunolabel indicates a complex expression pattern. (B) A cartoon representation of A; low-level Ato in pink, high-level Ato in red. Ato initiates at low level, in a broad dorsoventral column, stage-1. Stage-2 Ato is marked by progressive upregulation into well-spaced clusters termed IGs. The onset of stage-3 is marked by the repression of Ato, isolating stage- 2 clusters from the main body of stages- 1 and -2 . At stage- 4 , Ato resolves to single cells, R8s, of each cluster, and is maintained for three columns of R8s. (C) ato expression is driven by two enhancers, 5'-ato and ato-3'. Stage- 1 expression occurs as a result of morphogen-dependent RDGN activity directed toward ato-3'. Stage-2 marks the onset of Su(H)independent Notch signaling, which permits Ato and Da to commit to autoregulation of $5^{\prime}$-ato. During stage-2, ato-3' expression becomes lost. Stages-3 and -4 are marked by the induction of $\mathrm{Su}(\mathrm{H})$-dependent E(spl), which disrupts autoregulation. Additionally, though the outcome of such is debated, Ato stimulates DER and MAPK during stages-3 and -4 . 


\section{A E(spl) locus}

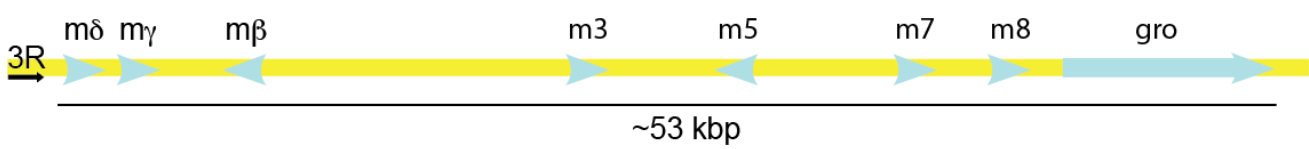

B

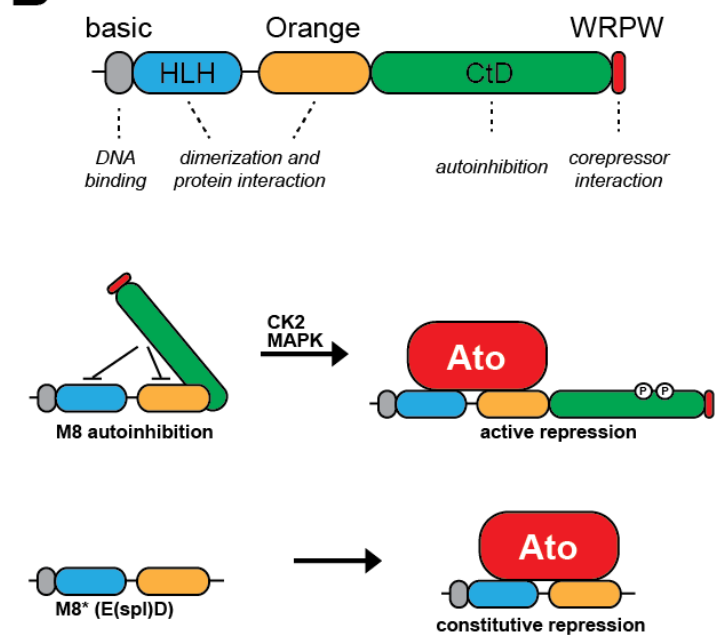

C

Divergent C-terminal domains

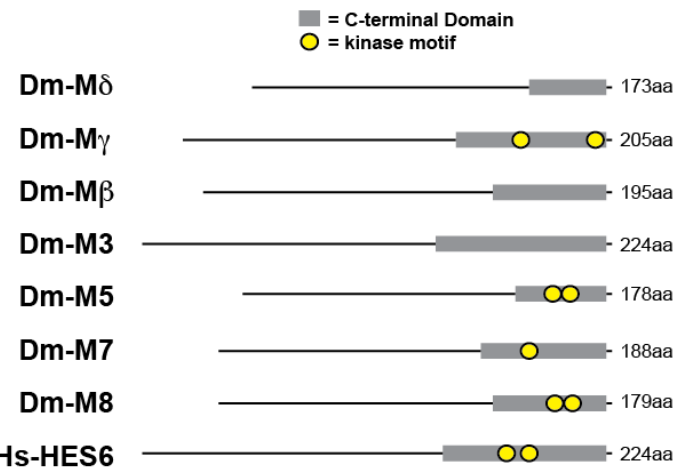

D

Modes of E(spl) repression
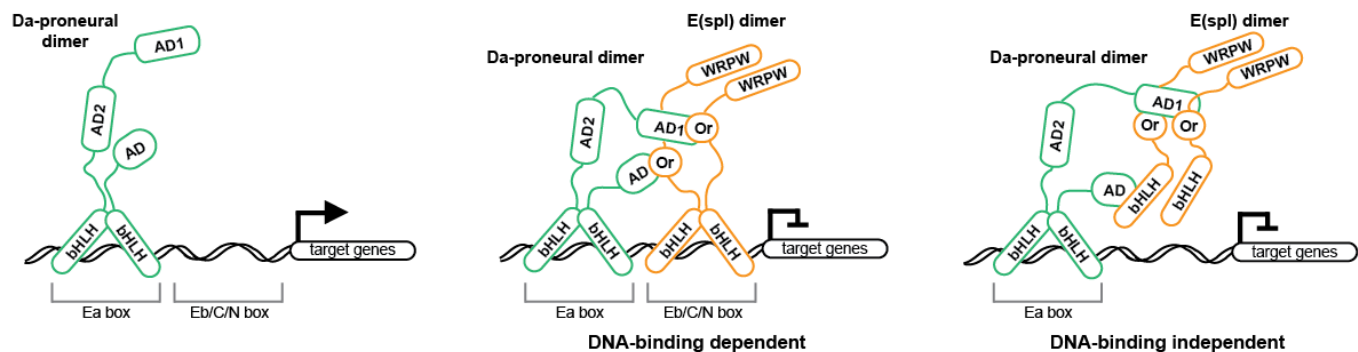

panels adatped from Kahali et al., 2009; Majot et al., 2015; and Delidakis et al., 2014

Fig. 6. E(spl)bHLHs repress proneural function. (A) The $E$ (spl) locus occupies approximately $53 \mathrm{~kb}$ and encodes seven single-exon bHLH repressors and the corepressor Gro. (B) All seven $\mathrm{E}(\mathrm{spl}) \mathrm{bHLH}$ are comprised of the same conserved structure. E(spl)M8 is natively autoinhibited by its CtD. Upon phosphorylation by CK2 and, putatively, MAPK, it becomes an active repressor of Ato, through direct interaction. The $E(\mathrm{spl})^{D}$ mutation encodes a truncated M8 (M8*) that lacks the autoinhibitory domain. Thus, M8* bypasses the need for post-translation activation. (C) $\mathrm{E}$ (spl)bHLHs are of variable length, attributable to divergent CtDs (CtDs in grey). Four of seven $\mathrm{E}(\mathrm{spl})$ members either confirmed or putative phosphorylation motifs (yellow). Confirmation of phosphoregulation in HsHES6 strongly suggests an anciently conserved mechanism for the regulation of E(spl)/HES. (D) Proneural dimers direct gene expression through binding to $E_{a} b o x$ motifs. One mode of $\mathrm{E}(\mathrm{spl})$ repression is for $\mathrm{E}(\mathrm{spl})$ dimers to bind $E_{b} / C / N$ boxes of the same enhancers to which proneurals are bound, occluding proneural transactivation domains and recruiting corepressor. However, DNA-binding is not required as E(spl) are capable of being tethered to target genes through direct interaction with proneural dimers. We propose in chapter 2 that WRPW is not required for repression of Ato by M8. 


\section{Chapter 2: An analysis of transient hypermorphic activity of $E(s p l)^{D}$ during $R 8$ specification.}

\section{ABSTRACT}

Eye-specific expression of atonal (ato) is mediated through dual enhancers: ato-3' regulates initial induction and 5'-ato facilitates Notch-mediated autoregulation. Notch is later needed to resolve Ato to isolated cells from its original broad expression. The molecular mechanisms separating the two, opposing phases of Notch signaling and their relation to ato enhancer activity has remained poorly understood. We demonstrate that Notch-driven E(spl) repressors coexpress in two stages. The first stage of $\mathrm{E}(\mathrm{spl})$ expression is concurrent with Ato prior to the onset of ato autoregulation. $\mathrm{E}(\mathrm{spl})$ is lost from cells that undergo autoregulation, but it returns for a second phase that marks Ato resolution to R8s. Removal of E(spl) prior to autoregulation elicits precocious 5'-ato activity in addition to widespread derepression of the enhancer. Despite encoding a hypermorphic repressor, $E(s p l)^{D}$ does not perturb Ato in otherwise WT flies. However, $E(s p l)^{D}$ interacts strongly with roe, which encodes a repressor that promotes autoregulation through the attenuation Notch signaling. $E(s p l)^{D}$ also elicits Ato patterning defects given reduced dosage of ato and/or sens. These findings indicate that the mechanism of biphasic Notch is not such that repression succeeds induction. Rather, data suggest both pathways initiate simultaneously. Although E(spl) bHLHs as a whole initially delay autoregulation, M8 is held inactive until Ato begins its resolution to R8s. 


\section{INTRODUCTION}

The Drosophila retina is a hexagonal array of approximately 750 ommatidia. Each ommatidium houses eight photoreceptors. The R8 is the first photoreceptor to be specified, to which all other photoreceptors are recruited through inductive signaling. The overall structure of the eye is dependent upon the placement of R8s, which are formed at the lagging edge of the morphogenetic furrow (MF). The MF is a dorsoventral groove in the neuroepithelium that advances from posterior to anterior, starting in the late-second larval instar. R8s are specified through the function of Atonal (Ato), Senseless (Sens) and the Notch pathway(Jarman et al., 1994; Nolo et al., 2000).

Ato expression is dependent on two enhancers located at separate ends of the ato transcription unit (5'-ato and ato-3'; Sun et al., 1998). Ato is first induced in a broad dorsoventral stripe through the action of Hedgehog (Hh) signaling, via ato-3' (Pappu et al., 2005). Once expressed, Ato elicits Notch signaling (Baonza and Freeman, 2001). Notch, in turn, facilitates activation of 5'-ato, which expresses Ato in clusters termed intermediate groups (IGs; Sun et al., 1998). Subsequently, Notch modulates the transcriptional regulator $\mathrm{Su}(\mathrm{H})$ to induce repressors of the $E(s p l)$ locus, which consequently extinguish ato expression (Jennings et al., 1994; Baker et al., 1996). Notch's role in the MF, first as an activator of Ato, and later as a repressor, has been termed biphasic. The mechanism of delay between its two roles has not yet been elucidated.

The $E$ (spl) locus encodes seven bHLH-Orange repressors that bear C-terminal WRPW motifs to facilitate interaction with the corepressor Groucho (Gro, Fisher et al., 1996). Genes of this locus are expressed in various subsets throughout a variety of development contexts (Jennings et al., 1994; Nagel and Preiss, 1999; Cooper et al., 2000; Maeder et al., 2007). Additionally, E(spl) repressors of this locus contain divergent C-terminal regulatory domains with several E(spl) members bearing putative sites for post-translational modification by protein kinases (Trott et al., 
2001; Majot et al., 2015). The most well-studied example of these, $E(\mathrm{spl}) m 8$, referred to henceforth as $m 8$, encodes a phosphorylation consensus motif for the Ser/Thr kinase CK2 (Karandikar et al., 2004). CK2-phosphomimetic M8 has hyperactive repressor activity that, when force-expressed, interrupts Ato and Sens-regulated R8 specification. Thus, in native state, M8-CtD occludes interaction between M8 and Ato, but autoinhibition is relieved upon phosphorylation (Karandikar et al., 2004; Kahali et al., 2009; Kahali et al., 2010). E(spl) ${ }^{D}$, a dominant $m 8$ allele, encodes a truncated protein product, M8*, that lacks the CtD, bypassing the need for phosphoactivation (Welshons, 1956; Nagel et al., 1999). Due to the loss of its C-terminal WRPW motif, E(spl)D cannot bind Gro. Despite this, $E(s p l)^{D}$ elicits retinal patterning defects, though only in combination with the recessive split allele of Notch $\left(N^{s p l}\right) . N^{s p l}$ perturbs eye patterning and causes a reduction in the number of ommatidia (Li et al., 2003).

Attempts to recapitulate the $N s p l ; E(s p l)^{D}$ interaction with the GAL4-UAS binary forceexpression system have revealed somewhat of a paradox. In $N^{s p l}$ flies, force-expression of M8* reproduces the $N^{s p l} ; E(s p l)^{D}$ phenotype, but only when $\mathrm{M}^{*}$ is expressed early in the MF, prior to the onset of 5'-ato activity (Kahali et al., 2009). However, Ato is not repressed in WT flies until later in morphogenesis, after robust 5'-ato activity has been established and at a time where CK2phosphomimetic M8 has been demonstrated to function (Karandikar et al., 2004). This raises two points of interest. First, E(spl) repressors may be expressed earlier in the eye development program than previously considered, and second, the activity of $E(s p l)^{D}$ is strongly affected by the aberration underlying $N$ spl.

To address the first point, evidence suggests that genes of the $E$ (spl) locus are likely coexpressed with Ato in select cells of the MF before E(spl) expression is lost from cells that will form the IG (Baker et al., 1996). The coexpression of a repressor and its target suggests that either the repressor may be inactive for a time or, alternatively, that the gene being repressed has multiple modes of expression and not all modes are simultaneously repressed. 
To address the second point, studies of $N$ spl reveal WT Ato and Sens within the MF.

Patterning aberrations do not become apparent until after the MF, where R8s become lost (Li et al., 2003). Thus, $N^{s p l}$, in isolation, does not readily hinder the earliest stages of retinal patterning.

However, modifiers of $N^{s p l}\left(\right.$ aside from $\left.E(s p l)^{D}\right)$ may impart insight into the nature of $E(s p l)^{D}$ hyperactivity. A screen for modifiers of the eye phenotype of $N^{s p l}$ revealed the zinc finger repressor roughened eye (roe) as a strong enhancer (Brand and Campos-Ortega, 1990). Subsequent analyses indicated Roe to be a positive regulator of 5'-ato (Melicharek et al., 2008; del Alamo and Mlodzik, 2008). Roe expression is stimulated by Notch signaling within the MF and, in turn, Roe binds near $\mathrm{Su}(\mathrm{H})$-responsive enhancer elements that regulate $\mathrm{E}(\mathrm{spl})$ gene expression. Thus, Roe presumably attenuates the expression of $\mathrm{E}(\mathrm{spl})$ repressors in the $\mathrm{MF}$, facilitating retinal patterning (del Alamo and Mlodzik, 2008).

Ato must commit to autoregulation via the $5^{\prime}$-ato enhancer to ensure the robust patterning of R8s. In this work, we have explored a dynamic role for E(spl) in its repression of Ato autoregulation. We provide genetic evidence that $\mathrm{E}(\mathrm{spl})$ repressors antagonize Ato function, both before and after IG formation. Thus, much of IG formation is driven through ato-3' activity, further indicating that the ato-3' enhancer is active until IGs fully mature. Importantly, our analysis reveals that Notch signaling within the MF is less biphasic than it is bimodal, with both the activational and repressive pathways operating in parallel. In the MF, Notch directs simultaneous activation of both 5'-ato and $\mathrm{E}(\mathrm{spl})$ repressors. $\mathrm{E}(\mathrm{spl})$ antagonism of Ato initially prevents 5'-ato activity.

Subsequently, E(spl) is lost from cells to facilitate IG maturation, in a process that requires Roe (del Alamo et al., 2008). Once mature, IGs are subject to a restoration of E(spl). At this time, E(spl) capably represses Ato, which is solely dependent on 5'-ato activity. We propose that phosphoactivation of select $\mathrm{E}(\mathrm{spl})$ repressors functions to allow greater repressive capacity after IG formation, when Ato is expressed at its highest levels in the eye. 


\section{RESULTS}

\section{E(spl) expression evolves with changing Ato pattern.}

Ato facilitates retinogenesis, without which no R8s form (Jarman et al., 1994). The signalregulated patterning of Ato is absolutely critical to the proper arrangement of R8s and the overall eye. As such, the signals responsible for Ato expression have been of premier interest in the study of retinal patterning (Fig. 1). Ato expression is subdivided into four stages. Stage-1 defines the initial induction in broad DV band (Fig. 1C). Stage-2 corresponds to the IG. IGs are comprised of 10-20 cell clusters that are contiguous with stage- 1 and feature high-level Ato expression (Fig. 1C, blue arrow). Stage-3 is reached once IGs become discontiguous from the stage-2 cluster; these cells also label for Sens, which initiates during midway through IG maturation (Fig. 1B-D, blue arrowhead). Stage-4 corresponds to isolated, individual R8s (Fig. 1B-D). In addition to anteroposterior staging of Ato expression, IGs are also phased dorsoventrally. Thus, each IG is dorsoventrally separated by approximately $15 \mathrm{~min}$. (Dokucu et al., 1996). As such, a range of ato stages can be observed within the MF. Fig. 1I-P best illustrates this point; the dorsal half of the MF (Fig. 1I, yellow bar) has a majority stage-3 Ato clusters preceded by stage-1 expression, whereas the ventral half features a majority of stage-2 IGs (Fig. 1I, green bar).

Ato and Notch signaling are co-dependent, thus Ato patterning is best appreciated with respect to the expression of $\mathrm{E}(\mathrm{spl})$ repressors. $\mathrm{mAb323}$, an antibody that recognizes several $\mathrm{E}(\mathrm{spl})$ bHLHs $(\mathrm{m} \delta, \mathrm{m} \beta, \mathrm{m} \gamma, \mathrm{m} 3$ and, to a lesser extent, $\mathrm{m} 8)$, reveals that $\mathrm{E}(\mathrm{spl})$ expression evolves complementary to each stage of Ato patterning (Jennings et al., 1994). At stage-1, E(spl) is absent; an outcome that is unsurprising given the absence of Notch signaling at this time (Fig. 1N, bracket; Baonza and Freeman, 2001; Baker et al., 1996). Stage-2 can be further subdivided into two distinct patterns, early and late. Late stage- 2 Ato is most closely approximated by the stage- 2 patterning already described. Arrows indicate progressively maturing IGs, from dorsal to ventral, to the stage where sens is induced (Fig. 1I-P). Early stage-2 Ato can be differentiated from stage-1 in that at this 
time point Ato only begins to become patterned into clusters and its expression is lesser than that of late stage-2 clusters (Fig 1B, white arrowhead, white arrow). In addition to the contrasted level of Ato expression, early stage-2 Ato colabels with E(spl) (Fig. 1B-H, white arrowhead, white arrow) whereas late stage-2 Ato does not (Fig. 1B-H blue, yellow arrows). E(spl) expression can be observed to the dorsal, ventral and posterior margins of IGs (Fig. 1E-H, blue, yellow arrows). By stage-3, E(spl) fully engulfs Ato-labeled clusters, which are now reduced to fewer cells than seen in stage 2 (Fig. 1E-H, blue arrowhead). Similarly, stage-4 R8s are fully surrounded by E(spl) labeling (Fig. 1I-P, dorsal half). Thus, IG maturation is marked by the progressive loss of E(spl), and R8 resolution is accompanied by the return of $\mathrm{E}(\mathrm{spl})$.

\section{E(spl) and Ato colocalize during early IG formation.}

IG formation is critical to the arrangement of R8s and pattern of the adult retina. Distinct IGs are first visualized during what we have characterized as early stage-2 Ato. Previous efforts using mAb323 and mAb174 (labels only E(spl)m $\delta$ ) demonstrate that E(spl) is coexpresses with Ato to the anterior of IGs (Jennings et al., 1994; Baker et al., 1996). However, technology at the time imposed limitations on the detail of Ato patterning that could be observed. This period of coexpression occurs as Ato is being patterned into early stage-2 IGs. This corresponds to a time when IGs are initially being formed, but occurs prior to their maturation into large clusters that most strongly express Ato (Fig. 1). A combination of in vitro and force-expression evidence suggests that $\mathrm{E}(\mathrm{spl})$ represses Ato function which includes autoregulation (Baker et al., 1996; Ligoxygakis et al., 1998; Lesokhin et al., 1999; Lim et al., 2008). This allows the possibility that E(spl) does not globally repress Ato, as ato-3' is activated independently of Ato and Notch signaling.

In addition to its role in autoregulation, Ato is required for the expression of Sens (Frankfort et al., 2001). Sens promotes the specification and maintenance of the R8 fate (Nolo et al., 2000; Frankfort et al., 2001). Although previous reports indicate that Sens is first expressed within stage3 clusters, our analysis indicates Sens is first induced in mature stage-2 IGs (Frankfort et al., 2001; 
Frankfort and Mardon, 2002; Fig. 1B,D,J,L). Sens labeling is not observed until E(spl) is fully lost from the maturing IG. This suggests that during early IG formation, E(spl) may be antagonizing Ato function (Fig. 1B-H, yellow arrow). Such a role is corroborated by the early upregulation of Sens in $\mathrm{Su}(\mathrm{H})$ mutant clones, from which $\mathrm{E}(\mathrm{spl})$ expression is lost (Li and Baker, 2001).

\section{E(spl) delays activation of the $5^{\prime}$-ato enhancer.}

The precocious induction of Sens that results from the loss of $\mathrm{E}(\mathrm{spl})$ suggests that $\mathrm{E}(\mathrm{spl})$ may actively limit Ato function at this time. As with Sens, 5'-ato also relies upon Ato function for its activity. Thus, we sought to assess the effect that removing $\mathrm{E}(\mathrm{spl})$ function might have on the expression of Ato (Fig. 2). As previously reported, Ato is greatly expanded in retinal tissue lacking the $E$ (spl) locus, using the $E(s p l)$-deficiency $E(s p l)^{b 32.2}$ (Fig. 2A-D; Li and Baker, 2001). To further assess this phenotype, we examined the effect of $\mathrm{E}(\mathrm{spl})$ mutants on reporters for both ato-3' and 5'ato. ato-3' expression appears unaffected in the MF of $E(s p l)$ mutant tissue (Fig. 2E-H). However, $5^{\prime}$-ato is greatly perturbed with respect to its WT report, exhibiting both broader expression that is not confined to IGs and an earlier report (further anterior) than in WT tissue (Fig. 2I-L, yellow arrows indicate mutant tissue, white arrows indicate WT). This result suggests that E(spl) specifically and exclusively represses Ato through 5'-ato, disrupting only autoregulation while leaving 3'-ato as it is.

Additionally, this finding demonstrates that if $\mathrm{E}(\mathrm{spl})$ repressors require post-translational modification to repress Ato, then such modifications are not delayed. Of Drosophila's seven E(spl)

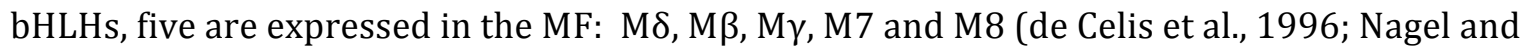
Preiss, 1999). Of these, M $\gamma, M 7$ and M8 are subject to C-terminal phosphorylation by protein kinase $\mathrm{CK2}$; whereas $\mathrm{M} \delta, \mathrm{M} \beta$ lack any apparent motif that is suggestive of $\mathrm{C}$-terminal modification (Jozwick and Bidwai, unpublished; Trott et al., 2001). Our data do not rule out the possibility that some repressors may be constitutively active and immediately repress Ato, whereas others are delayed in their activity. 
To better assess the role of phospho-regulation of $\mathrm{E}(\mathrm{spl})$ repression, we turned to $E(s p l)^{D}$, whose protein product lacks an autoinhibitory domain. The adult eyes of E(spl)D flies are wellpatterned and free of major aberration (Fig. 6A). Having revealed that E(spl) represses 5'-ato, we next tested the effect of $E(s p l)^{D}$ toward the same. Consistent with its WT adult eye phenotype, cells that are homozygous for E(spl)D display no notable change in 5'-ato activity when compared to neighboring heterozygous and WT tissue (Fig. 2M-0).

\section{Hh signaling within the MF is unaffected in E(spl) mutants}

Precocious 5'-ato expression could result from enhanced neurogenesis, as occurs in $E$ (spl) mutant clones (Ligoxygakis et al., 1998). An increased number or density of neurons may elicit heightened expression of the morphogen $\mathrm{Hh}$. Therefore, to rule out possible changes to $\mathrm{Hh}$ response in $E(\mathrm{spl})$ mutant cells, we examined mutant clones for expression of activated Cubitis interruptus (Ciact). $\mathrm{Ci}$ is a transcription factor that progressively accumulates anterior of and within the MF as part of the Hh response (Fig. 2P; Motzny and Holmgren, 1995). Though largely unaffected, $E(\mathrm{spl})$ mutant cells (Fig. 2Q-S, yellow arrows) exhibit a precocious, albeit slight, loss of Ciact as compared to WT tissue (Fig. 2Q-S, white arrows). We hypothesize that this effect might reflect the status of neural hypertrophy in $E(s p l)^{b 32.2}$ clones. Neural specification promotes Ciprocessing through Drosophila EGF receptor (DER) signaling (Baker et al., 2009). Thus, enhanced neurogenesis within $E(s p l)$ mutants is likely responsible for the observed Ciact labeling. Importantly, the reduction in Hh pathway activity in $E(s p l)$ mutants is not likely to cause the observed increase in $5^{\prime}$-ato.

\section{$N^{s p l}$ enhancement by $E(s p l)^{D}$ is only moderately dosage dependent}

$E(s p l)^{D}$ was originally identified as a dominant modifier of the recessive allele $N s p l$

(Welshons, 1956). $N^{s p l}$ flies bear mutant chaetae and, when homo- or hemizygous, severely reduced eyefields with aberrant retinal patterning (Fig. 3B; Cagan and Ready, 1989; Lindsley and Zimm, 1992). To assess both mutations' ( $N^{s p l}$ or $\left.E(s p l)^{D}\right)$ ability to modulate this classical phenotype, we 
have observed the effects of varied dosage. Consistent with a previous report, modulation of the defect, ranked from weakest (most similar to WT) to strongest is as follows: $\mathrm{N}^{s p l} /+<$ $N^{s p l} /+; E(s p l)^{D} /+<N^{s p l} /+; E(s p l)^{D}<\mathrm{N}^{\mathrm{spl}} ; E(s p l)^{D} /+\left(\right.$ Fig. 3A,C-E; Welshons, 1956). As with $N^{s p l}$ males, which are hemizygous, homozygous females exhibit a severe, full reduction of the eye when in combination with only copy of $E(s p l)^{D}$, with few remaining ommatidia (Fig. 3D). However, heterozygous females display a more limited dosage dependence on $E(s p l)^{D}$ (Fig. 3A,C,E). In a $N^{s p l} /+$ background, ommatidial numbers halve upon introduction of one copy of $E(s p l)^{D}\left(N^{s p l} /+\right.$ compared to $N s p l /+; E(s p l)^{D} /+($ Fig. 3A,C). Ommatidial count halves again with the introduction of a second $E(s p l)^{D}$ chromosome (Fig. 3C,E). The genetic relationship between $N^{s p l}$ and $E(s p l)^{D}$ indicates that $N s p l$ contributes more greatly to the $N^{s p l} ; E(s p l)^{D}$ interaction than does $E(s p l)^{D}$. Thus, we reason that further investigation of genetic modifiers of $N^{s p l}$ may better reveal the nature of the $N^{s p l} /+; E(s p l)^{D} /+$, potentially providing insight into the mechanism of $E(s p l)^{D}$ hyperactivity that has, thus far, only been observed in combination with $N$ spl.

\section{$E(s p l)^{D}$ repression is independent of $E(s p l)^{W T}$ dosage}

Our analysis also indicates that $m 8$, the gene primarily affected by the $E(s p l)^{D}$ lesion, is capable of repressing Ato in the absence of Gro binding. Increased $E(s p l)^{D}$ dosage further enhances the $N^{s p l}$ eye defect (Fig. 3C,E). Despite strong repression in $N^{s p l} /+; E(s p l) D$ flies, it is possible that M8* is simply eliciting hyperactivity from other $\mathrm{E}(\mathrm{spl})$ members that maintain their ability to bind Gro.

To assess this possibility, we introduced the $E(s p l)$ deficiency chromosome, $E(s p l)^{b 32.2}$ (with Gro rescue construct) into $N^{s p l} /+; E(s p l)^{D} /+$ flies. Halved dosage of the entire $E(s p l)$ locus elicits no modulation of the eye defect in $N s p l /+; E(s p l)^{D} /+$ flies (Fig. 3C,F). Thus, $E(s p l)^{D}$ is acting independently of other $E$ (spl) genes within the MF. Furthermore, this result indicates that $E$ (spl)D is complete independent of Gro. 


\section{roe interacts with MF mutants}

$N^{s p l}$ must be homozygous or hemizygous to completely ablate ommatidial development when in combination $E(s p l)^{D}$. This indicates that the mutant receptor may be affecting the Notch pathway upstream of $E(\mathrm{spl})$ to create a genetic environment that is sensitized to the specialized nature of $E(s p l)^{D}$. In a screen for modifiers of $N^{s p l}$, roe was identified as a strong enhancer of the $s p l$ eye phenotype (Brand and Campos-Ortega, 1990). roe encodes a $\mathrm{Zn}$ finger repressor that is expressed in the MF (St. Pierre et al., 2002). roe is required for ato autoregulation, as 5'-ato lacks any activity in the absence of roe, though ato-3' is unaffected (Melicharek et al., 2008). Molecularly, Roe functions downstream of Notch signaling to suppress output from $E(s p l)$ genes (del Alamo and Mlodzik, 2008).

To further assess roe's role in the MF, we quantified the ability of roe to modify $N^{s p l}$ and other MF-perturbed mutants (Fig. 4). In agreement with previous reports, $N$ spl interacts strongly with roe, though only when $N^{s p l}$ is homozygous or hemizygous (Fig. 4A-C). DERElp heterozygotes feature a mildly reduced eye with rough patterning (Fig. 4D; Baker and Rubin, 1992). Molecularly, $D E R^{E l p}$ precociously represses ato and this repression is suppressed via reduced Notch signaling (Lesokhin et al., 1999). Ato, as affected by $D E R^{E l p}$, lacks the formation of prominent stage-2 IGs, implying perturbed ato autoregulation (Lesokhin et al., 1999). $R o^{D}$ is a dominant allele of rough (ro), which encodes a homeorepressor that is normally expressed posterior of the MF (Tomlinson et al., 1988; Kimmel et al., 1990; Dokucu et al., 1996; Chanut et al., 2000). $R o^{D}$ eyes feature a distinct anterior cleft referred to as a furrow-stop mutation, which results from failed MF gene expression and a resultant breakdown of morphogen production (Fig. 4G; Heberlein et al., 1993; Chanut et al., 2000). Ro is a repressor of ato (Dokucu et al., 1996). The $R o^{D}$ mutation affects the timing of Ro expression though the protein product remains unchanged (Chanut et al., 2000). Thus, early expression of Ro, from either a heterologous promoter or the $\mathrm{Ro}^{D}$ mutation, elicits Ato defects (Dokucu et al., 1996; Chanut et al., 2000). Thus, we assessed the ability of roe to interact with 
$D E R^{E l p}$ and $R o^{D}$, given their disruption of Ato patterning. In combination with either $D E R E l p$ or $R o^{D}$, roe elicits reduction in the number of ommatidia (Fig. 4D-F, G-I respectively). These data suggest that in each of the backgrounds assayed, roe may enhance deficient ato autoregulation.

\section{roe interacts with $E(s p l)^{D}$}

$N^{s p l}$ is strongly enhanced by both roe and $E(s p l)^{D}$ (Figs. 3,4). Additionally, roe represses E(spl) within the MF (del Alamo and Mlodzik, 2008). Thus, we next assessed whether $E$ (spl) ${ }^{D}$ and roe directly interact. As expected, roe, $E(s p l)^{D}$ transheterozygotes yielded perturbed, slightly reduced adult eyes (Fig. 5A,C). Increasing the dosage of $E(s p l)^{D}$, combining roe heterozygotes with homozygous $E(s p l)^{D}$, strongly enhances the eye defect (Fig. 5A-D). To further test this interaction, $E(s p l)^{D}$ was introduced into a homozygous roe mutant background. A single copy of $E(s p l)^{D}$ strongly enhances the roe mutant eye (Fig. 5E,F). Lastly, we asked whether roe loss of functon could enhance an $E(s p l)$ force-expression phenotype. Force-expression of $m \delta$ with the $h^{H 10}$ GAL4 driver elicits a reduced eye that frequently shows an anterior cleft, reminiscent of the furrow-stop phenotype (Fig. 5G and Ligoxygakis et al, 1998). Reduced roe dosage enhances the eye defect resultant of force-expressed $m \delta$ (Fig. 5G,H). These data are consistent with a mechanism in which roe opposes $E(\mathrm{spl})$ in the MF. Such opposition is very likely borne upon the transcription of $m 8$ as Roe likely regulates $m 8$ in a similar manner as proposed by del Alamo and Mlodzik (2008). Our results further imply that Roe excludes E(spl) from IGs, where Ato commits to autoregulation.

\section{$E(s p l)^{D}$ activity is dependent upon proneural dosage}

Previous attempts to delineate $\mathrm{E}(\mathrm{spl})$ function in isolation of $N^{s p l}$ have made use of forceexpression approaches (Ligoxygakis et al., 1998; Lesokhin et al., 1999; Nagel et al., 1999; Karandikar et al., 2004; Bose et al., 2006; Kahali et al., 2009). However, study of $E(s p l)^{D}$ has remained limited to its effects in the Nspl background (Nagel et al., 1999; Nagel and Preiss, 1999; Kahali et al., 1999). The interaction of $N^{s p l} /+; E(s p l)^{D} /+$ is enhanced by the loss of the proneural genes ato, $d a$ and sens, and the M8 modifier $w d b$ (Nagel and Preiss, 1999; Bose et al., 2014, sens data 
not shown). However, none of these modifiers have been tested for modulation of $E(s p l)^{D}$ in an otherwise $N^{+}$background, partly due to the observation that $E(s p l)^{D}$ homozygotes elicit no major defect in eye size or patterning quality. Furthermore, the research into the aberrant activity of $E(s p l)^{D}$ was rendered null by the tacit possibility that $N^{s p l}$ may simply elicit a Notch signaling response in the presumptive R8. Interestingly, ato loss-of-function, and separately, sens loss-offunction, when transheterozygous with $E(s p l)^{D}$ each resulted in faint ommatidial patterning defects within the mid-posterior region of the eye field (data not shown). To further probe these initial findings, we observed both alleles in combination with homozygous $E(s p l)^{D}$. In both cases, the eye patterning defects became enhanced, decreasing the numbers of ommatidia and perturbing the patterning of the entire eye field (Fig. 6C,D).

The combination of sens and ato mutants with $E(s p l)^{D}$ resulted in a similar exacerbation of patterning defects and ommatidial loss (Fig. 6E). The severity of eye defect was dependent upon $E(s p l)^{D}$ dosage, as $E(s p l)^{D}$, when homozygous in this background, reduced the ommatidial count by over 35\% (Fig. 6F). To indicate whether these defects occur from R8 loss or perhaps some other perturbation, we labeled larval eye discs of sens ato $E(s p l)^{D} / E(s p l)^{D}$ for Ato and the neural marker Elav (Fig. 4C). As with adult eyes, larval retinal patterning was amiss, in places lacking emergent R8s and ommatidia akin to that observed in $N^{s p l}$ (Fig. 6G,H). Older discs exhibit a cascade effect such that as the MF progresses fewer R8s are specified, as indicated by the routine absence of stage-4 Ato (Fig. 6H,H'). This effect may result from the compounding of failed neurogenesis during early retinogenesis. Neuronal loss elicits a corresponding loss of morphogens. The loss of morphogens further decreases the induction of proneural genes within the MF as eye development progresses. In agreement with such a scenario, more mature discs feature a more significant absence of neurons (Fig. 6H, bracket, compared to 6G). 


\section{DISCUSSION}

Ato dynamism ensures that retinogenesis proceeds with the specification of properly spaced R8s. Retinal patterning is dependent upon employment of dual ato enhancers, with the Notch pathway incorporated into the regulation of 5'-ato (Sun et al., 1998; Baker et al., 1996). However, Notch regulates 5'-ato through a bimodal mechanism wherein both $\mathrm{Su}(\mathrm{H})$-independent and $\mathrm{Su}(\mathrm{H})$-dependent responses initiate simultaneously rather than through a staggered response (Ligoxygakis et al., 1998). Previously, the temporal delay between the Su(H)-independent and dependent processes had raised the question of how Notch signaling might function at this time: that perhaps Notch is twice engaged over a short span of time or that or that the $\mathrm{Su}(\mathrm{H})$-dependent response might require that a greater threshold signal intensity be achieved.

Data are not consistent with either previously proposed scenario. As indicated by E(spl) expression data, E(spl) bHLHs can be detected prior to cells' commitment to autoregulation (Figs. 1,7). Loss of $\mathrm{E}(\mathrm{spl})$ at this time results in the precocious activation of 5'-ato and sens, both of which are dependent upon Ato function (Fig. 2; Ligoxygakis et al., 1998). Thus, in WT flies, concurrent use of both modes of Notch signaling (Su(H)-dependent and -independent) allows E(spl) to repress autoregulation (Fig. 7B, early stage-2). During this early stage of E(spl) expression, Ato is solely dependent on ato-3' activity (Fig. 7B, early stage-2). It is not until late stage-2 that autoregulation can set in, coinciding with the loss of E(spl) from IGs (Fig. 7B, late stage-2; Baker et al., 1996). These data obviate the use of signaling threshold activation of the two opposing Notch responses.

With this mechanism as a guide, we next turned to better understand the hypermorphic nature of $E(s p l)^{D}$. As demonstrated, $\mathrm{E}(\mathrm{spl})$ functions during early IG formation to suppress autoregulation. However, M8 is not constitutively active but requires phosphoactivation by CK2 and, putatively, MAPK (Trott et al., 2001; Karandikar et al., 2004; Bandyopadhyay and Bidwai, unpublished). Interestingly, MAPK is active during Ato stages-3 and -4, as Ato is resolved to R8s, but not during early stage-2 (Chen and Chien, 1999). This suggests that M8 is specifically activated 
during stages-3 and -4. Unlike its full-length counterpart, M8* is constitutively active from the loss of its autoinhibitory domain (Karandikar et al., 2004; Kahali et al., 2009; Kahali et al., 2010). Despite this gain-of-function, $E(s p l)^{D}$ elicits no major patterning defect in an otherwise WT background -- not at the level of the adult eye nor during Ato patterning. Thus, altered expression of (as opposed to altered activity of) $E(s p l)^{D}$ is more likely to be the root of its resultant phenotype with $N$ spl.

The interaction between $E(s p l)^{D}$ and roe is evidence that altered expression underlies known $E(s p l)^{D}$ phenotypes. Biochemical and genetic data indicate that Roe is critical to IG formation (St. Pierre et al., 2002; Melicharek et al., 2008; del Alamo and Mlodzik, 2008). Additional, roe mutants enhance the eye phenotype of $N^{s p l}$ (Brand and Campos-Ortega, 1991). Roe permits IG formation by downregulating E(spl) in late IGs (del Alamo and Mlodzik, 2008). Prior to late stage-2, Ato is entirely dependent upon induction from ato-3', making Ato insensitive to repression by E(spl) (Fig. 7). Only once R8 resolution commences does Ato become sensitive to repression by E(spl). This suggests that within fully mature IGs Ato switches dependence from ato-3' to 5'-ato. Thus, Ato becomes strictly dependent upon Roe-dependent autoregulation within mature IGs (Fig. 7B). In WT, $E(s p l)^{D}$ bypasses phosphoactivation with little consequence. However, in roe mutants, $E(s p l)^{D}$ is able to be expressed throughout IGs where it can repress Ato prior to the activation of MAPK.

The eye perturbation of sens ato $E(s p l)^{D}$ flies stands in stark contrast to $E(s p l)^{D}$ interactions with $N^{s p l}$ and roe. As indicated, we reason that $E(s p l)^{D}$ interaction with $N^{s p l}$ and roe are likely the result of $E(s p l)^{D}$ misexpression. In contrast, sens ato $E(s p l)^{D}$ flies are WT for both Notch and roe, allowing us to parse $E(s p l)^{D}$ function from its misexpression phenotype. The sens ato $E(s p l)^{D}$ phenotype cannot be due to repression by M8 during stages-3 and -4, as WT M8 is active at that time as well. Therefore, $E(s p l)^{D}$ hyperactivity is of consequence during early stage-2 in sens ato $E(s p l)^{D}$ flies. Immunolabeling against Ato in such flies indicates that the quality of Ato expression 
and pattern continues to degrade as the MF moves further across the eye (Fig. 6I,J). However, it remains unclear how enhanced $\mathrm{E}(\mathrm{spl})$ repression alone might facilitate the observed phenotype. It is demonstrated that $\mathrm{E}(\mathrm{spl})$ is at this time capable of repressing Ato-dependent activity on $5^{\prime}$-ato and sens. Though purely speculative, it is possible that the level of E(spl) repression during early stage-2 is tuned to permit certain Ato functions while excluding others, and irregularly high-level $\mathrm{E}(\mathrm{spl})$ repression uniformly disrupts all Ato function.

Given that M8 has no apparent requirement for either DNA-binding or Gro-interaction, repression by M8 or M8* requires ratiometric expression commensurate with that of ato. Thus, higher expression of M8* (compared to that of M8) and lack of autoinhibition may combine to artificially increase the ratio of active $\mathrm{E}(\mathrm{spl})$ repressors with respect to Ato in early IG formation (Lai et al., 1998; Nagel et al., 1999). This also hints at why a mix of E(spl) repressors, i.e. those that require posttranslational modification and those that function without, may be employed in the MF. The expression of Ato is markedly lesser anterior to the IG when compared to expression within the IG. Signal-mediated phosphorylation allows for the modulation of E(spl) repressor activity without altering the expression of $\mathrm{E}(\mathrm{spl})$. Ato is expressed at low levels during IG maturation, and at high levels in stages-3 and -4. Thus, the use of M8 allows for CK2 and MAPK to specifically enhance repression only later.

It remains unclear which signals are responsible for regulating the expression and function of roe. Roe expression is promoted by Notch signaling, but low-level expression can be observed in Notch mutant clones when labeled for Roe, indicating that other forces are at play (del Alamo and Mlodzik, 2008). Furthermore, Roe is active only after $E$ (spl) has initially been expressed in the MF (del Alamo and Mlodzik, 2008). It is possible that roe induction may require a higher threshold of Notch signaling. Additionally, DER mutants are highly sensitized to roe mutation (Fig. 4E-G). Functionally, Roe appears to cease its regulation of $E$ (spl) once IGs are fully mature, coincident with the same period that MAPK becomes active. It is plausible that the DER-MAPK signaling axis may 
be required to downregulate roe. The aberrant signaling of $D E R E l p$ mutants can be partially rescued by a reduction in Notch signaling, indicating that cross-regulation of the two pathways may hinge about the regulation of $\mathrm{E}(\mathrm{spl})$. Given that $E(\mathrm{spl})$ are expressed in many MAPK-independent scenarios, including during IG formation, it is therefore more plausible that DER-MAPK might function to sensitize cells of the IG to permit repression of ato by $\mathrm{E}(\mathrm{spl})$.

Ato cannot be repressed without stopping transcription at both enhancers, but E(spl) only targets 5'-ato. The ato-3'-lacZ reporter line used in this work is not suitable for determining when reporter expression ends, as $\beta$-gal is strongly perdurant in Drosophila tissues. RNA in situ labeling of report from ato-3'-lacZ reveals that the 3' enhancer is patterned into IGs, where its expression stops (Sun et al., 1998). Thus, report from ato-3' ceases at the same point of eye development where MAPK becomes strongly active. The possibility that DER-MAPK might negatively regulate ato-3', along with potential effects on the expression and activity of roe have yet to be tested. Our analysis also provides further insight into the mode of repression by M8. Genetic evidence implicates M8* as a hypermorphic repressor (Fig. 3; Nagel et al., 1999). This enhancement exists despite M8*'s inability to bind the corepressor Gro (Nagel et al., 1999; Karandikar et al., 2004). The Achaete-Scute (Asc) family of bHLH proneurals are employed in the specification of sensory organ precursors (SOPs) during Drosophila peripheral neurogenesis. Similar to Ato in eye patterning, Asc are expressed in clusters and resolved to single cells via Notch-mediated expression of E(spl) (reviewed in Furman and Bukharina, 2008). Previous studies indicate that although Gro binding is required for E(spl) repression of Asc, DNA-binding is not (Giagtzoglou et al., 2003). Subsequent analyses in Drosophila and Xenopus indicate that E(spl) and orthologous bHLH-O repressors can forego DNA-binding by directly interacting with their proneural targets (Giagotzoglou et al., 2005; Zarifi et al., 2012). This interaction essentially tethers the repressors to the their targets, facilitating chromatin interaction through the DNA-binding activity of the proneurals being targeted. Repressor-proneural interactions are likely mediated through interaction of the Orange 
domain of bHLH-Os with the transactivation domains of proneurals and their cognate E-proteins (Da in flies; Zarifi et al., 2012). However, if Gro-interaction is required for repression by E(spl), M8* could not directly repress proneurals. E(spl)bHLHs are presumed to function as dimers, though it is not clear whether they might function as homo- or heterodimers; scant interaction data is available (Alifragis et al., 1997; Nagel et al., 1999). Thus, M8* might activate full-length E(spl) repressors through dimerization. Our analysis of the $N^{s p l} ; E(s p l)^{D}$ interaction indicates that this is not the case. $N^{s p l}, E(s p l)^{D}$ transheterozygotes are enhanced by the addition of a second $E(s p l)^{D}$ chromosome (Fig. 3C,E), demonstrating that $E(s p l)^{D}$ does not enhance the activity of full-length M8. $\operatorname{Had} E(s p l)^{D}$ elicited hyperactivity in full-length M8, the replacement of full-length M8 with the second $E(s p l)^{D}$ chromosome would have suppressed the eye phenotype of $N^{s p l}, E(s p l)^{D}$ transheterozygotes. To the contrary, the second copy of $E(s p l)^{D}$ further enhanced the eye perturbations of $N s p l /+; E(s p l)^{D} /+$. To demonstrate that $E(s p l)^{D}$ does not similarly enhance another $\mathrm{E}(\mathrm{spl})$ repressor through heterodimerization, we introduced the deficiency allele $E(s p l)^{b 32.2}$ into $N^{s p l}$, $E(s p l)^{D}$ transheterozygotes. The deficiency allele had little impact on the eye phenotype. This finding demonstrates that $E(s p l)^{D}$ is likely repressing Ato through the direct interaction of $E(s p l)^{D}$ with Ato, and not indirectly through an ability to activate full length repressors that are also present. Thus, $E(s p l)^{D}$ is truly, as Nagel et al. once described, a Gro-independent hypermorph (1999). A Gro-independent mode of repression suggests that the disruption of proneural-mediated transactivation is sufficient to disrupt eye patterning. Additionally, although studies of E(spl) DNAbinding independence were assessed with SOP specification (which utilizes Asc proneurals), it is plausible that E(spl) may target Ato independent of both DNA-binding and Gro-interaction.

\section{MATERIALS AND METHODS}

\section{Drosophila Genetics}

Flies were cultured on yeast-glucose media at $24^{\circ} \mathrm{C}$ and maintained according to a typical diurnal schedule. $D f(3 R) E(s p l)^{b 32.2}$ removes the entire $E(s p l)$ locus including gro (Schrons et al., 
1992). The inclusion of $P\{$ gro +$\}$ rescues cell-autonomous lethality caused by deletion of gro (Heitzler et al., 1996). E(spl)D encodes M8*, an M8 truncation that lacks the CtD (Oellers et al., 1993). $N^{s p l}$ encodes I578T, which alters EGF repeat 14 of the Notch extracellular domain, eliciting altered fucosylation (Li et al., 2003). roe rn16 removes the entire roe coding region (St. Pierre et al., 2002). $r o e^{r n 20}$ removes the entire $r n$ locus, which includes roe (St. Pierre et al., 2002). DerElp encodes A877T, which enhances DER sensitivity to activation (Lesokhin et al., 1999). $R o^{D}$ alters the upstream enhancer of $r o$, eliciting either enhanced or precocious Ro expression (Chanut et al., 2000). $h^{H 10}$ results from a $p G a w B$ insertion in the hairy gene, which is used to drive hairydependent expression of GAL4 anterior to and within the MF (Ellis et al., 1994). UAS-m $\delta$ was made from the insertion of an EcoRI-XhoI fragment of $E$ (spl)m $\delta$ cDNA into pUAST for force-expression (de Celis et al., 1996; Ligoxygakis et al., 1998). sens ${ }^{E 2}$ is a missense mutant that results in premature translational termination (Wang et al., 2010). ato ${ }^{1}$ encodes A25T, K253N, N261I, the last of which ablates DNA binding (Jarman et al., 1994).

\section{Immunohistochemistry}

All steps were performed at room temperature unless otherwise indicated. Tissues were dissected in $0.1 \mathrm{M}$ sodium phosphate buffer and fixed in 4-6\% formaldehyde, $0.1 \mathrm{M}$ sodium phosphate buffer. Tissues were washed in $0.3 \%$ Trition, $0.1 \mathrm{M}$ sodium phosphate buffer bathed in primary antibody mixtures (antibody concentrations shown below in 1\% BSA, 0.1M sodium phosphate buffer) for $12-18$ hours at $4^{\circ} \mathrm{C}$. Primary antibodies were removed, tissues were washed in $1 \%$ BSA in $0.1 \mathrm{M}$ sodium phosphate buffer and bathed in secondary antibody mixtures $11: 1000$ dilution for each secondary, in 1\% BSA, $0.1 \mathrm{M}$ sodium phosphate buffer) for $2 \mathrm{hrs}$. Secondary antibody mixtures were removed, tissues were washed $0.1 \mathrm{M}$ sodium phosphate buffer. Tissues were mounted in 60\% glycerol and imaged using an Olympus Fluoview FV1000 Confocal microscope. All scanning data reported was observed in a minimum of tissues from 5 independent animals of like genotype. 
Primary antibodies include rabbit $\alpha$-Ato (1:5000, Jarman et al., 1994); guinea pig $\alpha$-Sens (1:500-800, Nolo et al., 2000); mouse $\alpha$-E(spl)-mAb323 (1:3, Jennings et al., 1994); mouse $\alpha$ - $\beta$-gal40-1a (1:800-1000, Ghattas et al., 1991); rat $\alpha$-Ciact-2A1 (1:100, Motzny and Holmgren, 1995); rat $\alpha$-Elav-7E8A10 (1:100, 0’Neill et al., 1994). mouse $\alpha$ - $\beta$-gal-40-1a, rat $\alpha$-Ciact-2A1 and rat $\alpha$-Elav7E8A10 were obtained from the Developmental Studies Hybridoma Bank, created by the NICHD of the NIH and maintained at The University of Iowa, Department of Biology, Iowa City, IA 52242. Tissues to be labeled with primary rabbit $\alpha$-Ato were dissected in $0.3 \%$ Triton, 01 .M sodium phosphate buffer.

Secondary antibodies used include 488-goat $\alpha$-mouse (Jackson), 488-rabbit $\alpha$-GFP (Life Technologies), 488-goat $\alpha$-Rat (Life Technologies), 546-goat $\alpha$-rabbit (Life Technologies), 546-goat $\alpha$-mouse (Life Technologies), 546-goat $\alpha$-Rat (Life Technologies), 633-goat $\alpha$-guinea pig (Life Technologies).

\section{Light Micrography}

Adult/pharrate flies were mounted and promptly imaged using a Nikon camera in conjunction with a Leica MZ16 stereomicroscope. For counts listed as "700+", facet count exceeded 700. For the purpose of statistical comparison, individuals were entered into calculations with a facet count of 700 .

\section{Image Production}

All images were processed in Adobe Photoshop CC v. 14.2. Image manipulations of brightness/contrast and color balance were applied uniformly across each image shown. Images were then organized in Adobe Illustrator CC v. 17.1 before being exported to Microsoft Word v. 14.4.4.

\section{ACKNOWLEDGEMENTS}

We are sincerely grateful to Nick Baker, Justin Kumar, Graeme Mardon, Yuh Nung Jan, Hugo Bellen, Sarah Bray and Andrew Dacks, whose gifts of flies and antibodies have enabled this study. 

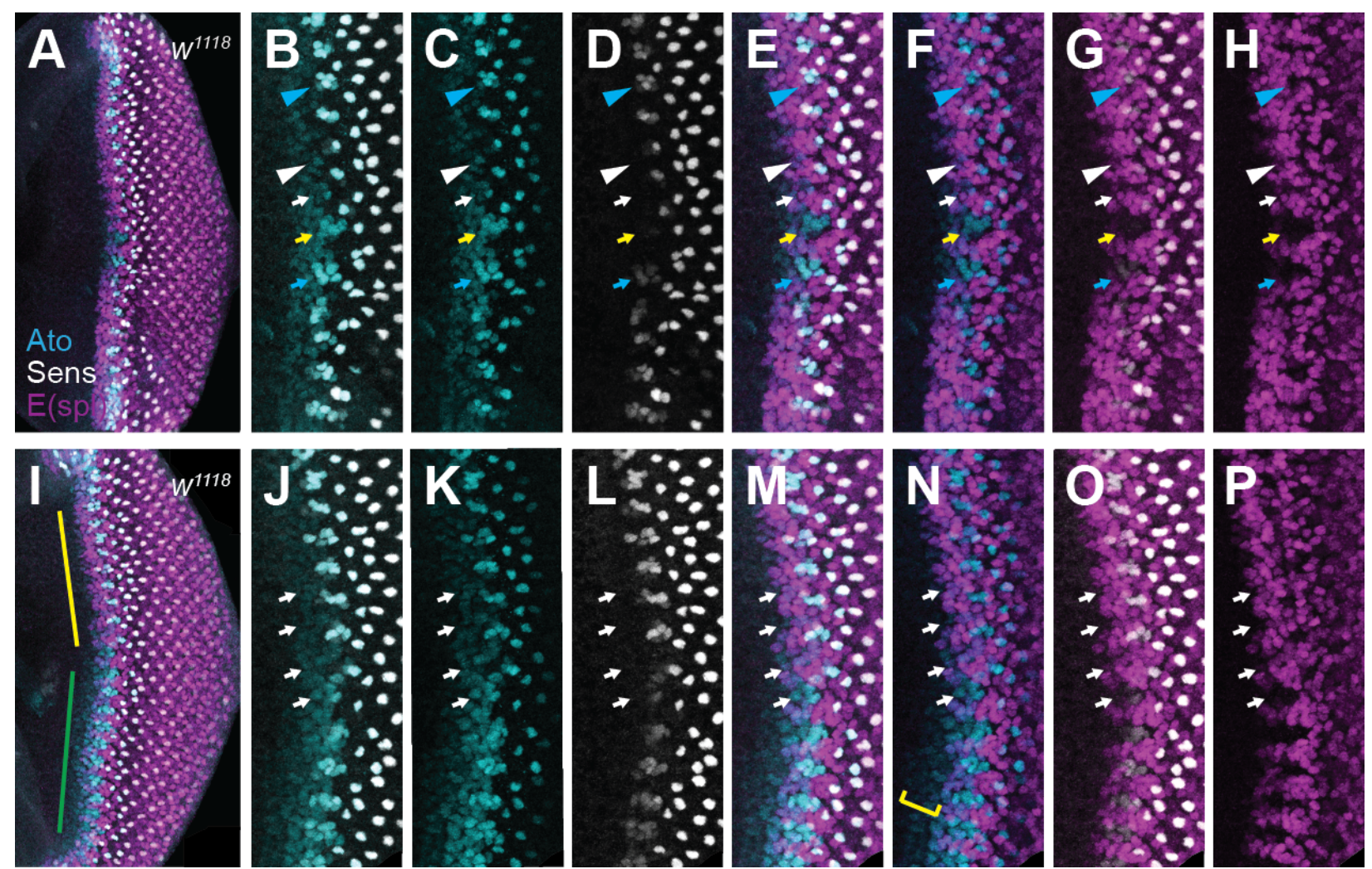

Fig. 1. E(spl) coexpresses with Ato during early IG formation. Panels show WT $\left(w^{1118}\right)$ discs labeled for Ato (cyan), Sens (grey) and E(spl) (magenta), anterior to the left. (A,I) Whole discs and $(\mathbf{E}, \mathbf{M})$ magnification: $\alpha$-Ato, $\alpha$-Sens, $\alpha$-E(spl) staining shows dynamic patterning within the MF. $\mathrm{E}(\mathrm{spl})$ is expressed throughout the MF, at times in a column (I, yellow bar) and other times at evenly spaced intervals (I, green bar). (B,J) Merge of $\alpha$-Ato $(\mathbf{C}, \mathbf{K})$ and $\alpha$-Sens $(\mathbf{D}, \mathbf{L})$. IGs form gradually before the induction of Sens (arrows in B-D). (F,N) $\alpha$-Ato, $\alpha-E(\operatorname{spl}),(\mathbf{G}, \mathbf{0}) \alpha$-Sens, $\alpha$-E(spl), and $(\mathbf{H}, \mathbf{P}) \alpha-\mathrm{E}(\mathrm{spl})$ alone demonstrate that $\mathrm{E}(\mathrm{spl})$ coexpresses with Ato during early IG formation. (M-P) E(spl) is lost from cells as IGs mature (arrows), similar effect observed in (E-H, white arrowhead and arrows). Genotypes: (A-P) $w^{1118}$. 

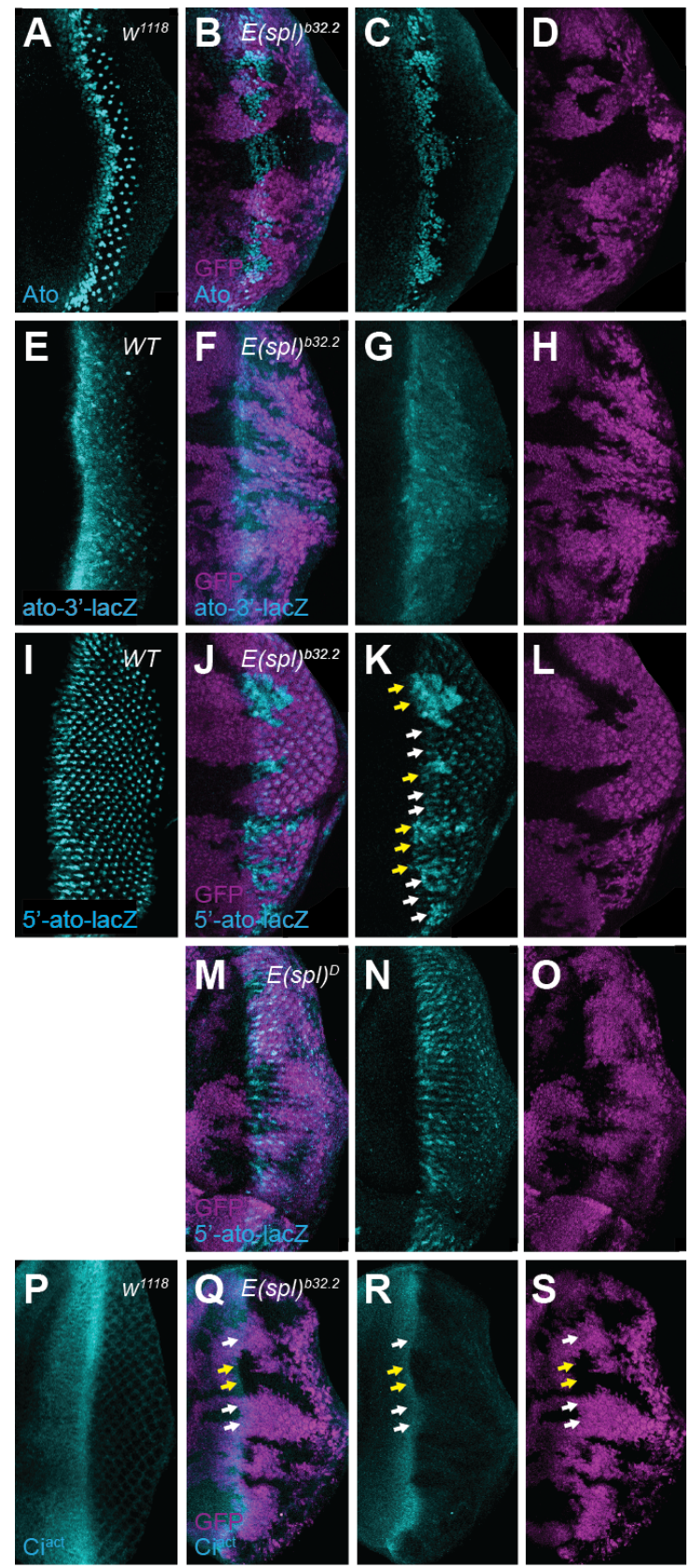

Fig. 2. E(spl) represses Ato autoregulation. (A-D) WT Ato expression resolves to R8s, whereas R8s fail to resolve in E(spl) loss-of-function. As goes for all of fig. 2, the merged panel will be shown first and labeled for the individual channels. Subsequent panels will be each individual channel. Mutant tissues are marked by the absence of GFP (see Methods for mutant generation). (E) ato-3'lacZ enhancer-reporter initiates in the MF and report wanes into the posterior. (F-H) In $E$ (spl) mutants, ato-3' report is unaffected in the MF. (I) 5'-ato-lacZ enhancer-reporter initiates within IGs, resolves to R8s. (J-L) 5'-ato is broadly derepressed and reports precociously in $E$ (spl) mutant cells. (K) Mutant regions are designated by yellow arrows, WT by white arrows. (M-0) $E(s p l) D$ homozygotes have no effect on 5'-ato report when compared to heterozygotes and WT. (P) Ciact progressively accumulates anterior to and within the MF. (Q-S) Ciact is lost slightly earlier in $E$ (spl) mutants (yellow arrows) as compared to WT (white arrows). Genotypes: (A,P) $w^{1118}$, (B-D, F-H, J-L, Q-S) frt82B Df(3R)E(spl)b32.2 $P\left\{g^{2} \mathrm{ro}^{+}\right\} /$frt82B ubiGFP eyFLP, (E) $P\left\{w^{+m c}\right.$ ato3'F:5.8\}/+, (I) $P\left\{w^{+m c}\right.$ ato $\left.5^{\prime} F: 9.3\right\} /+,(\mathrm{M}-0)$ frt82B $e^{*} E(\mathrm{spl})^{D} /$ frt82B ubiGFP eyFLP. 

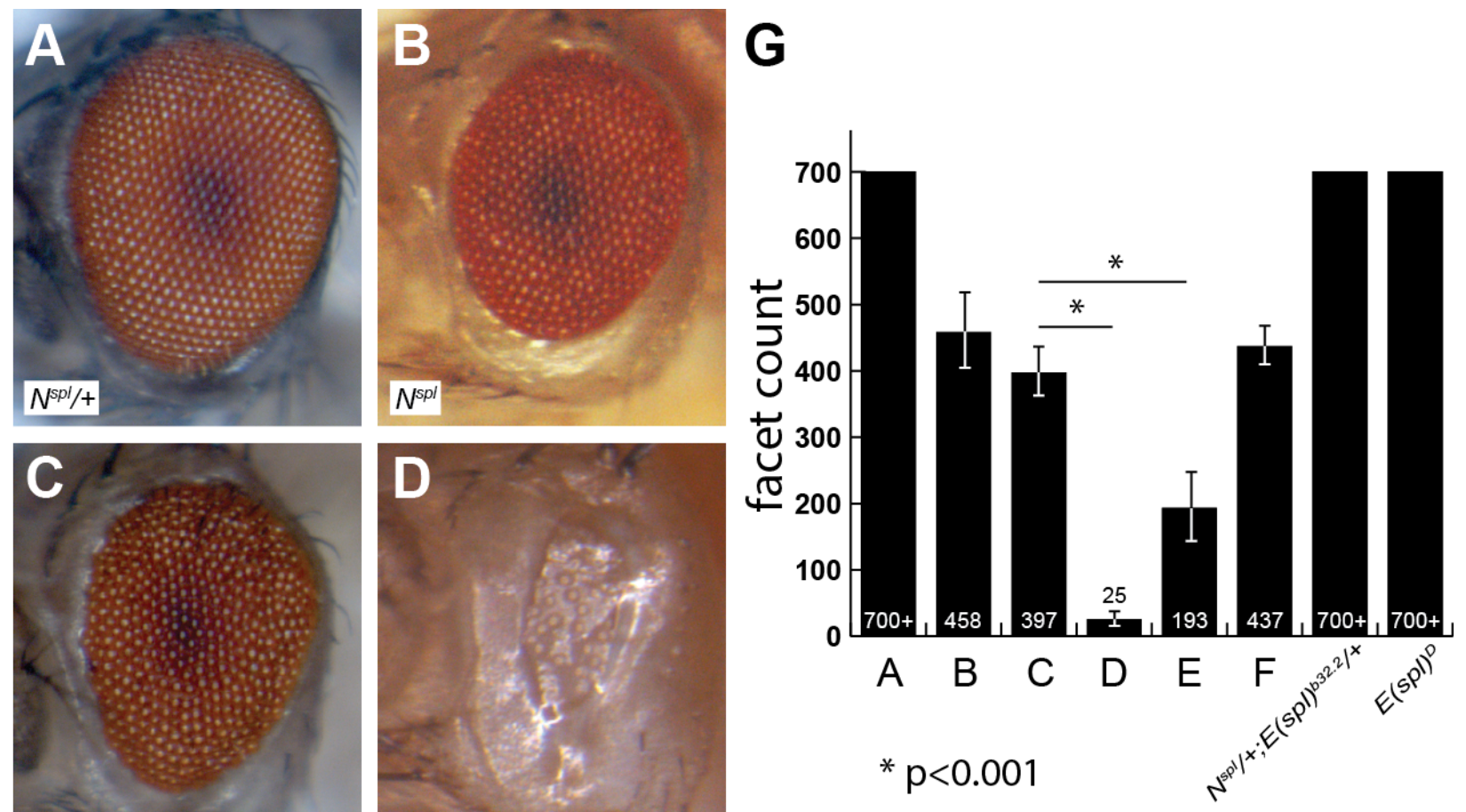

\section{$N^{s p l} /+; E(s p l)^{D /+}$}
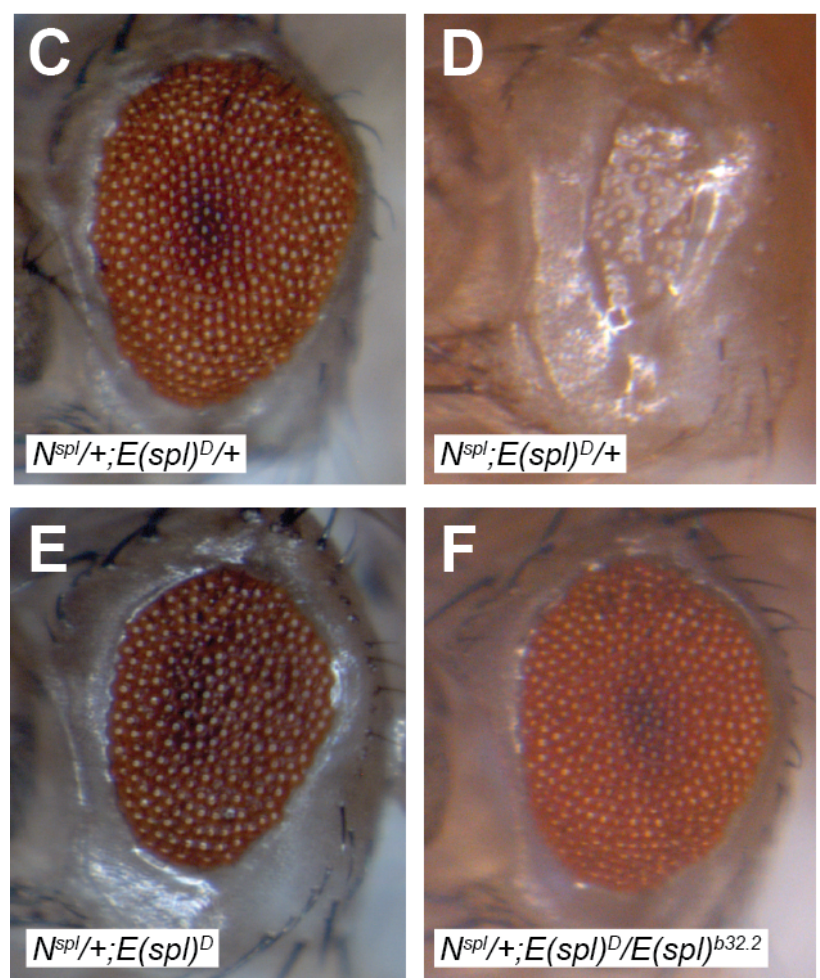

Fig. 3. Dosage contributions to the $\boldsymbol{N}^{s p l} ; \boldsymbol{E}(\boldsymbol{s p l})^{D}$ interaction. (A) $N^{s p l}$ heterozygotes exhibit WT eyes. (B) $N^{s p l}$ homozygotes bear reduced facet counts and atypical patterning. (C) A single copy of $E(s p l)^{D}$ interacts strongly with $N^{s p l}$ heterozygotes, reducing and roughening the eye. (D) A single copy of $E(s p l)^{D}$ nearly fully ablates $N^{s p l}$ homozygote (and hemizygote, not shown) eyes. (E) Two doses of $E(s p l)^{D}$ moderately enhances $N^{s p l}$ heterozygotes. (F) The interaction shown in $\mathrm{C}$ is not modified by reduced $E(\mathrm{spl})$ dosage. (G) Quantitation of phenotypes shown in A-F. $n \geq 10$ for all values shown. Genotypes: as shown, with the exception that full genotype of $E$ (spl) ${ }^{b 32.2}$ is frt82B E(spl)b32.2 P\{gro+ $\}$. 

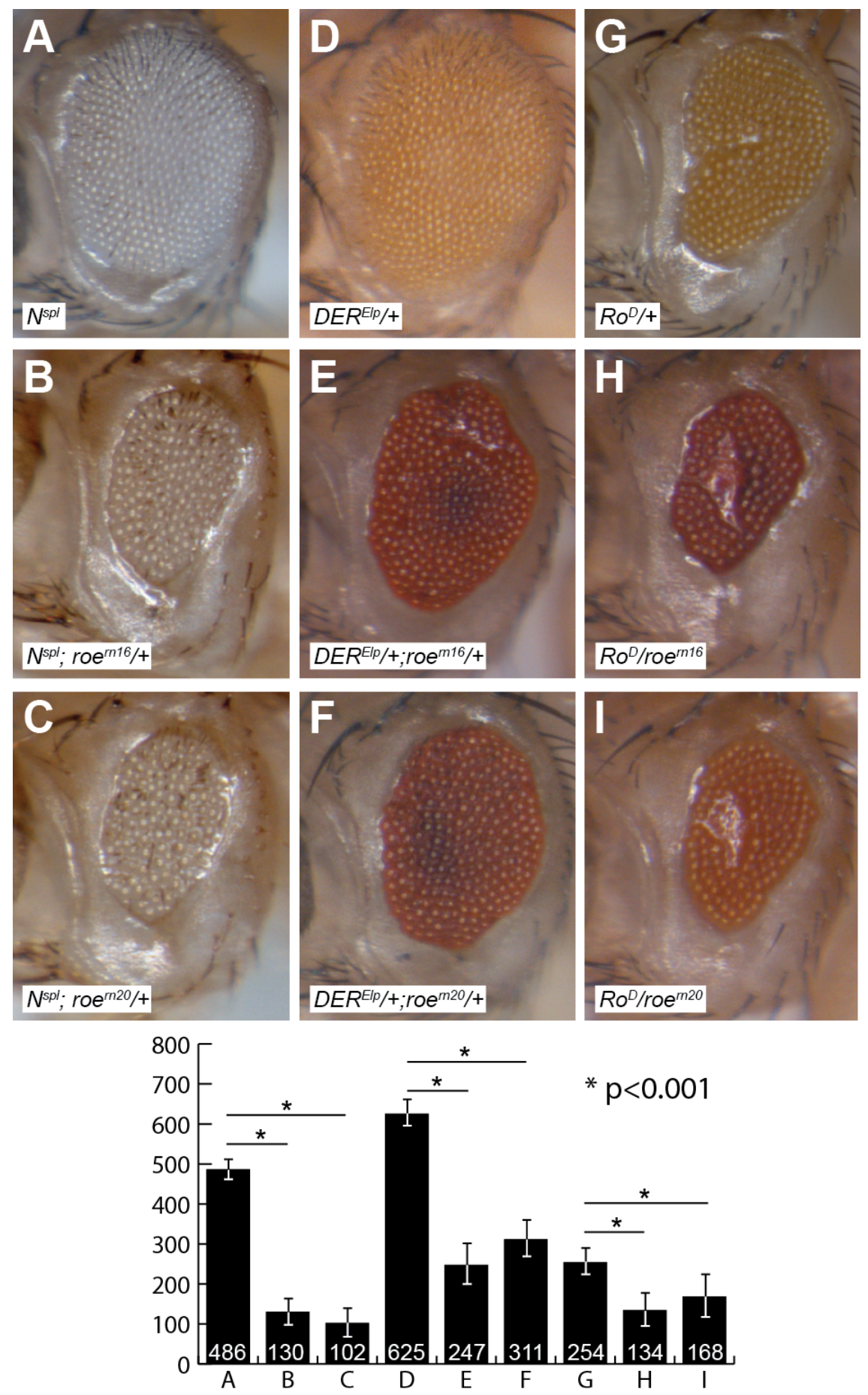

Fig. 4. roe interacts with Ato-sensitized mutants. (A-C) The $N^{s p l}$ eye phenotype is enhanced by roe. (D) $D E R^{E l p} /+$ flies feature mildly reduced, rough eyes. (E,F) roe strongly enhances the $D E R^{E l p}$ eye phenotype. (G-I) $R o^{D}$ flies features a prominent furrow-stop phenotype that is enhanced by roe. Quantitation of phenotypes shown in A-I. $n \geq 10$ for all values shown. Genotypes as shown. 

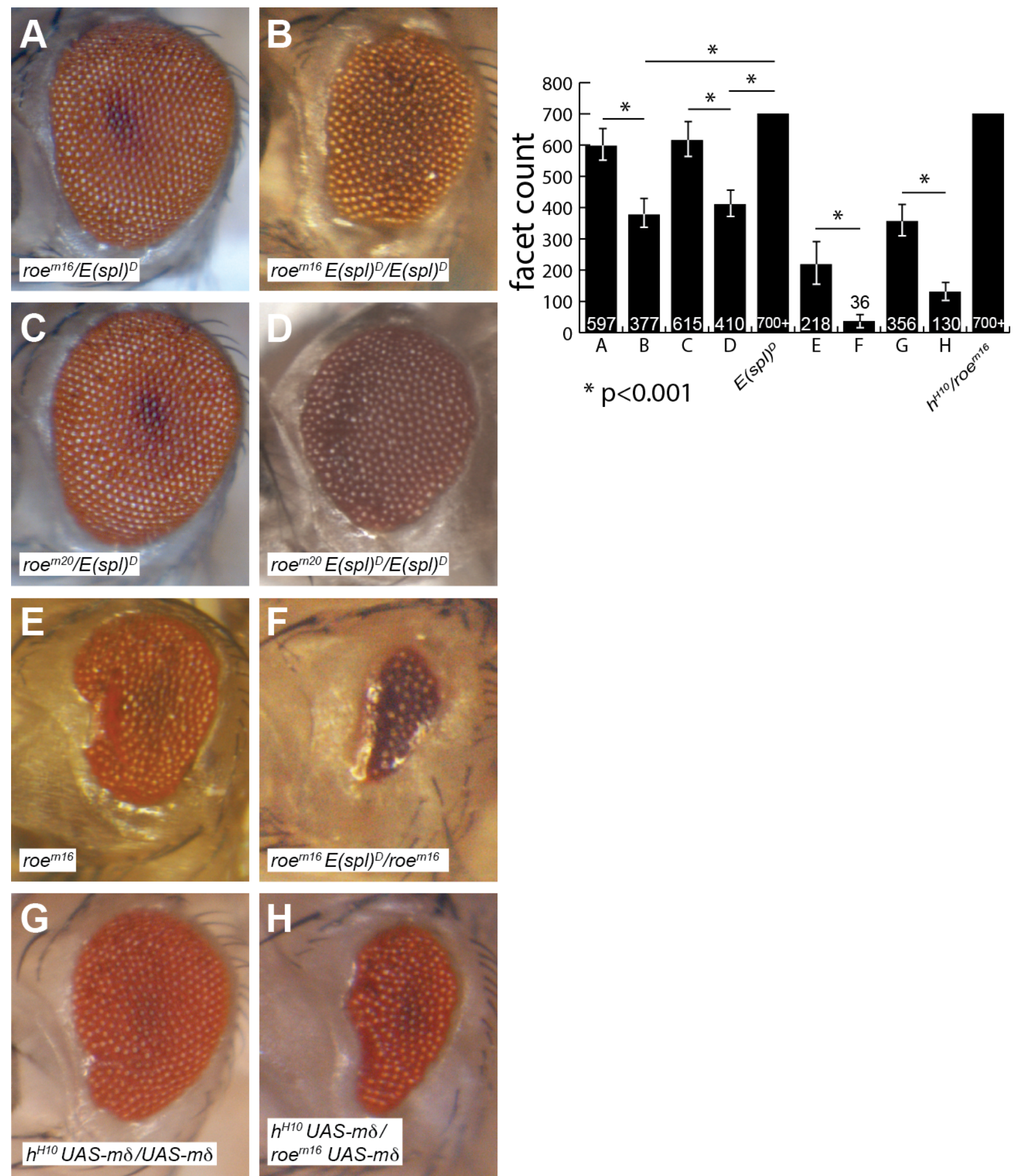

Fig. 5. roe interacts with $\boldsymbol{E}(\mathbf{s p l})^{D}$. (A,C) roe alleles directly interact with $E(s p l)^{D}$, eliciting roughened and reduced eyes. (B-D) The phenotypes of A,C are respectively enhanced by the addition of a second $E(s p l)^{D}$ chromosome. (E) roe homozygotes display severely reduced eyes that often feature an anterior cleft. (F) roe homozygotes are strongly reduced in combination with a single copy of $E(s p l)^{D}$. (G) $h^{H 10} \mathrm{GAL} 4$ driving E(spl)M $\delta$ elicits a reduced eye. roe enhances the $\mathrm{E}(\mathrm{spl}) \mathrm{M} \delta$ force-expression phenotype. (I) Quantitation of phenotypes shown in A-H. $n \geq 10$ for all values shown. Genotypes as shown. 

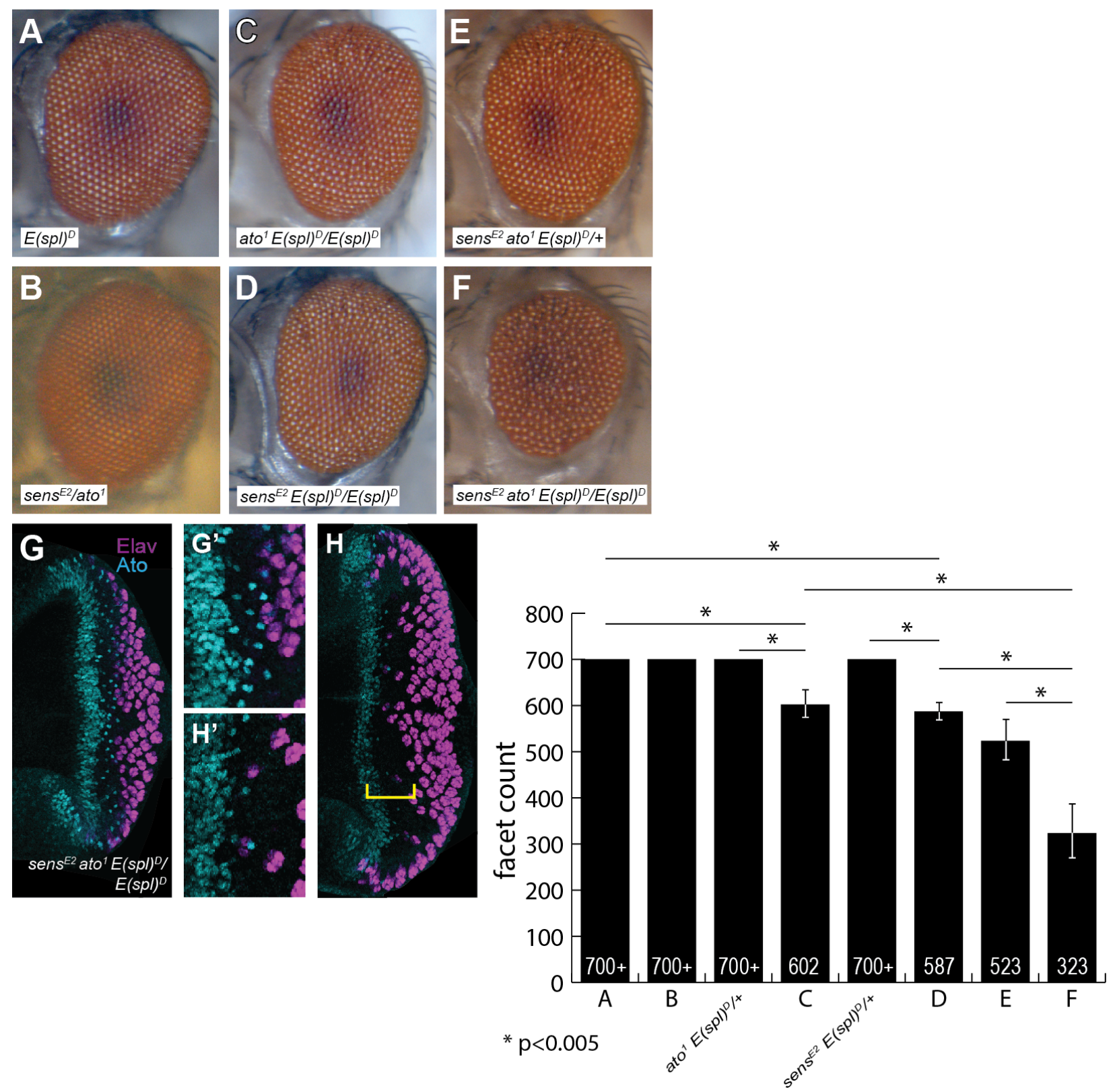

Fig. 6. $E(s p l)^{D}$ perturbs $R 8$ patterning with reduced proneural dosage. $(A, B) E(s p l)^{D}$ and sens/ato eyes are nearly WT in both size and patterning. Both ato and sens, individually in combination with $E(s p l)^{D}$ elicit slight but detectable aberrant eye patterning. (C) $E(s p l)^{D}$ homozygotes in combination with ato results in mildly reduced eyes with perturbed patterning. (D) sens interacts with $E(s p l)^{D}$ homozygotes similarly to ato in C. (E) The combination of sens, ato, and $E(s p l)^{D}$ reduces the eye comparably to the $E(s p l)^{D}$ homozygotes of C, D. (F) sens, ato mutant eyes are further enhanced by two copies of the $E(\mathrm{spl})^{D}$ chromosome. $(\mathbf{G}, \mathbf{H})$ Two sens ato $E(s p l)^{D} / E(s p l)^{D}$ discs stained for Ato (cyan) and the neural marker Elav (magenta). The disc shown in $\mathrm{G}$ is younger; $\mathrm{H}$ is older as the Ato expression domain has progressed further anterior. (G') Magnification of G illustrates the intermittent loss of R8s. ( $\left.\mathbf{H}^{\prime}\right)$ R8 loss in $\mathrm{G}^{\prime}$ is exacerbated as the MF progresses further anterior. Note the loss of nascent ommatidia in $\mathrm{H}$ (bracket) as compared to $\mathrm{G}$. (I) Quantitation of phenotypes shown in A-H. $n \geq 10$ for all values shown. Genotypes: (A-F) as shown. $(\mathrm{G}-\mathrm{H}) \operatorname{sens}^{E 2}$ ato $^{1} E(s p l)^{D} / E(s p l)^{D}$. 
A

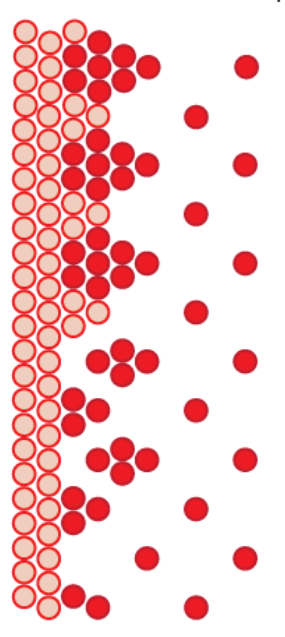

(1) Ato

Ato, $\mathrm{E}(\mathrm{spl})$ Coexpression

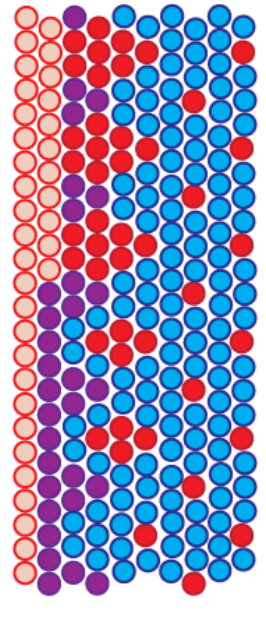

(1) Ato

E(spl)

Ato, $\mathrm{E}(\mathrm{spl})$

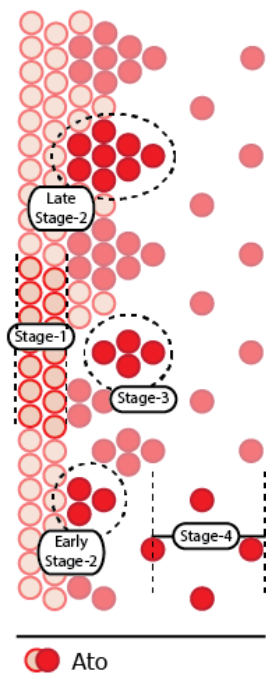

$\mathrm{E}(\mathrm{spl})$ only

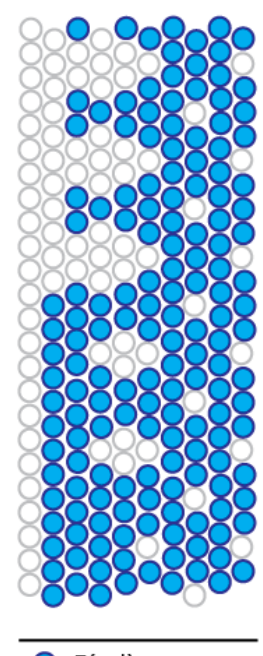

E(spl)

B
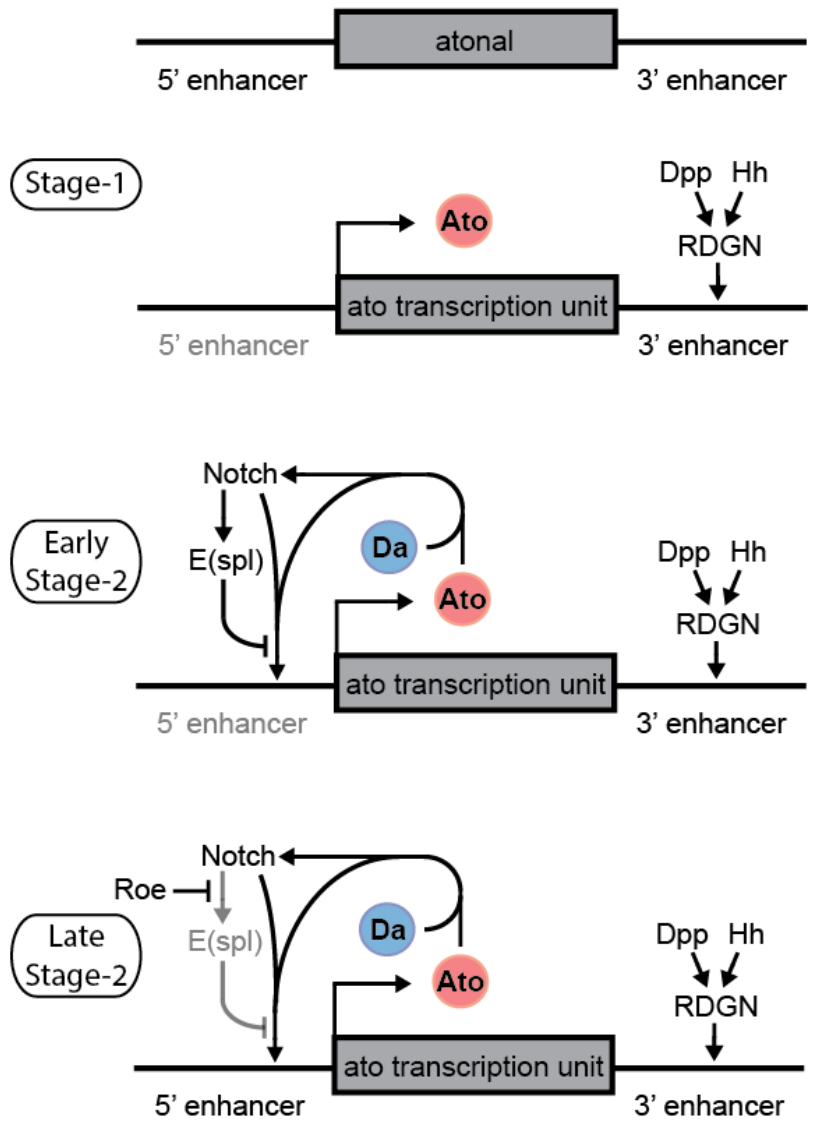

Fig. 7. A mechanism for $\mathbf{E}(\mathbf{s p l})$ repression of ato. (A) Ato patterning is dynamic both anteroposteriorly and dorsoventrally. Ato proceeds through four discrete stages of expression, initiating at low levels during stage-1; evolving gradually through stage-2 to form mature IGs; resolving to R8s during stages-3 and -4. Though E(spl) is excluded from Ato cells during late stage2 through stage-4, E(spl) and Ato coexpress during early stage-2. (B) ato is regulated by two enhancers, 5'-ato and ato-3'. Stage- 1 expression is facilitated by the induction of ato-3' through Dpp and Hh-responsive retinal determination genes (RDGN). Early stage- 2 is characterized by the presence of Ato which, in conjunction with Da, stimulates Notch signaling. Notch, in turn, drives the expression of $\mathrm{E}(\mathrm{spl})$ which then disrupts ato autoregulation. ato-3' remains engaged at this time. Late-stage 2 features Roe, which represses $E(s p l)$, presumably within IGs, allowing the onset of autoregulation via 5'-ato. It remains unclear how soon thereafter activity ceases at ato-3'. Stages-3 and -4 are marked by the return of $\mathrm{E}(\mathrm{spl})$ to progressively more cells of each original IG. ato becomes fully repressed as ato-3' is no longer active. Of note, MAPK becomes active resultant of DER activity (that is driven by Ato and Da). DER activity at this time contributes to the resolution of R8s, though this function is subject to debate. 


\section{Chapter 3: Drosophila aop delays E(spl)-mediated repression of ato.}

\section{ABSTRACT}

Retinal patterning requires the patterning of atonal (ato) through 5' and 3' enhancer modules. ato-3' directs initial expression. Notch exhibits complex regulation of 5'-ato, first facilitating ato autoregulation through this enhancer before subsequently directing repression by E(spl)bHLHs. In this study, we demonstrate that Anterior open (Aop) is required cellautonomously for robust Ato expression during eye development. aop mutants exhibit perturbed Ato expression from both enhancers, showing failed autoregulation and diminished ato-3' report. This abated ato-3' activity is not the result of secondary effects on either Hh or Dpp signaling, but is the result of Aop's co-option by the Notch signaling pathway. Retinal Aop requires Notch signaling as it is expressed downstream of the $\mathrm{Su}(\mathrm{H})$-dependent signal response. During early Ato patterning, Aop is required to maintain ato-3' activity prior to the establishment of autoregulation. Thus, Aop indirectly opposes Ato repression by E(spl)bHLH Notch effectors, as E(spl) genes are both expressed and active in their repression of 5'-ato during this time. In the absence of both Aop and $\mathrm{E}(\mathrm{spl})$, Ato is rescued and R8s, the first photoreceptors that form in eye development, are specified. Furthermore, we have determined that Aop prevents precocious expression of the Ato repressor Ro, but concurrent loss of Aop and Ro has no major effect on Ato loss that is observed in cells mutant for only aop. These findings strongly suggest that Notch signaling, through a potentially conserved relationship with Aop, imposes a delay on Ato repression to facilitate autoregulation. 


\section{INTRODUCTION}

The Drosophila retina is a widely studied genetic model of neuroepithelial organization. In the developing retina, neurogenesis is regulated in time and space through the rate of morphogenetic furrow (MF) movement and the restriction of proneural atonal (ato) expression. The MF, a dorsoventral groove in the retinal anlage, passes from posterior to anterior and is accompanied by neural patterning as regulated by Notch and potentially Ras-MAPK signaling. Within the MF, ato undergoes dynamic expression, wherein it ultimately becomes restricted to precisely-arranged R8 photoreceptors, whose position dictate the patterning of the adult eye. Although ato patterning and R8 resolution are accompanied by Notch and Drosophila Egfr (DER) signaling, no cohesive genetic mechanism has yet emerged to indicate whether DER impinges upon Notch signaling or R8 patterning.

The overall structure of the adult eye depends largely on the mechanisms that regulate ato, which progresses through four stages of expression, initiating anterior of the MF in a broad, dorsoventral stripe (stage-1), before being restricted to clusters of fewer than twenty cells, termed intermediate groups (IGs, stage-2). IGs next become discontiguous from the main body of stage-1 expression (stage-3) before their ultimate resolution of ato to one cell from each original IG, the R8 (stage-4). Stages-1 and -2 require upregulation and occur independently of Suppressor of Hairless $(\mathrm{Su}(\mathrm{H}))$ whereas stages-3 and -4 require the effects of Notch-Su(H)-mediated E(spl) bHLH repressors. Such complexity is achieved through the use of two separable enhancer elements, located at opposite ends of the ato transcription unit and commonly referred to as the 5' and 3' enhancers. ato-3' facilitates stage-1 expression in response to the morphogens Hh and Dpp and their effects on the retinal determination gene network. Stage-2 requires the presence of Ato to induce Notch signaling and enable high-level expression from the 5'-ato enhancer.

The role of Notch has been of great interest in this process, as it is first required for upregulation and later for repression, in a mechanism that has been termed biphasic (Li and Baker, 
2001). To enable ato upregulation, it is proposed that Notch inhibits the bHLH repressor hairy $(h)$ in conjunction with the HLH extramacrochaetae $(e m c)$. With robust ato expression in place, the Notch pathway then turns to favor repression. bHLH repressors of the $E(s p l)$ locus exclusively repress the 5'-ato enhancer (previous chapter). Notably, $E$ (spl) prevent precocious 5'-ato induction, indicating an important role in ato's transition from morphogen dependence to that of autoregulation. as the 3 ' enhancer is required for the incipient stages of IG formation. Autoregulation sets in through the coordination of Roe, a repressor that transiently represses $E$ (spl) to facilitate IG maturation(Chapter 3; St. Pierre et al., 2002; Melicharek et al., 2008; del Alamo et al., 2008). Thus, E(spl) bHLHs actively disrupt autoregulation both to the anterior and posterior of IGs. DER is used reiteratively, if not continuously, throughout eye development, starting with the resolution and differentiation of R8s, the recruitment of outer photoreceptors and the specification of the R7 and cones. In the MF, ato stimulates the production of Rhomboid, an activator of the DER ligand Spitz (Spi, Freeman et al., 1992; Wasserman et al., 2000). In addition to its role in enabling Spi secretion, ato, in conjunction with DER, is required for the cell-autonomous activation of cytosolic MAPK, which undergoes nuclear translocation where it can then modulate the activity of transcription factors such as Anterior open (Aop) and Pointed (Pnt, Chen and Chien, 1999; Lim and Choi, 2004; Baonza et al., 2001; O’Neill et al., 1994). Aop and Pnt frequently act in opposition to each other through the binding of similar enhancers, potentially targeting identical sequence motifs (Xu et al., 2000). In the absence of active, phosphorylated MAPK (pMAPK), Aop represses target genes and Pnt is held inactive. In response to phosphorylation by pMAPK, Aop becomes inactivated and possibly degraded, and Pnt is transformed into a potent transactivator, providing a fast reversal to gene regulatory logic (O’Neill et al., 1994; Rebay and Rubin, 1995).

The use of DER in many diverse roles of eye development is undisputed (Doroquez and Rebay, 2006). However, it is debated whether DER is required for R8 selection, as reports have varied. Numerous studies have demonstrated that DER mutant cells -as well as mutations to DER 
signaling components - result in the formation of supernumerary R8s, as evidenced by the presence of excess Ato-positive cells posterior to the MF, although one report, through use of a temperature sensitive receptor allele, fails to implicate DER in R8 resolution (Lesokhin et al., 1999; Baonza et al., 2001; Lim and Choi, 2004; Rodrigues et al., 2005). Given the discrepancy, it remains unclear whether R8 specification requires DER signaling, though the preponderance of data leans toward some degree of DER-MAPK involvement in the patterning process. Furthermore, MAPK becomes activated in the Drosophila eye with stage-3 ato, where the appearance of pMAPK coincides with the resolution of stage- 3 clusters from stages-1/2 expression (Chen and Chien, 1999). pMAPK persists through stage-4 and is present reiteratively throughout the posterior eye disc compartment where it facilitates later steps in retinogenesis.

Heretofore, no targets of pMAPK have been identified during ato patterning, although both aop and pnt are initially expressed in the developing eye starting with the passage of the MF, where both are dynamically expressed as ato cycles through its four stages (Boisclaire Lachance et al., 2014). Strong aop expression initiates within cells between adjacent IGs and continues posteriorly where aop has been attributed the role of blocking differentiation, preventing retinal cells from responding to low level DER signaling (Rebay and Rubin, 1995). In contrast to this role, the absence of Aop results in an early loss of R8s, as few sens-expressing cells emerge from the MF (Olson et al., 2011). Additionally, Aop may promote R8 specification through its opposition to Wg signaling through direct interaction with the transcription factor Armadillo (Arm), facilitating Arm turnover. Despite support for an Aop-Arm interaction in other developmental contexts, the R8-loss phenotype of aop mutants, was not rescued by concurrent loss of Aop and Wg signaling (Caviglia and Luschnig, 2013; Olson et al., 2011). Also, it remains unclear whether Aop is a target of MAPK within the MF. Genetic evidence indicates that this may be so, as the dominant DER allele Ellipse (Elp) elicits severe eye defects in combination with aop mutant alleles (Rogge et al., 1995). Though the nature of this interaction has not been fully characterized, Elp eyes are partially rescued by a 
reduction of Notch signaling, suggesting that repressive Notch activity may be augmented by DERMAPK ( Lesokhin et al., 1999).

It has been reported that in the developing retina, aop requires $\mathrm{Su}(\mathrm{H})$ for its expression, thus providing a tangible link between Notch and DER signaling axes (Rohrbaugh et al., 2002; Ramos et al., 2003). Here we report that Aop is induced via Notch-Su(H) signaling in the MF where it is required for the maintenance of Ato as IGs mature, in a process that is independent of Pnt. The absence of Aop elicits cell-autonomous loss of stage-2 ato expression. This result indicates that $\mathrm{Su}(\mathrm{H})$, in addition to its defined role in promoting the $\mathrm{E}(\mathrm{spl})$-mediated disruption of ato autoregulation, is also responsible for opposing ato repression through the induction of aop. Such an effect is borne out through ato's dual enhancer regulatory mechanism. During early IG formation, $\mathrm{E}(\mathrm{spl})$ is present to repress $5^{\prime}$-ato. However, Ato is continuously supplied from the morphogen-dependent 3' enhancer, preventing full loss of Ato and R8 fate. In the presence of $\mathrm{E}(\mathrm{spl})$, Aop is able to maintain expression from ato-3' as IG formation proceeds, allowing the onset of ato autoregulation and subsequent processes. Though the precise mechanism of control remains unclear, we have demonstrated that Aop functions within the Notch pathway to impose restraint on neurorepressive $\mathrm{Su}(\mathrm{H})$-signaling, further indicating that DER-MAPK may cooperatively signal with the Notch pathway to serve as a means to delay full implementation of the Notch-repressive program.

\section{RESULTS}

\section{Armadillo is upregulated in neurogenic backgrounds.}

Previous studies suggest that Aop regulates retinogenesis through its physical interaction and destabilization of Armadillo (Arm, ortholog to $\beta$-Catenin) which is enriched in adherens junctions within the MF (Fig. 1A). Accordingly, loss of Aop greatly enhances Arm stability, facilitating aberrant Wg signaling and prolonged apical constriction due to Arm's structural role in adherens junctions. To further explore such a role, we analyzed Arm expression in clones that are 
individually mutant for $a o p, S u(H)$ and $E(s p l)$. If Aop were to maintain neurogenesis via downregulation of Arm in the posterior of the eye disc, we reason that $S u(H)$ mutant tissue, which lacks Aop, should feature both Arm accumulation and a corresponding loss of neurogenesis (Rohrbaugh et al., 2002). As previously reported, Aop clones exhibit high-level expression of adherens junctions-localized Arm (Fig. 1B, arrows). $S u(H)$ mutants feature Arm accumulation that is similar to that in aop mutants (Fig. 1C, arrows). However, in addition to reduced or absent Aop, $\mathrm{Su}(\mathrm{H})$ mutants also feature a pan-neurogenic phenotype unlike that seen with aop such that Elav, which labels neurons, is strongly upregulated across mutants observed within the MF (Ligoxygakis et al., 1998; Olson et al., 2011). This finding is inconsistent with a negative role for Arm during retinogenesis. To further refute Arm as a negative regulator of R8 retinogenesis, we assessed Arm accumulation in $E(s p l)$ mutant tissue, which features a pan-neurogenic phenotype comparable to that of $S u(H) . E(s p l)$ mutant tissue elicits Arm enhancement similar to aop and $S u(H)$ mutant backgrounds (Fig. 1D, between yellow boundaries). Cumulatively, these data suggest that the enhanced apical constriction phenotype is not strictly resultant from loss of Aop, nor is it negatively correlated with neurogenesis. Thus, Aop may regulate retinogenesis through means other than Arm.

Given that Arm appears to have little, if any, direct effect on neurogenesis, we next sought to better characterize the relationship between Aop and the genetic events that occur within the MF. Reports indicate that Aop is required for the production of R8s, as detected by Sens labeling (Olson et al., 2011). Accordingly, R8s were observed, though only in greatly reduced number as compared to WT tissue. In the eye disc, Sens is first expressed in mature IGs and subsequently resolved to R8s (Frankfort et al., 2001). Expression continues within R8s throughout development. In agreement with previous work, Sens-positive individuals are markedly reduced in number within aop mutant tissue (Olson et al., 2011; data not shown). Additionally, clustered IG expression is uniformly lost, with only rare, individualized Sens-labeled cells observed at the normal position of R8s in the 
posterior compartment. This phenotype is consistent with that observed in clones of the aop ${ }^{1}$ mutant, which features a truncated protein product that lacks DNA binding, used throughout this study (data not shown, Caviglia and Luschnig, 2013).

\section{Ato IGs fail to form in the absence of Aop.}

Ato is required for Sens induction. Hence, we sought to assess whether Aop exerts regulatory effects over Ato. Akin to Sens labeling within aop mutants, Ato is aberrantly patterned as compared to WT (Fig. 2; Fig. 3A,C,I for WT). Notably, Ato initiates in mutants as in WT along the anterior margin of its expression domain, presumably via the ato-3' enhancer (Fig. 2A, NOTE the high gain setting to maximize Ato detail). In the absence of Aop, Ato expression fails to progress through its staging, with Ato being lost at the same position where neighboring WT tissues exhibit mature IGs (Fig. 2A, arrowhead). Despite a total failure of IG formation, occasional Ato-positive individuals emerge from the MF (Fig. 2A, arrow). It is probable that such individuals are the same as those few that achieve Sens expression. A second aop mutant clone shows two Ato-positive individuals emerging from the body of Ato labeling, suggesting that the Ato individuals observed result from continuous Ato expression and not from re-induction to the posterior of the MF (Fig. 2B, arrowheads). Notably, the Ato phenotype occurs exclusively in all aop mutant cells, with adjacent WT regions normally expressing Ato, as shown with the IG bisected by a clonal boundary (Fig. 2B, arrow). Thus, Aop cell-autonomously regulates Ato.

To assess whether allele strength of aop might modulate the strength of impact on Ato patterning, we labeled for Ato in clones of the weaker allele aopyan1, which elicits a rough adult eye when homozygous. Unsurprisingly, large clones of aopyan1, when observed across the MF, resulted in decreased intensity of Ato stain and aberrant R8 patterning, including R8 loss (Fig. 2C, arrow). Additionally, some emergent R8s appear to be twinned (Fig. 2C, arrowhead), but are more likely the result of aberrant IG formation wherein only two cells of the precluster indicated have achieved high-level ato expression. 


\section{Ato and Aop colocalize during early IG formation.}

Aop, as with Ato, exhibits staged expression within the MF (Fig. 3). As such, WT Aop expression is best characterized in relation to Ato. During stage- 1 Ato, where patterning events have yet to become apparent, Aop is not present (Fig. 3C, bracket). Aop expression changes rapidly with the onset of IG formation. If we further discriminate the staging of Ato during IG formation, as has been previously shown (Chapter 3), it is possible to classify early, mid and late stage-2 expression. Early stage-2 is revealed as a small cluster of 1-5 cells of slightly elevated Ato (Fig. 3C, yellow arrows), mid stage-2 consists of 10-20 cells of elevated Ato (Fig. 3C, white arrows), and late stage-2 is best characterized by a strong enhancement of Ato expression throughout the cluster, often with highest expression in the furthest posterior cells of the IG (Fig. 3C, green arrow; Fig. 3I, arrows). Aop is either first observed faintly in a uniform dorsoventral stripe where IGs are forming (Fig. 3G, yellow bar), or strongly expressing within cells intercalated between late stage-2 and stage-3 Ato clusters (Fig. 3G, green bar). Within early and mid-IGs, Aop colabels with Ato (Fig. 3D,E, arrows). Once the late-IG is achieved, Aop is no longer found within the IG, but only around its posterior and flanks (Fig. 3H-J, arrows). Aop remains absent from cells that exhibit stage-3 and stage-4 Ato. Additionally, during stage-3, Aop is not restored in cells from where Ato was most recently lost (Fig. 3E,F, arrowhead). This is contrast to labeling for E(spl), which is expressed in all cells surrounding stage-3 Ato (Chapter 3). In a second eye disc, intense Ato labeling similarly reveals that early IGs coexpress Aop. To better view the anterior-most IGs in isolation of the greater Ato expression, only the more basal sections of the tissue can be observed (Fig. 3K-M). High-level Ato coexpresses with Aop within the anterior-most IGs(Fig. 3K-M, white arrows) but not those that are more mature, to the posterior (Fig. 3K-M, yellow arrows).

\section{Aop cell autonomously regulates Ato.}

Ato's dependence on Aop is dictated cell-autonomously given that the high-level Ato expression sharply follows clonal boundaries (Fig. 2B). Thus, Aop is directly impinging upon the 
the nuclear dynamics that allow for continued Ato expression as opposed to a secondary, indirect effect such as may be mediated through the elicitation of extracellular signaling. Importantly, Ato and Aop are only coexpressed in early- and mid-IGs. Thus, Aop is responsible for the maintenance of Ato throughout this short window of time during IG formation. The incipient moments of IG maturation are driven primarily through ato-3', as E(spl) is present and active in its repression of the 5'-ato.

\section{Aop imposes upon both ato eye enhancers.}

Ato is regulated via two enhancers during eye development; ato-3' permits initial Ato expression whereas 5'-ato is autoregulatory. In WT, 5'-ato is strongly visible in all R8s throughout the posterior compartment and in larger clusters near the MF, a likely reflection of expanded expression within IGs (Fig. 4A). We assessed enhancer activity in aop $1 /$ aopyan1 transheterozygotes, a background that elicits greatly reduced adult eyes and aberrant ommatidial patterning (Rogge et al., 1995). In the mutant background, both Ato and Sens patterning are perturbed - Ato achieves few IGs, though the IGs that do form are strongly Sens-positive (Fig. 4B,D). Despite Sens defects within the MF, many R8s emerge from the MF, though patterning is amiss with intermittent loss of Sens labeling. Aop is also involved in many later steps of eye development that are likely the result of the aop $1 /$ aopyan 1 adult phenotype. 5'-ato also reports in the developing antennae, which remains unaffected in aop mutants (data not shown).

Expression of the $5^{\prime}$-ato enhancer is potentiated by a form of autoregulation wherein Ato is required, either directly or indirectly to stimulate the enhancer (Sun et al., 1998). The earliest stage of Ato expression is resultant of ato-3' enhancer activity. Therefore, a loss of 5'-ato induction could be elicited by a reduction in ato-3' activity. The 3' enhancer provides a strong dorsoventral band of expression that initiates at the MF in WT, and wanes toward the posterior of the eyefield (Fig. 4C). In an aop mutant background, ato-3' is severely reduced with the detection of only basal expression, and none of the high-intensity report near the MF that is characteristic of WT (Fig. 4D). 
Thus, the combination of enhancer results suggest that the Aop is responsible for the maintenance of ato-3' activity within early to mid-IGs and that the loss of 3' activity likely diminishes autoregulation. Further evidence that Aop affects the 3' enhancer can be found in the previous clonal analysis with Ato labeling. In aop mutant clones, Ato fails to pattern into any rudimentary form that is recognizable as an IG. The early stages of IG formation are dictated by ato-3', indicating that the perturbation to ato-3' underscores the loss of 5'-ato activity.

\section{MF progression is not affected by aop.}

3'-ato enhancer activity is first induced anterior to the MF as the culmination of retinal determination gene network activity. The RDGN transcription factors Ey, So, Eya, and Dac all bind the 3' enhancer to stimulate transcription of ato (Zhang et al., 2006; Tanaka-Matakatsu and Du, 2008; Tanaka-Matakatsu et al., 2014). Therefore, the RDGN transcription factors are an essential cog in the cycling of $\mathrm{Hh}$ and Dpp, the two morphogens that cooperatively drive the MF toward the anterior of the eyefield (Borod and Heberlein, 1998). Dpp is secreted from the MF and acts in the anterior compartment of the developing eye to oppose Wg signaling (Bessa et al., 2002). As a result, RDGN activity increases, promoting Hh sensitivity and neural competency (Greenwood and Struhl, 1999). Hh is secreted from differentiation, non-R7/8 photoreceptors (Lee et al., 1992; Heberlein et al., 1993; Ma et al., 1993). Hh response, as monitored by the accumulation of the activated, full length form of Cubitus interruptus (Ciact), increases throughout the anterior compartment of the eye as the MF nears before quickly dropping off in response to neuronal differentiation and DER signaling (Fig. 5A; Baker et al., 2009).

A loss of Ato via the 3' enhancer may result from either a loss of secreted Dpp or perturbation in the Hh response. However, aop mutants have no apparent effect on the Hh response, as mutant clones exhibited a WT accumulation of Ciact as the MF approached, and a subsequent dropoff in accumulation as cells entered into the posterior compartment (Fig. 5B, arrows). Likwise, Dpp expression, as indicated by a lacZ reporter, showed continued Dpp 
expression as compared to WT (Fig. 5C,D, arrows), suggesting that Dpp production is sufficient in mutant tissue (Blackman et al., 1991). Asterisk in Fig. 5D indicates a fold at the ventral margin of the tissue and is not resultant of mutant activity.

\section{pnt mutation does not impair Ato.}

In the Drosophila DER-MAPK pathway, Pnt opposes Aop upon MAPK activation (O’Neill et

al., 1994). Thus, our investigation necessitates assessing the role of Pnt in the patterning of Ato and the patterning of morphogens. Previous studies have indicated that Pnt, downstream of DER, is required for the acquisition of non-R8 neural fate, as well as the degradation of Ci from neurons of the posterior eye compartment (Yang and Baker, 2003; Baker et al., 2009). Our analysis shows that Pnt does not perturb Ato patterning (Fig. 5E), despite the failure of Sens to resolve in pnt mutants (Yang and Baker, 2003). Although Pnt modulates both Hh secretion and response, we find that it has no effect on the expression of a $d p p$-lacZ reporter (Fig. 5F, arrows; Rogers et al., 2005; Baker et al., 2009).

Lastly, to assess whether concurrent loss of Aop coupled with reduced Pnt could rescue the Arm phenotype of Fig. 1, we constructed aop and pht dual-mutant clones. Dually mutant tissue failed to rescue the enhanced apical constriction phenotype observed in only aop mutant tissue (Fig. 5G, arrows). Together, these data suggest that Pnt serves no role in regulating apical constriction.

\section{Aop is a component of Notch signaling throughout eye development.}

Having determined that Aop is required for the maintenance and maturation of IGs, but not for the progression of Hh-Dpp cycling, we next sought to further define the role of Aop within the context of Notch signaling in the MF. Aop expression in the eye superficially resembles that of other known $\mathrm{Su}(\mathrm{H})$-dependent genes, with dynamic staging that is similar to the expression of $\mathrm{Su}(\mathrm{H})$ dependent E(spl) (Lecourtois and Schweisguth, 1995; Baker et al., 1996;; Chapter 3). Previous studies indicate that the aop locus includes an upstream enhancer that is responsible for eye 
expression and bears high-affinity $\mathrm{Su}(\mathrm{H})$ paired sites (Ramos et al., 2003). Biochemical analysis indicates that $\mathrm{Su}(\mathrm{H})$ binds to the paired sites and clonal analysis provides evidence that in the absence of $\mathrm{Su}(\mathrm{H})$, Aop is not expressed in the posterior eye disc (Rohrbaugh et al., 2002).

To further this analysis, we assessed Aop expression in ato and Notch mutant backgrounds and within larger clones of $S u(H)$ mutant than previously reported, and within $S u(H)$ clones that span the MF (Fig. 6). Aop is expressed in ato mutant tissue, though its expression is delayed (Fig. 6A), displaying similarity to the expression of $\mathrm{E}(\mathrm{spl})$ in da mutant clones (Lim et al., 2008). In Notch mutants, Aop fails to express in either the MF or to its posterior (Fig. 6B). Unsurprisingly, $\mathrm{Su}(\mathrm{H})$ mutant tissue lacks Aop labeling throughout the developing eye, at both the MF and all positions posterior (Fig. 6C). However, this finding is at first paradoxical in that we also report that loss of Aop alone results in failed IG formation - a result that is juxtaposed against the well-established point that $\mathrm{Su}(\mathrm{H})$ loss of function permits large-scale upregulation of Ato, Sens and the neural marker Elav. Previous analyses have concluded that $\mathrm{Su}(\mathrm{H})$-mediates the expression of $E$ (spl), which are responsible for restricting Ato, Sens, and neurogenesis to a select few cells (Lai and Rubin, 2001). Our investigations into this subject demonstrate that $\mathrm{E}(\mathrm{spl})$ targets repression of Ato through only the 5' enhancer (Chapter 2). Thus we conclude that despite the loss of Aop from within $\mathrm{Su}(\mathrm{H})$ mutant tissue, the loss of $\mathrm{E}(\mathrm{spl})$ and consequent upregulation of the 5 ' enhancer is dominant to the effect had by mutant aop on ato-3'.

\section{Aop as a positive regulator of ato.}

Aop has a well-documented role as a transrepressor. Therefore, in order for Aop to transactivate or maintain ato, Aop likely represses a repressor of ato. There are several known candidates: the homeoboxes Bar and Rough (Ro) and the bHLH-O genes of the Enhancer of split (E(spl)) complex. We reason that Bar and Ro are the most promising candidates given that Aop promotes ato-3' activity, whereas $\mathrm{E}(\mathrm{spl})$ regulates ato exclusively through the 5 ' enhancer. Lastly, it 
is possible that Aop might be indirectly modulating expression of known positive regulators of ato. Thus, we also assayed for Aop regulation of the zinc finger Roughened eye (Roe).

\section{Aop prevents precocious Ro expression.}

The Ro homeorepressor is expressed in the posterior compartment of the developing eye, in a narrow dorsoventral band of cells posteriorly adjacent and complementary to the Ato domain of expression, as well as in the R2/5 and R3/4 photoreceptors (Fig. 7A; Kimmel et al., 1990; Dokucu et al., 1996). Force-expression of ro under a heterologous heat-shock-responsive enhancer-promoter results in loss of neurogenesis, attributable to the repression of ato (Dokucu et al. 1996). However, more recent and exhaustive studies have since indicated that Ro more likely targets sens, aiding in the resolution of Sens from IGs to R8s, and later, repressing sens within R2/5 cells, preventing multiple cells from each growing ommatidium from acquiring R8 fate (Frankfort et al., 2001; Pepple et al., 2008).

Furthermore, ro expression requires DER signaling and recent analyses have revealed that Aop binds just upstream of the start site of ro transcription (Dominguez et al., 1998; Webber et al., 2013). This binding is substantiated by the recent publication of a transcriptome perturbation assay that predicts Aop regulation of ro (Potier et al., 2014). We observe that aop mutant clones exhibit widespread upregulation of Ro labeling, including the early and enhanced expression within the MF, as compared to WT tissue (Fig. 7B, arrows).

\section{ro rescues the reduced eye of the EgfrE1/aop interaction.}

The dominant DER allele Ellipse, $D E R$ Elp, elicits roughened eye patterning and weakened Ato expression that initiates normally but fails to form IGs. Similarly with aop mutant tissue, R8s occasionally and sporadically emerge from the diminished domain of Ato expression. Thus, enhanced or precocious DER activity opposes IG formation (Lesokhin et al., 1999). Although the effects of $D E R E l p$ on eye development are greatly muted in heterozygotes, as compared to reports with homozygotes, this background features weak Sens expression versus that of WT (Fig. 7C,D) 
and adult eyes that feature rough patterning and a mild reduction in size (Fig. 7E). In addition to overall reduced levels of Sens labeling in $D E R^{E l p}$ heterozygotes, Sens fails to express in a cluster of cells within IGs as in WT, in line with lost Ato labeling within IGs (Fig. 7C,D). This IG sensitivity is reflected in the reduced expression of the 5'-ato enhancer reporter in the $D E R^{E I p} /+$ background. Although strong expression is observed in R8s throughout the eyefield, the reporter is first induced not in clusters as it is in WT, but only within isolated cells (Fig. 7E').

Previous reports indicate that $D E R^{E l p}$ interacts with loss of function alleles of aop. Our findings agree that transheterozygous $D E R^{E l p}$ and $a o p^{1}$ interact to produce a severely reduced eye as compared to $D E R^{E l p} /+$ alone or $a p^{1} /+$ (Fig. 7E,F, $a p^{1} /+$ eye not shown). We reason that this genotype could permit ommatidial loss from either deficient R8 specification or loss of R8s at a later time, as has been observed in the spl allele of Notch (Li et al., 2003). The former would manifest as a loss of $5^{\prime}$-ato report within the MF, whereas the latter would have a fairly consistent induction of $5^{\prime}$-ato within the MF though it could be marked with a subsequent loss of report. Consistent with failed R8 formation, we observe only sporadic induction of the 5 -ato reporter near the MF in $D E R^{E l p} / a o p^{1}$ transheterozygotes (Fig. $7 F^{\prime}$ ).

To test the possibility that the $D E R^{E l p} / a o p^{1}$ phenotype might result from the combined misregulation of $r o$, we assessed the ability of the ro loss of function allele rox63 to rescue $D E R^{E l p} / a o p^{1}$ interaction. The introduction of $r o^{x 63}$ into the $D E R^{E l p} / a o p^{1}$ background elicits a stepwise rescue, wherein ommatidial count significantly increases from a $\mathrm{ro}^{+}$background to $\mathrm{ro}^{\mathrm{x} 63}$ heterozygotes and again to ro $^{x 63}$ homozygotes (Fig. 7F,G). Importantly, to test whether this phenotypic rescue was elicited at the time of R8 selection or at a later developmental checkpoint, we again used the $5^{\prime}$-ato enhancer reporter. The loss of report that was observed in DERElp $/ a o p^{1}$ eyes was rescued in the absence of Ro, as 5'-ato was restored across the MF and throughout cells of the posterior compartment (Fig. 7E'). From a macroscopic assessment of qualitative ommatidial patterning and size of eye field, DERElp heterozygotes are not rescued by loss of Ro (Fig. 7C,E, data 
not shown). However, this result is dubious and perhaps misleading as both $D E R^{E l p}$ and rox63 elicit similar adult eye defects, thus dampening our ability to assess any such rescue in the presence of WT aop.

\section{ro loss of function fails to rescue proneural mispatterning of aop mutant tissue.}

As a result of the precocious expression of ro in aop mutant tissue, we next assessed whether concurrent removal of both Aop and Ro could rescue the Ato and Sens defects reported in mutant clones of aop. If the upregulation of ro is alone responsible for the rapid degradation of Ato and Sens patterning, loss of Ro should rescue aop mutant eye tissue. Contrary to our expectations, dual clones of aop and ro fail to reliably rescue either Ato or Sens expression in the developing eye (Fig. 7H), suggesting that although Aop represses ro within the MF, this interaction is of limited consequence as it relates to proneural gene expression. However, from these data we cannot preclude the possibility that Ro and Aop may repress yet other negative regulators of Ato or Sens. roe may not be regulated by Aop.

roe is first expressed in the Drosophila eye, within and posterior to the MF, and later, is transiently expressed in R1/6 photoreceptors (St. Pierre et al., 2002; del Alamo and Mlodzik, 2008). As demonstrated with $E(s p l) m \delta$, Roe binds the enhancer of $\mathrm{Su}(\mathrm{H})$-regulated genes and blocks $\mathrm{Su}(\mathrm{H})$-mediated transactivation, although it is not clear the number of genes or degree to which Roe suppresses expression (del Alamo and Mlodzik, 2008). Though not fully established, it is likely that Roe is responsible for the repressive effects that create the dynamic staging that is characteristic of $\mathrm{Su}(\mathrm{H})$-responsive genes, as observed with immunolabeling against Aop and E(spl) (Fig. 3; Chapter 2).

To complicate matters, antibodies that were previously used in the detection of Roe are not publicly available, leaving only use of questionably effective gene traps of the MiMIC system (Venken et al., 2011). The MiMIC system makes use of a transposable element that randomly incorporates eGFP under control of a minimal enhancer-promoter. Ideally, fluorescence is achieved 
in the endogenous pattern of a gene into which the MiMIC construct has inserted. However, such an ideal is not always maintained due to the complexities associated with sophisticated enhancer regulation.

In the case of roe, a MiMIC insertion within the first intron $\left(r^{M I 07760}\right)$ elicits eGFP production starting within the MF, as shown against Arm and Sens staining (Fig. 8A). Although antibodies have demonstrated higher-level expression in the R1/6 cells, eGFP fluorescence persists uniformly throughout the posterior of the eye (del Alamo and Mlodzik, 2008; Fig. 8A). In aop mutant tissue, $r n^{\text {MiMIC }}$ report is unaffected (Fig. 8B). Given that this reporter fails to recapitulate WT expression of Roe, this data serves only as a modest guide to enhance our understanding of the aop-roe relationship. It is not clear how well this reporter responds to gene expression cues, specifically those that later restrict Roe expression. Regardless, Aop does not regulate the expression of $r n^{\text {MiMIC. }}$

\section{Barlacz is not regulated by Aop.}

Bar is expressed throughout undifferentiated cells of the posterior compartment of the developing eye, as well as within R1/6 retinulae (Higashijima et al., 1992; Lim and Choi, 2003). The Bar expression domain abuts that of Ato; all Bar-positive nuclei lack Ato and force-expression of Bar within the antennal disc results in the loss of Ato from corresponding cells (Lim and Choi, 2003). Furthermore, a recent transcriptome perturbation assay revealed that Aop may regulate the two repressors of the Bar locus, Bar-H1 and Bar-H2 (Potier et al., 2014). BarlacZ is used as an enhancer reporter in which a minimal promoter and lacZ is inserted between the two Bar transcription units, and reports in the developing retina similarly to the labeling of anti-Bar antibodies (Fig. 8C; Sato et al., 1999; Lim and Choi, 2004). We reason that if Aop prevents Bar expression during early Ato dynamics, we should expect precocious Bar activity in aop mutants. However, report from BarlacZ is absent from aop mutant tissue (Fig. 8D). These data are tempered by the fact that the lacZ report of Barlacz may not perfectly recapitulate the expression pattern of Bar-H1, the antigen to anti-Bar antibodies that were used to compare to the lacZ reporter. The anti- 
Bar-H1 antibodies recognize cells slightly anterior to the position of the BarlacZ reporter, suggesting the possibility of a slight difference in regulation between the reporter and Bar-H1 and that this difference may lead to inaccurate reporting in aop mutants. It remains a possibility that Aop might primarily target Bar-H2, a gene for which antibodies are not currently available.

\section{Bar is not upregulated in clones dually mutant for aop and ro.}

Lastly, it was necessary to test whether coincidental removal of both Aop and Ro elicits an upregulation of Bar, which might then repress Ato. This experiment was necessary given that Ro is predicted to regulate the genes of the Bar locus. Thus, it is plausible that cells mutant for both aop and ro might elicit ectopic Bar expression. To test this, we again monitored Bar expression through the use of the Barlacz fly line. Clones lacking both Aop and Ro failed to upregulate the Barlacz reporter in a manner that was similar to the loss of report in cells individually mutant for aop (Fig. 8E). Thus, our observations fail to provide any evidence for either direct or indirect regulation of the Bar locus by Aop.

\section{aop mutant clones do not affect timing of $E(s p l)$ expression.}

We next wanted to assess through clonal analysis whether Aop might regulate $E(s p l)$. Having demonstrated that Aop promotes IG formation through ato-3' while E(spl) simultaneously represses 5'-ato, we wished to observe whether Aop might affect the timing or levels of $E$ (spl) expression within the MF. Immunolabeling against $\mathrm{E}(\mathrm{spl})$ using $\mathrm{mAb323}$, which recognizes a subset of E(spl) bHLH repressors, reveals that Aop is not likely to repress $E(s p l)$ within the MF, as the timing of $\mathrm{E}(\mathrm{spl})$ expression in aop mutant tissue does not appear to deviate from that of WT, nor do the expression levels appear to vary (Fig. 9A,B, arrows). However, mutant analysis does not allow us to rule out the possibility that $E(s p l)$ could be only very minorly perturbed in aop mutants. $E(s p l)$ expression is dynamically regulated within the MF and the induction of aop clones appears to perturb the fine-scale patterning of the leading edge of $E(s p l)$ expression. Whereas WT E(spl) immunostaining often reveals a pattern of periodic projections of E(spl) that separate Ato IGs, such 
patterning appears to breakdown within aop clones (Fig. 9B). Thus, it is possible that in the absence of Aop, E(spl) might become misexpressed in cells that normally support robust enhancement of Ato to facilitate IG maturation.

\section{Force-expression of Aop does not result in E(spl) repression.}

To more directly test the possibility that Aop represses $E(s p l)$, we sought to force-express aop act, a derivative of Aop wherein MAPK target Ser residues have been mutated to Ala to prevent inhibition of Aop activity by MAPK. In wandering third instar larvae, the dppGAL4 driver produces GAL4 in developing antennae along both the dorsal and ventral margins and in a swath of cells that stretch between the two margins, across the center of the antennal disc. The UAS-GFP reporter indicates that with this driver, GAL4 is produced in a cluster of cells along the dorsal margin that significantly overlap with expression of $E$ (spl) (Fig. 9C). Aop is not expressed during this stage of antennal development. We reasoned that if Aop binds to and represses the expression of $E$ (spl), we would be likely to see reduced expression of $E(s p l)$ in the presence of exogenous Aop. The overexpression of Aopact resulted in a dramatic increase in the amount of E(spl) expression, as detected by mAb323 (Fig. 9D). The expression domain of E(spl) expanded toward the interior of the antennal structure, along with that of Ato and Sens (Fig. 9D, arrows; WT Ato not shown). Although these results do not directly negate the possibility of a relationship between Aop and $E(s p l)$, nor of a relationship between Aop and any single of $E(s p l)$ gene, it is instructive that Aop does not elicit an observable negative impact on $E$ (spl).

\section{Aop sensitizes IGs to repression by E(spl)}

Aop is required to maintain transcription from ato-3' during IG maturation. Aop operates in this role presumably from the induction of Notch signaling within the MF, where both Aop and $\mathrm{E}(\mathrm{spl})$ are similarly expressed. At this time, E(spl) represses 5'-ato, indicating that the continuous induction of ato-3' is primarily, if not entirely, responsible for Ato up to the latest stage of IG maturation where Roe directs repression of E(spl). Thus, failure to maintain Ato during IG 
formation also requires that $\mathrm{E}(\mathrm{spl})$ be present to prevent autoregulation. Therefore, the removal of both Aop and E(spl) should likely rescue Ato expression. Though observed at low levels, Ato is briefly expressed in aop mutants. Ato, in the absence of E(spl), precociously commits to autoregulation (Chapter 2). To assess this possibility, we labeled for Ato and Sens in tissue that was mutant for both aop and $E(s p l)$. As expected, both Ato and Sens were restored in double-mutant clones (Fig. 10A). Notably, expression was restored not in all cells, but was sporadic throughout mutant tissues (Fig. 10A, inset). This is perhaps an indication that autoregulation requires a specific minimal level of Ato expression and that this threshold is not attained in all cells within aop mutants.

Having demonstrated that aop loss of function sensitizes ato to repression by E(spl), we next assessed whether an aop mutant might enhance E(spl) gain of function. The hairyH10 enhancer trap (hH10 GAL4) drives GAL4 expression anterior to and within the MF. With this driver, forceexpression of $\mathrm{E}(\mathrm{spl}) \mathrm{m} \delta$ elicits a reduced eye and, frequently, an anterior cleft that is reminiscent of the furrow-stop phenotype and, molecularly, features aberrant Ato labeling (Fig. 10B; Chapter 2; Ligoxygakis et al., 1998). Consistent with a role in facilitating premature Ato repression, mutant aop significantly enhances the eye defect that results from force-expressed E(spl)m $\delta$ (Fig. 10C).

\section{DISCUSSION}

\section{Aop is integrated into Ato patterning and IG maturation.}

The Ato patterning process is a sophisticated coupling of inductive and repressive genetic signals, starting with the induction of the 3' enhancer, which elicits the expression of Ato and subsequent autoregulation via the 5' enhancer. Logically, the restriction of Ato must initiate with the $3^{\prime}$ enhancer and later be followed by the limitation of $5^{\prime}$-ato expression. Previous reports have indicated that Aop is required for proper expression of Sens and normal patterning of the retina. In this study we have provided further evidence that aop mutants disrupt eye patterning one step prior to Sens induction, during the maturation of Ato-positive IGs. Previous studies rule out the 
possibility that the loss of Sens within aop mutants is responsible for the observed loss of Ato. Although clones of mutant sens fail to maintain stage-4 Ato, such mutant tissues exhibit otherwise WT IG maturation (Frankfort et al. 2001).

Previous studies have suggested that Aop maintains WT retinogenesis through a direct interaction with Arm. Accordingly, the subsequent accumulation of Arm opposes proper neurogenesis through the induction of Wg pathway signaling components. However, Arm upregulation and enhanced apical constriction are also common to neurogenic phenotypes, as shown in the case of both $S u(H)$ and $E(s p l)$ mutants, with the former also lacking Aop. Thus, we sought to explain the aop phenotype through an Arm-independent mechanism.

\section{Aop represents a node of crosstalk between Notch and DER, as indicated by Ro.}

The possibility of a role for DER in the resolution of R8s has been subject to debate.

Although we fail to present evidence of a direct link between Aop and DER-MAPK activity, we were able to detect indirect interaction between Notch and DER via the presence of ectopic of Ro within aop mutant tissues. Our findings agree with previous efforts that have indicated that Ro requires DER activity for its expression in the eye (Dominguez et al. 1998). Thus, Aop is regulated by DER in the developing eye, and removal of Aop results in wide-scale misregulation of Ro, a result that suggests two points. First, Aop opposes DER within the MF, though it is not clear if this occurs in WT. Second, Notch signaling, through the induction of aop, imposes a delay on at least this one DER-dependent gene. This is suggestive of further interplay between Notch and DER wherein Notch utilizes Aop to delay the effects of DER-Ras-MAPK signaling within the MF. Therefore, Notchdependent processes, such as IG formation, can fully mature prior to the activation of a restrictive signal.

Although clonal analysis failed to identify other gene(s) directly responsible for the aop phenotype, the $D E R^{E I p} / a_{o p}{ }^{1}$ interaction still implicates Ro as a DER-driven effector of R8 fate. Rather than informing our understanding of the aop clonal phenotype, the ro mutant rescue of 
$\operatorname{DerElp}^{\mathrm{a} a p^{1}}$ indicates that $D E R^{E l p}$ is sensitized to early Ro expression, perhaps due to ancillary effects of precocious DER pathway activity that is further exacerbated through interaction with aop. Unexplored in this work are the possibile effects that Aop loss might have on the FMR2/AF4 ortholog lilliputian (lilli). Lilli permits high-level expression of Ato's cognate dimerization partner Daughterless (Da) to facilitate ato autoregulation (Distefano et al., 2012). Through a mechanism that remains unknown, Lilli also promotes proper induction of ato-3' (Distefano et al., 2012). Furthermore, lilli mutants enhance the eye defects of $D E R^{E l p}$ flies, suggesting the possibility that aberrant MAPK activation within the MF can create an environment that sensitizes ato-3' to premature inactivation (Tang et al., 2001).

\section{MAPK activity may oppose Aop.}

We have shown that Aop is strongly coexpressed with Ato only within maturing IGs, during a phase of Ato expression that we characterize as early stage-2. Furthermore, Aop is absent from cells of late stage-2 IGs until after R8 resolution is achieved (Fig 3; Fig. 7). The onset of stage-3 signifies the start of ato repression. Coincident with the onset of stage-3, MAPK becomes rapidly activated (Chen and Chien, 1998). As stage-3 progresses, the number of cells that label for Ato from each original IG progressively decrease as the same cells from which Ato is lost become labeled for $\mathrm{E}(\mathrm{spl})$ (Chapter 2). Although $\mathrm{E}(\mathrm{spl})$ is present in these cells, Aop is not, which is likely a reflection of the presence of pMAPK and its degradative effects on Aop (Fig. 7; Rebay and Rubin, 1995). Though less probably, the loss of Aop from these cells might also be due to E(spl) activity. Previous studies indicate that force-expression of E(spl)M7 and M8 result in downregulation of aop enhancer reporter activity, suggesting that $\mathrm{E}(\mathrm{spl})$ may repress both ato and aop in during stage-3, in a mechanism that is perhaps enhanced by pMAPK (Rohrbaugh et al., 2002; Ramos et al., 2003).

Roe likely represents a primary mode of transcriptional regulation of aop in the MF. Roe is an inhibitory component of Notch signaling that selectively binds to $\mathrm{Su}(\mathrm{H})$-activated enhancers and subsequently attenuates transcription (St. Pierre et al., 2002; del Alamo and Mlodzik, 2008). Roe is 
expressed within the MF and to its posterior; removal of Roe results in failed 5'-ato (Melicharek et al., 2008). As such, data currently suggest that Roe is active within late-IGs to suppress $E$ (spl) expression, thereby promoting that of Ato (del Alamo and Mlodzik, 2008; Chapter 3). Presumably, this mode of regulation likely extends to that of other $\mathrm{Su}(\mathrm{H})$-responsive genes such as aop. Accordingly, the potential attenuation of aop would not result in a loss of Ato as it coincides with the downregulation of E(spl). Furthermore, the timing of the loss of Aop from mature IGs strongly supports Roe regulation of aop. Although Aop is removed from IGs during late stage-2, pMAPK is not observed until later, when Ato begins to transition from stage-2 to stage-3. Thus, Roe likely accounts for the temporal discrepancy between Aop loss and the activation of MAPK.

The sudden removal of Aop from late-IGs fails to address why ro is not expressed in late-IGs. The lack of Ro in such cells suggests the possibility that various levels of DER signaling might initiate a staggered series of genetice consequences. In such scenario, low-level DER signal strength might first downregulate aop, followed by a second-tier signaling event in which Ro and other repressors become activated. Previously, tiered DER activity has been proposed to govern events during R8 resolution wherein DER may, at low levels, delay repression of Ato and at increasingly higher levels alter Notch signaling activity (Lesokhin et al., 1999). It is also possible that Ro is subject to repression within IGs by a secondary factor, such as Roe, a possibility that also suggests that $\mathrm{Su}(\mathrm{H})$ might contribute to transcriptional regulation of Ro. Regardless of involvement by Roe, the absence of Aop alone is not sufficient to permit ro expression.

\section{A mechanism for the regulation of ato}

Our data support a model in which Aop permits maintenance of ato-3' in early and mid-IGs, even as $\mathrm{E}(\mathrm{spl})$ concomitantly represses $5^{\prime}$-ato, as validated by the premature activation of $5^{\prime}$-ato in the absence of E(spl) (Fig. 11A, Chapter 2). Thus, the early E(spl) activity demonstrates that Aop coexpression with Ato is made possible through continued induction of ato-3'. This is suggestive of a mechanism in which Aop, through targeting a yet unidentified repressor, permits ato-3' 
expression until Roe is able to downregulate E(spl). This further implies that if DER is to have a role in the regulation of ato and R8 resolution, it would be through its regulation of Aop and, indirectly, the ato-3' enhancer. This model is consistent with the excessive activity of ato-3' in the absence of Ato or Da (Chapter 4; Melicharek et al., 2008). In either mutant background, ato is expressed at high levels but the 5' enhancer does not initiate. In addition to a lack of Notch activity in either ato or $d a$ mutant backgrounds, MAPK does not become activated. Additionally, we have previously shown that canonical Notch signaling does not significantly impact report from ato-3', further suggesting that Ato-Da activity may induce DER-MAPK with minimal support from the Notch pathway.

We propose a mechanism of Ato expression that consists of four distinct regulatory phases (Fig. 11B). In phase I, only ato-3' is targeted for induction, via products of the RDGN. Phase II presents mixed regulation the ato enhancers, wherein both the $3^{\prime}$ and 5' enhancers are poised for activation, yet only the 3' enhancer elicits the production of Ato. At this time, the 5' enhancer is repressed by E(spl). Aop is required here to ensure continued ato-3' output, otherwise Ato would be extinguished as both enhancers succumb to repressive effects. In phase III, 5'-ato activity becomes established as Roe temporarily represses E(spl) (del Alamo and Mlodzik 2008). It is at this time that ato-3' may be diminished to yield strictly to ato autoregulation. The posterior margin of the ato-3' expression domain displays a degree of patterning into early IGs. Lastly, phase IV is marked by the activation of MAPK, which is accompanied by the restoration of $\mathrm{E}(\mathrm{spl})$ and the progressive resolution of Ato to a single R8 from each original IG. The total involvement of MAPK in resolving Ato remains unclear. In aop mutant backgrounds, the concurrent loss of Ro is insufficient to provide rescue; whereas the R8 defect of $D E R^{E l p} / a o p^{1}$ flies was fully rescued by mutant ro. Thus, additional forces are at play in the two genetic backgrounds in question. It remains to be tested whether the presence of Aop during IG maturation is required to buffer against 
low-level MAPK activity that may not be readily detectable via immunohistochemistrty or if Aop's role is to repress genes that are activated by other pathways, independent of DER-MAPK.

ETV6/TEL may mediate Notch-Ras signaling crosstalk in lymphopoiesis.

Lymphopoiesis relies upon the proper binary fate assignment of the common lymphoid progenitor (CLP), which assumes either B-cell or T-cell fate. CLPs are by default, genetically programmed to assume B-cell fate, which also requires increased reliance on Ras-MAPK signaling as compared to T-cells, especially in the early stages of fate assignment (Inlay et al., 2009; Irving et al., 2014; Iritani et al., 1997). T-cell specification is facilitated by CLP migration to the thymus, where Notch signaling directs CLPs to assume T-cell fate (Radtke et al., 1999; Pui et al., 1999). The absence of either NOTCH1 or $\mathrm{Su}(\mathrm{H})$ ortholog CSL/RBP-J is associated with development of B-cell acute lymphoblastic leukemia (B-ALL). Likewise, constitutive activation of NOTCH1 results in the formation of T-cell ALL (T-ALL; Radtke et al., 1999). Although Ras is used in T-cell development, constitutive activation can elicit T-cell defects that also result in T-ALL (Kindler et al., 2008). The Aop ortholog ETV6/TEL is frequently disrupted in cases of childhood ALL, with most ETV6 disruptions resulting in loss of function and the development of B-ALL (Raimondi et al., 1997). Similar phenotypic outcome from NOTCH1 and ETV6 mutation suggests the possibility of conserved regulation of ETV6 and Aop via Notch signaling. The role of aberrant Ras-MAPK signaling in B-ALL and T-ALL further implicates functional conservation of ETV6 and Aop in Notchmediated opposition to low level MAPK activity. Thus, Notch signaling and Aop/ETV6 may well represent a common node of Notch-Ras crosstalk that is applicable to a wide variety of developmental and disease cases.

\section{MATERIALS AND METHODS}

\section{Drosophila genetics}

Flies were cultured on yeast-glucose media at $24^{\circ} \mathrm{C}$ and maintained according to a typical diurnal schedule. aop $^{1}$ is resultant of a nonsense mutation G952A that disables DNA binding 
(Caviglia and Luschnig, 2013). $S u(H)^{\wedge} 47$ results from an imprecise P-element excision that removes transcription start sites of $\mathrm{Su}(\mathrm{H})$ and $\mathrm{l}(2) \mathrm{Bg} 35$ (Morel and Schweisguth, 2000). The rescue construct $P\{l(2) B g 35+\}$ obviates contributions of $l(2) B g 35$ to $S u(H)$ loss-of-function phenotypes described herein (Morel and Schweisguth, 2000). Similarly, $D f(3 R) E(s p l)^{b 32.2}$ removes the entire $E$ (spl) locus, including gro (Schrons et al., 1992). gro is rescued by the inclusion of $P$ \{gro+ $\}$ (Heitzler et al., 1996). aopyan1 is a moderate loss-of-function allele that results from imprecise P-element excision from aopyanP that removes cytological map points 22D5-E1 (Lai and Rubin, 1992). pnt ${ }^{\wedge} 88$ results from the imprecise P-element excision from $p n t^{1277}$, eliciting loss of $p h t-P 1$ transcription unit and 3' exon of pnt-P2 (Scholz et al., 1993). ato ${ }^{1}$ encodes A25T, K253N and N261I, the last of which putatively disrupts DNA binding (Jarman et al., 1994). $N^{55 e 11}$ results from a 3.5kb insertion within the 5' coding region of $\mathrm{N}$, eliciting a premature nonsense mutation (Kidd et al., 1986). DERElp encodes A877T, which confers enhanced sensitivity to receptor activation (Lesokhin et al., 1999). $r 0^{x 63}$ harbors a deletion in the $5^{\prime}$ coding region that results in frameshift after 30 aa (Kimmel et al., 1990). Reporter stocks used are as follows: $P\left\{w^{+m C}\right.$ ato5'F:9.3 $\}$ and $P\left\{w^{+m C}\right.$ ato3'F:5.8 $\}$ (Sun et al., 1998); Mi\{MIC\}rn ${ }^{M I 07760}$ (Venken et al., 2011); BarP058-lacZ (Sato et al., 1999). First instar larvae from which mosaic clones generated with $h s F L P$ were heat-shocked for $60 \mathrm{~min}$ at $37^{\circ} \mathrm{C}$ and tissues were harvested during late second/early third instar.

\section{Immunohistochemistry}

All steps were performed at room temperature unless otherwise indicated. Tissues were dissected in $0.1 \mathrm{M}$ sodium phosphate buffer and fixed in 4-6\% formaldehyde, $0.1 \mathrm{M}$ sodium phosphate buffer. Tissues were washed in $0.3 \%$ Trition, $0.1 \mathrm{M}$ sodium phosphate buffer bathed in primary antibody mixtures (antibody concentrations shown below in 1\% BSA, 0.1M sodium phosphate buffer) for $12-18$ hours at $4^{\circ} \mathrm{C}$. Primary antibodies were removed, tissues were washed in $1 \%$ BSA in $0.1 \mathrm{M}$ sodium phosphate buffer and bathed in secondary antibody mixtures $11: 1000$ dilution for each secondary, in 1\% BSA, $0.1 \mathrm{M}$ sodium phosphate buffer) for $2 \mathrm{hrs}$. Secondary 
antibody mixtures were removed, tissues were washed $0.1 \mathrm{M}$ sodium phosphate buffer. Tissues were mounted in 60\% glycerol and imaged using an Olympus Fluoview FV1000 Confocal microscope. All scanning data reported was observed in a minimum of tissues from 5 independent animals of like genotype.

Primary antibodies include mouse $\alpha$-Arm (1:100, Riggleman et al., 1990); rabbit $\alpha$-Ato (1:5000, Jarman et al., 1994); guinea pig $\alpha$-Sens (1:500-800, Nolo et al., 2000); mouse $\alpha$-Aop (1:200, Rebay and Rubin, 1995); mouse $\alpha$ - $\beta$-gal-40-1a (1:800-1000, Ghattas et al., 1991); rat $\alpha$-Ciact-2A1 (1:100, Motzny and Holmgren, 1995); mouse $\alpha$-Ro (1:100, Kimmel et al., 1990); mouse $\alpha$-E(spl)mAb323 (1:3, Jennings et al., 1994). Mouse $\alpha$-Arm, mouse $\alpha$ - $\beta$-gal-40-1a, rat $\alpha$-Ciact-2A1 were obtained from the Developmental Studies Hybridoma Bank, created by the NICHD of the NIH and maintained at The University of Iowa, Department of Biology, Iowa City, IA 52242. Tissues to be labeled with primary rabbit $\alpha$-Ato were dissected in $0.3 \%$ Triton, $0.1 \mathrm{M}$ sodium phosphate buffer.

Secondary antibodies used include 488-goat $\alpha$-mouse (Jackson), 488-rabbit $\alpha$-GFP (Life Technologies), 546-goat $\alpha$-rabbit (Life Technologies), 546-goat $\alpha$-mouse (Life Technologies), 546goat $\alpha$-Rat (Life Technologies), 633-goat $\alpha$-guinea pig (Life Technologies).

\section{Light Micrography}

Adult flies were mounted and promptly imaged using a Nikon digital camera mounted on a Leica MZ16 stereomicroscope. For facet counts totaling $\geq 700$, the value of 700 was used in all related calculations and reporting.

\section{Image Production}

All images were processed in Adobe Photoshop CC v. 14.2. Image manipulations of brightness/contrast and color balance were applied uniformly across each image shown. Images were then organized in Adobe Illustrator CC v. 17.1 before being exported to Microsoft Word v. 14.4.4. 


\section{ACKNOWLEDGEMENTS}

We are grateful to Justin Kumar, Nick Baker, Graeme Mardon, Yuh Nung Jan, Hugo Bellen, Sarah Bray, Andrew Dacks and Daniel Marenda, whose generous gifts of flies and antibodies have enabled these studies. 

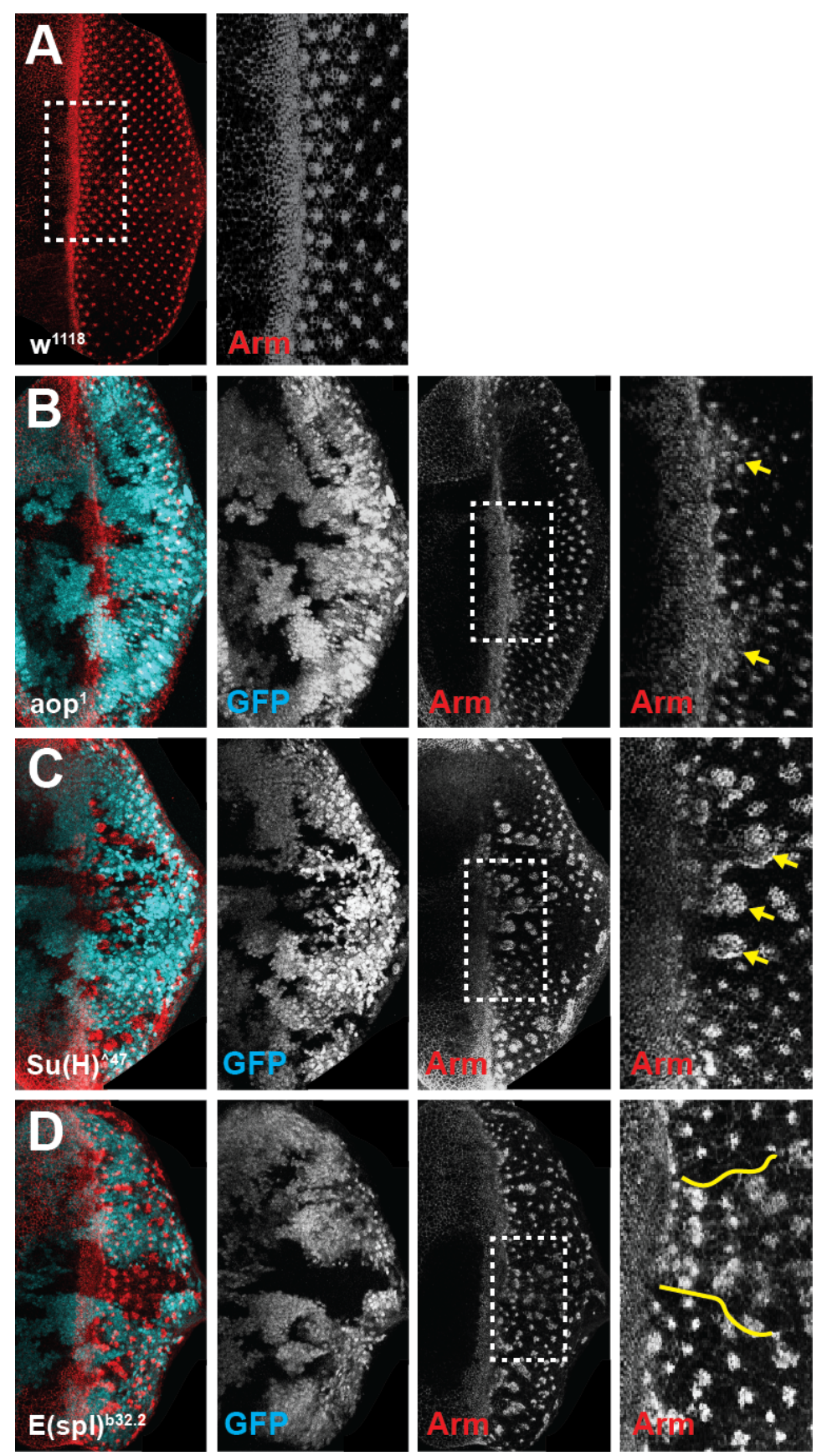

Fig. 1. Apical constriction is enhanced neurogenic mutants. (A) Arm (red) accumulates at adherens junctions, which are most highly concentrated within the MF and rosettes. Anterior is left. (B) aop mutant clones accumulate Arm. As for all clonal analyses, far left panel is merge, followed to the right by individual channels. Mutant tissues are marked by the absence of GFP. Inset at far right shows continued apical constriction of aop mutants (arrows). (C,D) Su(H) and $\mathrm{E}(\mathrm{spl})$ mutants, respsectively, display a similar Arm enhancement phenotypes. Arrows in C indicate several clusters of persistent apical constriction. Boundaries in D flank a large E(spl) clone. Note the persistent high-level background staining in $\mathrm{E}(\mathrm{spl})$ clones. Genotypes: (A) $w^{1118}$, (B) aop ${ }^{1}$ frt40A/ubiGFPnls frt40A; eyFLP/+, (C) Su(H)^47 frt40A P\{l(2)Bg35+\}/ubiGFPnls frt40A; eyFLP/+; (D) frt82B Df(3R)E(spl)b32.2 P $\left\{\right.$ gro $\left.^{+}\right\} /$frt82B ubiGFP eyFLP. 

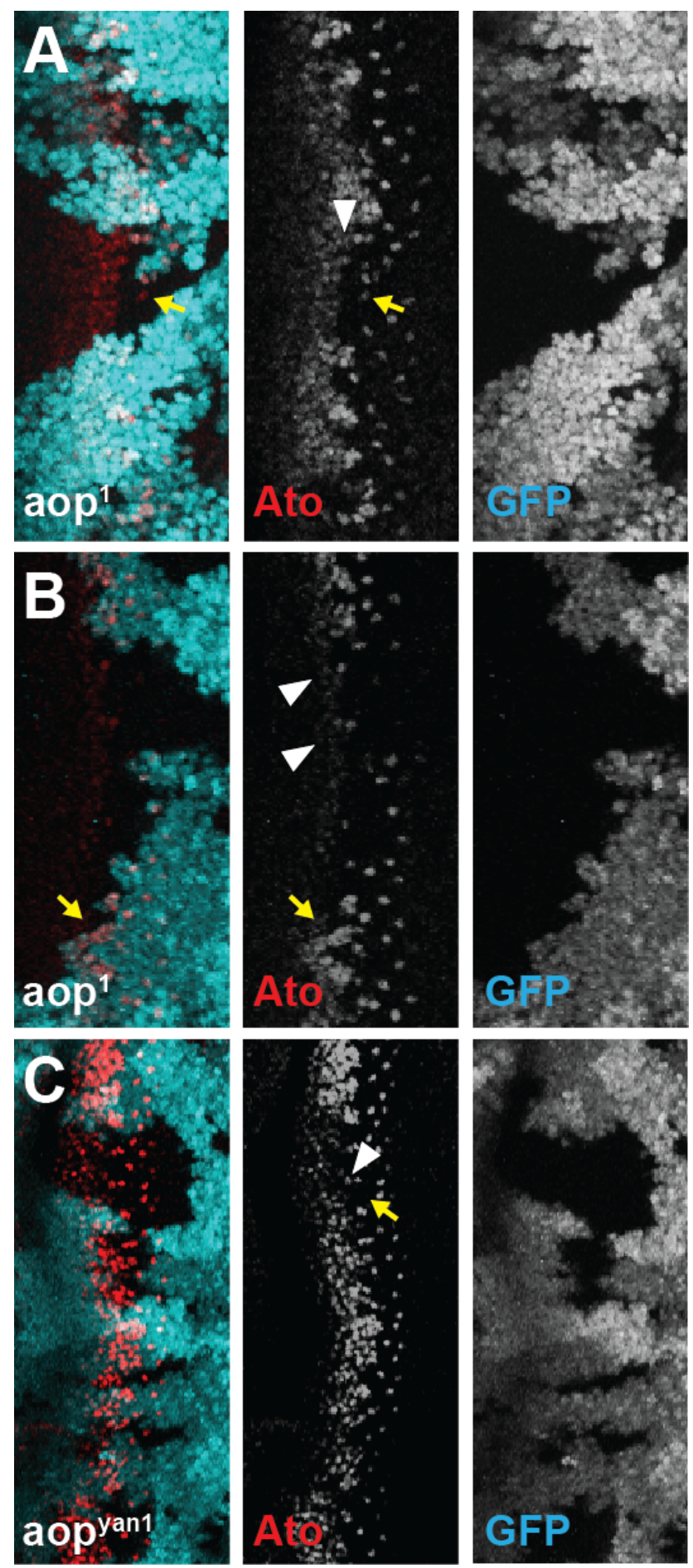

Fig. 2. Aop is cell-autonomously required for Ato IGs. (A-C) aop ${ }^{1}$ mutant clones stained $\alpha$-Ato. (A) Ato initiates normally in aop mutants but IGs fail to form as in WT (arrowhead). Despite loss of Ato, R8-like individuals are still occasionally observed (arrow). (B) R8-like cells emerge from the MF where IGs form in WT tissue (arrowheads). The reduction in Ato is cell-autonomous, as shown by a bisected IG that stains strongly for Ato only in WT cells (arrow). (C) The weaker mutant $a^{\text {ayan } 1}$ displays Ato defects that are less severe than in $a p^{1}$. IGs fail to form as in WT (arrowhead) and some R8s are absent (arrow). Genotypes: (A,B) aop ${ }^{1}$ frt40A/ubiGFPnls frt40A;eyFLP/+, (C) aopyan 1 frt40A/ubiGFPnls frt40A;eyFLP/+. 

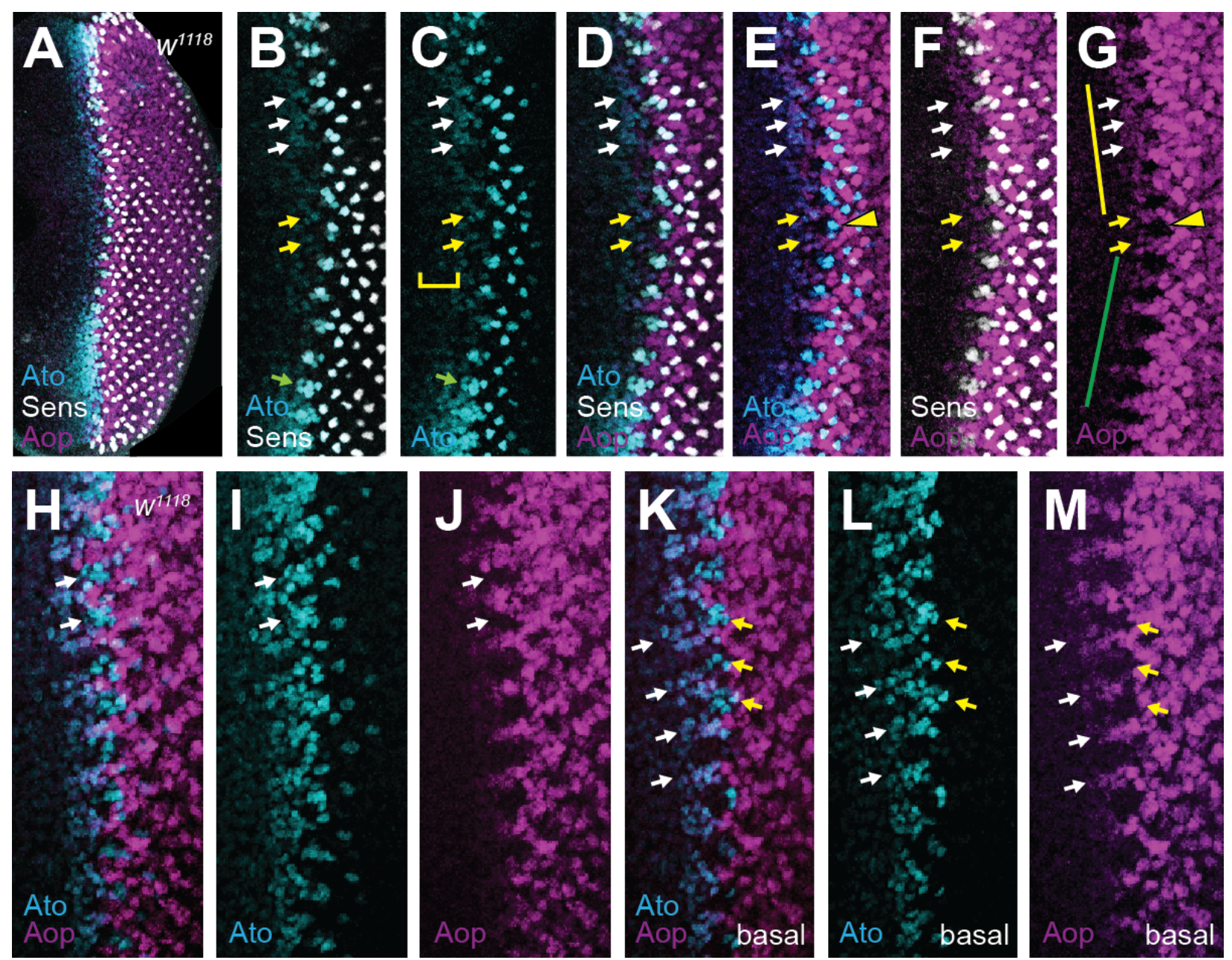

Fig. 3. Aop and Ato coexpress during early IG formation. (A-G) WT disc stained $\alpha$-Ato (cyan), $\alpha$ Sens (grey), $\alpha$-Aop (magenta). (B-G) Magnification of MF in A. Ato is patterned through four stages: 1 (bracket), 2 (green arrow), 3 (arrowhead), 4 (individual cells posterior to previous stages). IGs progressively form to the anterior of stage-3 Ato clusters (yellow arrows, younger clusters; white arrows, more mature). Aop is coexpressed with Ato during these stages of early IG formation (yellow, white arrows). As IGs fully mature (green arrow), Aop becomes lost from $\alpha$-Ato-stained cells. (G) Aop expression is dynamic, as some regions show anterior-most $\alpha$-Aop as a dorsoventral column (yellow bar), and others only show expression intercalated between IGs (green bar). Aop first initiates during early stage-2 (B-G, yellow, white arrows). As IGs mature and reach late stage 2, Aop is lost (H-J, white arrows). (K-M) Basal-most sections of H-J similarly reveal coexpression of Aop and Ato in anterior-most IGs (white arrows). Aop is lost from IGs as they mature (yellow arrows). Genotypes: (A-M) $w^{1118}$. 

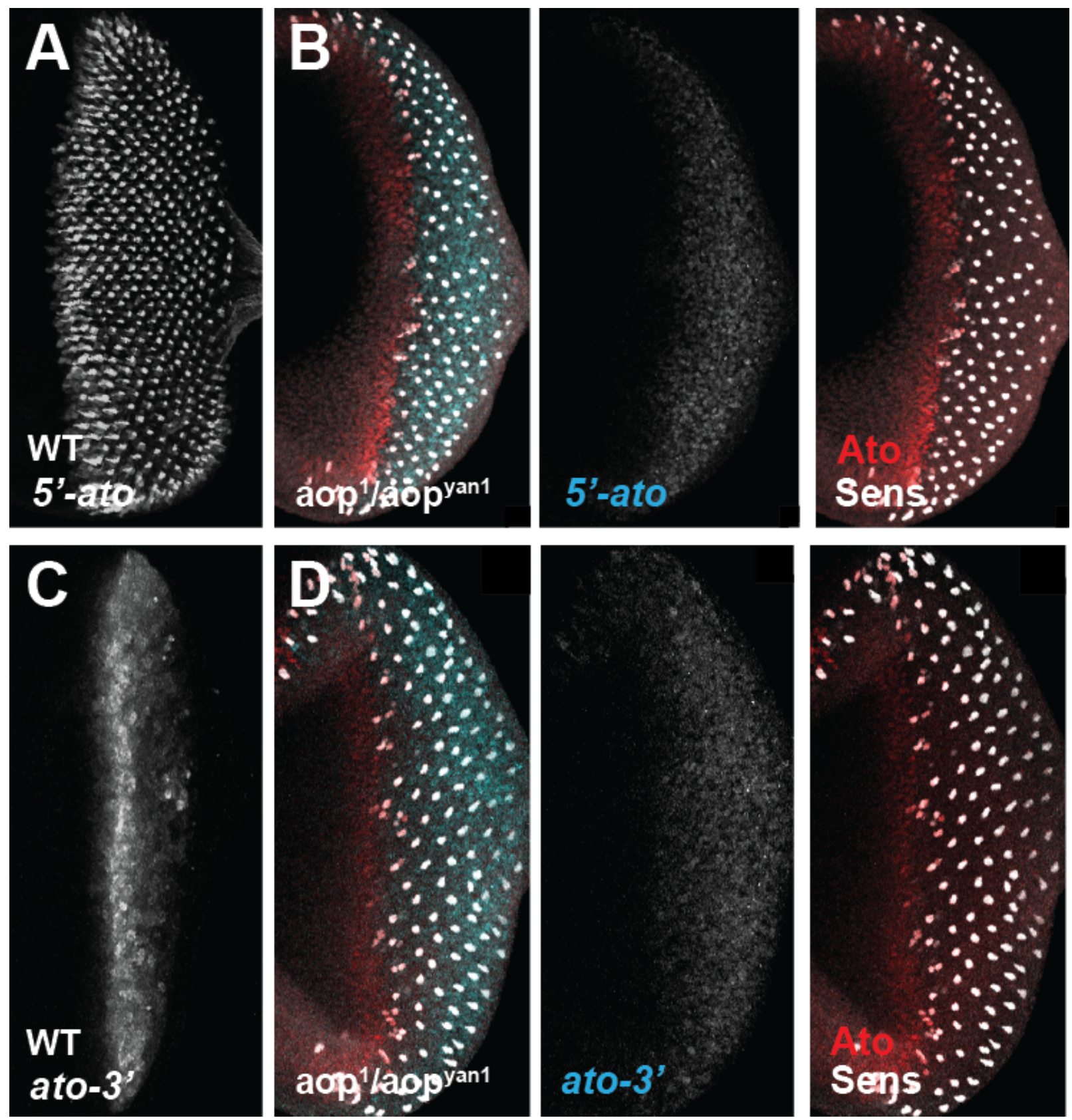

Fig. 4. aop loss-of-function perturbs both ato enhancers. (A) 5 '-ato-lacZ reports in IGs and R8s of WT eyes. (B) 5 -ato report in aop1/aopyan 1 transheterozygotes also stained $\alpha$-Ato and $\alpha$-Sens. 5 '-ato report is severely diminished, exhibiting only broad, basal level activity with no clear patterning in IGs or R8s. (C) ato-3'-lacZ report initiates just anterior of the MF, quickly peaks and decays throughout toward the posterior. (D) ato-3' report in aop1/aopyan1 transheterozygotes also stained $\alpha$-Ato and $\alpha$-Sens. Ato-3' report lacks the characteristic peak observed in the MF of WT. $(B, D)$ Ato and Sens IGs appear sporadically within MF and R8s are occasionally missing from posterior of eye. Genotypes: (A) $\mathrm{P}\left\{w^{+m C}\right.$ ato $\left.5^{\prime} F: 9.3\right\} /+$, (B) aop ${ }^{1}$ frt40A/aopyan1 $\mathrm{P}\left\{w^{+m C}\right.$ ato $\left.5^{\prime} F: 9.3\right\}$, (C) $\mathrm{P}\left\{w^{+m C}\right.$ ato3'F:5.8\}/+, (D) aop ${ }^{1}$ frt40A/aopyan1 $\mathrm{P}\left\{w^{+m C}\right.$ ato3'F:5.8\}. 

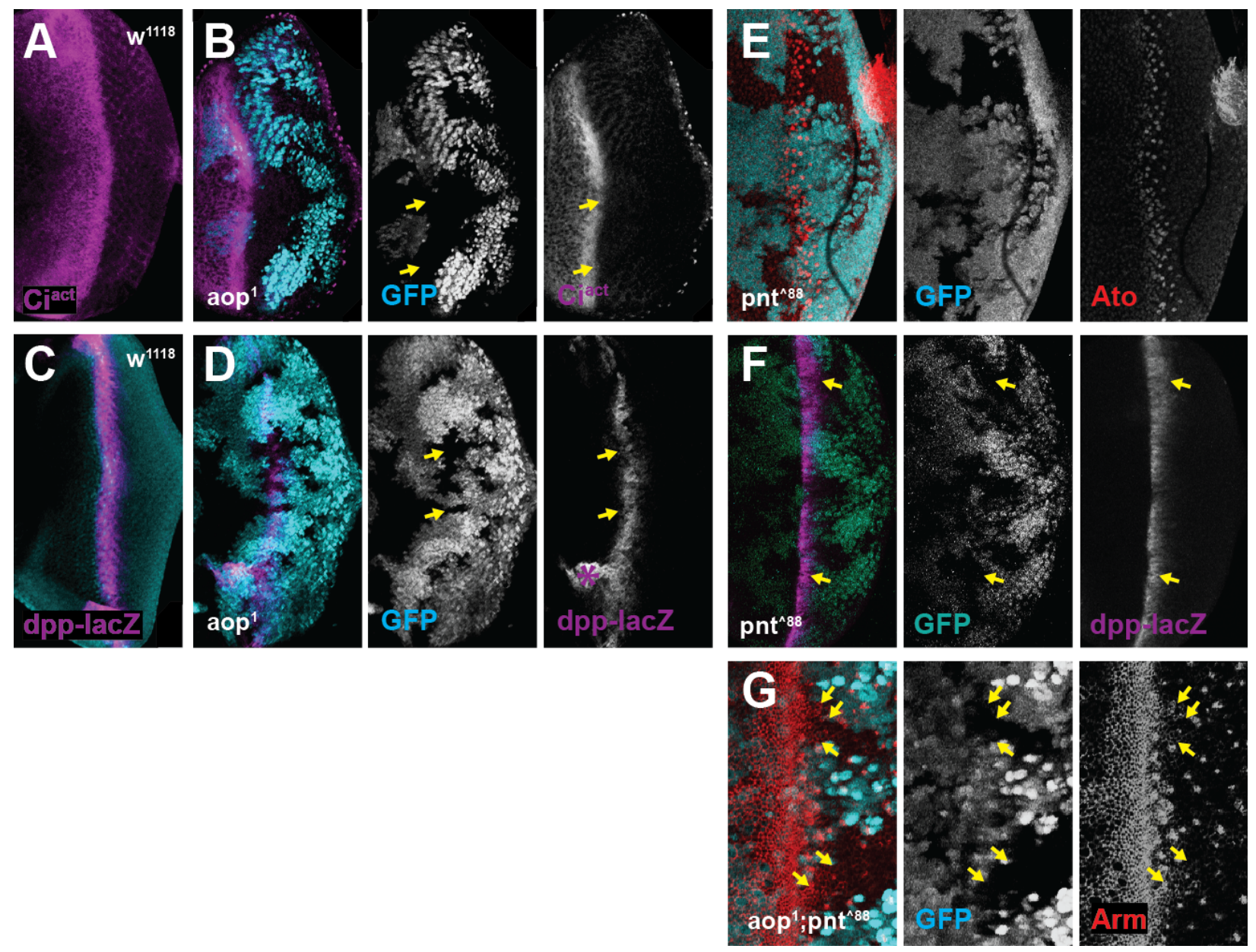

Fig. 5. aop mutation does not misregulate Ci processing and $\mathrm{dpp}$. (A) Ciact progressively accumulates anterior of the MF in WT discs in response to Hh signaling. (B) aop mutants do not affect $\mathrm{Ci}$ accumulation at the MF (arrows) or in the posterior of the eye. (C) dpp-lacZ reporter expresses in a relatively uniform dorsoventral stripe within the MF of WT discs. (D) dpp report is slightly downregulated but not abrogated in aop mutants. Asterisk $\left(^{*}\right)$ denotes a fold in the tissue. (E) $\alpha$-Ato staining reveals the emergence of R8s from the MF of pnt mutants. (F) dpp-lacZ report is unaffected in pnt clones (arrows). (G) $\alpha$-Arm enhancement and apical constriction are present in dual aop, pnt mutant cells, indicating that this phenotype is not the cause of enhanced Pnt activity in the absence of Aop. Genotypes: (A) w1118, (B) aop 1 frt40A/ubiGFPnls frt40A;eyFLP/+, (C) $\mathrm{P}\{$ dpp-lacZ $\} /+$ (chr. III), (D) aop ${ }^{1}$ frt40A/ubiGFPnls frt40A;eyFLP/P $\{$ dpp-lacZ $\}$, (E) frt82B $p^{\wedge} t^{\wedge} 88 /$ frt82B ubiGFP eyFLP, (F) $\mathrm{P}\{d p p$-lacZ $\} /+; f r t 82 B$ pnt ${ }^{\wedge} 88 /$ frt82B ubiGFP eyFLP, (G) aop 1 frt40A/ubiGFPnls frt40A;frt82B pnt ${ }^{\wedge 88} /$ frt82B ubiGFP eyFLP. 

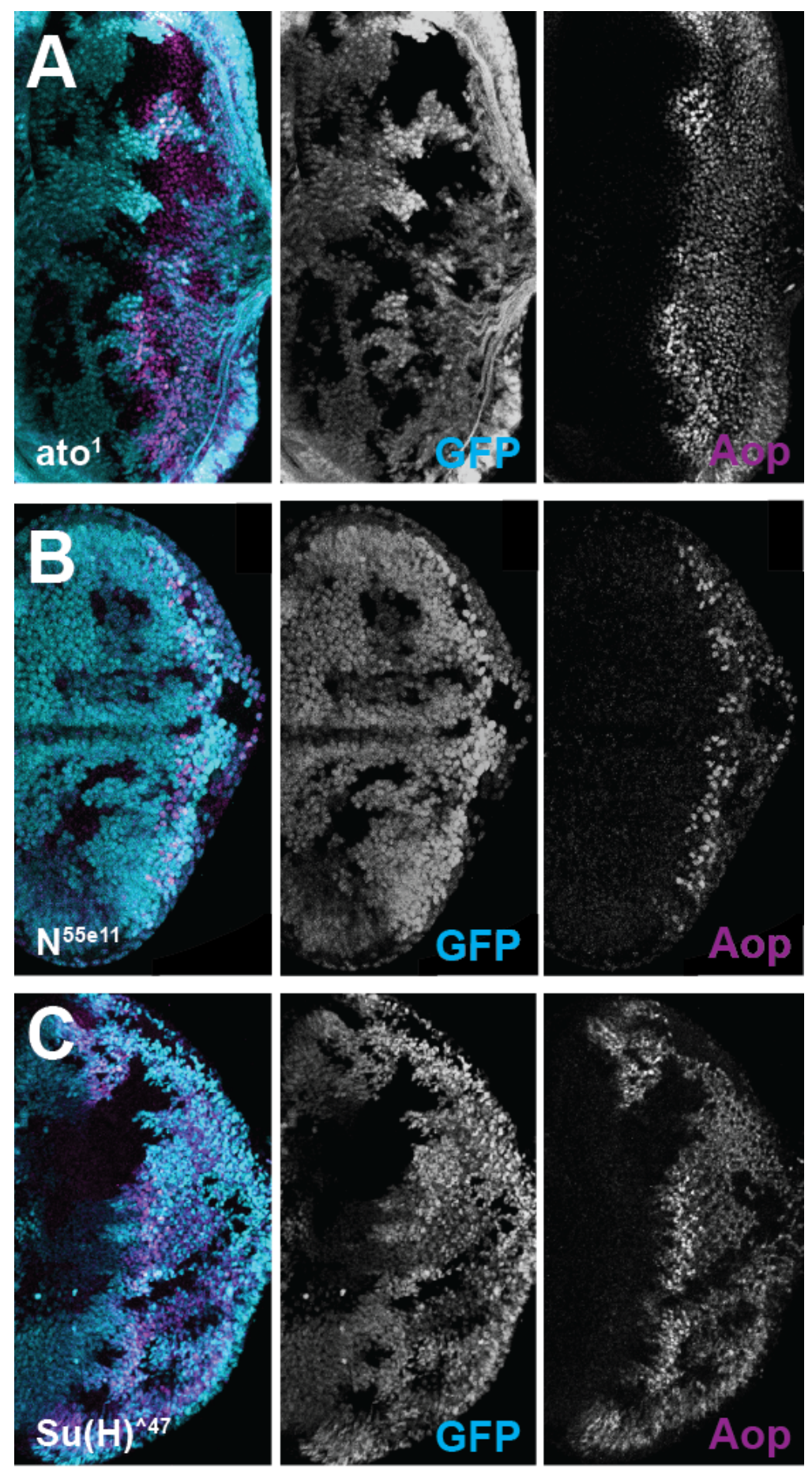

Fig. 6. Aop requires Notch signaling for its expression in eye discs. (A) ato mutant cells exhibit non-autonomous, delayed Aop expression. Overall, ato1 cells fail to properly induce Aop as early occurs in WT. (B) Removal of Notch elicits cell-autonomous loss of $\alpha$-Aop staining nuclei. (C) In the absence of $\mathrm{Su}(\mathrm{H}), \alpha$-Aop labeling is not detected. Near the MF, Aop follows direct autonomous control by $\mathrm{Su}(\mathrm{H})$, whereas the the posterior of the eye features non-autonomous loss of Aop from

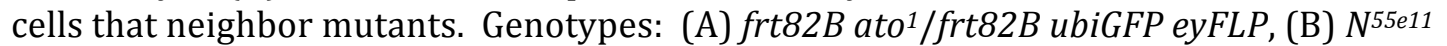
frt19A/ubiRFP hsFLP frt19A, (C) Su(H)^47 frt40A P $\{1(2) B g 35+\} /$ biGFPnls frt40A; eyFLP/+. 

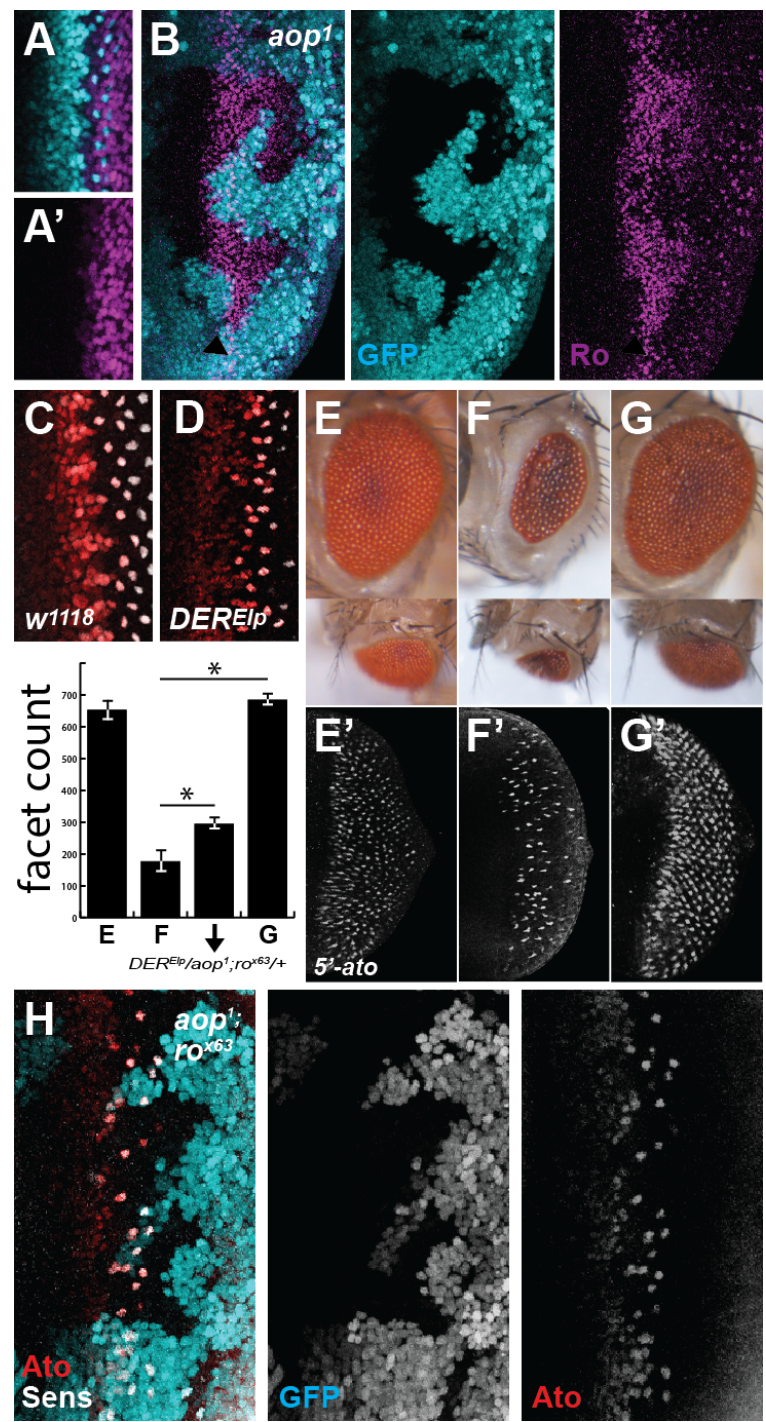

Fig. 7. Aop represses $r o$ in the MF. (A,A') $\alpha$-Ato and $\alpha$-Ro staining in WT eye discs. Ro complements the posterior margin of Ato. (B) In aop mutant clones, $\alpha$-Ro labels more broadly and further anterior than in WT cells. (C) In WT eyes, $\alpha$-Ato and $\alpha$-Sens strongly co-label IGs and early columns of R8s. (D) Normal IGs fail to form in DERElp heterozygotes. Regardless, R8s still regularly emerge from the MF. Note that DERElp homozygotes exhibit more severe Ato and R8 loss (Lesokhin et al., 1999). (E-G) Adult eye phenotype of (E) $D E R^{E l p} /+$, (F) $a o p^{1} / D E R E l p$, (G) $a o p^{1} / D E R^{E l p}$;ro ${ }^{x 63}$ flies. Eyes are reduced in $a o p^{1} / D E R^{E l p}$ flies, but are rescued by the removal of Ro. Quantitation of adult eye facets reveals stepwise rescue of $a o p^{1} / D E R^{E l p}$ through ro loss-of-function. (E'-G') 5-ato-lacZ report corresponding to genotypes in $\mathrm{E}-\mathrm{G} ; \mathrm{n} \geq 10$, asterisk $\left(^{*}\right)$ denotes $\mathrm{P}<0.001$. (E') Report in $D E R^{E l p}$ heterozygotes is similar to WT (Fig.4A) with loss of clustered expression at MF. (F') Report is missing through much of $a o p^{1} / D E R^{E l p}$ eye disc, including at the MF. (G') The removal of Ro rescues 5 'ato report in $a o p^{1} / D E R^{E l p}$ flies. (E-G) Quantitation of genotypes $a o p^{1} / D E R^{E l p}$ rescue. Asterisk $\left(^{*}\right.$ ) indicates $\mathrm{P} \leq 0.001, \mathrm{n} \geq 10$. (H) Dual clones of $a o p$ and ro mutants stained $\alpha$-Ato and $\alpha$-Sens. Ato phenotype is similar to that observed in individual aop mutants (Fig. 2). Genotypes: $(\mathrm{A}, \mathrm{C}) w^{1118}$, (B) aop ${ }^{1}$ frt40A/ubiGFPnls frt40A;eyFLP/+, (D, E) DERElp/+, (E') P $\left\{w^{+m c}\right.$ ato5'F:9.3\} DERElp/+, (F) aop $1 / D E R E l p,\left(\mathrm{~F}^{\prime}\right)$ aop 1 frt40A/P $\left\{w^{+m c}\right.$ ato5'F:9.3\} DER Elp (G) aop ${ }^{1}$ DERElp/+;frt82B ro ${ }^{x 63}$, (G') aop ${ }^{1}$ frt40A/P $\left\{w^{+m c}\right.$ ato $\left.5^{\prime} F: 9.3\right\}$ DER Elp; frt82B ro ${ }^{x 63},(\mathrm{H})$ aop ${ }^{1}$ frt40A/ubiGFPnls frt40A;frt82B ro ro $^{x 63} /$ frt82B $^{2}$ ubiGFP eyFLP. 

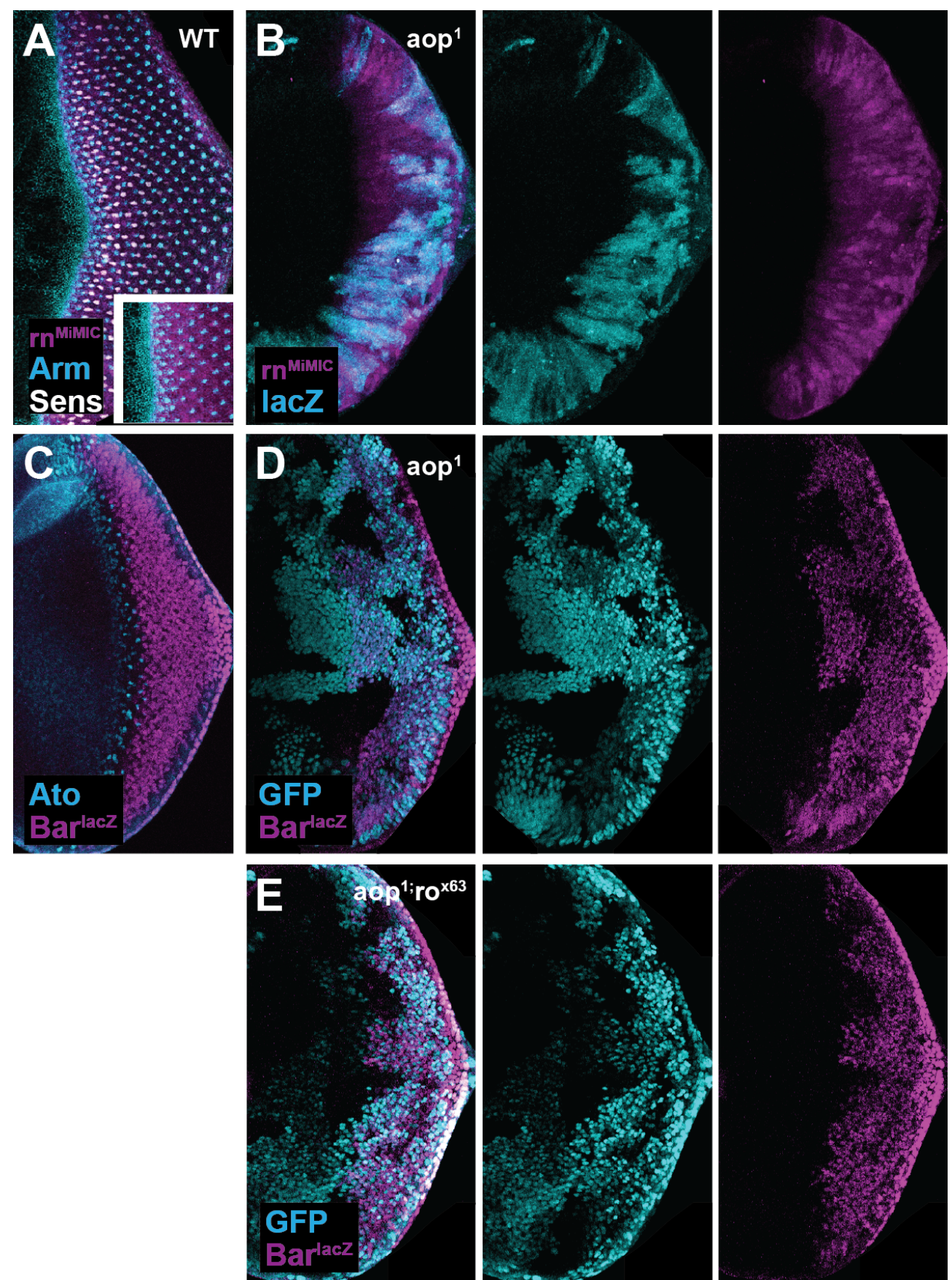

Fig. 8. Aop does not regulate roe or Bar reporters. (A) A MiMIC transposon insertion in the roe locus facilitates eGFP (magenta) expression in the MF and posterior eye disc. The MF is marked by enhanced $\alpha$-Arm (cyan) and $\alpha$-Sens (white). (B) aop mutants, marked by the absence of lac $Z$ expression do no perturb $r^{M i M I C}$ report. (C) BarlacZ (magenta) report initiates posterior to Ato (cyan), and persists throughout the posterior eye. (D) Aop is required for the report from Barlacz. Mutant cells are marked with GFP. (E) Dual aop, ro mutants do not rescue Barlacz expression. Mutant cells are marked with GFP. Genotypes: (A) Mi\{MIC $\} r^{M I 07760} /+$, (B) aop ${ }^{1}$ frt40A/ubiGFPnls

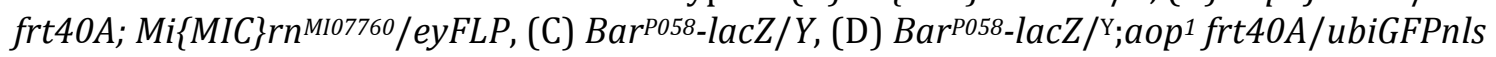
frt40A;eyFLP/+, (E) Bar P058-lacZ/Y;aop ${ }^{1}$ frt40A/ubiGFPnls frt40A;frt82B rox63/frt82B ubiGFP eyFLP. 

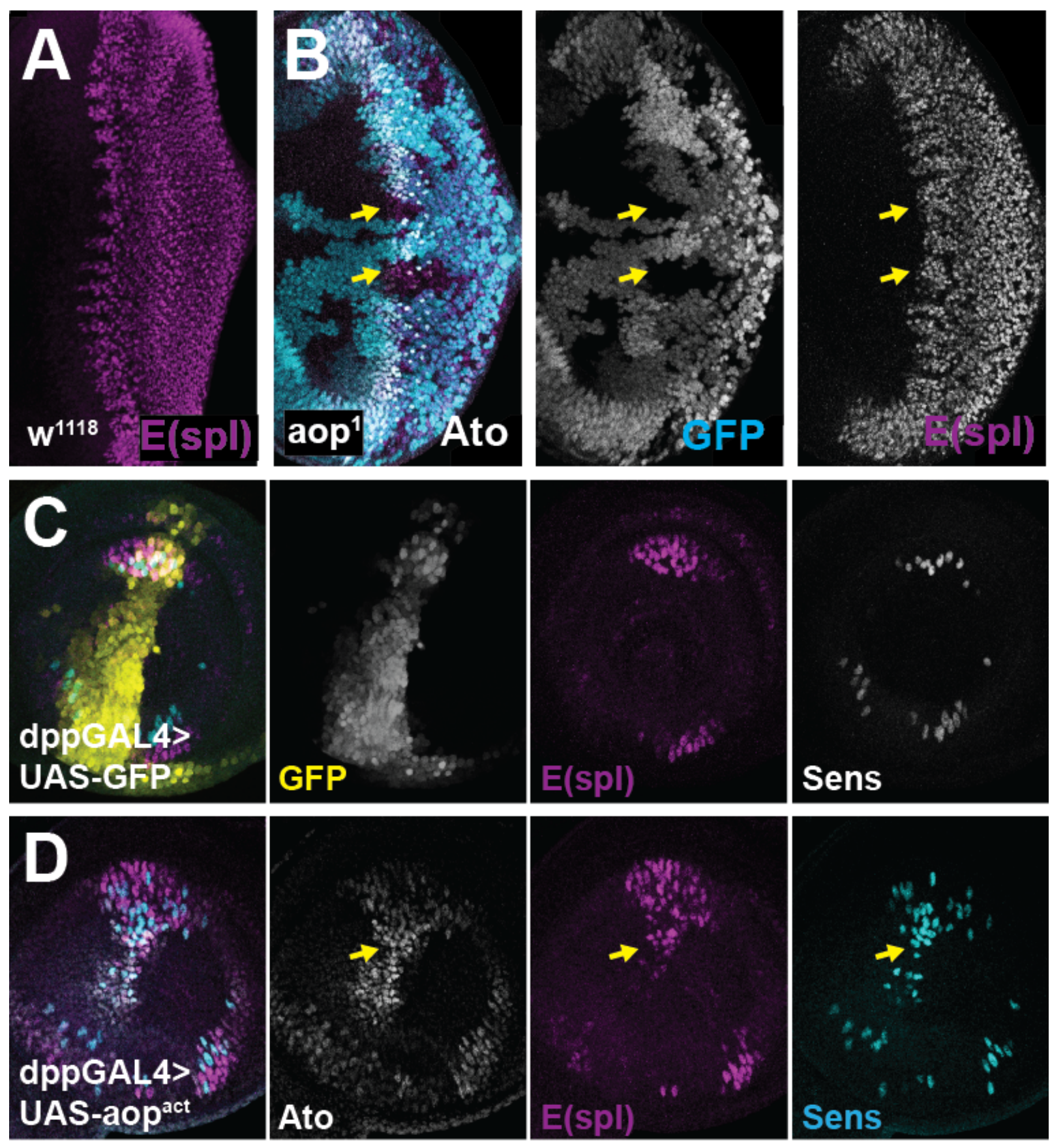

Fig. 9. Aop does not regulate $\boldsymbol{E}(\boldsymbol{s p l})$. (A) E(spl) (magenta) is expressed in the MF and throughout the posterior eye disc. (B) Mutant aop clones exhibit WT $\alpha$-E(spl) staining (arrows). $\alpha$-Ato marks the approximate location of the MF. (C) $d p p$ GAL4 drives UAS-GFP (gold) report at the dorsal, ventral margins and interior of antennal discs in wandering third instar larvae. E(spl) (magenta) overlaps with GFP at the dorsal margin. Sens (grey) expresses at the dorsal margin and two regions of the ventral margin. (D) $d p p$ GAL4 drives constitutively active Aop (UAS-aopact). Ato (white), $\mathrm{E}(\mathrm{spl})$ (magenta) and Sens (cyan) are expanded into the interior antennal disc Aopact is forceexpressed (arrow). Genotypes: (A) w1118, (B) ) aop ${ }^{1}$ frt40A/ubiGFPnls frt40A;eyFLP/+, (C) P $\{U A S$ GFP-nls $\} /+; P\left\{G A L 4-d p p\right.$-blk1\}/+, (D) P\{w ${ }^{+m c} U A S$-aop $\left.{ }^{a c t}\right\} / P\{U A S-G F P-n l s\} ; P\{G A L 4-d p p-b l k 1\} /+$. 

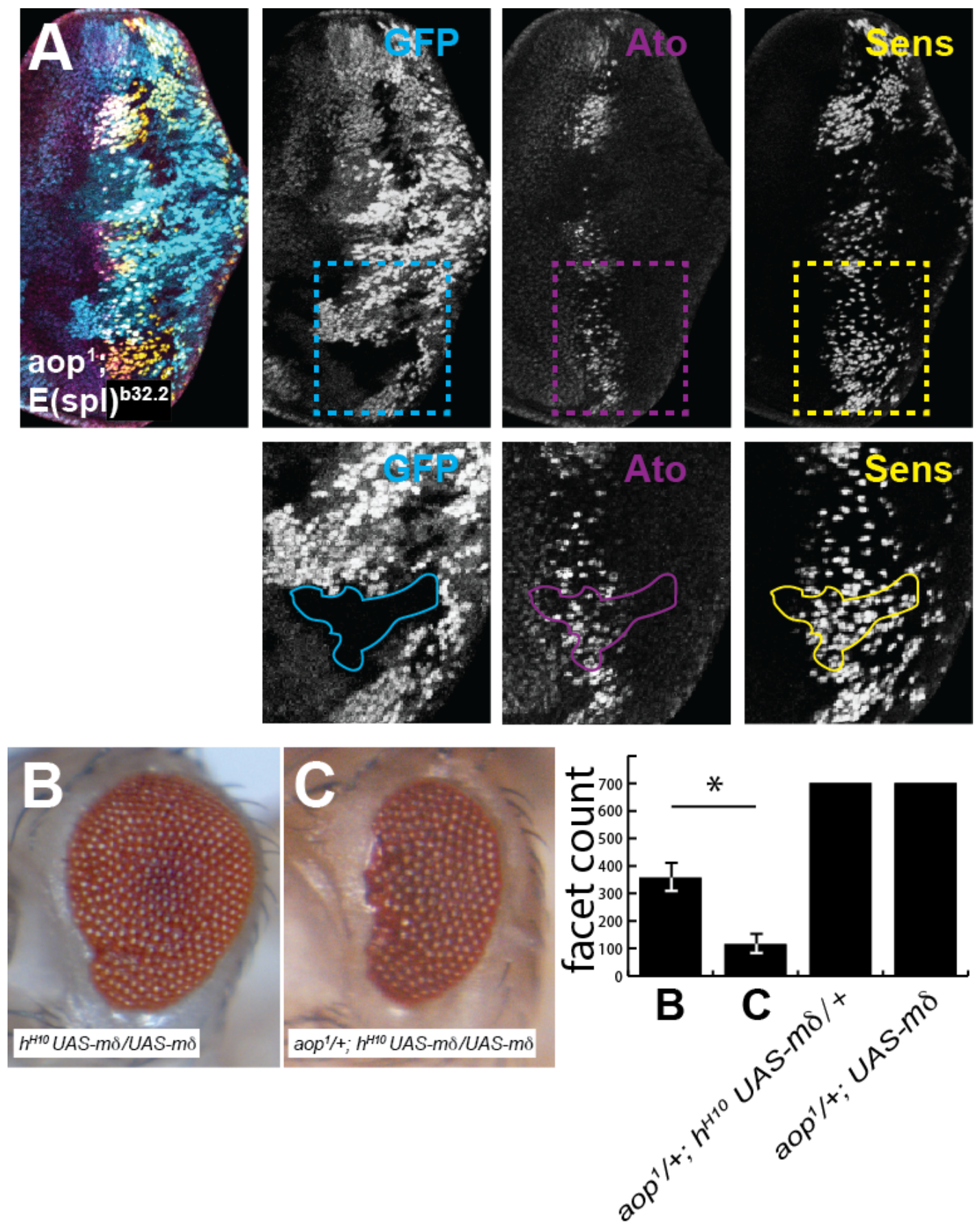

Fig. 10. aop loss-of-function sensitizes the eye to E(spl). (A) aop, E(spl) dual mutant clones feature $\alpha$-Ato (magenta) and $\alpha$-Sens (gold) staining, as opposed to reduced $\alpha$-Ato in aop clones (Fig. 2). Insets focus on large dual mutant clone, note sporadic Ato with various expression levels and broad expression of Sens, indicating that most aop, E(spl) mutants are specified as R8s. (B) $h^{H 10}$ $G A L 4$ driving two copies of transgenic $U A S-E(s p l) m \delta$. The eye is reduced, mispatterned and features a slight cleft at the anterior margin. (C) The introduction of aop loss-of-function to the genotype in $B$ further reduces the eye. Quantitation to the right of $C$; $n \geq 10$, asterisk $\left(^{*}\right)$ indicates $\mathrm{P}<0.001$. Genotypes: (A) aop ${ }^{1}$ frt40A/ubiGFPnls frt40A;frt82B Df(3R)E(spl) ${ }^{b 32.2} P\left\{\right.$ ry $^{+t 7.2}=$ gro $\} /$ frt82B ubiGFP eyFLP (B) $h^{H 10} G A L 4 P\left\{w^{+m c} U A S-E(s p l) m \delta\right\} / P\left\{w^{+m c} U A S-E(s p l) m \delta\right\}$, (C) aop ${ }^{1}$ frt40A/+; $h^{H 10} G A L 4$ $P\left\{w^{+m c} U A S-E(s p l) m \delta\right\} / P\left\{w^{+m c} U A S-E(s p l) m \delta\right\}$. 
A

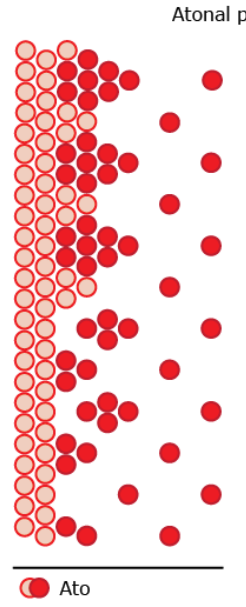

Ato, Aop Coexpression

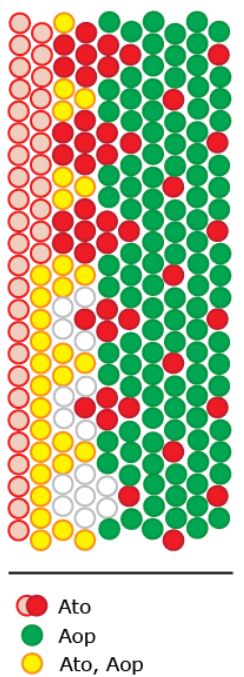

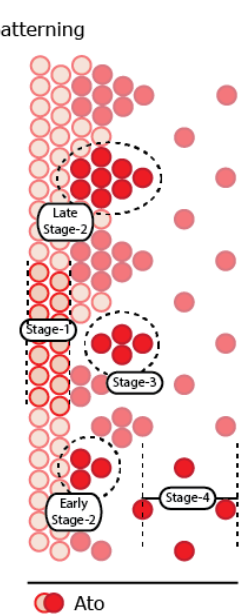

Aop only

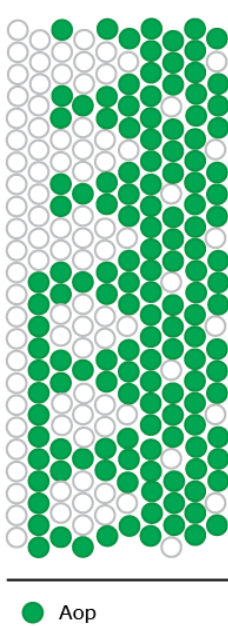

Aop

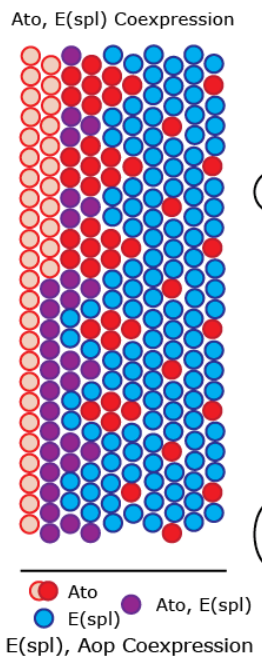

B
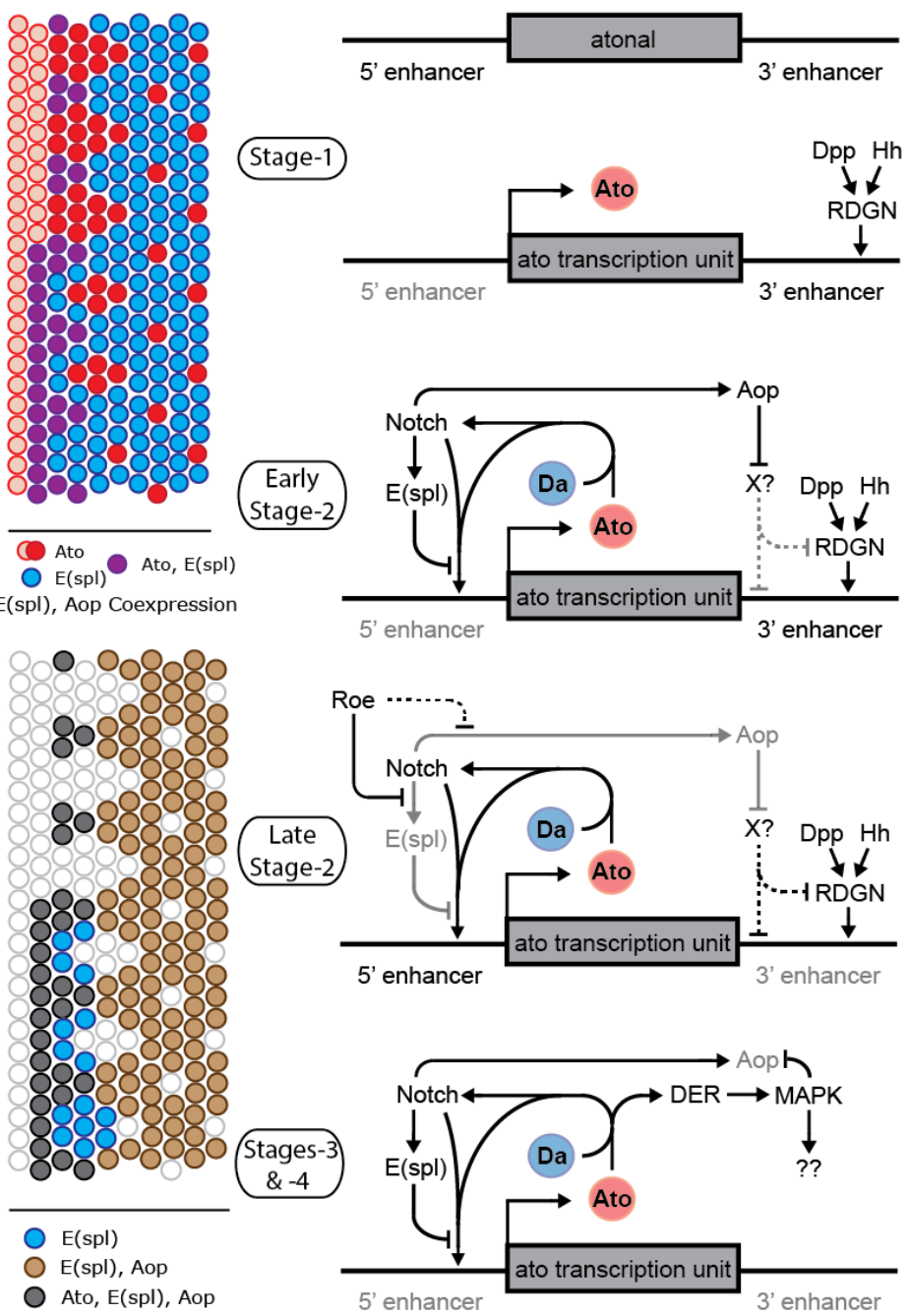

Fig. 11. Aop indirectly opposes E(spl) repression of ato during IG formation. (A) Ato coexpresses with E(spl) and Aop during early stage-2. Ato patterning commences with stage-1. Subsequently, phased clusters exhibit progressively increasing Ato expression throught early and late-stage2. Ato is subject to repression during stages-3 and -4 as it is resolved to R8s. E(spl) coexpresses with Aop during only early stage-2; expression of both is lost as IGs continue their maturation to late stage-2. Similarly, Aop and Ato colabel only early stage- 2 cells. The restoration of $\mathrm{E}(\mathrm{spl})$ accompanies the onset of stages- 3 and -4 , but Aop remains absent for longer from cells that were previously part of IGs. (B) ato is regulated by two enhancers, 5'-ato and ato-3'. Stage-1 expression is driven by RDGN transcription factors that are stimulated by morphogens. Stage-2 commences with Ato-Da-driven Notch signaling. As a result, both E(spl) and Aop are induced. $\mathrm{E}(\mathrm{spl})$ represses 5'-ato as Aop promotes continued expression from ato-3'. Though the mechanism for this maintenance remains unclear, we propose that Aop maintains ato-3' activity through either of two means: (1) repression of a direct repressor of ato-3' or, (2) a mechanism that remodulates RDGN activity on ato-3'. In late stage-2, Roe downregulates E(spl) and presumably, though not confirmed, Aop. As Ato autoregulation initiates, 5-'ato becomes active and ato-3' loses activity. Aop is absent from stages- 3 and -4 and $\mathrm{E}(\mathrm{spl})$ represses 5 'ato. MAPK is active in these cells, thus it is possible that MAPK may prevent accumulation of Aop in these cells. 


\section{Chapter 4: Ato facilitates anteroposterior transition through three distinct mechanisms.}

\section{ABSTRACT}

Drosophila retinogenesis is enabled by the anteroposterior compartmentalization of the eye anlage during second larval instar. Compartmentalization is enabled by the secretion of the Hedgehog $(\mathrm{Hh})$ from nascent neurons that form at the posterior margin of the eye disc. Hh acts anteriorly, facilitating anterior cells' transition into the posterior compartment. The full scope of the proneural Atonal (Ato) function in this process had been unexplored. In this study we demonstrate that Ato proactively promotes anteroposterior transition through its effect on three distinct signaling processes. Ato, in conjunction with Daughterless (Da) and Notch signaling, drive ato autoregulation. Independent of Notch, Ato and Da promote the downregulation of the retinal determination master regulator Eyeless (Ey), and stoppage of the ato-3' enhancer. Lastly, Ato regulates $d p p$-lacZ independently of Da or Notch, suggesting a novel function for class II bHLHs, a grouping to which Ato belongs. These findings indicate that Ato's role in eye development is not so passive as previously understood. Ato is active in the transformation of anterior compartment identity through its effect on dpp signaling, and alters the transition to posterior compartment identity through both Notch-dependent and Notch-independent processes. 


\section{INTRODUCTION}

Drosophila retinogenesis is the paradigm for studies of genes and signals in relation to pattern formation. During the second larval instar, the retina becomes anteroposteriorly compartmentalized. Neurogenesis is housed in the posterior compartment and the anterior allows cells to progressively attain neural competency. Retinal patterning occurs in the wake of the morphogenetic furrow (MF), a dorsoventral groove that travels anteriorly across the developing retina, separating the anterior compartment from the posterior. The MF initiates at the posterior margin of the eye anlage, where it is promoted by Hh signaling (Hh, Borod and Heberlein, 1998). Hh elicits two responses to facilitate neurogenesis: the induction of the proneural atonal (ato) and secondly, the expression of Decapentaplegic, Dpp, a secreted morphogen of the TGF- $\beta$ superfamily (Pappu et al., 2005; Strutt and Mlodzik, 1997). Dpp provides positional information to cells further anterior in the eye disc, preparing such cells to become competent to respond to Hh (Greenwood and Struhl, 1999). As neurogenesis proceeds, nascent photoreceptors secrete Hh, forming a cyclical feedback loop in which the two morphogens, Hh and Dpp, provide the impetus for anterior progression of the MF and subsequent differentiation (Rogers et al., 2005).

This cyclical feedback loop requires proper retinal patterning which is dependent upon regulated expression of Ato (Jarman et al, 1994; Sun et al, 1998). Ato is a class II bHLH that works in concert with its heterodimerization partner Daughterless (Da), a class I bHLH (Jarman et al., 1994; Brown et al., 1996). Ato exhibits a complex expression pattern that is sculpted by the effects of Hh, Dpp and Notch signaling (Zhang et al., 2006; Tanaka-Matakatsu and Du, 2008; TanakaMatakatsu et al., 2014; Sun et al., 1998). The effects of these signals are mediated through two separable ato enhancers located at opposite sides of the transcription unit; referred to as the ato-3' and 5'-ato enhancers (Sun et al., 1998). ato-3' is activated at the leading edge of the MF by genes of the retinal determination gene network (RDGN), whose activity require inputs from both $\mathrm{Hh}$ and Dpp (Pappu et al., 2005; Tanaka_Matakatsu and Du, 2008; Firth and Baker, 2009; Baker and Firth, 
2011). Once expressed, Ato stimulates Notch signaling and regulates its own expression via 5'-ato within clusters termed intermediate groups (IGs; Sun et al., 1998; Baker et al., 1996). IGs are then refined to single cells through Notch and $\mathrm{Su}(\mathrm{H})$-mediated induction of the Enhancer of split (E(spl)) bHLH repressors (Ligoxygakis et al., 1998; Lesokhin et al., 1999; Li and Baker, 2001). Each isolated Ato-positive cell becomes an R8, the first photoreceptor subtype to be specified within the developing retina (Jarman et al., 1994). All other ommatidial cell types are subsequently recruited to the R8 through inductive signaling (reviewed in Kumar 2012).

Once ato autoregulation is achieved, it is not clear if or how Ato might regulate changes to the RDGN. The anterior compartment of the eye attains neural competence through the dynamic expression of RDGN transcription factors (Bessa et al., 2002; Firth and Baker, 2009). Retinal identity is specified primarily through the action of the master regulatory (homeotic) gene Eyeless (Ey), which, in conjunction with Dpp signaling input, directs appropriate activation of other RDGN components. This culminates in the induction of Ato through direct binding of the tripartite transcription factor Eyes absent-Sine oculis-Dachshund (Eya, So, Dac; Tanaka-Matakatsu and Du, 2008; Pappu et al., 2005). Dac and So require Hh signaling for their expression anterior to the MF (Firth and Baker, 2009). Within the MF, Ey becomes downregulated whereas Dac continues to be expressed in undifferentiated, non-neuronal cells (Halder et al., 1998; Mardon et al., 1994). Continuous expression of Ey antagonizes neurogenesis, but only when expressed throughout the MF (Atkins et al., 2013). Thus, activities within the MF likely impose negative regulation on Ey to promote neurogenesis.

Dpp is expressed in a narrow dorsoventral band of cells within the MF (Blackman et al., 1991). Such expression is critical to the renewal of the pre-proneural region that is just anterior to the MF (Bessa et al., 2002; Firth and Baker, 2009). The pre-proneural region is where cells of the anterior compartment assume competency to respond to Hh (Greenwood and Struhl, 1999). Multiple reports and reviews indicate that Hh drives the expression of Dpp in a mechanism that is 
seemingly independent of other genes that are essential to neurogenesis (Kumar, 2012; Vivekanand and Rebay, 2006). Such reports indicate that Hh signaling, through its effector $\mathrm{Ci}$, is responsible for Dpp expression (Strutt and Mlodzik, 1996; Strutt and Mlodzik, 1997). However, Hh signal response also regulates the induction of Ato, which subsequently enacts a wide variety of changes (Greenwood and Struhl, 1999). A full dissection of the relationship between Hh, Ato, Notch and Dpp is thus required to more fully understand how morphogen cycling drives MF progression.

Ultimately, it remains unclear the degree to which Ato and Notch cooperate to enact the many changes that occur within and shortly after the arrival of the MF. Ato displays autoregulatory properties and a complex dependence on Notch pathway activity, but the full duties solely attributable to Ato and Da activity have yet to be more fully assessed. To probe this question, we have used FLP-FRT-mediated mitotic recombination to observe the various effects that $a t o$, $d a$ and Notch signaling mutants have on MF movement, reorganization of the RDGN, and Dpp expression. To answer those questions we first had to molecularly dissect the relationship between Ato and Notch and the effects that both exert on the two enhancers of ato. To little surprise, our analysis indicates that the concerted activity of Ato-Da and Notch signaling are required for ato autoregulation and to support MF progress. However, Ato-Da suppress ato-3' independently of the Notch pathway. Additionally, Ato-Da synergy is required to abrogate Ey expression in the MF, whereas Notch signaling is dispensable for this function. Lastly, Ato is required for Dpp expression independent of either Notch signaling or Da, indicating a novel function for a class II bHLH. Thus, Ato operates through three mechanisms, (1) in conjunction with $\mathrm{Da},(2)$ in its elicitation of Notch signaling, and (3) independent of Da to facilitate the various changes that are required for cells to transition from the anterior compartment to the posterior. The requirement of Ato for Dpp is unique among current bHLH observations in that Ato, a class II bHLH, appears to operate independently of its analogous class I heterodimerization partner Da. In addition to producing a revision to the model of MF progression wherein Ato bridges RDGN activity to both neurogenesis 
and Dpp expression, our data suggest a novel role for Ato and perhaps the opportunity to further understand inter- and intra-class interactions of bHLHs.

\section{RESULTS}

\section{Ato coexpresses with both $\mathrm{Ci}^{\mathrm{act}}$ and $\mathrm{dpp}$ report.}

To better appreciate the role of Ato with respect to the dynamics of other signaling effectors and MF-related genes, we first assessed the position of Ato expression as it relates to Eyeless (Ey), activated Cubitus interruptus (Ciact) and the $d p p$-lacZ reporter. Ey is a primary director of RDGN activity, exerting its influence on the expression of many other RDGN genes prior to the passage of the MF (Kumar, 2009). Given its early role in RDGN dynamics, Ey induction is independent of both

Hh and Dpp signaling (Firth and Baker, 2009). Far anterior to the MF, Ey comprises one component of a tripartite repressor that opposes retinal specification (Bessa et al., 2002). The onset of Dpp signaling in the anterior compartment hails changes to RDGN activity, freeing Ey from the repressor complex; culminating in the formation of the pre-proneural region just anterior to the MF, where cells achieve competence to respond to Hh (Greenwood and Struhl, 1999). The Hh response can be monitored in eye development by the accumulation of full-length $\mathrm{Ci}(\mathrm{Ciact})$, which is normally subject to cleavage into a trans-repressor form in the absence of Hh ligand (Pappu et al., 2003). Loss of Hh pathway components results in delays to MF progression, Ato expression, and loss of Dpp-reporter expression (Greenwood and Struhl, 1999; Strutt and Mlodzik, 1997).

As previously described, the anterior retinal compartment shows strong Ey expression that, with the arrival of the MF, quickly tapers to background (Fig. 1A; Atkins et al., 2013). Ato can be observed as a progression through several distinct phases of patterning, starting adjacent to the anterior of the MF and continued toward the posterior in clusters of high-level expression (Fig. 1A). Ato transitions from broad, dorsoventral (DV) expression into clusters as Ey expression drops to background levels (Fig. 1A, inset). Mirroring the expression of Ey, Ciact is observed anterior to and within the MF, and is not lost until the appearance of Ato clusters. As Ato progresses to a point 
where IGs can be discerned from the preceding DV stripe, $d p p$-reporter expression is first observed (Fig. 1B). Ciact and report from $d p p$-lacZ concur within a narrow margin of Ato positive cells (Fig. 1B, red bar of inset). Thus, in WT tissue, Ey epistatically precedes Ciact accumulation, which then precedes Ato and yet later $d p p$ activity. Ey is required for direct activation of Ato, but this analysis prompted us to speculate whether report from $d p p-l a c Z$ is dependent of Ciact, Ato, a downstream effector of Ato, or a combination of such factors.

\section{Ato exerts opposing effects on the 3' and 5' enhancers.}

Prior to assessing the role of Ato and Notch in the regulation of these MF-specific genes, we first had to define the relationship between ato, Notch signaling and the enhancers of ato. We next sought to assess the direct effect that Ato bears on each of its enhancer modules through use of the 3' and 5' enhancer-reporter constructs (Sun et al., 1998). ato ${ }^{1}$ mutant cells elicit broad upregulation of Ato (Fig. 2A; Chen and Chien, 1999; Lim et al., 2008) The ato ${ }^{1}$ mutant lacks DNAbinding via a point mutation in its basic domain, allowing the mutant protein to maintain antigenicity to antibodies developed in the lab of Yuh Nung Jan (Jarman et al., 1994). We next tested ato regulation at the level of each separate enhancer. 5'-ato directs expression in IGs and emergent R8s (Fig. 2F). Unsurprisingly, ato exhibits cell-autonomous autoregulation via the 5' enhancer (Fig. 2G), in agreement with previous studies of whole-retina mutants (Powell et al., 2004). In opposition to this effect, loss of Ato results in greater stimulation of 3' enhancer activity than in WT (Fig. 2D). In WT, ato-3' is expressed in the MF (Fig. 2C). $\beta$-gal perdurance elicits a report that fades through the posterior of the eye disc (Fig. 2C). Note that Fig. 2D features a high

contrast setting to dampen the intensity of ato-3' report; the enhancer is expressed in WT tissue as well, though this image does not clearly portray such.

\section{Da and Ato similarly regulate ato-3' and 5 '-ato.}

Previous analyses have described similar regulation of the ato enhancers in mutants of $d a$, the heterodimerization partner of Ato (Melicharek et al., 2008; Brown et al., 1996). Da is the only 
identified E-type bHLH activator of the Drosophila genome, strongly suggesting that any DNAbinding Ato activity is enacted in conjunction with Da. As such, the similarity of enhancer phenotypes in both ato and da mutants likely reflects that the two synergistically regulate activation of the 5' enhancer and repression of the 3' enhancer and that such an effect is the result of transcriptional regulation. Ato-Da dimers act as transactivators and presumably regulate the 5' enhancer through direct DNA-interaction. However, negative regulation of the 3' enhancer suggests an indirect mechanism; activated either directly by Ato-Da dimers or Notch signaling. Notch signaling regulates 5 '-ato.

Notch signaling is a complex regulator of ato. Though not required for 3' enhancer induction, Notch is required for the establishment of late Ato pattern effects, including the highlevel expression of Ato in intermediate groups and R8s that is characteristic of 5' enhancer activity (Sun et al., 1998; Baker and Yu, 1997; Baonza and Freeman, 2001). To test this assumption cellautonomously, we assessed enhancer activities in clones that are dually mutant for Drosophila Notch ligands, Dl and Ser. As expected, Notch pathway activity is required for activation of the 5' enhancer (Fig. 2H) but has only a mild repressive effect on 3' enhancer activity (Fig. 2E). Thus, Ato and Da act independently of Notch in the negative regulation of the 3' enhancer (Melicharek et al., 2008). Regardless, Notch signaling exhibits an absolute requirement in facilitating ato autoregulation but Notch only tunes regulation of ato-3'.

\section{$d p p$-reporter expression requires Ato, but not Da}

$d p p$-lacZ is a widely used reporter line for the assessment of cells of the developing eye that express and presumably secrete Dpp (Blackman et al., 1991). Previous analyses have shown that Dpp exhibits a requirement for Hh signaling via the receptor Smo (Strutt and Mlodzik, 1997). However, the expression of Ato and several other MF-associated genes also require Hh signaling for their proper timing. This introduces a previously overlooked dilemma as to whether Dpp is regulated solely by Hh signaling or by Hh in conjunction with factors expressed within the MF 
(Greenwood and Struhl, 1999). In the developing eye, Hh signaling relieves repression by Cirep, foregoing any requirement for Ciact-mediated transactivation (Pappu et al., 2003). In such event, Dpp would be immediately expressed upon the loss of Cirep. However, Dpp is delayed until after Ato patterning begins (Fig 1A,B). This delay suggests that Dpp requires some other signal for its expression in the MF.

To better understand this apparent contradiction, we observed $d p p$-lacZ report in clones that were individually mutant for $a t o, d a$, or the Notch ligands $D l$ and ser. Clones of ato show a cellautonomous loss of $d p p$ report (Fig. 3A). In contrast, $d a$ mutants exhibit strong $d p p$-lacZ activity, nearly WT with the exception that the dorsoventral reporter stripe appears to move more slowly through mutant clones (Fig. 3B). As with Da, canonical Notch signaling is not necessary for $d p p$-lacZ induction, as clones of $D l$ and ser exhibit report, although the lacZ expression is also delayed and weaker with respect to surrounding WT tissue (Fig. 3C). Unexpectedly, Dl and ser mutant tissue showed ectopic dpp-lacZ report at the posterior margin of the eye disc (Fig. 3C, arrows). Further clonal analysis also indicates that Sens is strongly activated in posterior margin cells of mutant eye discs, although Ato was not detected (Fig. 3D, Ato data not shown). It is unlikely that the reporter remains active from its initial induction, but rather reinitiates expression along the posterior margin, as $d p p$-lacZ report is not observed in $\mathrm{Dl}$, ser mutant clones that span between the MF and the posterior margin. Additionally, ectopic posterior margin dpp-lacZ activity is elicited in clusters of cells that are both WT and mutant, suggesting that this effect is the result of non-autonomous signaling that is initiated in mutants (Fig. 3C). The morphogen cycle that drives retinal patterning initiates with the secretion of $\mathrm{Hh}$ at the posterior margin, which then permits the expression of Dpp (Dominguez and Hafen, 1997). Thus, Notch signaling may be required to prevent reinitiation of the MF at the posterior margin of the eye disc.

We next tested to see the effects of removing only repressive Notch signaling, through the loss of either $S u(H)$ or the $E(s p l)$ locus. Discs that bore $S u(H)$ mutants showed diffuse $d p p$ report 
throughout, within both mutant and WT cells (Fig. 3E). In E(spl) mutants, dpp-lacZ reports more similarly to WT, lacking the diffuse expression observed in $S u(H)$ mutants (Fig. 3F). Note the upregulation of $d p p$ report within mutant tissue as compared to WT, a likely reflection of enhanced Ato expression in $E(s p l)$ mutants (Fig. 3F; Ligoxygakis et al., 1998). Thus, the loss of canonical, $\mathrm{Su}(\mathrm{H})$-mediated Notch signaling is not responsible for the re-activation of reporter activity at the posterior margins, and the loss of $E(s p l)$ does not correlate with the diffuse reporter expression observed in $S u(H)$ clones.

In total, the $d p p$-reporter assays indicate that Ato is a necessary component for the induction of $d p p$. Although our observation that $d p p$ reporter induction occurs independent of canonical Notch signaling, it is surprising that Ato appears to function in the absence of its usual partner Da.

\section{Proneural activity provides negative feedback on Hh signaling.}

Previous reports indicate that Dpp requires activation of the Hh signaling response. We examined mutants of MF-associated genes for aberrant Ci processing, under suspicion that ato mutants may lack $d p p$ report due to precocious downregulation of the Hh response. We have monitored Hh response through the buildup of Ciact, the full length form of the Ci transcription factor (Motzny and Holmgren, 1995).

Contrary our expectations, the loss of either Ato or Da results in a continued Hh response; a response that extends into the posterior compartment of the eye disc (Fig. 4A,B). Similarly, removal of Notch ligands also results in continued Ciact accumulation (Fig. 4C), in agreement with reports regarding clones of mutant Notch receptor (Baker and $\mathrm{Yu}, 1997$ ). The reinstitution of $\mathrm{Ci}$ processing in the posterior compartment requires both the specification of neurons and, in particular, Drosophila Epidermal growth factor receptor (DER) signaling (Baker et al., 2009). Both Notch and DER are required for neurogenesis in they eye. Thus, the processes that elicit 
neurogenesis exert negative feedback on Hh signaling. Our result suggests that Ato does not regulate Dpp through feedback on Hh signaling.

As further demonstration of the relationship between retinal neurogenesis and $\mathrm{Ci}$ processing, we look to the Notch pathway transcription factor Suppressor of Hairless $(\mathrm{Su}(\mathrm{H})) . \mathrm{Su}(\mathrm{H})$ mutation elicits a neurogenic phenotype in which most mutant cells commit to the neural R8 fate. Thus, $\mathrm{Su}(\mathrm{H})$ mutants should not display aberrant Ci processing. As expected, Ciact is downregulated within tissue that is mutant for $\mathrm{Su}(\mathrm{H})$ (Fig. 4D, arrows). Additionally, the timing of Ci degradation occurs further anterior in tissue that lack $\mathrm{Su}(\mathrm{H})$ (as well as within $E(s p l)$ clones, Chapter 2). Though untested, it remains possible that the sustained ato-3' observed in ato and da mutants may result from continued Hh response, although similar regulation of $\mathrm{Hh}$ in $\mathrm{Dl}$ and ser clones fail to elicit ato3' upregulation.

\section{RDGN expression is misregulated in MF-specific mutants.}

We next questioned whether sustained ato-3' activity might result from altered regulation of the RDGN. The RDGN has many constituent members, each of which are subject to differential regulation by the signaling pathways used in eye development. We chose to assess the regulation of two members: Ey and Dachshund (Dac). The regulation of these genes differs across several important metrics. Ey is induced independently of Hh and Dpp signaling and its expression dissipates with the passage of the MF. Furthermore, force-expression of Ey within retinal tissue results in ablation of neural fate, and RDGN mutants feature delayed or lost Ato expression and maintenance of Ey to the posterior of the MF (Atkins et al., 2013). Alternatively, Dac is stimulated by both Hh and Dpp. Dac is induced in the pre-proneural region that precedes the MF and is expressed broadly throughout the retinal tissue before ceasing once the MF has progressed by several ommatidial columns. Accordingly observation of Dac can indicate whether cells undergo timely response to Hh and Dpp signaling within mutant tissues. 
In ato or da mutants, Ey expression persists into the posterior portion of the eye disc (Fig. 5A,B). However, the absence of canonical Notch signaling, via the removal of Notch ligands, has no such effect (Fig. 5C). Similarly, $S u(H)$ mutation fails to perturb Ey (Fig. 5D). Thus, inactivation of Ey posterior to the MF is not directly dependent on $\mathrm{Hh}$ signal response, as clones of $\mathrm{Dl}$ and ser display perturbed Ci regulation but WT Ey expression. Furthermore, downregulation of Ey within the posterior compartment is not reliant upon Notch signaling, similar to the regulation of ato-3'.

In either ato, $d a$ or Notch ligand mutant cells, Dac expression persists longer in mutant than in WT tissue (Fig. 5E-G). Additionally, Dac is prematurely lost from cells that are mutant for $\operatorname{Su}(\mathrm{H})$, which perhaps is a reflection of most cells in $\mathrm{Su}(\mathrm{H})$ mutants assuming the neural fate, leaving far fewer undifferentiated cells available to express Dac (Fig. 5H). In total, continued, strong Dac expression into the posterior compartment is, alone, not suggestive of the misexpression of Ato in ato, $d a$ and Notch signaling mutants.

Previous analysis has shown that proper RDGN activity is lost in the absence of Hh and Dpp signaling (Baker and Firth, 2011; Atkins et al., 2013). Additionally, RDGN member Optix is required to coordinate RDGN activity by enabling the Dpp response; without which Ato induction fails to occur (Li et al., 2013). Our observation that Ey downregulation is delayed in ato and da mutant clones suggests the possibility that Ato-Da coordinate the transition from Ey-dependent preproneural RDGN activity to an Ey-independent neurogenic RDGN function.

\section{MF progression is delayed in Notch signaling mutants}

Both Hh and Dpp are required for the proper regulation of MF progression. Dpp provides the long-range inputs that stabilize the formation of the pre-proneural region of the anterior compartment whereas $\mathrm{Hh}$ is received at the posterior margin of the pre-proneural region, eliciting changes in gene expression that coincide with MF formation. It has been suggested that Da has minor, but observable, effects on the movement of the MF, slowing its progress relative to WT tissue (Brown et al., 1996). The MF is demarcated by the apical constriction of the columnar 
epithelia of the eye disc. This constriction can be observed with a variety of markers, including Armadillo (Arm), which accumulates at adherens junctions that form densely within the MF and, in WT are maintained within rosette structures of the posterior compartment (Fig. 6A).

Our analysis of $d a$ clones indicates that MF progression, as detected by Arm immunostaining, is delayed (Fig. 6C) and is in agreement with prior conclusions that were drawn through use of phalloidin immunostain. Clones of either ato or Notch ligands individually produce similar minor delays in MF progression (Fig. 6B,D). Despite delays to MF movement, none of the observed clones featured a delay of more than several cell diameters.

\section{DISCUSSION}

Previous descriptions of MF progression suggest that Hh drives both Ato and Dpp in parallel within the MF. We provide data that augments this model and suggests that Ato is responsible for Dpp expression (Fig. 7A). Though subtle, this difference indicates that Ato is not a passive regulator of MF movement, but an active player. Furthermore, we describe a variety of means through which Ato functions to facilitate the passage of the MF (Fig. 7B-D): through Notch-dependence, Notchindependence, and, though poorly understood, Da-independence.

Ato functions with Notch in the MF to facilitate ato autoregulation (Fig. 7B). In agreement with previous reports, Ato, likely through interaction with $\mathrm{Da}$, elicits Notch signaling. Thus, ato, da and Notch signaling mutants fail to induce 5'-ato activity. It remains unclear whether Ato and Da are actively required to stimulate $5^{\prime}$-ato, or if the induction of Notch signaling alone is sufficient. Although ato is subject to autoregulation, the nature of such autoregulation remains unclear as it has yet to be demonstrated whether or not Ato physically binds to the retina specific enhancer of 5'ato.

Mutants of ato, da and Notch ligands all elicit identical prolonged Hh response. In WT flies, Hh response, as viewed through the accumulation of Ciact, is abrogated in only differentiation photoreceptors. Photoreceptors require a combination of Notch and DER signaling for their 
specification. The loss of either Ato or Da results in severely delayed Notch signaling and near complete loss of neurogenesis (Lim et al., 2008). Thus, the absence of photoreceptors observed in these mutants justifies the continued Hh response.

ato-3' is subject to negative feedback by Ato and Da, but only minimally so by Notch. Report from ato-3' is strongly upregulated in ato and $d a$ mutants, though mutants for both Notch ligands fail to elicit the same effect (Melicharek et al., 2008). This stands in contrast to the activation of 5'ato. The epistasis of these events must occur such that $5^{\prime}$-ato activation either precedes or occurs concurrently with ato-3' cessation. Otherwise, ato autoregulation would be unable to set up with the robustness that is observed in WT. The observation that Notch ligand mutants fail to have the same effect on ato-3' as ato and da, suggests that the downregulation of ato-3' might occur after activation of 5'-ato. Ato and Da function are required to initially stimulate Notch signaling. The earliest phase of Notch signaling drives the expression of $\mathrm{E}(\mathrm{spl})$, which suppresses Ato function and imposes a minor delay on autoregulation. If Notch pathway activity were to actively downregulate ato-3', it would be unlikely that IGs could form. Much of IG formation is solely dependent on ato-3' activity during this early phase of $\mathrm{E}(\mathrm{spl})$ expression. Furthermore, the loss of E(spl) has little, if any, effect on ato-3' report (Chapter 2). If ato-3' is downregulated after the induction of 5'-ato, and ato-3' downregulation is mostly Notch-independent, then a separate signal might regulate the loss of ato-3' report.

The DER pathway is a good candidate for signaling the downregulation of ato-3' (Fig. 7C). Ato stimulates DER through the production and maturation of the DER ligand Spitz (Freeman et al., 1992; Wasserman et al., 2000). Furthermore, Ato, Da, and DER are required for the activation of MAPK in the MF (Chen and Chien, 1999; Lim and Choi, 2004). The activation of MAPK in the MF is debatably required for Ato resolution to R8s, though the mechanism remains elusive (Lesokhin et al., 1999; Yang and Baker, 2001). The use of MAPK to stop ato-3' activity would come well after the 
establishment of Notch signaling in the MF, ensuring that Notch enables strong 5 '-ato activity without restricting coregulators of autoregulation.

We explored the possibility that the change in ato-3' might correspond to modulation of the RDGN with the passage of the MF. Our results are inconclusive, but do indicate that Ey is downregulated in the MF as the result of Ato and Da function, and not that of the Notch pathway. Our data do not exclude the possibility that Ey is downregulated as a consequence of DER-MAPK signaling, though it is not clear if such a relationship would directly impact ato-3' activity. ato-3' is subject to activation through direct binding of Ey in addition to three other RD gene products (Tanaka-Matakatsu et al., 2014). This result further stresses that the dynamic rewiring of the RDGN that occurs within the MF is still poorly understood. The relationship between the RDGN, Notch, and DER signaling represents a great void in our understanding of how this gene expression network becomes altered with the passage of the MF.

The secretion of Dpp from cells of the MF is a critical component of MF progression. The classical mutants $R o^{D}$ and $\operatorname{Bar}^{1}$ feature the prominent furrow-stop phenotype (Chanut et al., 2000; Kojima et al., 1991). Furrow-stop mutants feature an eye with an anterior cleft and severe loss of the overall eye field. Studies of these mutants have provided much of the mechanism of MF movement. However, the cyclical nature this genetic program has failed to accurately describe a role for Ato. Retinogenesis requires Ato, without which photoreceptors cannot be specified (Jarman et al., 1994). However, Ato is not just a passive member in Hh-Dpp cycling. Our findings suggest that Ato is required within the MF for the production of Dpp Past efforts have indicated that the dominant rough (ro) allele $R o^{D}$, non-autonomously restricts MF movement. However, Ro is a repressor that has thus far only been implicated in the repression of Ato and its target Sens. If $R o^{D}$ non-autonomously suppresses MF progress through either Ato or Sens, this would suggest that either Ato or Sens regulate the induction of a signal that is required for MF movement. Such is the case for Ato and its regulation of Dpp. To rule out the possibility that Ro might directly regulate 
Dpp, we assessed $d p p$-lacZ report in clones of mutant $r o$, and observed no expansion of dpp report (data not shown).

Class II bHLHs like Ato are thought to function solely through heterodimerization with class I bHLHs. Da is the only predicted class I bHLH in the D. mel. genome. Our $d p p$-lacZ assays hint at Ato function in the absence of Da (Fig. 7D). We considered the possibility that loss of Ato results in aberrant patterning of other MF-related genes, eliciting loss of Dpp. However, previous studies with the ato $^{1}$ mutant reveal the presence of Da within the MF at marginally higher levels, and nearly WT Emc expression. Thus, Da is present in the ato mutant MF and removal of Da fails to significantly impact $d p p$ reporter activity. We conclude that Da is not cell-autonomously required for Dpp induction.

As for Ato function, we speculate that this novel mechanism requires DNA-binding, as with known Ato-Da function. ato ${ }^{1}$ differs from its WT allele by three point mutations, two in the basic domain and a third near the N-terminus (Jarman et al., 1994). The basic mutations likely ablate DNA-binding, but may perturb little else with regard to Ato interactivity and functionality, hence the ability to detect Ato protein in $a t o^{1}$ mutants via immunostaining procedures. As reported here, clones of $a t^{1}$ exhibit high-intensity ato-3' report, in agreement with the high level of Ato labeling that is observed in such mutants. We speculate that if Ato were stimulating $d p p$-lacZ through a DNA-independent capacity, these high-level Ato clones would be likely to elicit $d p p$ report, contrary to our observations. Likewise, our analysis precludes a role for canonical Notch signaling in Dpp induction, as such a role would require both Da and Notch ligands within the MF. Removal of either fails to prevent $d p p$ report.

In total, the presented findings demonstrate that Ato is proactive in the maintenance of both the anterior and posterior compartments; the former through the induction of Dpp, and the latter through the formation of neurons that secrete Hh. Importantly, Dpp induction requires a novel, asof-yet undefined mechanism of Ato function that is independent of Da. 


\section{MATERIALS AND METHODS}

\section{Drosophila genetics}

Flies were cultured on yeast-dextrose media at $24^{\circ} \mathrm{C}$ and maintained according to a typical diurnal schedule. ato ${ }^{1}$ encodes A25T, K253N and N261I, the last of which putatively disrupts DNA binding (Jarman et al., 1994). $D l^{\text {RevF10 }}$ is the result of an imprecise P-element excision that has removed the enhancer, promoter and transcription start site (Ligoxygakis et al., 1998). Ser ${ }^{R X 82}$ features the deletion and disruption of two 5' exons (Ligoxygakis et al., 1998). emc ${ }^{A P 6}$ is a $400 \mathrm{bp}$ deletion of the emc locus; mutants lack antigenicity for commonly used $\alpha$-Emc antibodies (Ellis, 1994; Bhattacharya and Baker, 2012). $d a^{10}$ results from a $4 \mathrm{~kb}$ deletion; mutants lack antigenicity for commonly used $\alpha$-Da antibodies (Caudy et al., 1988; Lim and Choi, 2008). $S u(H)^{\wedge 47}$ results from an imprecise P-element excision that removes transcription start sites of $S u(H)$ and $l(2) B g 35$ (Morel and Schweisguth, 2000). The rescue construct $P\{l(2) B g 35+\}$ obviates contributions of $l(2) B g 35$ to Su(H) loss-of-function phenotypes described herein (Morel and Schweisguth, 2000). Similarly, $D f(3 R) E(s p l)^{b 32.2}$ removes the entire $E(s p l)$ locus, including gro (Schrons et al., 1992). gro is rescued by the inclusion of $P\{g r o+\}$ (Heitzler et al., 1996). $r 0^{x 63}$ harbors a deletion in the $5^{\prime}$ coding region that results in frameshift after 30aa (Kimmel et al., 1990). Reporter stocks used are as follows: $P\left\{w^{+m C}\right.$ ato5'F:9.3\} and $P\left\{w^{+m C}\right.$ ato3'F:5.8\} (Sun et al., 1998); $P\{B S 3.0$ dpp-lacZ\} (Blackman et al., 1991).

\section{Immunohistochemistry}

All steps were performed at room temperature unless otherwise indicated. Tissues were dissected in $0.1 \mathrm{M}$ sodium phosphate buffer and fixed in $4-6 \%$ formaldehyde, $0.1 \mathrm{M}$ sodium phosphate buffer. Tissues were washed in $0.3 \%$ Trition, $0.1 \mathrm{M}$ sodium phosphate buffer bathed in primary antibody mixtures (antibody concentrations shown below in 1\% BSA, 0.1M sodium phosphate buffer) for $12-18$ hours at $4^{\circ} \mathrm{C}$. Primary antibodies were removed, tissues were washed in $1 \%$ BSA in $0.1 \mathrm{M}$ sodium phosphate buffer and bathed in secondary antibody mixtures $11: 1000$ 
dilution for each secondary, in 1\% BSA, $0.1 \mathrm{M}$ sodium phosphate buffer) for $2 \mathrm{hrs}$. Secondary antibody mixtures were removed, tissues were washed $0.1 \mathrm{M}$ sodium phosphate buffer. Tissues were mounted in 60\% glycerol and imaged using an Olympus Fluoview FV1000 Confocal microscope. All scanning data reported was observed in a minimum of tissues from 5 independent animals of like genotype.

Primary antibodies include mouse rabbit $\alpha$-Ato (1:5000, Jarman et al., 1994); rat $\alpha$-Ciact-2A1 (1:100, Motzny and Holmgren, 1995); mouse $\alpha$-Ey (1:100, Clements et al., 2008); mouse $\alpha$ - $\beta$-gal-401a (1:800-1000, Ghattas et al., 1991); mouse $\alpha$-E(spl)-mAb323 (1:3, Jennings et al., 1994); guinea pig $\alpha$-Sens (1:500-800, Nolo et al., 2000); mouse $\alpha$-Dac2-3 (1:100, Mardon et al., 1994); and mouse $\alpha$-Arm (1:100, Riggleman et al., 1990). Rat $\alpha$-Ciact-2A1, mouse $\alpha$-Ey, mouse $\alpha$ - $\beta$-gal-40-1a, mouse $\alpha$ Dac2-3 and mouse $\alpha$-Arm were obtained from the Developmental Studies Hybridoma Bank, created by the NICHD of the NIH and maintained at The University of Iowa, Department of Biology, Iowa City, IA 52242. Tissues to be labeled with primary rabbit $\alpha$-Ato were dissected in $0.3 \%$ Triton, $0.1 \mathrm{M}$ sodium phosphate buffer.

Secondary antibodies used include 488-goat $\alpha$-rat (Life Technologies), 488-goat $\alpha$-mouse (Jackson), 488-rabbit $\alpha$-GFP (Life Technologies), 546-goat $\alpha$-mouse (Life Technologies), 546-goat $\alpha$ Rat (Life Technologies), 633-goat $\alpha$-guinea pig (Life Technologies), 633-goat $\alpha$-rabbit (Life Technologies) and 633-goat $\alpha$-mouse (Life Technologies).

\section{Image Production}

All images were processed in Adobe Photoshop CC v. 14.2. Image manipulations of brightness/contrast and color balance were applied uniformly across each image shown. Images were then collated in Adobe Illustrator CC v. 17.1 before being exported to Microsoft Word v. 14.4.4. 


\section{ACKNOWLEDGEMENTS}

We are grateful to Yuh Nung Jan, Nick Baker, Graeme Mardon, Sarah Bray, Hugo Bellen and Andrew Dacks for their generous gifts of flies and antibodies, without which this work could not have been accomplished. 

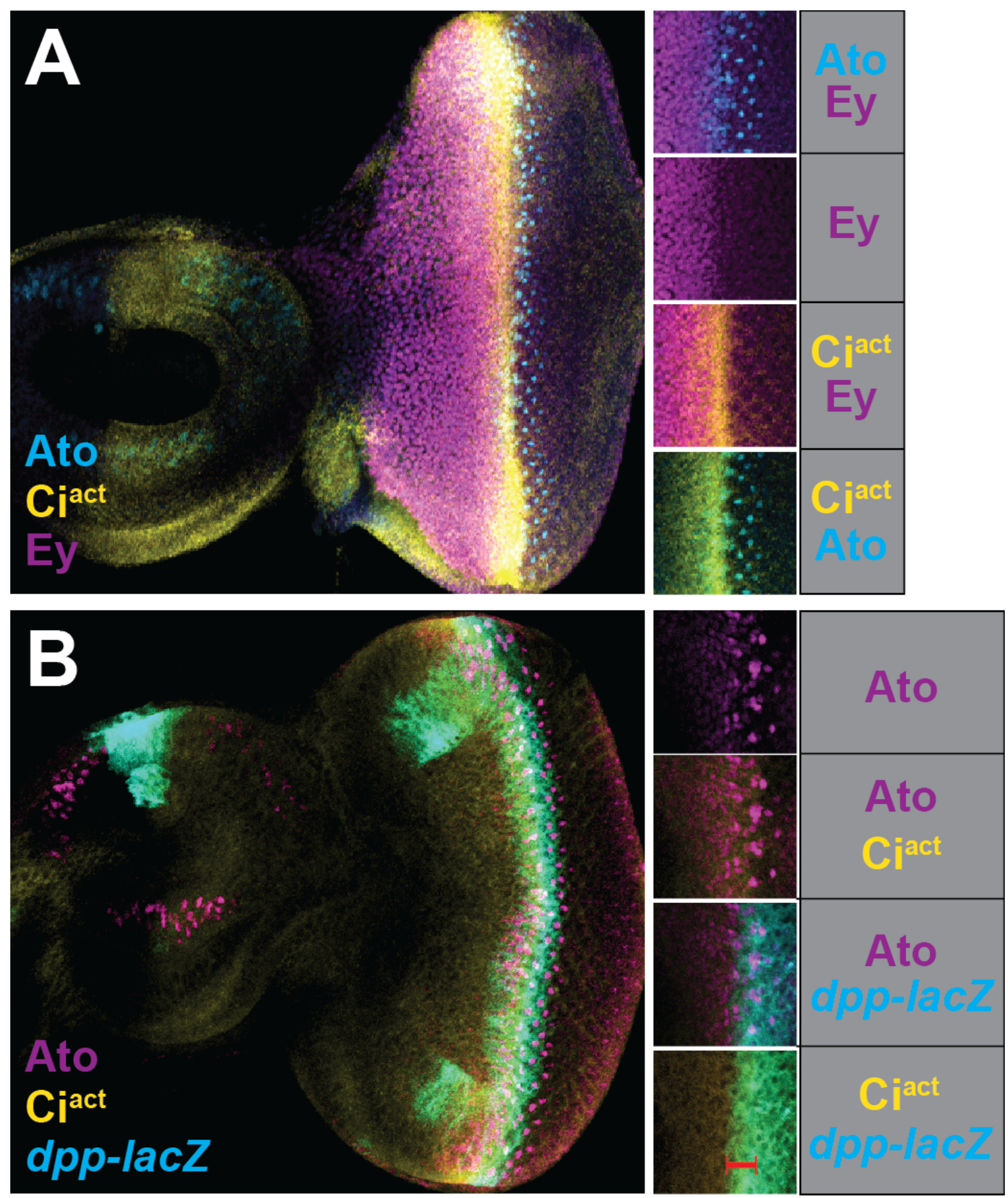

Fig. 1. Ato precedes induction of dpp-lacZ and downregulation of Ey. (A) Anterior is the left, as in all figures. In the MF, Ato is patterned into IGs as Ey expression ebbs to background levels. Ciact accumulation peaks in cells that are just posterior to those that cease high level Ey expression. (B) Ato precedes $d p p$-lacZ activity. Insets at right feature a small subset of confocal sections shown to the left. Sections reveal that $d p p-l a c Z$ is not expressed until Ato begins to be refined into isolated clusters. Ciact is maintained at high levels through the first several cell diameters of $d p p$-lacZ report, as denoted by the bracket. Genotypes: $w^{1118}$. 


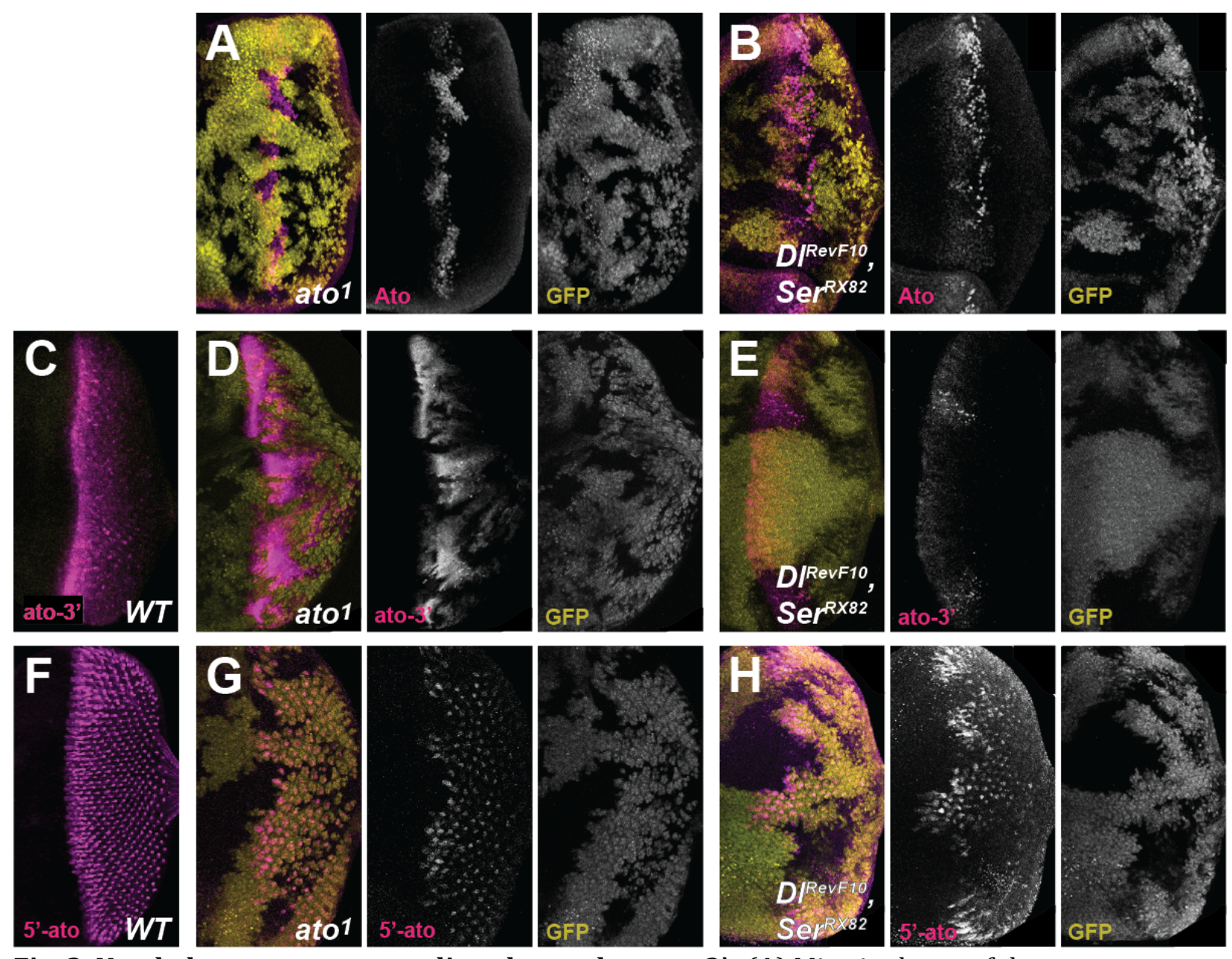

Fig. 2. Notch does not appear to directly regulate ato-3'. (A) Mitotic clones of the mutant genotype indicated; GFP marks WT tissue, the absence of GFP indicates homozygous mutant cells. Ato1 mutants produce a nonfunctional Ato that maintains antigenicity. In ato mutants, ato is expressed more strongly and without patterning that is characteristic of WT. (B) Dl and Ser clones exhibit high level Ato, but only in cells that are anteriorly adjacent to WT tissue. Larger mutant regions lack strong Ato expression, though low-levels remain detectable. (C) ato-3'-lacZ reporter is active in the MF. Due to $\beta$-gal perdurance, report progressively dissipates throughout the posterior of the eye. (D) ato-3' report is strongly upregulated in ato mutant clones. (E) Dl and Ser clones have much lesser effect on ato-3' than seen in ato mutants. (F) 5'-ato-lacZ reports in IGs and nascent R8s, though perdurance of B-gal allows continued staining of R8s throughout the posterior. (G) Ato is cell autonomously required for the activation of 5-ato. (H) Dl and Ser are also required for 5'-ato activity. Some 5'-ato activity can be detected within mutant cells, but only adjacent to WT tissues, indicating that cells require reception of the Notch signal to activate 5 '-ato. Genotypes: (A)

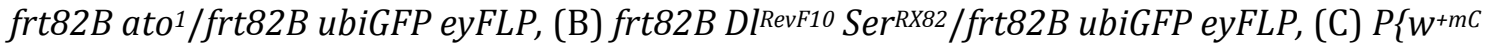
ato3'F:5.8\}/+, (D) $P\left\{w^{+m C}\right.$ ato3'F:5.8\}/+;frt82B ato $1 /$ frt82B ubiGFP eyFLP, (E) $P\left\{w^{+m C}\right.$ ato3'F:5.8\}/+; frt82B DIRevF10 Ser ${ }^{R X 82} /$ frt82B ubiGFP eyFLP, (F) $P\left\{w^{+m C}\right.$ ato5' $\left.F: 9.3\right\} /+$, (G) $P\left\{w^{+m C}\right.$ ato $\left.5^{\prime} F: 9.3\right\} /+$; frt82B ato $^{1} /$ frt82B ubiGFP eyFLP $(\mathrm{H}) P\left\{w^{+m C}\right.$ ato5'F:9.3\}/+; frt82B DlRevF10 $\operatorname{Ser}^{R X 82} /$ frt82B ubiGFP eyFLP. 

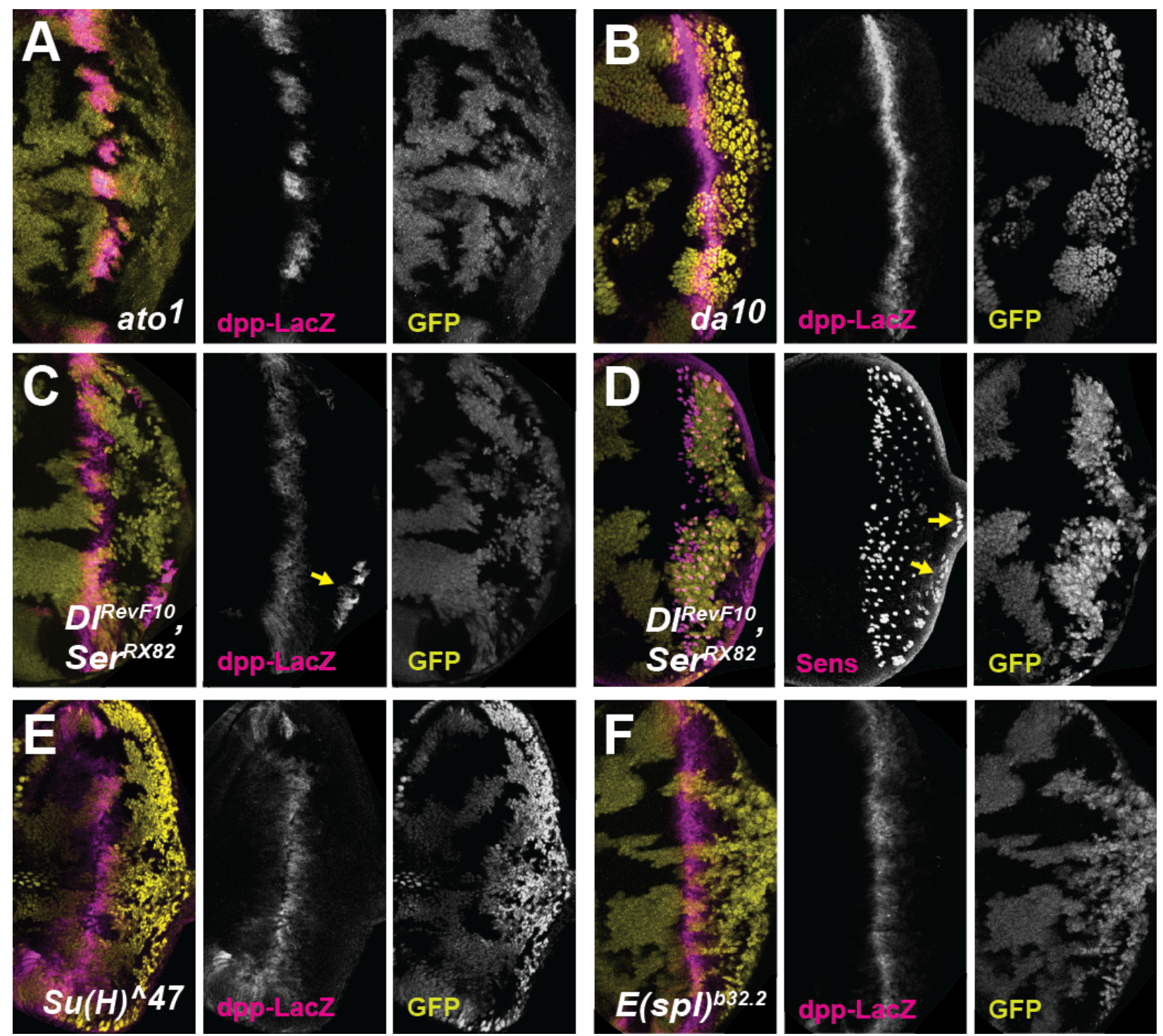

Fig. 3. Ato mutants fail to activate $\boldsymbol{d p p}$-IacZ. (A) ato cell-autonomously regulates $d p p$-lacZ report. $(\mathbf{B}, \mathbf{C}) d a$ and Notch ligand mutants exhibit $d p p$-lacZ activity. However, $d p p$-lacZ is perturbed in $D l$, ser dual mutants, as $d p p$ report is slightly delayed in mutants and ectopically activated at the posterior margins (arrow). At posterior margins, $d p p$-lacZ appears to non-autonomous, as both both mutants and WT cells stain for $\beta$-gal. (D) $D l$, ser mutants also exhibit upregulated Sens along the posterior margin (arrows). (E) $S u(H)$ mosaic discs elicit a broadening of the normally narrow $d p p$-lacZ stripe. (F) Removal of the $E$ (spl) locus exhibits less severe defects than the loss of $\mathrm{Su}(\mathrm{H})$, with local upregulation of $d p p$-lacZ, but no otherwise aberrant patterning. Genotypes: (A) $P\{B S 3.0$ dpp-lacZ\}/+;frt82B ato $/$ frt82B ubiGFP eyFLP, (B) da10 frt40A/ubiGFPnls frt40A;P $\{B S 3.0 \mathrm{dpp}$ lacZ\}/eyFLP, (C) P $\{B S 3.0$ dpp-lacZ\}/+;frt82B DlRevF10 SerRX82/frt82B ubiGFP eyFLP, (D) frt82B DIRevF10 SerRX82/frt82B ubiGFP eyFLP, (E) Su(H)^47 frt40A P\{l(2)35bg+ $\} /$ ubiGFPnls frt40A; $P\{B S 3.0$ dpplacZ\}/eyFLP; (F) P\{BS3.0 dpp-lacZ $\} /+; f r t 82 B$ Df(3R)E(spl)b32.2 P\{gro+\}/frt82B ubiGFP eyFLP. 

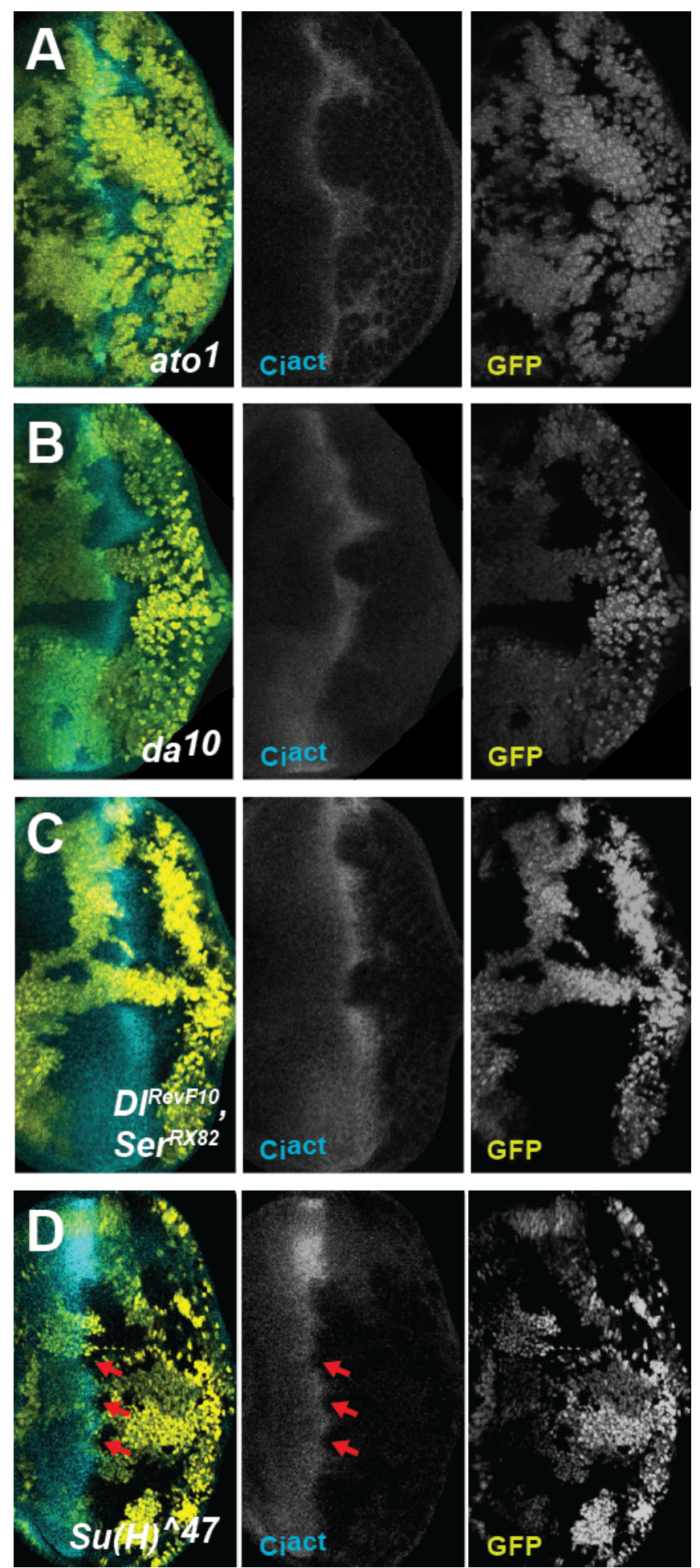

Fig. 4. Notch signaling prevents prolonged Ciact accumulation. (A-C) Tissues mutant for either ato, da, or Notch ligands all exhibit prolonged accumulation of Ciact into the posterior compartment. (D) Alternately, $\mathrm{Su}(\mathrm{H})$ mutants exhibit precocious loss of Ciact when compared to

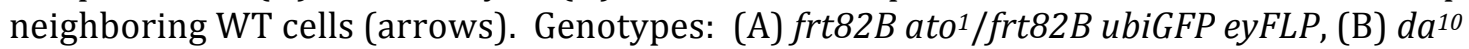
frt40A/ubiGFPnls frt40A;eyFLP/+; (C) frt82B DIRevF10 SerRX82/frt82B ubiGFP eyFLP, (D) Su(H)^47 frt40A $P\{l(2) B g 35+\} /$ ubiGFPnls frt40A; eyFLP/+. 

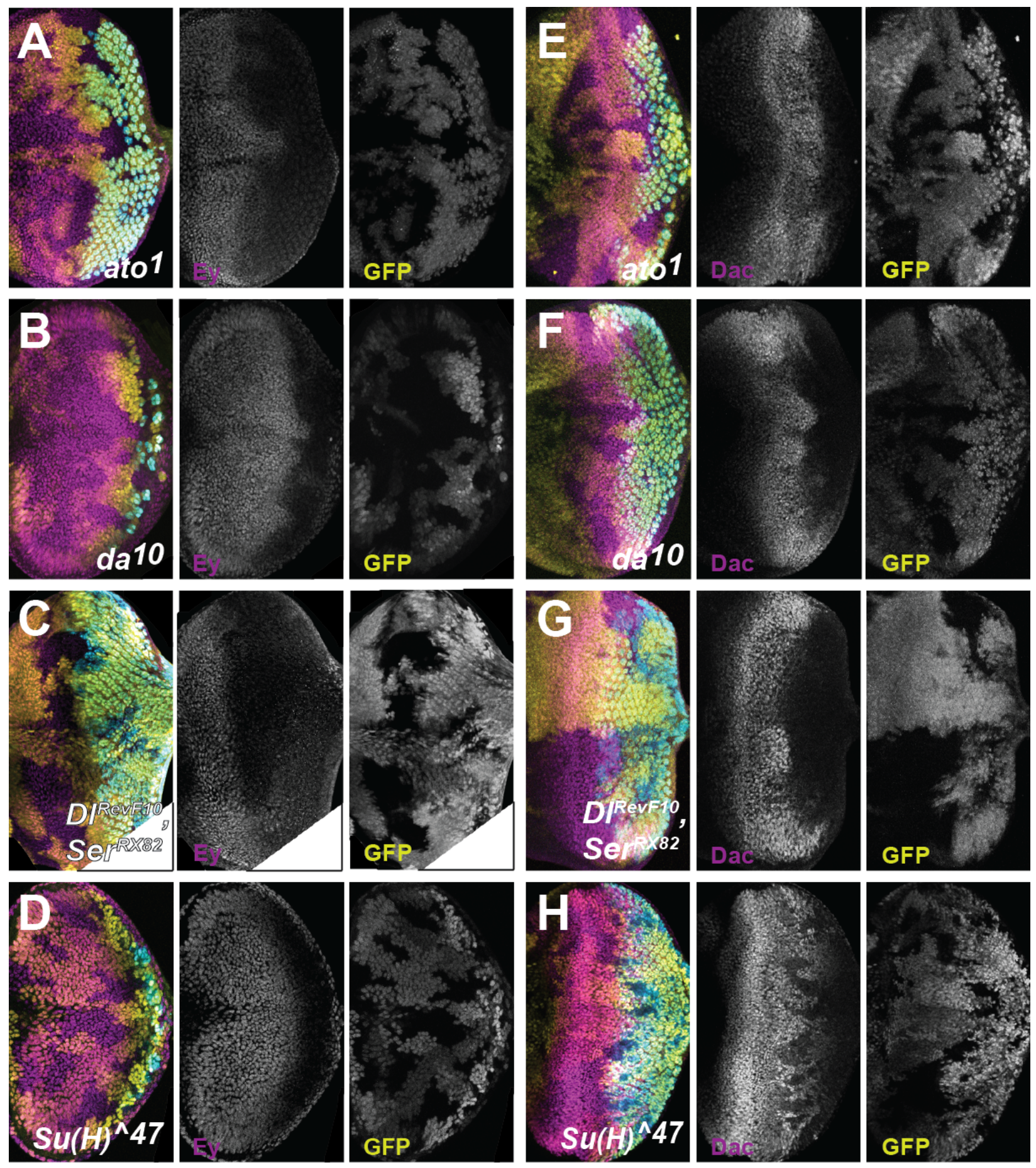

Fig. 5. Ato and Da negatively regulate Ey in the MF. (A,B) Ey fails to downregulate in ato and $d a$ mutants with the passage of the MF. (C) However, Notch signaling mutants $(\mathrm{Dl}$, ser or $\mathrm{Su}(\mathrm{H})$ ) exhibit no such defect and Ey appears to lose high-level expression as in WT. (E-G) Dac expression is enhanced in the posterior compartment of ato, $d a$, and Notch ligand mutants. (H) $S u(H)$ mutants elicit the opposite effect with early downregulation of Dac as compared to WT cells. Genotypes:

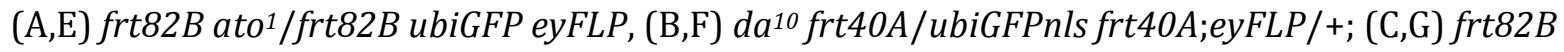
DlRevF10 Ser ${ }^{\text {RX82 } / f r t 82 B ~ u b i G F P ~ e y F L P, ~(D, H) ~ S u(H) ~}{ }^{\wedge 47}$ frt40A P\{l(2)Bg35+\}/ubiGFPnls frt40A;eyFLP/+. 

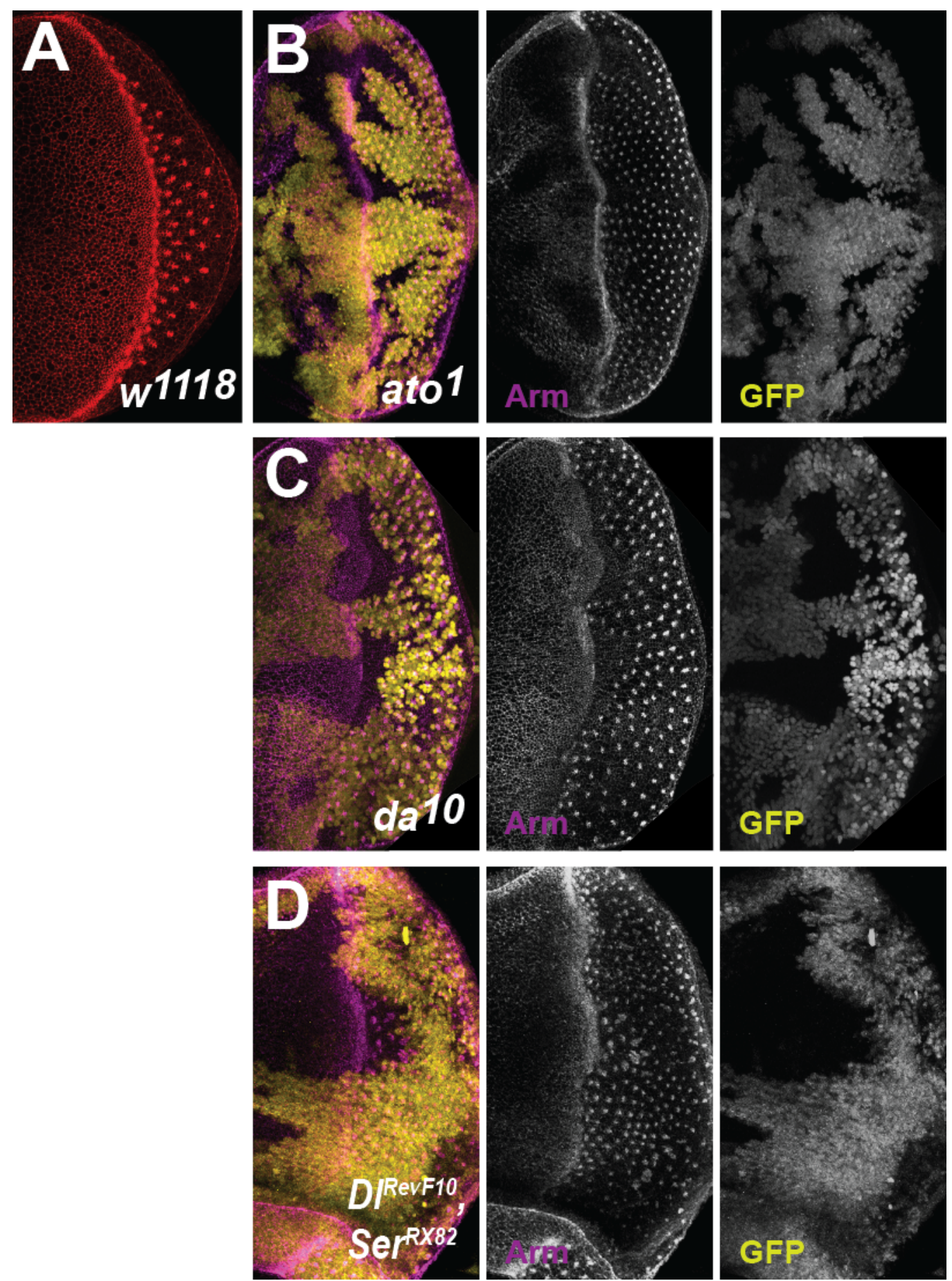

Fig. 6. MF progression is slowed through proneural mutants. (A) Arm is expressed throughout all developing tissues, where it accumulates at ahderens junctions. Apical constriction within the MF causes a concentration of Arm in a dorsoventral band. Arm remains at high levels in rosette structures throughout the posterior compartment. (B) ato mutants elicit slowed MF progression, as determined by a lagging dorsoventral Arm staining in such mutants. (C,D) Similarly, both $d a$ and Notch ligand mutants feature slowed MF progress seen in ato. Genotypes: (A) frt82B ato $1 /$ frt82B $^{2}$ ubiGFP eyFLP, (B) da ${ }^{10}$ frt40A/ubiGFPnls frt40A;eyFLP/+; (C) frt82B DlRevF10 SerRX82/frt82B ubiGFP eyFLP. 

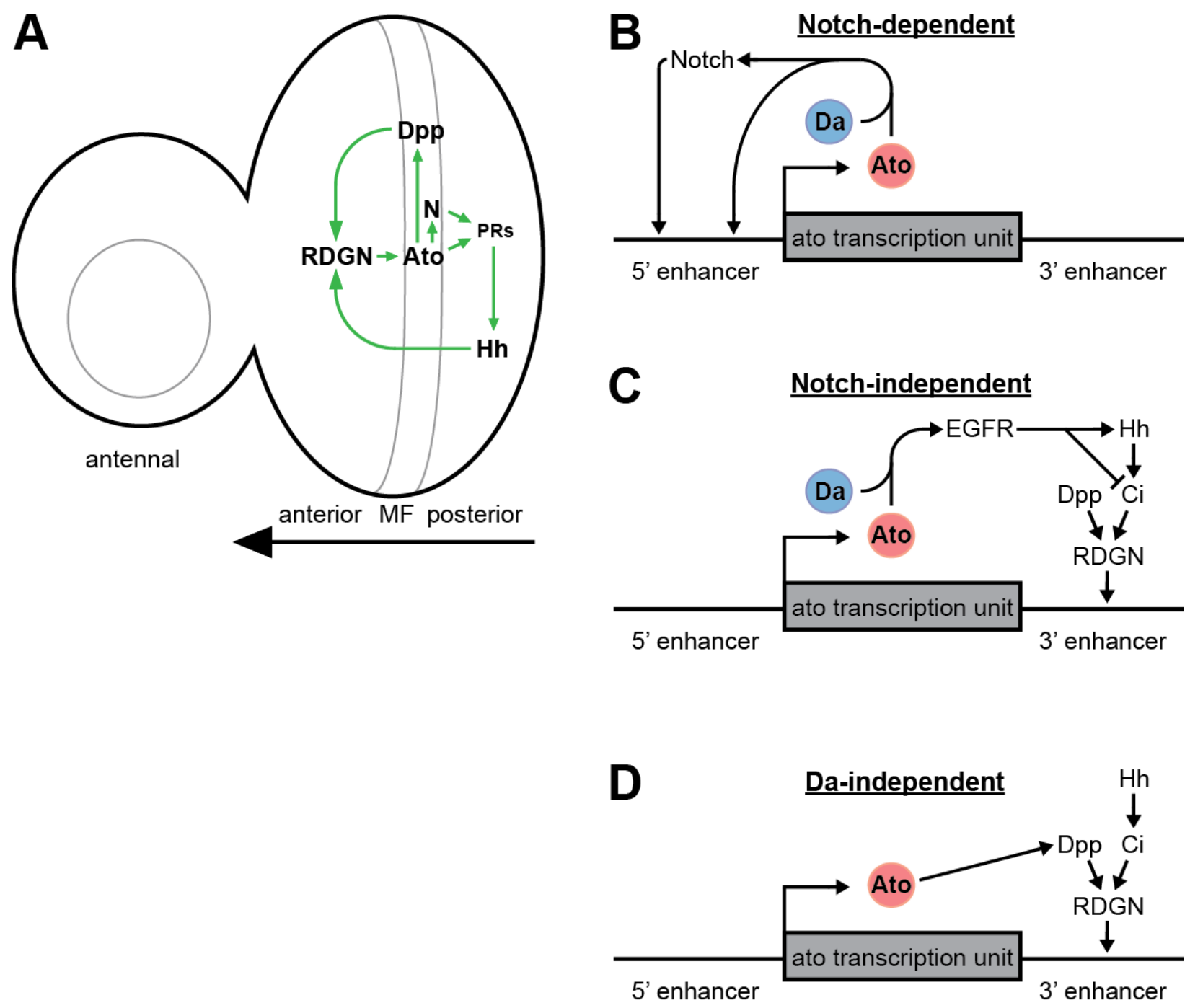

Fig. 7. Ato confers both anterior and posterior compartmental identity through independent mechanisms. (A) Ato participates in morphogen cycling through direct genetic interaction with dpp. Thus, Hh drives Ato, which, in turn, facilitates dpp activity, as assayed by the dpp-lacZ reporter. (B) Ato, in conjunction with $\mathrm{Da}$, permits Notch signaling which is required for ato autoregulation. (C) Independently of Notch, Ato and Da are required to promptly downregulate ato-3'. We propose that this Notch-independent function may occur through DER signaling, as DER activation effects both the Hh pathway and RDGN gene function. (D) Lastly, Ato functions independently of Da in activating the expression of dpp-lacZ. This may constitute a novel function for a class II bHLH. 


\section{FUTURE PERSPECTIVES}

In this work we have investigated the nature of Notch signaling during Drosophila retinogenesis. In doing so, we have revealed that Notch biphasicity is not a straight-forward two step process, but something more complex. The inductive phase of Notch does not require Aop in the absence of E(spl). Hence, $\mathrm{Su}(\mathrm{H})$ mutants, in which Aop and E(spl) are lost, elicit R8 specification. However, the $\mathrm{Su}(\mathrm{H})$ components are required for healthy retinogenesis in the sense that the specification of R8s is of no use without their proper spacing from each other. Aop allows the time for this spacing mechanism to set in.

Moving forward, the biggest question will be to determine how DER-MAPK plays into this Aop-dependent Notch mechanism. Chapter 3, Fig. 7 examines the role of the repressor Ro. We were able to show that Aop regulates ro, preventing its expression within the MF. Evidence for this interaction had been mounting, as Ro is absent from DER mutants, and two genomic studies have revealed that Aop likely binds to the ro enhancer (Dominguez, 1997; Webber et al., 2013; Potier et al, 2014). However, the loss of both aop and ro failed to rescue the Ato phenotype observed in aop alone. Despite this, ro loss-of-function rescues the interaction of $a \mathrm{op}^{1} / \mathrm{Der}^{E l p}$. A reasonable solution to both data might be to suggest that $D E R^{E l p}$ elicits precocious MAPK activation, which would permit a variety of MAPK-dependent functions in the MF. In aop, ro dual mutants, MAPK may not be active in the MF, effectively isolating the Ro gain-of-function phenotype of the aop mutants. This suggests that Aop is not protecting Ato from basal MAPK activation, but that Aop has a dedicated separate function. Despite our limited analysis of Bar and Roe, the specific mechanism of Aop function is yet to be found. For now, we must be content in knowing that Aop promotes ato-3' activity.

Another point of emphasis is the role of Roe. Its expression is guided by Notch signaling, yet its activity appears to initiate after other major Notch-dependent processes have begun (del Alamo and Mlodzik, 2008). A full analysis of Roe is in order, to determine 1) why its activity is delayed, 2) what causes its activity to quickly decline. We hypothesized that MAPK, whose activation coincides 
with the end of Roe activity, might posttranslationally regulate Roe (Chen and Chien, 1999; Melicharek et al, 2008). Upon looking for putative MAPK consensus motifs, P-X-S/T-P, none were found (though Roe's paralog, Rn, has a prominent, conserved MAPK site). Despite our current lack of leads on this project, obtaining a better understanding of Roe will provide a more detailed landscape of Ato autoregulation and potential interactions between Notch and other signals in the MF.

A final point of interest is in understanding how ato-3' ceases function. Data are clear that removal of Aop elicits premature loss of ato-3' and aborted IG formation. Interestingly, ato-3' is negatively regulated by Ato and Da, but not by Notch (at least not to nearly the same degree). This suggests that Ato and Da are activating a signal or gene other than Notch to accomplish this function. It is important to note that the activation of MAPK is cell-autonomously regulated by Ato and Da (Chen and Chien, 1999). This is interesting because one would assume that ato and da mutants could respond to DER ligand secreted from other cells, though this is not the case. Though somewhat speculative at this point, we propose that Ato and Da regulate the ability of cells in the MF to respond to MAPK for the purpose of stopping expression from ato-3'. Ceasing ato-3' activity is the real key to repressing ato. MAPK could stop expression from ato-3' in a variety of ways, potentially through effects on the RDGN, or perhaps through the activation or induction of specific repressors. As no new repressors of Ato have identified in the past decade, we suggest that future efforts be directed toward either the RDGN and/or Lilliputian, the FMR2 ortholog (Distefano et al., 2012). 


\section{REFERENCES}

Acar M, Jafar-Nejad H, Takeuchi H, Rajan A, Ibrani D, Rana NA, Pan H, Haltiwanger RS, Bellen HJ. (2008). Rumi Is a CAP10 Domain Glycosyltransferase that Modifies Notch and Is Required for Notch Signaling. Cell 132, 247-58.

Alifragis P, Poortinga G, Parkhurst SM, Delidakis C. (1997). A network of interacting transcriptional regulators involved in Drosophila neural fate specification revealed by the yeast two-hybrid system. Proc. Natl. Acad. Sci. 94, 13099-104.

Alvaredo D, Klein DE, Lemmon MA. (2010). Structural basis for negative cooperativity in growth factor binding to an EGF receptor. Cell 142, 568-79.

Andersson ER, Lendahl U. (2014). Therapeutic modulation of Notch signalling - are we there yet? Nat. Rev. Drug Disc. 13, 357-78.

Atkins M, Jiang Y, Sansores-Garcia L, Jusiak B, Halder G, Mardon G. (2013). Dynamic Rewiring of the Drosophila Retinal Determination Network Switches Its Function form Selector to Differentiation. PLOS Genetics 9, e1003731.

Aza-Blanc P, Ramirez-Weber FA; Laget MP, Schwartz C, Kornberg TB. (1997). Proteolysis That Is Inhibited by Hedgehog Targets Cubitus interruptus Protein to the Nucleus and Converts It to a Repressor. Cell 89, 1043-53.

Bailey AM, Posakony JW. (1995). Suppressor of hairless directly activates transcription of enhancer of split complex genes in response to Notch receptor activity. Genes Dev. 9, 2609-22.

Baker NE, Rubin GM. (1992). Ellipse mutations in the Drosophila homologue of the EGF Receptor affect pattern formation, cell division and cell death in eye imaginal discs. Dev. Biol. 150, 381-96.

Baker NE, Yu S, Han D. (1996). Evolution of proneural atonal expression during distinct regulatory phases in the developing Drosophila eye. Curr. Biol. 6, 1290-301.

Baker NE, Yu S-Y. (1997). Proneural function of neurogenic genes in the developing Drosophila eye. Curr. Biol. 7, 122-32.

Baker NE, Bhattacharya A, Firth LC. (2009). Regulation of Hh signal transduction as Drosophila eye differentiation progresses. Dev. Biol. 335, 356-66.

Baker NE, Firth LC. (2011). Retinal determination genes function along with cell-cell signals to regulate Drosophila eye development. Bioessays 33, 538-46.

Baker RH, Kuehl JV, Wilkinson GS. (2011). The Enhancer of split complex arose prior to the diversification of schizophoran flies and is strongly conserved between Drosophila and stalk-eyed flies. (Diopsidae). BMC Evo. Biol. 11, 354.

Baonza A, Freeman M. (2001). Notch signalling and the initiation of neural development in the Drosophila eye. Development 128, 3889-98. 
Barolo S, Stone T, Bang AG, Posakony JW. (2002). Default repression and Notch signaling: Hairless acts as an adaptor to recruit the corepressors Groucho and dCtBP to Suppressor of Hairless. Genes Dev. 16, 1964-76.

Berman BP, Pfeiffer BD, Laverty TR, Salzberg SL, Rubin GM, Eisen MB, Celniker SE. (2004). Computational identification of developmental enhancers: conservation and function of transcription factor binding-site clusters in Drosophila melanogaster and Drosophila pseudoobscura. Genome Biol. 5, R61.

Berry LW, Westlund B, Schedl B. (1997). Germ-line tumor formation caused by activation of $g l p-1$, a Caenorhabditis elegans member of the Notch family of receptors. Development 124, 925-36.

Bessa J, Gebelein B, Pichaud F, Casares F, Mann RS. (2002). Combinatorial control of Drosophila eye development by Eyeless, Homothorax, and Teashirt. Genes Dev. 16, 2415-27.

Bhattacharya A, Baker NE. (2009). The HLH protein Extramacrochaetae is required for R7 cell and cone cell fates in the Drosophila eye. Dev. Biol. 327, 288-300.

Bhattacharya A, Baker NE. (2011). A Network of Broadly Expressed HLH Genes Regulates TissueSpecific Cell Fates. Cell 147, 881-92.

Bhattacharya A, Baker NE. (2012). The Role of the bHLH Protein Hairy in Morphogenetic Furrow Progression in the Developing Drosophila Eye. PLOS One 7, e47503.

Blackman RK, Sanicola M, Raftery LA, Gillevet T, Gelbart WM. (1991). An extensive 3' cisregulatory region directs the imaginal disk expression of decapentaplegic, a member of the TGF- $\beta$ family in Drosophila. Development 611, 657-65.

Boislcair Lachance JF, Pelaez N, Cassidy JJ, Webber JL, Rebay I, Carthew RW. (2014). A comparative study of Pointed and Yan expression reveals new complexity to the transcriptional networks downstream of receptor tyrosine kinase signaling. Dev. Biol. 385, 263-78.

Borod ER, Heberlein U. (1998). Mutual regulation of decapentaplegic and hedgehog during the initiation of differentiation in the Drosophila retina. Dev. Biol. 197, 187-197.

Bose A, Kahali B, Zhang S, Lin J-M, Allada R, Karandikar U, Bidwai AP. (2006). Drosophila CK2 regulates lateral-inhibition during eye and bristle development. Mech. of Dev. 123, 649-64.

Bose A, Majot AT, Bidwai AP. (2014). The Ser/Thr Phosphatase PP2A Regulatory Subunit Widerborst Inhibits Notch Signaling. PLOS One 9, e101884.

Brand M, Campos-Ortega JA. (1990). Second-site modifiers of the split mutation of Notch define genes involved in neurogenesis in Drosophila melanogaster. Roux's Arch. Dev. Biol. 198, 275-85.

Brown NL, Sattler CA, Markey DR, Carroll SB. (1991). hairy gene function in the Drosophila eye: normal expression is dispensable but ectopic expression alters cell fates. Development 113, 124556. 
Brown NL, Sattler CA, Paddock SW, Carroll SB. (1995). Hairy and Emc Negatively Regulate Morphogenetic Furrow Progression in the Drosophila Eye. Cell 80, 879-87.

Brown NL, Paddock SW, Sattler CA, Cronmiller C, Thomas BJ, Carroll SB. (1996). daughterless is required for Drosophila photoreceptor cell determination, eye morphogenesis, and cell cycle progression. Dev. Biol. 179, 65-78.

Bruckner K, Perez L, Clausen H, Cohen S. (2000). Glycosyltransferase activity of Fringe modulates Notch-Delta interactions. Nature 406, 411-5.

Brummel T, Twombly V, Marques G, Wrana JL, Newfeld SJ, Attisano L, Massague J, O'Connor MB, Gelbart WM. (1994). Characterization and relationship of dpp receptors encoded by the saxophone and thick veins genes in Drosophila. Cell 78, 251-61.

Burke R, Basler K. (1996). Hedgehog-Dependent Patterning in the Drosophila Eye Can Occur in the Absence of Dpp Signaling. Dev. Biol. 179, 360-8.

Cagan RL, Ready DF. (1989). Notch is required for successive cell decisions in the developing Drosophila retina. Genes Dev. 3, 1099-1112.

Caudy M, Vassin H, Brand M, Tuma R, Jan LY, Jan YN. (1988). daughterless, a Drosophila gene essential for both neurogenesis and sex determination, has sequence similarities to myc and the achaete-scute complex. Cell 55, 1061-7.

Cave JW, Loh F, Surpris JW, Xia L, Caudy MA. (2005). A DNA Transcription Code for Cell-Specific Gene Activation by Notch Signaling. Curr. Biol. 15, 94-104.

Caviglia S, Luschnig S. (2013). The ETS domain transcriptional repressor Anterior open inhibits MAP kinase and Wingless signaling to couple tracheal cell fate with branch identity. Development 140, 1240-9.

Chanut F, Luk A, Heberlein U. (2000). A Screen for Dominant Modifiers of ro ${ }^{\text {Dom }}$, a Mutation That Disprupts Morphogenetic Furrow Progression in Drosophila, Identifies Groucho and Hairless as Regulators of atonal Expression. Genetics 156, 1203-17.

Chen CH, von Kessler DP, Park W, Wang B, Ma Y, Beachy PA. (1999). Nuclear Trafficking of Cubitus interruptus in the Transcriptional Regulation of Hedgehog Target Gene Expression. Cell 98, 305-16.

Chen C-K, Chien C-T. (1999). Negative regulation of atonal in proneural cluster formation of Drosophila R8 photoreceptors. Proc. Natl. Acad. Sci. 96, 5055-60.

Chen RH, Derynck R. (1994). Homomeric interactions between type II transforming growth factorbeta receptors. J. Biol. Chem. 269, 22868-74.

Chen Y, Struhl G. (1996). Dual roles for Patched in sequestering and transducing Hedgehog. Cell 87, 553-63.

Choi K-W, Benzer S. (1994). Rotation of photoreceptor clusters in the developing Drosophila eye requires the nemo gene. Cell 78, 125-36. 
Clark LI, Sanghera JS, Pelech SL. (1991). Definition of a consensus sequence for peptide substrate recognition by $\mathrm{p} 44 \mathrm{mpk}$, the meiosis-activated myelin basic protein kinase. J. Biol. Chem. 266, 15180-4.

Cooper MTD, Bray SJ. (2000). R7 photoreceptor specification requires Notch activity. Curr. Biol. 10, 1507-10.

Cornell M, Evans DA, Mann R, Fostier M, Flasza M, Monthatong M, Artavanis-Tsakonas S, Baron M. (1999). The Drosophila melanogaster Suppressor of deltex gene, a regulator of the Notch receptor signaling pathway, is an E3 class ubiquitin ligase. Genetics 152, 567-76.

Cui Y, Jean F, Thomas G, Christian JL. (1998). BMP-4 is proteolytically activated by furin and/or PC6 during vertebrate embryonic development. EMBO J. 17, 4735-43.

Culi J, Modolell J. (1998). Proneural gene self-stimulation in neural precursors: an essential mechanism for sense organ development that is regulated by Notch signaling. Genes Dev. 12, 203647.

Czerny T, Halder G, Kloter U, Souabni A, Gehring WJ, Busslinger M. (1999). twin of eyeless, a second Pax- 6 gene of Drosophila, acts upstream of eyeless in the control of eye development. Mol. Cell 3, 297-307.

de Celis JF, Barrio R, del Arco A, Garcia-Bellido A. (1993). Genetic and molecular characterization of a Notch mutation in its Delta- and Serrate-binding domain in Drosophila. Proc. Natl. Acad. Sci. 90, 4037-41.

de Celis JF, Garcia-Bellido A. (1994). Modifications of the Notch function by Abruptex mutations in Drosophila melanogaster. Genetics 136, 183-94.

de Celis JF, de Celis J, Ligoxygakis P, Preiss A, Delidakis C, Bray S. (1996). Functional relationships between Notch, $S u(H)$ and the bHLH genes of the $E(s p l)$ complex: the $E(s p l)$ genes mediate only a subset of Notch activities during imaginal development. Development 122, 2719-28.

de Celis JF, Bray SJ. (2000). The Abruptex domain of Notch regulates negative interactions between Notch, its ligands and Fringe. Development 127, 1291-302.

del Alamo D, Mlodzik M. (2008). Self-modulation of Notch signaling during ommatidial development via the Roughened eye transcriptional repressor. Development 135, 2895-904.

Delidakis C, Artavanis-Tsakonas S. (1992). The Enhancer of split [E(spl)] locus of Drosophila encodes seven independent helix-loop-helix proteins. Proc. Natl. Acad. Sci. 89, 8731-5.

de Nooij JC, Hariharan IK. (1995). Uncoupling cell fate determination from patterned cell division in the Drosophila eye. Science 270, 983-85.

De Strooper B, Annaert W, Cupers P, Saftif P, Craessaerts K, Mumm JS, Schroeter EH, Schrijvers V, Wolfe MS, Ray WJ, Goate A, Kopan R. (1999). A presinilin-1-dependent gammasecretase-like protease mediates release of Notch intracellular domain. Nature 398, 518-22. 
Distefano GM, Gangemi AJ, Khandelwal PJ, Saunders AJ, Marenda DR. (2012). Drosophila Lilliputian is required for proneural gene expression in retinal development. Dev. Dyn. 241, 553-62.

Dokucu ME, Zipursky SL, Cagan RL. (1996). Atonal, Rough and the resolution of proneural clusters in the developing Drosophila retina. Development 122, 4139-47.

Dominguez M, Hafen E. (1997). Hedgehog directly controls initiation and propagation of retinal differentiation in the Drosophila eye. Genes Dev. 11, 3254-64.

Dominguez M, Wasserman JD, Freeman M. (1998). Multiple functions of the EGF receptor in Drosophila eye development. Curr. Biol. 8, 1039-48.

Dominguez M. (1999). Dual role for Hedgehog in the regulation of the proneural gene atonal during ommatidia development. Development 126, 2345-2353.

Doroquez DB, Rebay I. (2006). Signal integration during development: mechanisms of EGFR and Notch pathway function and cross-talk. Crit. Rev. Biochem. Mol. Biol. 41, 339-85.

Duncan EJ, Dearden PK. (2010). Evolution of a genomic regulatory domain: the role of gene cooption and gene duplication in the Enhancer of split complex. Genome Res. 20, 917-28.

Ellis HM, Spann DR, Posakony JW. (1990). extramacrochaetae, a negative regulator of sensory organ development in Drosophila, defines a new class of helix-loop-helix proteins. Cell 61, 27-38.

Ellis CE, Weber U, Wiersdorff V, Mlodzik M. (1994). Confrontation of scabrous expressing and non-expressing cells is essential for normal ommatidial spacing in the Drosophila eye. Development 120, 1959-69.

Ellis HM. (1994). Embryonic expression and function of the Drosophila helix-loop-helix gene, extramacrochaetae. Mech. Dev. 47, 65-72.

Firth LC, Baker NE. (2009). Retinal determination genes as targets and possible effectors of extracellular signals. Dev. Biol. 327, 366-75.

Firth LC, Bhattacharya A, Baker NE. (2010). Cell cycle arrest by a gradient of Dpp signaling during Drosophila eye development. BMC Dev. Biol. 10, 28.

Fisher AL, Ohsako S, Caudy M. (1996). The WRPW motif of the hairy-related basic helix-loop-helix repressor proteins acts as a 4-amino-acid transcription repression and protein-protein interaction domain. Mol. Cell. Biol. 16, 2670-7.

Fisher AL, Caudy M. (1998). Groucho proteins: transcriptional corepressors for specific subsets of DNA-binding transcription factors in vertebrates and invertebrates. Genes Dev. 12, 1931-40.

Fiuza UM, Klein T, Martinez Arias A, Hayward P. (2010). Mechanisms of ligand-mediated inhibition in Notch signaling activity in Drosophila. Dev. Dyn. 239, 798-805.

Fleming RJ, Gu Y, Hukriede NA. (1997). Serrate-mediated activation of Notch is specifically blocked by the product of the gene fringe in the dorsal compartment of the Drosophila wing imaginal disc. Development 124, 2973-81. 
Frankfort BJ, Nolo R, Zhang Z, Bellen H, Mardon G. (2001). senseless Repression of rough Is Required for R8 Photoreceptor Differentiation in the Developing Drosophila Eye. Neuron 32, 40314.

Frankfort BJ, Mardon G. (2002). R8 development in the Drosophila eye: a paradigm for neural selection and differentiation. Development 129, 1295-306.

Frankfort BJ, Mardon G. (2004). Senseless represses nuclear transduction of Egfr pathway activation. Development 131, 563-70.

Freeman M. (1994). The spitz gene is required for photoreceptor determination in the Drosophila eye where it interacts with the EGF receptor. Mech. Dev. 48, 25-33.

Fu W, Baker NE. (2003). Deciphering synergistic and redundant roles of Hedgehog, Decapentaplegic and Delta that drive the wave of differentiation in Drosophila eye development. Development 130, 5229-39.

Giagtzoglou N, Alifragis P, Koumbanakis KA, Delidakis C. (2003). Two modes of recruitment of E9spl) repressors onto target genes. Development 130, 259-70.

Giagtzoglou N, Koumbanakis KA, Fullard J, Zarifi I, Delidakis C. (2005). Role of the Sc C terminus in transcriptional activation and $\mathrm{E}(\mathrm{spl})$ repressor recruitment. J. Biol. Chem. 280, 1299305

Giebel B, Campos-Ortega JA. (1997). Functional dissection of the Drosophila Enhancer of split protein, a suppressor of neurogenesis. Proc. Natl. Acad. Sci. 94, 6250-4.

Ghattas IR, Sanes JR, Majors JE. (1991). The encephalomyocarditis virus internal ribosome entry site allows efficient coexpression of two genes from a recombinant provirus in cultured cells and in embryos. Mol. Cell. Biol. 11, 5848-59.

Ghazi A, VijayRaghavan K. (2000). Control by combinatorial codes. Dev. Biol. 408, 419-420.

Gonzalez FA, Raden DL, Davis RJ. (1991). Identification of substrate recognition detemrinants for human ERK1 and ERK2 protein kinases. J. Biol. Chem. 266, 22159-63.

Gordon WR, Vardar-Ulu D, Histen G, Sanchez-Irizarry C, Aster JC, Blacklow SC. (2007). Structural basis for autoinhibition of Notch. Nat. Struct. Mol. Biol. 14, 295-300.

Greenwald I, Seydoux G. (1990). Analysis of gain-of-function mutations of the lin-12 gene of Caenorhabditis elegans. Nature 346, 197-9.

Greenwood S, Struhl G. (1999). Progression of the morphogenetic furrow in the Drosophila eye: the roles of Hedgehog, Decapentaplegic and the Raf pathway. Development 126, 5795-808.

Halder G, Callaerts P, Flister S, Walldorf U, Kloter U, Gehring WJ. (1998). Eyeless initiates the expression of both sine oculis and eyes absent during Drosophila compound eye development. Development 125, 2181-91. 
Haynie JL, Bryant PJ. (1986). Development of the eye-antenna imaginal disc and morphogenesis of the adult head in Drosophila melanogaster. J. Exp. Zool. 237, 293-308.

Heberlein U, Wolff T, Rubin GM. (1993). The TGF-beta Homolog $d p p$ and the Segment Polarity Gene hedgehog Are Required for Propagation of a Morphogenetic Wave in the Drosophila retina. Cell 75, 913-26.

Heberlein U, Singh CM, Luk AY, Donohoe TJ. (1995). Growth and differentiation in the Drosophila eye coordinated by hedgehog. Nature 373, 709-11.

Heitzler P, Bourois M, Ruel L, Carteret C, Simpson P. (1996). Genes of the Enhancer of split and achaete-scute complexes are required for a regulatory loop between Notch and Delta during lateral signalling in Drosophila. Development 122, 161-71.

Hicks C, Johnston SH, diSibio G, Collazo A, Vogt TF, Weinmaster G. (2000). Fringe differentially modulates Jagged1 and Delta1 signalling through Notch1 and Notch2. Nat. Cell Biol. 2, 515-20.

Higashijima S, Kojima T, Michiue T, Ishimaru S, Emori Y, Saigo K. (1992). Dual Bar homeo box genes of Drosophila required in two photoreceptor cells, R1 and R6, and primary pigment cells for normal eye development. Genes Dev. 6, 50-60.

Housden BE, Fu AQ, Krejci A, Bernard F, Fischer B, Tavare S, Russell S, Bray SJ. (2013). Transcriptional Dynamics Elicited by a Short Pulse of Notch Activation Involves Feed-Forward Regulation by E(spl)/Hes Genes. PLOS Genet. 9, e1003162.

Inlay MA, Bhattacharya D, Sahoo D, Serwold T, Seita J, Karsunky J, Plevritis SK, Dill DL, Weissman IL. (2009). Ly6d marks the earliest stage of B-cell specification and identifies the branchpoint between B-cell and T-cell development. Genes Dev. 23, 2376-81.

Iritani BM, Forbush KA, Farrar MA, Perlmutter RM. (1997). Control of B cell development by Ras-mediated activation of Raf. EMBO J. 16, 7019-31.

Irvine KD, Wieschaus E. (1994). Fringe, a boundary-specific signaling molecule, mediates interactions between dorsal and ventral cells during Drosophila wing development. Cell 79, 595606.

Irving J, Matheson E, Minto L, Blair H, Case M, Halsey C, Swidenbank I, Ponthan F, KirshcnerSchwabe R, Groeneveld-Krentz S, Hof J, Allan J, Harrison C, Vormoor J, von Stackelberg A, Eckert C. (2014). Ras pathway mutations are prevalent in relapsed childhood acute lymphoblastic leukemia and confer sensitivity to MEK inhibition. Blood 124, 890.

Ishitani T, Kishida S, Hyodo-Miura J, Ueno N, Yasuda J, Waterman M, Shibuya H, Moon RT, Ninomiya-Tsuji J, Matsumoto K. (2003). The TAK1-NLK mitogen-activated protein kinase cascade functions in the Wnt-5a/Ca2+ pathway to antagonize Wnt/beta-catenin signaling. Mol. Cell. Biol. 23, $131-9$.

Jarman AP, Grell EH, Ackerman L, Jan LY, Jan YN. (1994). atonal is the proneural gene for Drosophila photoreceptors. Nature 369, 398-400. 
Jarman AP, Sun Y, Jan LY, Jan YN. (1995). Role of the proneural gene, atonal, in formation of Drosophila chordotonal organs and photoreceptors. Development 121, 2019-30.

Jazwinska A, Kirov N, Wieschaus E, Roth S, Rushlow C. (1999). The Drosophila gene brinker reveals a novel mechanism of Dpp target gene regulation. Cell 96, 563-73.

Jennings B, Preiss A, Delidakis C, Bray S. (1994). The Notch signalling pathway is required for Enhancer of split bHLH protein expression during neurogenesis in the Drosophila embryo.

Development 120, 3537-48.

Jennings BH, Tyler DM, Bray SJ. (1999). Target Specificities of Drosophila Enhancer of split Basic Helix-Loop-Helix Proteins. Mol. Cell. Biol. 19, 4600-10.

Jennings BH, Pickles LM, Wainwright SM, Roe SM, Pearl LH, Ish-Horowicz D. (2006). Molecular recognition of transcriptional repressor motifs by the WD domain of the Groucho/TLE corepressor. Mol. Cell 22, 645-55.

Jia J, Amanai K, Wang G, Tang J, Wang B, Jiang J. (2002). Shaggy/GSK3 antagonizes Hedgehog signalling by regulating Cubitus interruptus. Nature 416, 548-52.

Jia J, Tong C, Jiang J. (2003). Smoothened transduces Hedgehog signal by physically interacting with Costal2/Fused complex through its C-terminal tail. Genes Dev. 17, 2709-20.

Jiang J, Struhl G. (1996). Complementary and mutually exclusive activities of decapentaplegic and wingless organize axial patterning during Drosophila leg development. Cell 86, 401-9.

Kahali B, Bose A, Karandikar U, Bishop CP, Bidwai AP. (2009). On the Mechanism Underlying the Divergent Retinal and Bristle Defects of M8* $\left(E(s p l)^{D}\right)$ in Drosophila. Genesis 00, 1-13.

Kahali B, Kim J-E, Karandikar U, Bishop CP, Bidwai AP. (2010). Evidence that the C-Terminal Domain (CtD) Autoinhibits Neural Repression by Drosophila E(spl)M8. Genesis 48, 44-55.

Karandikar UC, Trott RL, Yin J, Bishop CP, Bidwai AP. (2004). Drosophila CK2 regulates eye morphogenesis via phosphorylation of E(spl)M8. Mech. of Dev. 121, 273-86.

Kelley MR, Kidd S, Deutch WA, Young MW. (1987). Mutations altering the structure of epidermal growth factor-like coding sequences at the Drosophila Notch locus. Cell 51, 539-48.

Kidd S, Kelley MR, Young MW. (1986). Sequence of the notch locus of Drosophila melanogaster: relationship of the encoded protein to mammalian clotting and growth factors. Mol. Cell. Biol. 6, 3094-108.

Kindler T, Cornejo MG, Scholl C, Liu J, Leeman DS, Haydu JE, Frohling S, Lee BH, Gilliland DG. (2008). K-RasG12D-induced T-cell lymphoblastic lymphoma/leukuemias harbor Notch1 mutations and are sensitive to gamma-secretase inhibitors. Blood 112, 3373-82.

Kimmel BE, Heberlein U, Rubin GM. (1990). The homeo domain protein rough is expressed in a subset of cells in the developing Drosophila eye where it can specify photoreceptor cell subtype. Genes Dev. 4, 712-27. 
Klambt C, Knust E, Tietze K, Campos-Ortega JA. (1989). Closely related transcripts encoded by the neurogenic gene complex Enhancer of split of Drosophila melanogaster. EMBO J. 8, 203-10.

Knust E, Schrons H, Grawe F, Campos-Ortega JA. (1992). Seven Genes of the Enhancer of split Complex of Drosophila melanogaster Encode Helix-Loop-Helix Proteins. Genetics 132, 505-18.

Kohler RE. (1994). Lords of the Fly: Drosophila Genetics and Experimental Life. University of Chicago Press. Chicago, IL.

Kojima T, Ishimaru S, Higashijima S-I, Takayama E, Akimaru H, Sone M, Emori Y, Saigo K. (1991). Identification of a different-type homeobox gene, BarH1, possibly causing Bar $(B)$ and Om(1D) mutations in Drosophila. Proc. Natl. Acad. Sci. 88, 4343-7.

Kretzchmar M, Liu F, Hata A, Doody J, Massague J. (1997). The TGF-beta family mediator Smad1 is phosphorylated directly and activated functionally by the BMP receptor kinase. Genes Dev. 11, 984-95.

Kronhamn J, Frei E, Daube M, Jiao R, Shi Y, Noll M, Rasmuson-Lestander A. (2002). Headless flies produced by mutations in the paralogous Pax6 genes eyeless and twin of eyeless. Development 129, 1015-26.

Kumar JP, Moses K. (2001a). EGF Receptor and Notch signaling act upstream of Eyeless/Pax6 to control eye specification. Cell 104, 687-97.

Kumar JP, Moses K. (2001b). The EGF receptor and notch signaling pathways control the initiation of the morphogenetic furrow during Drosophila eye development. Development 128, 2689-2697.

Kumar JP. (2009) The molecular circuitry governing retinal determination. Bio. Biophys. Acta 1789, 306-14.

Kumar JP. (2012). Building an Ommatidium One Cell at a Time. Dev. Dyn. 241, 136-49.

Kunnapuu J, Bjorkgren I, Shimmi 0. (2009). The Drosophila DPP signal is produced by cleavage of its proprotein at evolutionary diversified furin-recognition sites. Proc. Natl. Acad. Sci. 106, 8501-6.

Lai EC, Rubin GM. (2001). neuralized is essential for a subset of Notch pathway-dependent cell fate decision during Drosophila eye development. Proc. Natl. Acad. Sci. 98, 5637-42.

Lai Z-C, Rubin GM. (1992). Negative Control of Photoreceptor Development in Drosophila by the Product of the yan Gene, an ETS Domain Protein. Cell 70, 609-20.

Lake RJ, Grimm LM, Veraksa A, Banos A, Artavanis-Tsakonas S. (2009). In Vivo Analysis of the Notch Receptor S1 Cleavage. PLOS One 4, e6728.

LeBon L, Lee TV, Sprinzak D, Jafar-Nejad H, Elowitz MB. (2014). Fringe proteins modulate Notch-ligand cis and trans interactions to specify signaling states. eLife 3, e02950.

Lecourtois M, Schweisguth F. (1995). The neurogenic suppressor of hairless DNA-binding protein mediates the transcriptional activation of the enhancer of split complex genes triggered by Notch signaling. Genes Dev. 9, 2598-608. 
Lee JJ, Ekker SC, von Kessler DP, Porter JA, Sun BI, Beachy PA. (1994). Autoproteolysis in hedgehog protein biogenesis. Science 266, 1528-37.

Lee JR, Urban S, Garvey CF, Freeman M. (2001). Regulated intracellular ligand transport and proteolysis control EGF signal activation in Drosophila. Cell 107, 161-71.

Lei L, Xu A, Panin VM, Irvine KD. (2003). An O-fucose site in the ligand binding domain inhibits Notch activation. Development 130, 6411-21.

Lemmon MA, Bu Z, Ladbury JE, Zhou M, Pinchasi D, Lax I, Engelman DM, Schlessinger J. (1997). Two EGF molecules contribute additively to stabilization of the EGFR dimer. EMBO J. 16, 281-94.

Lesokhin AM, Yu S-Y, Katz J, Baker NE. (1999). Several Levels of EGF Receptor Signaling during Photoreceptor Specificaiton in Wild-Type, Ellipse, and Null Mutant Drosophila. Dev. Biol. 205, 12944.

Lewis EB. (1992). Clusters of master control genes regulate the development of higher organisms. J. Am. Medical Assoc. 267, 1524-31.

Li Y, Baker NE. (2001). Proneural enhancement by Notch overcomes Suppressor-of-Hairless repressor function in the developing Drosophila eye. Curr. Biol. 11, 330-8.

Li Y, Li L, Irvine KD, Baker NE. (2003). Notch acitivity in neural cells triggered by a mutant allele with altered glycosylation. Development 130, 2829-40.

Li Y, Jiang Y, Chen Y, Karandikar U, Hoffman K, Chattopadhyay A, Mardon G, Chen R. (2013). optix functions as a link between the retinal determination network and the $d p p$ pathway to control morphogenetic furrow progression in Drosophila. Dev. Biol. 381, 50-61.

Lieber T, Kidd S, Alcamo E, Corbin V, Young MW. (1993). Antineurogenic phenotypes induced by truncated Notch proteins indicate a role in signal transduction and may point to a novel function for Notch in nuclei. Genes Dev. 7, 1949-65.

Ligoxygakis P, Yu S-Y, Delidakis C, Baker NE. (1998). A subset of Notch functions during Drosophila eye development require $S u(H)$ and the $E(s p l)$ gene complex. Development 125, 2893900.

Lim J, Choi K-W. (2003). Bar homeodomain proteins are anti-proneural in the Drosophila eye: transcriptional repression of atonal by Bar prevents ectopic retinal neurogenesis. Development 130, 5965-74.

Lim J, Choi K-W. (2004). Induction and autoregulation of the anti-proneural gene Bar during retinal neurogenesis in Drosophila. Development 131, 5573-80.

Lim J, Jafar-Nejad H, Hsu Y-C, Choi K-W. (2008). Novel function of the class I bHLH protein Daughterless in the negative reguilation of proneural gene expression in the Drosophila eye. EMBO Reports 9, 1128-33. 
Lindsley DL, Zimm GG. (1992). The Genome of Drosophila melanogaster. New York: Academic Press.

Liu P, Cleveland IV TE, Bouyain S, Byrne PO, Longo PA, Leahy DJ. (2012). A single ligand is sufficient to activate EGFR dimers. Proc. Natl. Acad. Sci. 109, 10861-6.

Lubensky DK, Pennington MW, Shraiman BI, Baker NE. (2011). A dynamical model of ommatidial crystal formation. Proc. Natl. Acad. Sci. 108, 11145-50.

Lum L, Zhang C, Oh S, Mann RK, von Kessler DP, Taipale J, Weis-Garcia F, Gong R, Wang B, Beachy PA. (2003). Hedgehog Signal Transduction via Smoothened Association with a Cytoplasmic Complex Scaffolded by the Atypical Kinesin, Costal-2. Mol. Cell 12, 1261-74.

Ma C, Zhou Y, Beachy PA, Moses K. (1993). The Segment Polarity Gene hedgehog Is Required for Progression of the Morphogenetic Furrow in the Developing Drosophila Eye. Cell 75, 927-38.

Macias-Silva M, Abdollah S, Hoodless PA, Pirone R, Attisano L, Wrana JL. (1996). MADR2 Is a Substrate of the TGF-beta Receptor and Its Phosphorylation Is Required for Nuclear Accumulation and Signaling. Cell 87, 1215-24.

Maeder ML, Polansky BJ, Robson BE, Eastman DA. (2007). Phylogenetic Footprinting Analysis in the Upstream Regulatory Regions of the Drosophila Enhancer of split Genes. Genetics 177, 1377-94.

Majot AM, Sizemore TR, Bandyopadhyay M, Jozwick LM, Bidwai AP. (2015). Protein Kinase CK2: A Window into the Posttranslational Regulation of the E(spl)/HES Repressors from Invertebrates and Vertebrates. In Ahmed K, Issinger O-G, Szyszka R (Eds.), Protein Kinase CK2 Cellular Function in Normal and Disease States (81-107). New York: Springer.

Mardon G, Solomon NM, Rubin GM. (1994). dachshund encodes a nuclear protein required for normal eye and leg development in Drosophila. Development 120, 3473-86.

Martin AC, Goldstein B. (2014). Apical constriction: themes and variations on a cellular mechanism driving morphogenesis. Development 141, 1987-98.

Marty T, Muller B, Basler K, Affolter M. (2000). Schnurri mediates Dpp-dependent repression of brinker transcription. Nat. Cell Biol. 2, 745-9.

Matsuno K, Diederich RJ, Go MJ, Blaumueller CM, Artavanis-Tsakonas S. (1995). Deltex acts as a positive regulator of Notch signaling through interactions with the Notch ankyrin repeats.

Development 121, 2633-44.

Melicharek D, Shah A, DiStefano G, Gangemi AJ, Orapallo A, Vrailas-Mortimer AD, Marenda DR. (2008). Identification of Novel Regulators of atonal Expression in the Developing Drosophila Retina. Genetics 180, 2095-110.

Methot N, Basler K. (1999). Hedgehog Controls Limb Development by Regulating the Activities of Distinct Transcriptional Activator and Repressor Forms of Cubitus interruptus. Cell 96, 819-31.

Minami M, Kinoshita N, Kamoshida Y, Tanimoto H, Tabata T. (1999). brinker is a target of Dpp in Drosophila that negatively regulates Dpp-dependent genes. Nature 398, 242-6. 
Moloney DJ, Panin VM, Johnston SH, Chen J, Shao L, Wilson R, Wang Y, Stanley P, Irvine KD, Haltiwanger RS, Vogt TF. (2000). Fringe is a glycosyltransferase that modifies Notch. Nature 406, 369-75.

Morel V, Schweisguth F. (2000) Repression by Suppressor of Hairless and activation by Notch are required to define a single row of single-minded expressing cells in the Drosophila embryo. Genes Dev. 14, 377-88.

Morel V, Lecourtois M, Massiani O, Maier D, Preiss A, Schweisguth F. (2001). Transcriptional repression by Suppressor of Hairless involves the binding of a Hairless-dCtBP complex in Drosophila. Curr. Biol. 11, 789-92.

Morillo SA, Braid LR, Verheyn EM, Rebay I. (2012). Nemo phosphorylates Eyes absent and enhances output from the Eya-Sine oculis transcriptional complex during Drosophila retinal determination. Dev. Biol. 365, 267-76.

Motzny CK, Holmgren R. (1995). The Drosophila cubitus interruptus protein and its role in the wingless and hedgehog signal transduction pathways. Mech. Dev. 52, 137-50.

Nagel AC, Preiss A. (1999). Notch ${ }^{s p l}$ Is Deficient for Inductive Processes in the Eye, and E(spl)D Enhances split by Interfering with Proneural Activity. Dev. Biol. 208, 406-15.

Nagel AC, Yu Y, Preiss A. (1999). Enhancer of Split [E(spl)D] is a Gro-independent, hypermorphic mutation in Drosophila. Dev. Gen. 25, 168-79.

Nakao K, Campos-Ortega JA. (1996). Persistent expression of genes of the Enhancer of Split complex suppresses neural development in Drosophila. Neuron 16, 275-86.

Nellesen DT, Lai EC, Posakony JW. (1999). Discrete Enhancer Elements Mediate Selective Responsiveness of Enhancer of split Complex Genes to Common Transcriptional Activators. Dev. Biol. 213, 33-53.

Nolo R, Abbott LA, Bellen HJ. (2000). Senseless, a Zn Finger Transcription Factor, Is Necessary and Sufficient for Sensory Organ Development in Drosophila. Cell 102, 349-62.

Oberg C, Li J, Pauley A, Wolf E, Gurney M, Lendahl U. (2001). The Notch intracellular domain is ubiquitinated and negatively regulated by the mammalian Sel-10 homolog. J. Biol. Chem. 276, 35847-53.

Oellers N, Tietze K, Knust E. (1993). The genes of the Enhancer of split complex of Drosophila melanogaster encodes regulatory helix-loop-helix proteins. J. Neurogenetics $\mathbf{8}, 242$.

Ohsako S, Hyer J, Panganiban G, Oliver I, Caudy M. (1994). Hairy function as a DNA-binding helix-loop-helix repressor of Drosophila sensory organ formation. Genes. Dev. 8, 2743-55.

Okajima T, Irvine KD. (2002). Regulation of Notch signaling by 0-linked fucose. Cell 111, 893-904.

Okajima T, Xu A, Lei L, Irvine KD. (2005). Chaperone activity of protein O-fucosyltransferase 1 promotes Notch receptor folding. Science 307, 1599-603. 
Okochi M, Steiner H, Fukumori A, Tanii H, Tomita T, Tanaka T, Iwatsubo T, Kudo T, Takeda M, Haass C. (2002). Presenilins mediate a dual intramembranous gamma-secretase cleavage of Notch-1. EMBO J. 21, 5408-16.

Olson ER, Pancratov R, Chatterjee SS, Changkakoty B, Pervaiz Z, DasGupta R. (2011). Yan, an ETS-domain transcription factor, negatively modulates the Wingless pathway in the Drosophila eye. EMBO Reports 12, 1047-54.

O'Neill EM, Rebay I, Tjian R, Rubin GM. (1994). The Activities of Two Ets-Related Transcription Factores Required for Drosophila Eye Development Are Modulated by the Ras/MAPK Pathway. Cell 78, 137-47.

Panin VM, Papayannopoulos , Wilson R, Irvine KD. (1997). Fringe modulates Notch-ligand interactions. Nature 387, 908-12.

Panin VM, Shao L, Lei L, Moloney DJ, Irvine KD, Haltiwanger RS. (2002). Notch ligands are substrates for protein 0-fucosyltransferase-1 and Fringe. J. Biol. Chem. 277, 29945-52.

Pappu KS, Chen R, Middlebrooks BW, Woo C, Heberlein U, Mardon G. (2003). Mechanism of hedgehog signaling during Drosophila eye development. Development 130, 3053-62.

Pappu KS, Ostrin EJ, Middlebrooks BW, Sili BT, Chen R, Atkins MR, Gibbs R, Mardon G. (2005) Dual regulation and redundant function of two eye-specific enhancers of the Drosophila retinal determination gene dachshund. Development 132, 2895-905.

Parks AL, Stout JR, Shepard SB, Klueg KM, Dos S, Parody TR, Vaskova M, Muskavitch MAT. (2006). Structure-function analysis of Delta trafficking, receptor binding and signaling in Drosophila. Genetics 174, 1947-61.

Paroush, Z, Finley RL Jr., Kidd T, Wainwright SM, Ingham PW, Brent R, Ish-Horowicz D. (1994). Groucho is required for Drosophila neurogenesis, segmentation, and sex determination and interacts directly with hairy-related bHLH proteins. Cell 79, 805-15.

Pauli T, Seimiya M, Blanco J, Gehring WJ. (2005). Identification of functional sine oculis motifs in the autoregulatory element of its own gene, in the eyeless enhancer and in the signalling gene hedgehog. Development 132, 2771-82.

Pei Z, Baker NE. (2008). Competition between Delta and the Abruptex domain of Notch. BMC Dev. Biol. 8, 1-14.

Pepple KL, Anderson AE, Frankfort BJ, Mardon G. (2007). A Genetic Screen in Drosophila for Genes Interacting With senseless During Neuronal Development Identifies the Importin moleskin. Genetics 175, 125-41.

Pepple KL, Atkins M, Venken K, Wellnitz K, Harding M, Frankfort B, Mardon G. (2008). Twostep selection of a single R8 photoreceptor: a bistable loop between senseless and rough locks in R8 fate. Development 135, 4071-79. 
Petcherski AH, Kimble J. (2000). LAG-3 is a putative transcriptional activator in the C. elegans Notch pathway. Nature 405, 364-8.

Poortinga G, Watanabe M, Parkhurst SM. (1998). Drosophila CtBP: a hairy-interacting protein required for embryonic segmentation and hairy-mediated transcriptional repression. EMBO J. 17, 2067-78.

Potier D, Davie K, Hulselmans G, Sanchez MN, Haagen L, Huynh-Thu VA, Koldere D, Celik A, Geurts P, Christiaens V, Aerts S. (2014). Mapping Gene Regulatory Networks in Drosophila Eye Development by Large-Scale Transcriptome Perturbations and Motif Inference. Cell Rep. 9, 2290303.

Porter JA, von Kessler DP, Ekker SC, Young KE, Lee JJ, Moses K, Beachy PA. (1995). The product of hedgehog autoproteolytic cleavage active in local and long-range signaling. Nature 374, 363-6.

Porter JA, Ekker SC, Park WJ, von Kessler DP, Young KE, Chen CH, Ma Y, Woods AS, Cotter RJ, Koonin EV. (1996). Hedgehog patterning activity: role of a lipophilic modification mediated by the carboxy-terminal autoprocessing domain. Cell 86, 21-34.

Powell LM, zur Lage PI, Prentice DRA, Senthinathan B, Jarman AP. (2004). The proneural proteins Atonal and Scute regulate neural target genes through different E-box binding sites. Mol. Cell. Biol. 24, 9517-26.

Price MA, Calderon D. (2002). Proteolysis of the Hedgehog Signaling Effector Cubitus interruptus Requires Phosphorylation by Glycogen Synthase Kinase 3 and Casein Kinase 1. Cell 108, 823-35.

Pui JC, Allman D, Xu L, DeRocco S, Karnell FG, Bakkour S, Lee JY, Kadesch T, Hardy RR, Aster JC, Pear WS. (2009). Notch1 expression in early lymphopoiesis influences B cersus T lineage determination. Immunity 11, 299-308.

Radtke F, Wilson A, Mancini SJ, MacDonal HR. (1999) Notch regulation of lymphocyte development and function. Immunity 10, 547-58.

Raftery LA, Twombly V, Wharton KA, Gelbart WM. (1995). Genetic screens to identify elements of the decapentaplegic signaling pathway in Drosophila. Genetics 139, 241-54.

Raimondi SC, Shurtleff SA, Downing JR, Rubnitz J, Mathew S, Hancock M, Pui C-H, Rivera GK, Grosveld GC, Behm FG. (1997) 12p abnormalities and the TEL gene (ETV6) in childhood acute lymphoblastic leukemia. Blood 90 4559-66.

Ramain P, Khechumian K, Seugnet L, Arbogast N, Ackermann C, Heitzler P. (2001). Novel Notch alleles reveal a Deltex-dependent pathway repressing neural fate. Curr. Biol. 11, 1729-38.

Ramos E. (2003). Unraveling the Functional Motifs of the yan Enhancer in Drosophila melanogaster. Pennsylvania State University Graduate School Dissertation.

Ramos E, Price M, Rohrbaugh M, Lai Z-C. (2003). Identifying functional cis-acting regulatory modules of the yan gene in Drosophila melanogaster. Dev. Genes Evol. 213, 83-9. 
Rebay I, Fleming RJ, Fehon RG, Cherbas L, Cherbas P, Artavanis-Tsakonas S. (1991). Specific EGF repeats of Notch mediate interactions with Delta and Serrate: Implications for Notch as a mulfunctional receptor. Cell 67, 687-99.

Ready DF, Hanson TE, Benzer S. (1976). Development of the Drosophila retina, a neurocrystalline lattice. Dev. Biol. 53, 217-40.

Rebay I, Rubin GM. (1995). Yan Functions as a General Inhibitor of Differentiation and Is Negatively Regulated by Activation of the Ras1/MAPK Pathway. Cell 81, 857-66.

Riggleman B, Schedl P, Wieschaus E. (1990). Spatial expression of the Drosophila segment polarity gene armadillo is posttranscriptionally regulated by wingless. Cell 63, 549-60.

Robbins DJ, Nybakken KE, Kobayashi R, Sisson JC, Bishop JM, Therond PP. (1997). Hedgehog Elicits Signal Transduction by Means of a Large Complex Containing the Kinesin-Related Protein Costal2. Cell 90, 225-34.

Rodrigues AB, Werner E, Moses K. (2005). Genetic and biochemical analysis of the role of Egfr in the morphogenetic furrow of the developing Drosophila eye. Development 132, 4697-707.

Roignant J-Y, Treisman JE. (2009). Pattern formation in the Drosophila eye disc. Int. J. Dev. Biol. 53, 795-804.

Rogers EM, Brennan CA, Mortimer NT, Cook S, Morris AR, Moses K. (2005) Pointed regulates an eye-specific transcriptional enhancer in the Drosophila hedgehog gene, which is required for the movement of the morphogenetic furrow. Development 132, 4833-43.

Rogge R, Green PJ, Urano J, Horn-Saban S, Mlodzik M, Shilo B-Z, Hartenstein V, Banerjee U. (1995). The role of yan in mediating the choice between cell division and differentiation. Development 121, 3947-3958.

Rohrbaugh M, Ramos E, Nguyen D, Price M, Wen Y, Lai Z-C. (2002). Notch Activation of yan Expression Is Antagonized by RTK/Pointed Signaling in the Drosophila Eye. Curr. Biol. 12, 576-81.

Rosenberg MI, Parkhurst SM. (2002). Drosophila Sir2 is required for heterochromatic silencing and by euchromatic hairy/E(spl) bHLH repressors in segmentation and sex determination. Cell 109, 447-58.

Roukens MG, Alloul-Ramdhani M, Vertegaal ACO, Anvarian Z, Balog CIA, Deelder AM, Hensbergen PJ, Baker DA. (2008a). Identification of a new site of sumoylation on Tel (ETV6) uncovers a PIAS-dependent mode of regulation Tel function. Mol. Cell. Biol. 28, 2342-57.

Roukens MG, Alloul-Ramdhani M, Moghadasi S, Op den Brouw M, Baker DA. (2008b). Downregulation of vertebrate Tel (ETV6) and Drosophila Yan is facilitated by an evolutionarily conserved mechanism of F-box-mediated ubiquitination. Mol. Cell. Biol. 28, 4394-4406.

Ruberte E, Marty T, Nellen D, Affolter M, Basler K. (1995). An absolute requirement for both the Type II and Type I receptors, punt and thick veins, for Dpp signaling in vivo. Cell 80, 889-97. 
Ruel L, Rodriguez R, Gallet A, Lavenant-Staccini L, Therond PP. (2003). Stability and association of Smoothened, Costal2 and Fused with Cubitus interruptus are regulated by Hedgehog. Nat. Cell Biol. 5, 907-13.

Rutledge BJ, Zhang K, Bier E, Jan YN, Perrimon N. (1992). The Drosophila spitz gene encodes a putative EGF-like growth factor involved in dorsal-ventral axis formation and neurogenesis. Genes. Dev. 6, 1503-17.

Sakamoto K, Ohara O, Takagi M, Takeda S, Katsube K. (2002). Intracellular cell-autonomous association of Notch and its ligands: a novel mechanism of Notch signal modification. Dev. Biol. 241, 313-26.

Sasamura T, Sasaki N, Miyashita F, Nakao S, Ishikawa HO, Ito M, Kitagawa M, Harigaya K, Spana E, Bilder D, Perrimon N, Matsuno K. (2003). neurotic, a novel maternal neurogenic gene, encodes an 0-fucosyltransferase that is essential for Notch-Delta interactions. Development 130, 4785-95.

Sasamura T, Ishikawa HO, Sasaki N, Higashi S, Kanai M, Nakao S, Ayukawa T, Aigaki T, Noda K, Miyoshi E, Taniguchi N, Matsuno K. (2007). The O-fucosyltransferase O-fut1 is an extracellular component that is essential for the constitutive endocytic trafficking of Notch in Drosophila.

Development 134, 1347-1356.

Sato M, Kojima T, Michiue T, Saigo K. (1999). Bar homeobox genes are latitudinal prepattern genes in the developing Drosophila notum whose expression is regulated by the concerted functions of deceapentaplegic and wingless. Development 126, 1457-66.

Sawyer JM, Harrell JR, Shemer G, Sullivan-Brown J, Roh-Johnson M, Goldstein B. (2010). Apical Constriction: A Cell Shape Change that Can Drive Morphogenesis. Dev. Biol. 341, 5-19.

Schaaf CA, Misulovin Z, Gause M, Koenig A, Dorsett D. (2013). The Drosophila enhancer of split gene complex: Architecture and coordinate regulation by notch, cohesion, and polycomb group proteins. G3 3, 1785-94.

Schlatter R, Maier D. (2005). The Enhancer of split and Achaete-Scute complexes of Drosophilids derived from simple ur-complexes preserved in mosquito and honeybee. BMC Evo. Biol. 5, 67.

Schlessinger J. (1988). Signal transduction by allosteric receptor oligomerization. Trends Biochem. Sci. 13, 443-7.

Schlessinger J. (2000). Cell signaling by receptor tyrosine kinases. Cell 103, 211-25.

Scholz H, Deatrick J, Klaes A, Klambt C. (1993). Genetic dissection of pointed, a Drosophila gene encoding two ETS-related proteins. Genetics 135, 455-68.

Schrons H, Knust E, Campos-Ortega JA. (1992) The Enhancer of split complex and adjacent genes in the $96 \mathrm{~F}$ region of Drosophila melanogaster are required for segregation of neural and epidermal progenitor cells. Genetics 132, 481-503.

Schuldt AJ, Brand AH. (1999). Mastermind acts downstream of notch to specify neuronal cell fates in the Drosophila central nervous system. Dev. Biol. 205, 287-95. 
Schweitzer R, Shilo B. (1997). A thousand and one roles for the Drosophila EGF receptor. Trends Gen. 13, 191-6.

Sekelsky JJ, Newfeld SJ, Raftery LA, Chartoff EH, Gelbart WG. (1995). Genetic characterization and cloning of Mothers against dpp, a gene required for decapentaplegic function in Drosophila melanogaster. Genetics 139, 1347-58.

Shao L, Moloney DJ, Haltiwanger R. (2003). Fringe modifies 0-fucose on mouse Notch1 at epidermal growth factor-like repeats within the ligand-binding site and the Abruptex region. J. Biol. Chem. 278, 7775, 82.

Shimizu K, Chiba S, Kumano K, Hosoya N, Takahashi T, Kanda Y, Hamada Y, Yazaki Y, Hirai H. (1999). Mouse jagged1 physically interacts with notch2 and other notch receptors. Assessment by quantitative methods. J. Biol. Chem. 274, 32961-9.

Simin K, Bates EA, Horner MA, Letsou A. (1997). Genetic Analysis of Punt, a Type II Dpp Receptor That Functions Throughout the Drosophila melanogaster Life Cycle. Genetics 148, 801-13.

Singer MA, Penton A, Twombly V, Hoffmann FM, Gelbart WM. (1997). Signaling through both type I DPP receptors is required for anterior-posterior patterning of the entire Drosophila wing. Development 124, 79-89.

Singson A, Levitan M, Bang AG, Hua XH, Posakony JW. (1994). Direct downstream targets of proneural activators in the imaginal disc include genes involved in lateral inhibitory signaling. Genes Dev. 8, 2058-71.

Sisson JC, Ho KS, Suyama K, Scott MP. (1997). Costal2, a Novel Kinesin-Related Protein in the Hedgehog Signaling Pathway. Cell 90, 235-45.

Smoller DA, Friedel C, Schmid A, Bettler D, Lam L, Yedvobnick B. (1990). The Drosophila neurogenic locus mastermind encodes a nuclear protein unusually rich in amino acid homopolymers. Genes Dev. 4, 1688-700.

Spratford CM, Kumar JP. (2013). Extramacrochaetae imposes order on the Drosophila eye by refining the activity of the Hedgehog signaling gradient. Development 140, 1994-2004.

St. Pierre SE, Galindo MI, Couso JP, Thor S. (2002). Control of Drosophila imaginal disc development by rotund and roughened eye: differentially expressed transcripts of the same gene encoding functionally distinct zinc finger proteins. Development 129, 1273-81.

Stahl M, Uemura K, Ge C, Shi S, Tashima Y, Stanley P. (2008). Roles of Pofut1 and O-fucose in mammalian Notch signaling. J. Biol. Chem. 283, 13638-51.

Strutt DI, Mlodzik M. (1996). The regulation of hedgehog and decapentaplegic during Drosophila eye imaginal disc development. Mech. Dev. 58, 39-50.

Strutt DI, Mlodzik M. (1997). Hedgehog is an indirect regulator of morphogenetic furrow progression in the Drosophila eye disc. Development 124, 3233-40. 
Struhl G, Greenwald J. (1999). Presenilin is required for activity and nuclear access of Notch in Drosophila. Nature 398, 522-5.

Sun Y, Jan LY, Jan YN. (1998). Transcriptional regulation of atonal during development of the Drosophila peripheral nervous system. Development 125, 3731-40.

Taelman V, Van Wayenbergh R, Solter M, Pichon B, Pieler T, Christophe D, Bellefroid EJ. (2004). Sequences downstream of the bHLH domain of the Xenopus hairy-related transcription factor- 1 act as an extended dimerization domain that contributes to the selection of the partners. Dev. Biol. 276, 47-63.

Taipale J, Cooper MK, Maiti T, Beachy PA. (2002). Patched acts catalytically to suppress the activity of Smoothened. Nature 418, 892-6.

Takeuchi H, Fernandez-Valdivia RC, Caswell DS, Nita-Lazar A, Rana NA, Garner TP, Weldeghiorghis TK, Macnaughtan MA, Jafar-Nejad H, Haltiwanger RS. (2011). Rumi functions as both a protein 0-glucosyltransferase and a protein 0 -xylosyltransferase. Proc. Natl. Acad. Sci. 108, 16600-5.

Tanaka-Matakatsu M, Du W. (2008). Direct control of the proneural gene atonal by retinal determination factors during Drosophila eye development. Dev. Biol. 313, 787-801.

Tanaka-Matakatsu M, Miller J, Borger D, Tang W-J, Du W. (2012). Daughterless homodimer synergizes with Eyeless to induce Atonal expression and retinal neuron differentiation. Dev. Biol. 392, 256-65.

Tang AH, Neufeld TP, Rubin GM, Muller HA. (2001). Transcriptional regulation of cytoskeletal functions and segmentation by a novel maternal pair-rule gene, lilliputian. Development 128, 80113.

Tei H, Nihonmatsu I, Yokokura T, Ueda R, Sano Y, Okuda T, Sato K, Hirata K, Fujita SC, Yamamoto D. (1992). pokkuri, a Drosophila gene encoding an E-26-specific (Ets) domain protein, prevents overproduction of the R7 photoreceptor. Proc. Natl. Acad. Sci. 89, 6856-60.

Terracol R, Lengyel JA. (1994). The thick veins gene of Drosophila is required for dorsoventral polarity of the embryo. Genetics 138, 165-78.

Tien AC, Rajan A, Schulze KL, Ryoo HD, Acar M, Steller H, Bellen HJ. (2008). Ero1L, a thiol oxidase, is required for Notch signaling through cysteine bridge formation of the Lin12-Notch repeats in Drosophila. J. Cell Biol. 182, 1113-25.

Tietze K, Oellers N, Knust E. (1992). Enhancer of splitD, a dominant mutation of Drosophila, and its use in the study of functional domains of a helix-loop-helix protein. Proc. Natl. Acad. Sci. 89, 6152-6.

Tio M, Ma C, Moses K. (1994). spitz, a Drosophila homolog of transforming growth factor-alpha is required in the founding photoreceptor cells of the compound eye facets. Mech. Dev. 48, 13-23.

Tio M, Moses K. (1997). The Drosophila TGF homolog Spitz acts in photoreceptor recruitment in the developing retina. Development 124, 343-51. 
Tomlinson A, Kimmel BE, Rubin GM. (1988). rough, a Drosophila homeobox gene required in photoreceptors R2 and R5 for inductive interactions in the developing eye. Cell 55, 771-84.

Tomlinson A, Mavromatakis YE, Struhl G. (2011). Three Distinct Roles for Notch in Drosophila R7 Photoreceptor Specification. PLOS Biology 9, e1001132.

Torres-Vazquez J, Warrior R, Arora K. (2000). schnurri is required for dpp-dependent patterning of the Drosophila wing. Dev. Biol. 227, 388-402.

Treisman JE, Rubin GM. (1995). wingless inhibits morphogenetic furrow movement in the Drosophila eye disc. Development 121, 3519-27.

Troost T, Schneider M, Klein T. (2015). A Re-examination of the Selection of the Sensory Organ Precursor of the Bristle Sensilla of Drosophila melanogaster. PLOS Genetics 11, e1004911.

Trott RL, Kalive M, Paroush Z, Bidwai AP. (2001). Drosophila melanogaster Casein Kinase II Interacts with and Phosphorylates the Basic Helix-Loop-Helix Proteins m5, m7, and m8 Derived from the Enhancer of split Complex. J. Biol. Chem. 276, 2159-2167.

Tsruya R, Schlesinger A, Reich A, Gabay L, Sapir A, Shilo BZ. (2002). Intracellular trafficking by Star regulates cleavage of the Drosophila EGF receptor ligand Spitz. Genes Dev. 16, 222-34.

Urban S, Lee JR, Freeman M. (2001). Drosophila rhomboid-1 defines a family of putative intramembrane serine proteases. Cell 107, 173-82.

van Doren M, Ellis HM, Posakony JW. (1991). The Drosophila extramacrochaetae protein antagonizes sequence-specific DNA binding by daughterless/achaete-scute protein complexes. Development 113, 245-55.

Venken KJ, Schulze KL, Haelterman NA, Pan H, He Y, Evans-Holm M, Carlson JW, Levis RW, Spradling AC, Hoskins RA, Bellen HJ. (2011). MiMIC: a highly versatile transposon insertion resource for engineering Drosophila melanogaster genes. Nat. Methods 8, 737-43.

Ventura F, Doody J, Liu F, Massague J. (1994). Reconstitution and transphosphorylation of TGFbeta receptor complexes. EMBO J. 13, 5581-9.

Verheyen EM, Purcell KJ, Fortini ME, Artavanis-Tsakonas S. (1996). Analysis of dominant enhances and suppressors of activated Notch in Drosophila. Genetics 144, 1127-41.

Vivekanand P, Rebay I. (2006). Intersection of signal transduction pathways and development. Ann. Rev. Genet. 40, 139-57.

Wainwright SM, Ish-Horowicz D. (1992). Point mutations in the Drosophila hairy gene demonstrate in vivo requirements for basic, helix-loop-helix, and WRPW domains. Mol. Cell. Biol. 12, 2475-83.

Wang H, Chattopadhyay A, Li Z, Daines B, Li Y, Gao C, Gibbs C, Zhang K, Chen R. (2010). Rapid identification of heterozygous mutations in Drosophila melanogaster using genomic capture sequencing. Genome Res. 20, 981-8. 
Wang W, Struhl G. (2004). Drosophila Epsin mediates a select endocytic pathway that DSL ligands must enter to activate Notch. Development 131, 5367-80.

Wang W, Struhl G. (2005). Distinct roles for Mind bomb, Neuralized and Epsin in mediating DSL endocytosis and signaling in Drosophila. Development 132, 2883-94.

Wasserman JD, Urban S, Freeman M. (2000). A family of rhomboid-like genes: Drosophila rhomboid-1 and roughoid/rhomboid-3 cooperate to activate EGF receptor signaling. Genes Dev. 14, 1651-63.

Webber JL, Zhang J, Cote L, Vivekanand P, Ni X, Zhou J, Negre N, Carthew RW, White KP, Rebay I. (2013). The Relationship Between Long-Range Chromatin Occupancy and Polymerization of the Drosophila ETS Family Transcriptional Repressor Yan. Genetics 193, 633-49.

Weis-Garcia F, Massague J. (1996). Complementation between kinase-defective and activationdefective TGF-beta receptors reveals a novel form of receptor cooperativity essential for signaling. EMBO J. 15, 276-89.

Welshons WJ. (1956). Dosage experiments with split mutants in the presence of an enhancer of split. DIS 30, 157-8.

Weng AP, Ferrando AA, Lee W, Morris JP IV, Silverman LB, Sanchez-Izarry C, Blacklow SC, Look AT, Aster JC. (2004). Activating mutations of NOTCH1 in human T cell acute lymphoblastic leukemia. Science 306, 269-71.

Wolff T, Ready DF. (1991). The beginning of pattern formation in the Drosophila compound eye: the morphogenetic furrow and the second mitotic wave. Development 113, 841-50.

Wolff T, Ready DF. (1993). Pattern formation in the Drosophila retina. In: The Development of Drosophila melanogaster. Cold Spring Harbor Laboratory Press. 2, 1277-325.

Wrana JL, Attisano L, Wieser R, Ventura F, Massague J. (1994). Mechanism of activation of the TGF-beta receptor. Nature 370, 341-7.

Xu A, Lei L, Irvine KD. (2005). Regions of Drosophila Notch that contribute to ligand binding and the modulatory influence of Fringe. J. Biol. Chem. 280, 30158-65.

Xu C, Kauffmann RC, Zhang J, Kladny S, Carthew RW. (2000). Overlapping activators and repressors delimit transcriptional response to receptor tyrosine kinase signals in the Drosophila eye. Cell 103, 87-97.

Yang L, Baker NE. (2001). Role of the EGFR/Ras/Raf pathway in specification of photoreceptor cells in the Drosophila retina. Development 128, 1183-91.

Yang L, Baker NE. (2003). Cell Cycle Withdrawal, Progression, and Cell Survival Regulation by EGFR and Its Effectors in the Differentiating Drosophila Eye. Dev. Cell 4, 359-69.

Yeh E, Dermer M, Commisso C, Zhou L, McGlade CJ, Boulianne GL. (2001). Neuralized functions as an E3 ubiquitin ligase during Drosophila development. Curr. Biol. 11, 1675-9. 
Yuh C-H, Bolouri H, Davidson EH. (1998). Genomic Cis-Regulatory Logic: Experimental and Computational Analysis of a Sea Urchin Gene. Science 279, 1896-902.

Zarifi I, Kiparaki M, Koumbanakis KA, Giagtzoglou N, Zacharioudaki E, Alexiadis A, Livadaras I, Delidakis C. (2012). Essential Roles of Da Transactivation Domains in Neurogenesis and in E(spl)-Mediated Repression. Mol. Cell. Biol. 32, 4534-48.

Zhang J, Graham TGW, Vivekanand P, Cote L, Cetera M, Rebay I. (2010). Sterile Alpha Motif Domain-Mediated Self-Association Plays An Essential Role in Modulating the Activity of the Drosophila ETS Family Transcriptional Repressor Yan. Mol. Cell. Biol. 30, 1158-70.

Zhang T, Ranade S, Cai CQ, Clouser C, Pignoni F. (2006). Direct control of neurogenesis by selector factors in the fly eye: regulation of atonal by Ey and So. Development 133, 4881-9.

Zhang T, Zhou Q, Pignoni F. (2011). Yki/YAP, Sd/TEAD and Hth/MEIS Control Tissue Specification in the Drosophila Eye Disc Epithelium. PLOS One 6, e22278.

Zhang X, Gureasko J, Shen K, Cole PA, Kuriyan J. (2006). An allosteric mechanism for activation of the kinase domain of epidermal growth factor receptor. Cell 125, 1137-49. 